\title{
PSEUDO LIMITS, BI-ADJOINTS, AND PSEUDO ALGEBRAS: CATEGORICAL FOUNDATIONS OF CONFORMAL FIELD THEORY
}

\author{
THOMAS M. FIORE
}

\section{INTRODUCTION}

The purpose of this paper is to work out the categorical basis for the foundations of Conformal Field Theory. The definition of Conformal Field Theory was outlined in Segal [45] and recently given in 24] and 25]. Concepts of 2-category theory, such as versions of algebra, limit, colimit, and adjunction, are necessary for this definition.

The structure present on the class $\mathcal{C}$ of rigged surfaces is captured by these concepts of 2-category theory. Here a rigged surface is a real, compact, not necessarily connected, two dimensional manifold with complex structure and analytically parameterized boundary components. Isomorphisms of such rigged surfaces are holomorphic diffeomorphisms preserving the boundary parameterizations. These rigged surfaces and isomorphisms form a groupoid and are part of the structure present on $\mathcal{C}$. Concepts of 2-categories enter when we describe the operations of disjoint union of two rigged surfaces and gluing of two rigged surfaces along boundary components of opposite orientation. One needs a mathematical structure to capture all of these features. This has been done in [24].

One step in this direction is the notion of algebra over a theory in the sense of Lawvere 34]. We need a weakened notion in which relations are replaced by coherence isos. This weakened notion is called a pseudo algebra in this paper. Coherence diagrams are required in a pseudo algebra, but it was noticed in 24. that Lawvere's notion of a theory allows us to write down all such diagrams easily. See Section [6 below. A symmetric monoidal category as defined in [39] provides us with a classical example of a pseudo algebra over the theory of commutative monoids. Theories, duality, and related topics are discussed further in [1], 2], [3], [35, 36.

Unfortunately, pseudo algebras over a theory are not enough to capture the structure on $\mathcal{C}$. The reason is that the operation of gluing is indexed by the variable set of pairs of boundary components of opposite orientation. The operation of disjoint union also has an indexing. We need pseudo algebras over a "theory indexed over another theory," which we call a 2-theory. More precisely, the pseudo algebras we need are pseudo algebras over the 2-theory of commutative monoids with cancellation. See 24 and Section 12 below. The term 2-theory does not mean a theory in 2-categories.

Date: November 9, 2018. 
Nevertheless, 2-categories are relevant. This is because we want to capture the behavior of holomorphic families of rigged surfaces in our description of the structure of $\mathcal{C}$. This amounts to saying that $\mathcal{C}$ is a stack of pseudo commutative monoids with cancellation. To consider this, we must remark that pseudo algebras over a theory and pseudo algebras over a 2-theory form 2-categories. A stack is a contravariant pseudo functor from a Grothendieck site into a 2-category which takes Grothendieck covers into limits of certain type, which are called bilimits. They are defined below, in [29], and [50, while a slightly stronger notion is called pseudo limit in [50]. One needs to understand such notions for the rigorous foundations of Conformal Field Theory. More elaborate notions, such as analogous kinds of colimits are also needed in 25.

In this article I introduce the general concepts of weighted bilimits, weighted bicolimits, and bi-adjoints for pseudo functors between 2-categories in the sense below and prove statements about their existence in certain cases. There are many versions of such concepts and many (but not all) of the theorems I give are in the literature, see [8, 10, 13, [18, 20], 21], 22], 27], 28, 29], 46], 47, [49, [50, and [51. The circumstances of Conformal Field Theory suggest a particular choice of concepts. To a topologist, the most natural and naive choice of terminology may be to use the term "lax" to mean "up to coherence isos" with these coherence isos required to satisfy appropriate coherence diagrams. "Iso" seems to be the only natural concept in the case of pseudo algebras over a theory: there seems to be no reasonable notion where coherences would not be iso. For this reason, the authors of [24, 25], and [26] use the "lax=up to coherence isos" philosophy. This terminology however turns out to be incorrect from the point of view of category theory (other ad hoc terminology also appears in [24, [25], and [26]). In this paper, I decided to follow established categorical terminology while giving a precise translation of the notions in [24, [25], and [26]. In the established categorical terminology, what is called a lax algebra in [24, 25], and [26] is called a pseudo algebra, what is called a lax morphism (morphism which commutes with operations up to coherence isos) in 24], 25], and [26] is called a pseudo morphism (or just morphism), and what is called a lax functor in 24, 25], and 26] is called a pseudo functor. In addition, the notions which the authors of [24, 25], and 26] refer to as lax limit, lax colimit, and lax adjoint are called bilimit, bicolimit, and bi-adjoint in established categorical terminology. The stronger categorical notions of pseudo limit, pseudo colimit, and pseudo adjoint are also sometimes relevant.

The term "lax" in standard categorical terminology is reserved for notions "up to 2-cells which are not necessarily iso". However, such notions will not play a central role in the present paper, as our motivation here is the same as in [24], 25], and [26], namely Conformal Field Theory and stacks.

I show that every pseudo functor from a 1-category to the 2-category of small categories has both a pseudo limit and a pseudo colimit by constructive proofs. Furthermore, the 2-category of small categories admits weighted pseudo limits and weighted pseudo colimits. After that I introduce the notions of a theory, an algebra over a theory, and a pseudo algebra over a theory. I then go on to show that any pseudo functor from a 1-category to the 2-category of pseudo $T$-algebras has a pseudo limit by using the constructions from the proof of the category case. After a proof of the existence of cotensor products in the 2-category of pseudo $T$-algebras, 
I conclude from a theorem of Street that this 2-category admits all weighted pseudo limits.

I continue the study of weakened structures by turning to bi-adjoints. First I show that a pseudo functor has a left bi-adjoint if and only if for each object of the source category we have an appropriate bi-universal arrow in analogy to the standard result in 1-category theory. By means of this description I show that for any morphism of theories $\phi: S \rightarrow T$ the associated forgetful 2-functor from the 2 -category of pseudo $T$-algebras to the 2-category of pseudo $S$-algebras has a left biadjoint. The formalism developed for bi-adjoints is then adapted to treat bicolimits of pseudo $T$-algebras in the same section. Moreover, the universal property of these bicolimits is slightly weaker than the universal property of the pseudo limits. Similarly, the 2-category of pseudo $T$-algebras admits bitensor products, and hence also weighted bicolimits.

Lastly, I construct pseudo limits of pseudo algebras over a 2-theory. Again, a theorem of Street and existence of cotensor products imply that the 2-category of pseudo algebras over a 2-theory admits all weighted pseudo limits. An example of a pseudo algebra over a 2-theory comes from the category of rigged surfaces in 24].

Some of these results may be found in some form in the literature. There are many different ways to weaken 1-categorical concepts. The following study only sets up the weakened notions needed for utilizing stacks to rigorously define conformal field theory as in 24. Results about bilimits can be found in the references mentioned above. In particular, Gray explicitly describes quasi limits and quasi colimits of strict 2-functors from an arbitrary small 2-category to the 2-category Cat of small categories on pages 201 and 219 of [18, although his quasi limit is defined in terms of quasi adjunction rather than cones. In any case, he does not have formulas for pseudo limits of pseudo functors. Street has the most general result in this context. In [50], he states that Cat admits all indexed pseudo limits of pseudo functors and writes down the indexed pseudolimit. His indexed pseudo limit is the same as the weighted pseudo limit in this paper. Results about notions similar to the notion of bi-adjoint can be found in [18], [19], 29], and [50]. These similarities are discussed in the introduction to the section on bi-adjoints. Blackwell, Kelly, and Power have limit and adjoint results similar to ours for strict 2-functors into 2 -categories of strict algebras and pseudo morphisms over a 2-monad in 9]. In fact, we prove below that pseudo algebras over a theory are the strict algebras for a 2-monad below.

Any discussion of weakened algebraic structures must involve coherence questions. Coherence questions were treated by Laplaza, Mac Lane, and others as early as the 70's. Some recent treatments in the context of $n$-categories and categorification are 4, 5], and [14.

I thank Igor Kriz for his careful guidance, F. W. Lawvere, Ross Street, Steve Lack, John Baez, Tibor Beke, Bob Bruner, James McClure, Jeff Smith, Art Stone, Martin Hyland, John Power, Michael Johnson, Mark Weber, and Bart Kastermans for helpful comments.

I follow the usual convention that 2-categories are denoted by capital script letters $\mathcal{A}, \mathcal{C}, \mathcal{D}, \mathcal{X}$, pseudo functors are denoted by capital letters $F, G$, morphisms are denoted by $e, f, g, h$, and 2 -cells are denoted by Greek letters $\alpha, \beta, \gamma$. The identity 2-cell on a morphism $f$ is denoted $i_{f}$. Natural transformations and pseudo natural transformations are also denoted by lowercase Greek letters. The double 
arrow $\Rightarrow$ is used to denote 2-cells, natural transformations, and pseudo natural transformations, which in some cases are all the same thing. 


\section{Contents}

1. Introduction 1

2. Weighted Pseudo Limits in a 2-Categorv 6

3. Weighted Pseudo Colimits in the 2-Categorv of Small Categories 18

4. Weighted Pseudo Limits in the 2-Categorv of Small Categories 26

5. Theories and Algebras 32

6. Pseudo T-Algebras $\quad 50$

7. Weighted Pseudo Limits in the 2-Categorv of Pseudo T-Algebras 60

8. Bi-universal Arrows and Bi-adioints 67

9. Forgetful 2-Functors For Pseudo Algebras 98

10. Weighted Bicolimits of Pseudo T-algebras 110

$\begin{array}{ll}\text { 11. Stacks } & 117\end{array}$

12. 2-Theories. Algebras. and Weighted Pseudo Limits 125

12.1. The 2-Theorv End(X) Fibered over the Theorv End(I) 125

12.2. 2-Theories and Algebras over 2-Theories 132

12.3. The Algebraic Structure of Rigged Surfaces 133

12.4. Weighted Pseudo Limits of Pseudo $(\Theta . T)$-algebras 136

$\begin{array}{ll}\text { References } & 138\end{array}$ 


\section{Weighted Pseudo Limits in a 2-Category}

In this section I introduce the notion of a weighted pseudo limit and related concepts. The most important examples of 2-categories to keep in mind are the following.

Example 1. The 2-category of small categories is formed by taking the objects (0-cells) to be small categories, the morphisms (1-cells) to be functors, and the 2-cells to be natural transformations. This 2-category is denoted Cat.

Example 2. A full sub-2-category of the previous example is the 2-category with objects groupoids and 1-cells and 2-cells the same as above.

Example 3. An example of a different sort is the 2-category with objects topological spaces, morphisms continuous maps, and 2-cells homotopy classes of homotopies. The 2-cells must be homotopy classes of homotopies in order to make the various compositions associative and unital.

Example 4. Let $\mathcal{J}$ be a small 1-category. Then $\mathcal{J}$ has the the structure of a 2-category if we take $\operatorname{Mor}_{\mathcal{J}}(i, j)$ to be a discrete category for all $i, j \in \operatorname{Obj} \mathcal{J}$.

These examples show that there are two ways of composing the 2-cells: vertically and horizontally. Natural transformations can be composed in two ways. Homotopy classes of homotopies can also be composed in two ways. To clarify which composition I mean, I follow Borceux's notation. See [1] for a more thorough discussion.

Definition 2.1. Let $\mathcal{C}$ be a 2-category. If $A, B \in O b j \mathcal{C}$ and $f, g, h: A \rightarrow B$ are objects of the category $\operatorname{Mor}(A, B)$ with 2-cells $\alpha: f \Rightarrow g$ and $\beta: g \Rightarrow h$ then the composition

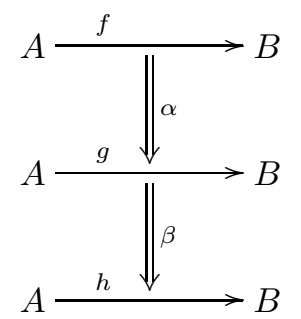

in the category $\operatorname{Mor}(A, B)$ is called the vertical composition of $\alpha$ and $\beta$. The composition is denoted $\beta \odot \alpha$.

Definition 2.2. Let $\mathcal{C}$ be a 2-category and $A, B, C \in \operatorname{ObjC}$. Let $c: \operatorname{Mor}(A, B) \times$ $\operatorname{Mor}(B, C) \rightarrow \operatorname{Mor}(A, C)$ denote the functor of composition in the 2-category $\mathcal{C}$. If $f, g: A \rightarrow B$ and $m, n: B \rightarrow C$ are objects of the respective categories $\operatorname{Mor}(A, B)$ and $\operatorname{Mor}(B, C)$ and $\alpha: f \Rightarrow g, \beta: m \Rightarrow n$ are 2-cells, then the composite 2-cell $c(\alpha, \beta): m \circ f \Rightarrow n \circ g$ is is called the horizontal composition of $\alpha$ and $\beta$. It is a morphism of the category $\operatorname{Mor}(A, C)$ and is denoted $\beta * \alpha$.

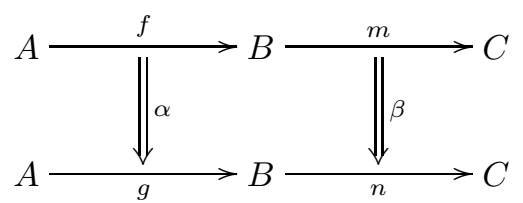


To define the concept of weighted pseudo limit, we need to discuss pseudo functors and pseudo natural transformations. A pseudo functor is like a 2-functor except that it preserves composition and identity only up to iso coherence 2-cells. A pseudo natural transformation is like a 2-natural transformation except that it is natural only up to iso coherence 2 -cell. These coherence 2 -cells must satisfy certain coherences among themselves. We define these notions more carefully to fix some notation. I reproduce Borceux's treatment in 11. The coherence 2-cells for pseudo functors and pseudo natural transformations in this paper are always assumed to be iso. Recall again that a pseudo functor in this paper is a lax functor in [24, 25], and 26] as well as in other previous papers.

Definition 2.3. Let $\mathcal{C}, \mathcal{D}$ be 2-categories. A pseudo functor $F: \mathcal{C} \rightarrow \mathcal{D}$ consists of the following assignments and iso coherence 2-cells:

- For every object $A \in O b j \mathcal{C}$ an object $F A \in O b j \mathcal{D}$

- For every pair of objects $A, B \in O b j \mathcal{C}$ a functor $F: \operatorname{Mor}_{\mathcal{C}}(A, B) \rightarrow$ $\operatorname{Mor}_{\mathcal{D}}(F A, F B)$

- For every triple of objects $A, B, C \in \mathcal{C}$ a natural isomorphism $\gamma$ between the composed functors

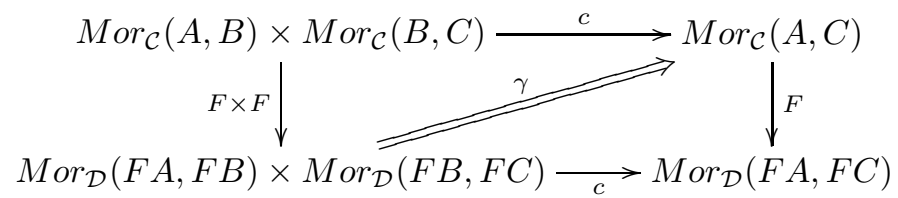

- For every object $A \in \mathcal{C}$ a natural isomorphism $\delta$ between the following composed functors.

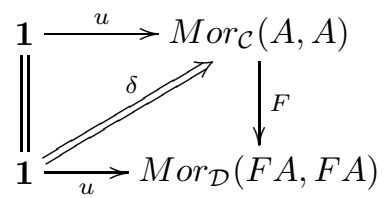

where the functor $u: \mathbf{1} \rightarrow \operatorname{Mor}_{\mathcal{C}}(A, A)$ from the terminal object $\mathbf{1}$ in the category of small categories to the category $\operatorname{Mor}_{\mathcal{C}}(A, A)$ takes the unique object of 1 to the identity morphism on $A$.

These coherence 2-cells must satisfy the following coherence diagrams.

- For every morphism $f: A \rightarrow B$ in $\mathcal{C}$ we require
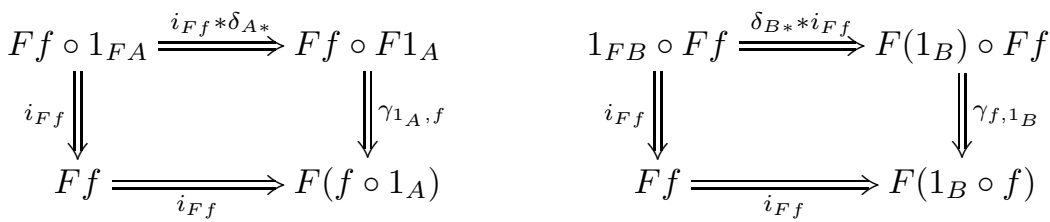

to commute. Here $\delta_{A *}$ means the natural transformation $\delta_{A}$ evaluated at the unique object $*$ of $\mathbf{1}$. This is called the unit axiom for the pseudo functor $F$. 
- For all morphisms $f, g, h$ of $\mathcal{C}$ such that $h \circ g \circ f$ exists we require that

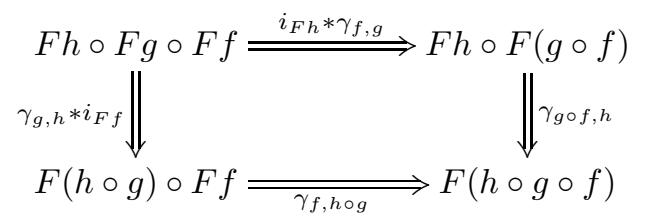

commutes. This is called the composition axiom for the pseudo functor $F$.

Each of these functors and natural transformations of course depends on the objects, so they really need indices, e.g. $c_{A, B, C}, F_{A, B}, \gamma_{A, B, C}, u_{A}, u_{F A}$, and $\delta_{A}$. Often I leave the indices off for more convenient notation. Note that the first diagram in the definition says that the pseudo functor preserves composition of morphisms up to coherence 2-cell because for morphisms $A \stackrel{f}{\longrightarrow} B \stackrel{g}{\longrightarrow} C$ in $\mathcal{C}$ we have $\gamma_{f, g}: F(g) \circ F(f) \Rightarrow F(g \circ f)$ and $\gamma$ is natural in $f$ and $g$. The second diagram in the definition says that the pseudo functor preserves identity up to coherence 2-cell because $\delta_{A *}: 1_{F A} \Rightarrow F\left(1_{A}\right)$ for all $A \in O b j \mathcal{C}$.

Definition 2.4. Let $\mathcal{C} \stackrel{F}{\longrightarrow} \mathcal{D} \stackrel{G}{\longrightarrow} \mathcal{E}$ be pseudo functors. Then the composition $G \circ F$ of pseudo functors is the composition of the underlying maps of objects and the composition of the underlying functors on the morphism categories. The coherence 2-cells are

- For morphisms $A \stackrel{f}{\longrightarrow} B \stackrel{g}{\longrightarrow} C$ in $\mathcal{C}$ the 2-cell $\gamma_{f, g}^{G F}$ is the composition

$$
G F(g) \circ G F(f) \stackrel{\gamma_{F f, F g}^{G}}{=} G(F g \circ F f) \stackrel{G\left(\gamma_{f, g}^{F}\right)}{\Longrightarrow} G F(g \circ f)
$$

- For each object $A \in O b j \mathcal{C}$ the 2-cell $\delta_{A *}^{G F}$ is the composition

$$
1_{G F A} \stackrel{\delta_{F A *}^{G}}{\longrightarrow} G\left(1_{F A}\right) \stackrel{G\left(\delta_{A *}^{F}\right)}{\longrightarrow} G F\left(1_{A}\right)
$$

Then the assignment $(f, g) \mapsto \gamma_{f, g}^{G F}$ is natural and $\gamma^{G F}$ and $\delta_{A}^{G F}$ satisfy the coherences to make $G F$ a pseudo functor.

Definition 2.5. A pseudo natural transformation $\alpha: F \Rightarrow G$ from the pseudo functor $F: \mathcal{C} \rightarrow \mathcal{D}$ to the pseudo functor $G: \mathcal{C} \rightarrow \mathcal{D}$ consists of the following assigments:

- For each $A \in O b j \mathcal{C}$ a morphism $\alpha_{A}: F A \rightarrow G A$ in the category $\mathcal{D}$

- For all objects $A, B \in O b j \mathcal{C}$ a natural isomorphism $\tau$ between the following functors.

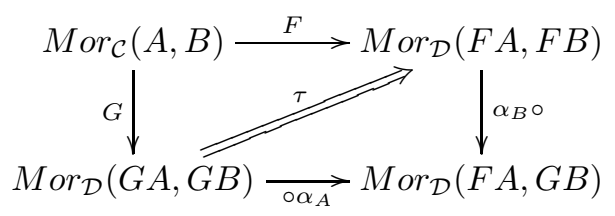

The natural transformations $\tau$ must satisfy the following coherence diagrams involving $\delta$ and $\gamma$. 
- For every $A \in O b j \mathcal{C}$ we require

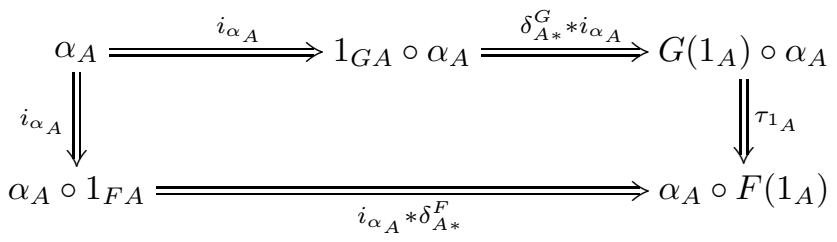

to commute. This is called the unit axiom for the pseudo natural transformation $\alpha$.

- For all morphisms $A \stackrel{f}{\longrightarrow} B \stackrel{g}{\longrightarrow} C$ in $\mathcal{C}$ we require

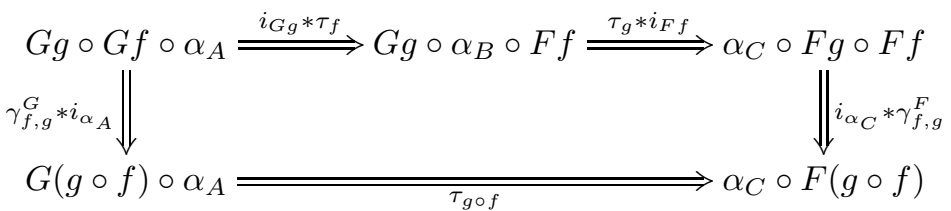

to commute. This is called the composition axiom for the pseudo natural transformation $\alpha$.

Here $\tau$ should of course also be indexed by the objects $A, B$ etc., but we leave off these indices for convenience. The coherence required on $\gamma$ and $\tau$ is the commutativity of the 2-cells (from $\tau$ and $\gamma$ ) written on the faces of the prism with edges $F f, F g, F(g \circ f), G f, G g, G(g \circ f)$ where $f$ and $g$ are composable morphisms in the 2-category $\mathcal{C}$. There are several ways to compose these 2-cells, but they are related by the interchange law. Here one must sometimes horizontally precompose or postcompose a 2-cell with identity 2-cells in order to horizontally compose. Note the diagram for $\tau$ drawn in the definition says that the assignment of $A \mapsto \alpha_{A}$ is natural up to coherence 2-cell because for $f \in \operatorname{Mor}_{\mathcal{C}}(A, B)$ we have the diagram

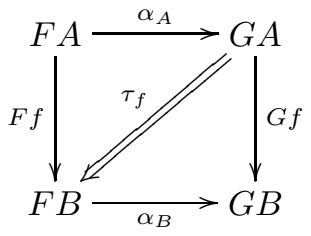

in $\mathcal{D}$. The assignment $f \mapsto \tau_{f}$ is natural in $f$, i.e. $\tau_{A, B}$ is a natural transformation.

Some authors prefer to denote the coherence 2-cells of $\alpha$ by $\alpha_{f}$ instead of $\tau_{f}$. However I follow Borceux's notation in [11 and use the distinguished notation $\tau$ in order to navigate complicated diagrams with less effort.

Pseudo natural transformations can also be horizontally and vertically composed.

For example, if $F \stackrel{\alpha}{\Longrightarrow} G \stackrel{\beta}{\Longrightarrow} H$ are pseudo natural transformations, the vertical composition $\beta \odot \alpha$ has coherence 2-cells $\tau_{f}^{\beta \odot \alpha}=\left(i_{\beta_{B}} * \tau_{f}^{\alpha}\right) \odot\left(\tau_{f}^{\beta} * i_{\alpha_{A}}\right)$ for $f: A \rightarrow B$ as in the following diagram. 


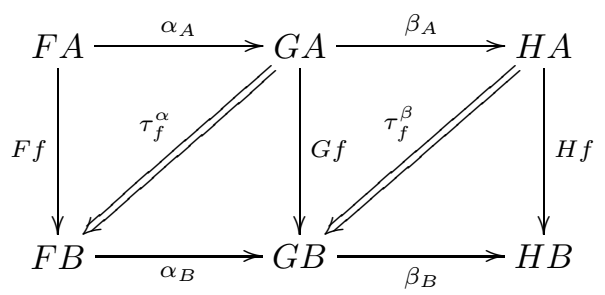

Natural transformations can be seen as morphisms between functors. In the context of 2-categories there is a similar notion of a modification between pseudo natural transformations.

Definition 2.6. Let $F, G: \mathcal{C} \rightarrow \mathcal{D}$ be pseudo functors and $\alpha, \beta: F \Rightarrow G$ pseudo natural transformations. A modification $\Xi: \alpha \rightsquigarrow \beta$ is a function which assigns to every $A \in O b j \mathcal{C}$ a 2 -cell $\Xi_{A}: \alpha_{A} \Rightarrow \beta_{A}$ in $\mathcal{D}$ in such a way that $\tau_{A, B}^{\beta}(g) \odot\left(G \gamma * \Xi_{A}\right)=$ $\left(\Xi_{B} * F \gamma\right) \odot \tau_{A, B}^{\alpha}(f)$ for all $A, B \in O b j \mathcal{C}$ and all morphisms $f, g: A \rightarrow B$ and all 2-cells $\gamma: f \Rightarrow g$. Here $\tau^{\alpha}$ and $\tau^{\beta}$ denote the natural transformations belonging to the pseudo natural transformations $\alpha$ and $\beta$ respectively, while $\gamma$ is an arbitrary 2 -cell in $\mathcal{C}$. This means that the following two compositions of 2-cells are the same.
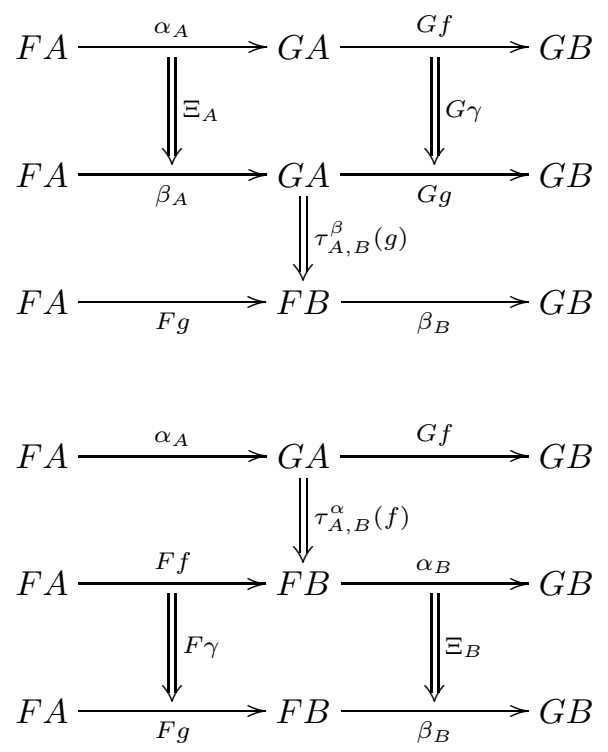

These two diagrams can be combined to make a cube whose faces have 2-cells inscribed in them. In this definition $\gamma$ is not to be confused with the required coherence 2-cell in the definition of pseudo functor.

Definition 2.7. If $F: \mathcal{D} \rightarrow \mathcal{C}$ is a pseudo functor, then a pseudo limit of $F$ consists of an object $W \in O b j \mathcal{C}$ and a pseudo natural transformation $\pi: \Delta_{W} \Rightarrow F$ from the constant 2-functor $W$ to the pseudo functor $F$ which is universal in the following sense: the functor $(\pi \circ): \operatorname{Mor}_{\mathcal{C}}(C, W) \rightarrow$ PseudoCone $(C, F)$ is an isomorphism of categories for every object $C \in O b j \mathcal{C}$. 
PseudoCone $(C, F)$ denotes here the category with objects taken to be the pseudo natural transformations $\Delta_{C} \Rightarrow F$ and with morphisms taken to be the modifications. Pseudo colimits can be defined in terms of PseudoCone $(F, C)$ and $(\circ \pi): \operatorname{Mor}_{\mathcal{C}}(W, C) \rightarrow$ PseudoCone $(F, C)$ similarly.

Theorem 2.1. Any two pseudo limits of a pseudo functor are isomorphic.

Definition 2.8. If $F: \mathcal{D} \rightarrow \mathcal{C}$ is a pseudo functor, then a bilimit of $F$ consists of an object $W \in O b j \mathcal{C}$ and a pseudo natural transformation $\pi: \Delta_{W} \Rightarrow F$ from the constant 2-functor $W$ to the pseudo functor $F$ which is universal in the following sense: the functor $(\pi \circ): \operatorname{Mor}_{\mathcal{C}}(C, W) \rightarrow \operatorname{PseudoCone}(C, F)$ is an equivalence of categories for every object $C \in O b j \mathcal{C}$.

Some authors would call this bilimit a conical bilimit, see [29] and [50 for example. They discuss the more general notion of weighted bilimit or indexed bilimit, which is defined below. Limits defined in terms of cones, such as this bilimit, have constant weight or constant index. For our applications to conformal field theory, it is sufficient to consider only conical bilimits although I prove results for more general weighted bilimits below. The existence of conical bilimits is sufficient to speak of stacks. The term lax limit in 24, 25], and 26] is synonymous with the term bilimit defined above.

Every pseudo limit for a fixed pseudo functor is obviously a bilimit of that pseudo functor. One can ask whether or not bilimits and pseudo limits are the same. The following trivial example shows that bilimits and pseudo limits are not the same.

Example 5. Let 1 denote the terminal object in the category of small categories, in other words $\mathbf{1}$ is the category with one object $*$ and one morphism, namely the identity morphism. This category can be viewed as a 2-category with no nontrivial 2 -cells. Suppose $\mathcal{C}$ is a 2-category with at least two objects $W, W^{\prime}$ such that we have a morphism $\pi^{\prime}: W^{\prime} \rightarrow W$ which is a pseudo isomorphism. This means that there exists a morphism $\theta: W \rightarrow W^{\prime}$ and iso 2-cells $\theta \circ \pi^{\prime} \Rightarrow 1_{W^{\prime}}$ and $\pi^{\prime} \circ \theta \Rightarrow 1_{W}$. Suppose further that $\pi^{\prime}$ is not monic. This means there exists an object $C \in \operatorname{ObjC}$ and distinct morphisms $f_{1}, f_{2}: C \rightarrow W^{\prime}$ such that $\pi^{\prime} \circ f_{1}=\pi^{\prime} \circ f_{2}$. Let $F: \mathbf{1} \rightarrow \mathcal{C}$ be the constant functor $\Delta_{W}$, i.e. $F(*)=W$ and the identity gets mapped to $1_{W}$. Then PseudoCone $(C, F)$ is isomorphic to $\operatorname{Mor}_{\mathcal{C}}(C, W)$. We identify these two categories. Obviously $W$ and the pseudo natural transformation $\pi=1_{W}$ (under the identification) form a pseudo limit, while $W^{\prime}$ and $\pi^{\prime}$ form a bilimit. However, $W^{\prime}$ and $\pi^{\prime}$ do not form a pseudo limit because $\left(\pi^{\prime} \circ\right): \operatorname{Mor}_{\mathcal{C}}\left(C, W^{\prime}\right) \rightarrow \operatorname{Mor}_{\mathcal{C}}(C, W)$ is not an isomorphism of categories, since $\pi^{\prime} \circ f_{1}=\pi^{\prime} \circ f_{2}$ although $f_{1} \neq f_{2}$.

Example 6. There are also examples where a bicolimit exists but not a pseudo colimit. This example goes back to 9]. Let Lex denote the 2-category of small finitely complete categories, left exact functors, and natural transformations. A functor is called left exact if it preserves all finite limits. An initial object is a colimit of the empty 2-functor. A pseudo colimit and a 2-colimit of the empty 2 -functor are the same thing. The 2-category Lex does not admit an initial object because there are always two distinct functors $A \rightarrow I$ where $I$ is the category with only two isomorphic objects and no nontrivial morphisms besides the ismorphism and its inverse. The two constant functors provide us with two distinct functors $A \rightarrow I$ for each $A \in O b j L e x$. The empty functor does however admit a bicolimit because $L e x$ is the 2-category of strict algebras, pseudo algebra morphisms, and 
2-cells for some finitary 2-monad on Cat. Blackwell, Kelly, and Power prove in [9] that such algebra categories admit bicolimits.

Many pseudo algebra categories do not admit pseudo colimits because the morphisms are not strict. Another example can be obtained by adapting Example 19 on page 108 to colimits.

After Example 5, one might wonder whether or not the equivalences of categories in the definition of bilimit can be chosen in some natural way. They can in fact be chosen pseudo naturally as follows. We write it explicitly only for the bicolimit, although a completely analogous statement holds for the bilimit.

Remark 2.2. Let $\mathcal{C}, \mathcal{D}$ be 2-categories. Let $\mathcal{F}: \mathcal{D} \rightarrow \mathcal{C}$ be a pseudo functor. Suppose $W \in O b j \mathcal{C}$ is a bicolimit with universal pseudo cone $\pi: \mathcal{F} \Rightarrow \Delta_{W}$. Let $\phi_{C}$ denote the equivalence of categories $(\circ \pi): \operatorname{Mor}_{\mathcal{C}}(W, C) \rightarrow \operatorname{PseudoCone}(\mathcal{F}, C)$. Let $G(C):=\operatorname{Mor}_{\mathcal{C}}(W, C)$ and $F(C):=\operatorname{PseudoCone}(\mathcal{F}, C)$. Then $G$ and $F$ are strict 2-functors and $C \mapsto \phi_{C}$ is a 2-natural transformation $G \Rightarrow F$.

Proof: This follows from the definitions.

Remark 2.3. Let the notation be the same as in the previous remark. For $C \in$ ObjC let $\psi_{C}: F C \rightarrow G C$ be a right adjoint to $\phi_{C}$ such that the unit $\eta_{C}: 1_{G C} \Rightarrow$ $\psi_{C} \circ \phi_{C}$ and counit $\varepsilon_{C}: \phi_{C} \circ \psi_{C} \Rightarrow 1_{F C}$ are natural isomorphisms. Then $C \mapsto \psi_{C}$ is a pseudo natural transformation from $F$ to $G$ and there exist iso modifications $\eta: i_{G} \rightsquigarrow \psi \odot \phi$ and $\varepsilon: \phi \odot \psi \rightsquigarrow i_{F}$ which satisfy the triangle identities, namely $C \mapsto$ $\eta_{C}$ and $C \mapsto \varepsilon_{C}$. In the terminology of [50, this means that $F$ and $G$ are equivalent in the 2-category Hom $[\mathcal{C}, C a t]$ of pseudo functors, pseudo natural transformations, and modifications. The equivalences in $\mathrm{Hom}[\mathcal{C}, \mathrm{Cat}]$ are precisely the pseudo natural transformations whose components are equivalences of categories.

Proof: $\quad$ Since $\phi_{C}$ is an equivalence of categories, there exists such a functor $\psi_{C}$ with unit and counit as above. For $f: A \rightarrow B$ in $\mathcal{C}$ define the coherence iso $\tau_{f}^{\psi}: G f \circ \psi_{A} \Rightarrow \psi_{B} \circ F f$ to be the composition of 2-cells in the following diagram.

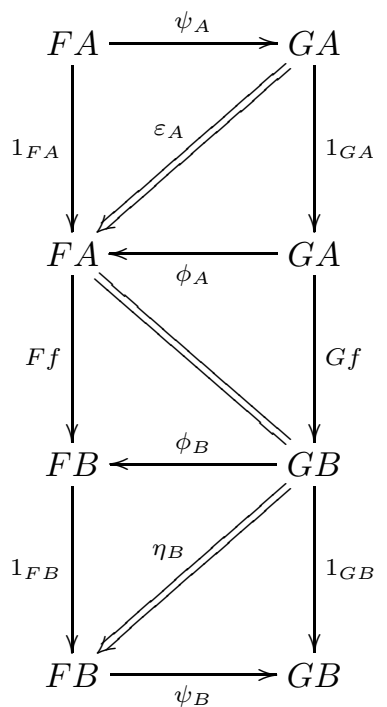


The middle square commutes because $\phi$ is a 2-natural transformation. One can see that the assignment $f \mapsto \tau_{f}^{\psi}$ is natural after segmenting the naturality diagram into three inner squares and using the fact that $\phi$ is a 2-natural transformation as follows. Let $f, g: A \rightarrow B$ and $\mu: f \rightarrow g$ in $\mathcal{C}$.

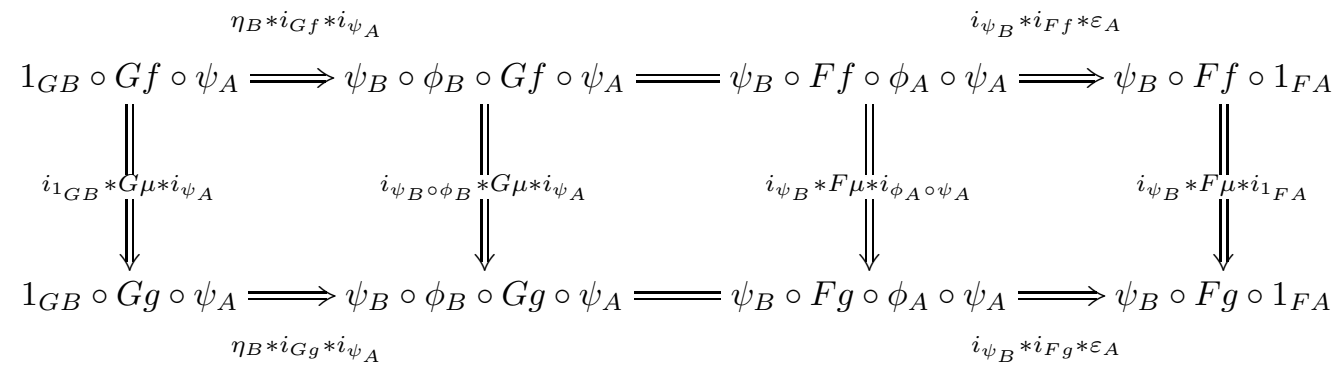

The left square and the right square commute because of the interchange law and the defining property of identity 2-cells. The middle square commutes because $\phi$ is a 2-natural transformation. Hence the outermost rectangle commutes and $f \mapsto \tau_{f}^{\psi}$ is natural.

Since $F$ and $G$ are strict 2-functors, verifying the unit axiom for $\psi$ reduces to proving that $\tau_{1_{C}}^{\psi}$ is $i_{\psi_{C}}$ for all $C \in O b j \mathcal{C}$. That follows from the definition of $\tau_{1_{C}}$ and one of the triangle identities.

Since $F$ and $G$ are strict 2-functors, verifying the composition axiom for $\psi$ amounts to proving for $A \stackrel{f}{\longrightarrow} B \stackrel{g}{\longrightarrow} C$ in $\mathcal{C}$ that the composition $\left(\tau_{g}^{\psi} * i_{F f}\right) \odot$ 
$\left(i_{G g} * \tau_{f}^{\psi}\right)$ in (3) is the same as $\tau_{g \circ f}^{\psi}$ in (4). That follows since the middle parallelogram in (4) is $i_{\phi_{B}}$ by the triangle identity. Hence $\psi$ with $\tau^{\psi}$ satisfies the composition axiom and we conclude that $C \mapsto \psi_{C}$ is a pseudo natural transformation $F \Rightarrow G$.

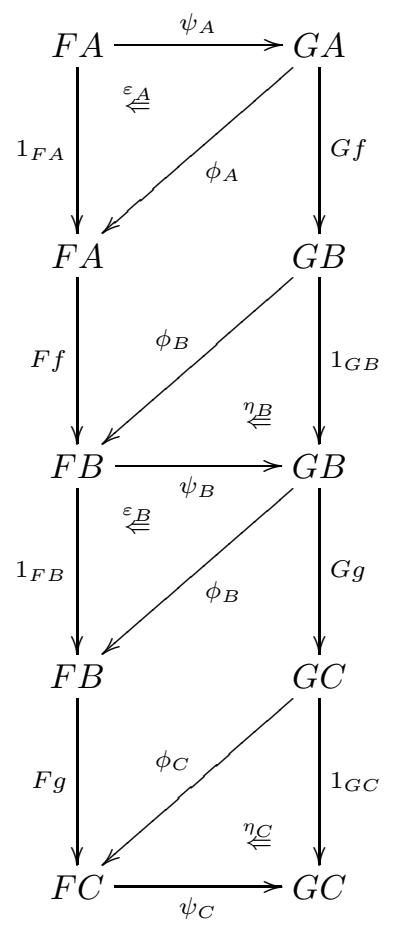

(4)

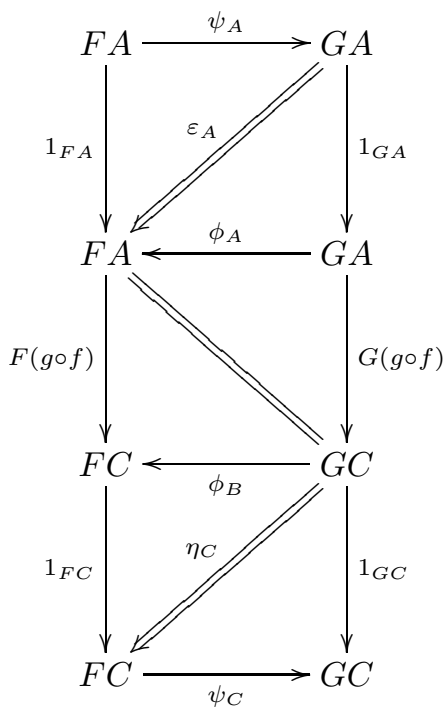

Next I prove that $A \mapsto \eta_{A}$ is a modification $i_{G} \rightsquigarrow \psi \odot \phi$. This entails showing that (11) is the same as (2). Let $f, g: A \rightarrow B$ be morphisms in $\mathcal{C}$ and $\gamma: f \Rightarrow g$ a 2-cell in $\mathcal{C}$. Since $\phi$ is a 2-natural transformation, we see that (2) is $\eta_{B} * G \gamma$. 
I proceed by showing that (10) is $\eta_{B} * G \gamma$. Note that $\tau_{A, B}^{\beta}(g)=\tau_{g}^{\psi \circ \phi}$ in (1) is $\left(i_{\psi_{B}} * i_{\phi_{B} \circ G g}\right) \odot\left(\tau_{g}^{\psi} * i_{\phi_{A}}\right)$ by the remarks on page 9 about coherence isos for a vertical composition of pseudo natural transformations. Writing out (1) with $\alpha=i_{G}$,

$\beta=\psi \odot \phi, \Xi=\eta$, and including many trivial arrows gives (5).

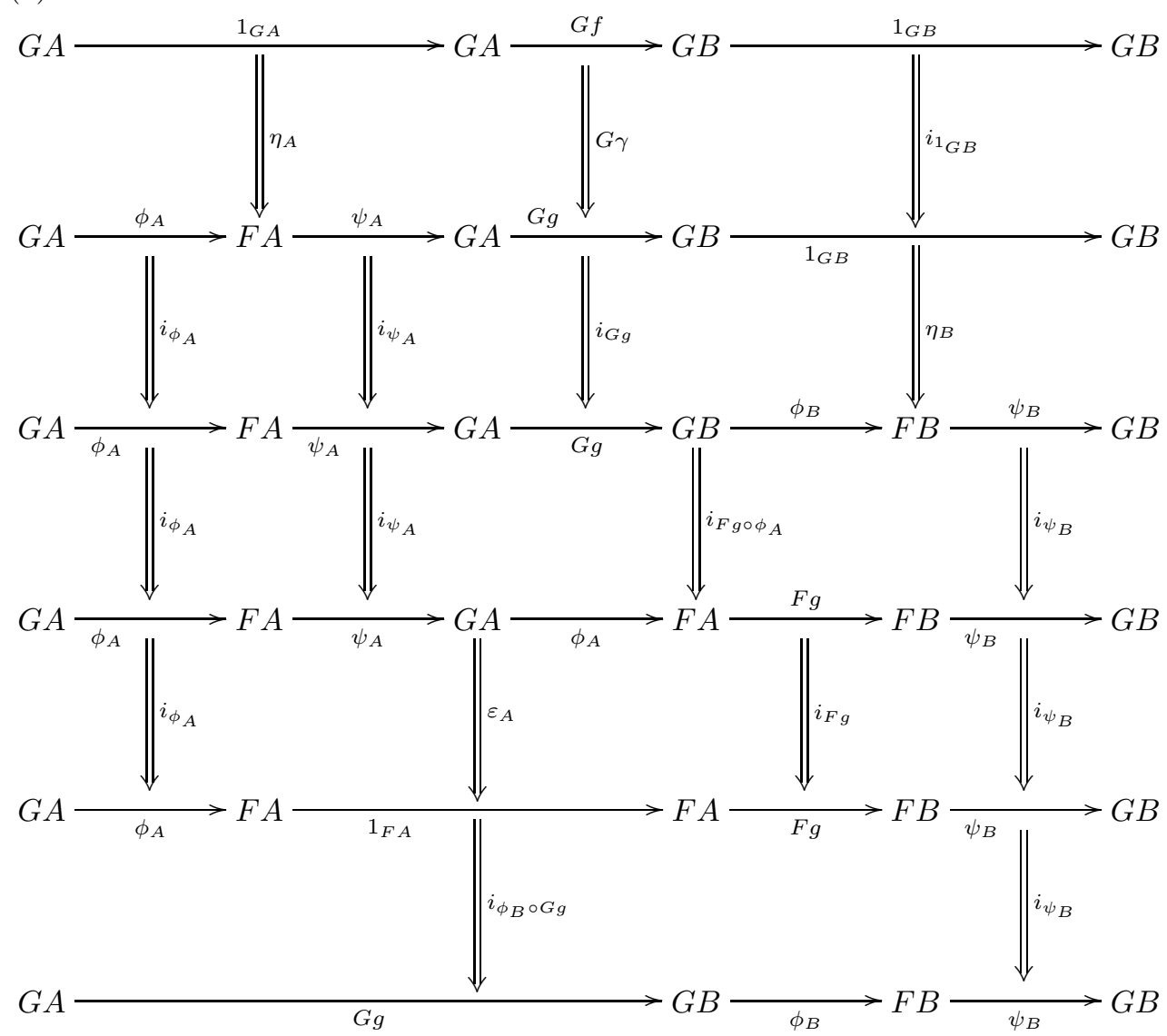

Using a triangle identity and contracting all the trivial identities, we see that the only thing that does not cancel is $\eta_{B} * G \gamma$. Hence (1) is the same as (2) and $A \mapsto \eta_{A}$ is a modification.

One can similarly show that $A \mapsto \varepsilon_{A}$ is a modification. The modifications $\eta$ and $\varepsilon$ satisfy the triangle identities because their constituent arrows do.

Definition 2.9. A 2-category $\mathcal{C}$ admits bilimits if every pseudo functor $F: \mathcal{J} \rightarrow \mathcal{C}$ from a small 1-category $\mathcal{J}$ to $\mathcal{C}$ has a bilimit in $\mathcal{C}$.

In this definition we are of course viewing the category $\mathcal{J}$ as a 2-category with no nontrivial 2-cells. There are analogous definitions for bicolimits and pseudo colimits. If we view the category $\mathcal{J}$ as an indexing category, then we can speak of bilimits of diagrams, i.e. we can view a diagram in $\mathcal{C}$ as the image of a pseudo functor from a source diagram $\mathcal{J}$ to the 2-category $\mathcal{C}$. 
The concept of pseudo limit can be further generalized to weighted pseudo limit. For any small 2-category $\mathcal{C}$ we denote the small category $H_{\mathcal{C}}(A, B)$ by $\mathcal{C}(A, B)$ for $A, B \in O b j \mathcal{C}$.

Definition 2.10. Let $\mathcal{C}, \mathcal{D}$ be 2-categories. Let $J: \mathcal{D} \rightarrow C a t$ and $F: \mathcal{D} \rightarrow \mathcal{C}$ be pseudo functors. Let $\operatorname{Hom}[\mathcal{D}, \mathrm{Cat}]$ denote the 2-category with pseudo functors $\mathcal{D} \rightarrow$ Cat as objects, pseudo natural transformations as morphisms, and modifications as 2-cells. Then $\{J, F\}_{p} \in O b j \mathcal{C}$ is called a $J$-weighted pseudo limit of $F$ if the strict 2-functors $\mathcal{C}^{o p} \rightarrow$ Cat

$$
\begin{gathered}
C \mapsto \mathcal{C}\left(C,\{J, F\}_{p}\right) \\
C \mapsto \operatorname{Hom}[\mathcal{D}, C a t](J, \mathcal{C}(C, F-))
\end{gathered}
$$

are 2-isomorphic. The image $\xi: J \Rightarrow \mathcal{C}\left(\{J, F\}_{p}, F-\right)$ of $1_{\{J, F\}_{p}}$ under this 2representation is called the unit.

Street refers to this as the $J$-indexed pseudo limit of $F$ in [50], although now the term weighted is used instead of indexed. This is similar to Kelly's definition in 29, except that his definition is for strict 2-functors $J, F$ and he uses the full sub-2-category $P s d[\mathcal{D}, C a t]$ of $\operatorname{Hom}[\mathcal{D}, C a t]$ in place of $H o m[\mathcal{D}, C a t]$. The 2category $P s d[\mathcal{D}, C a t]$ consists of strict 2 -functors, pseudo natural transformations, and modifications.

We recover the usual definition of pseudo limit whenever $J$ is the constant functor which takes everything to the trivial category with one object. A weighted pseudo limit is said to be conical whenever $J$ is the constant functor just mentioned. Another special type of weighted limit called cotensor product occurs when $\mathcal{D}$ is the trivial 2-category with one object and $J$ and $F$ are strict 2 -functors. In this case $J$ and $F$ can be identified with objects of $C a t$ and $\mathcal{C}$ respectively. Tensor products can be defined similarly.

Definition 2.11. Let $J \in O b j C a t$ and $F \in O b j \mathcal{C}$. Then $\{J, F\} \in O b j \mathcal{C}$ is called a cotensor product of $J$ and $F$ if the strict 2-functors $\mathcal{C}^{o p} \rightarrow C a t$

$$
\begin{gathered}
C \mapsto \mathcal{C}(C,\{J, F\}) \\
C \mapsto \operatorname{Cat}(J, \mathcal{C}(C, F))
\end{gathered}
$$

are 2-naturally isomorphic.

Remark 2.1. (Kelly) We can rephrase the definition of cotensor product entirely in terms of the unit $\pi: J \rightarrow \mathcal{C}(\{J, F\}, F)$. The object $\{J, F\}$ of $\mathcal{C}$ is a cotensor product of $J$ and $F$ with unit $\pi: J \rightarrow \mathcal{C}(\{J, F\}, F)$ if and only if the functor $\mathcal{C}(C,\{J, F\}) \rightarrow \operatorname{Cat}(J, \mathcal{C}(C, F))$ defined by composition with $\pi$

$$
\begin{aligned}
b & \mapsto \mathcal{C}(b, F) \circ \pi \\
\alpha & \mapsto \mathcal{C}(\alpha, F) * i_{\pi}
\end{aligned}
$$

for arrows $b: C \rightarrow\{J, F\}$ and 2-cells $\alpha: b \rightarrow b^{\prime}$ in $\mathcal{C}$ is an isomorphism of categories for all $C \in O b j \mathcal{C}$. More specifically,

(1) For every functor $\sigma: J \rightarrow \mathcal{C}(C, F)$ there is a unique arrow $b: C \rightarrow\{J, F\}$ in $\mathcal{C}$ such that $\mathcal{C}(b, F) \circ \pi=\sigma$

(2) For every natural transformation $\Xi: \sigma \Rightarrow \sigma^{\prime}$ there is a unique 2-cell $\alpha: b \Rightarrow b^{\prime}$ in $\mathcal{C}$ such that $\mathcal{C}(\alpha, F) * i_{\pi}=\Xi$. 
A useful reformulation of an observation by Street on page 120 of [50] illustrates the importance of cotensor products in the context of weighted pseudo limits.

Theorem 2.4. (Street) A 2-category $\mathcal{C}$ admits all weighted pseudo limits if and only if it admits 2-products, cotensor products, and pseudo equalizers.

Remark 2.2. (Street) Pseudo equalizers can be constructed from cotensor products and 2-pullbacks, while 2-pullbacks can be constructed from 2-products and 2 -equalizers. Thus it is sufficient to require 2-equalizers instead of pseudo equalizers in the previous theorem.

Definition 2.12. Let $\mathcal{C}, \mathcal{D}$ be 2-categories. Let $J: \mathcal{D} \rightarrow C$ at and $F: \mathcal{D} \rightarrow \mathcal{C}$ be pseudo functors. As above, let $\operatorname{Hom}[\mathcal{D}, \mathrm{Cat}]$ denote the 2-category with pseudo functors $\mathcal{D} \rightarrow C a t$ as objects, pseudo natural transformations as morphisms, and modifications as 2-cells. Then $\{J, F\}_{b} \in O b j \mathcal{C}$ is called a $J$-weighted bilimit of $F$ if the strict 2-functors $\mathcal{C}^{o p} \rightarrow$ Cat

$$
\begin{gathered}
C \mapsto \mathcal{C}\left(C,\{J, F\}_{b}\right) \\
C \mapsto \operatorname{Hom}[\mathcal{D}, C a t](J, \mathcal{C}(C, F-))
\end{gathered}
$$

are equivalent in the 2-category $\operatorname{Hom}\left[\mathcal{C}^{o p}, C a t\right]$, i.e. there is a pseudo natural transformation going from one to the other whose arrow components are equivalences of categories. The image $\xi: J \Rightarrow \mathcal{C}\left(\{J, F\}_{b}, F-\right)$ of $1_{\{J, F\}_{b}}$ under this birepresentation is called the unit.

Kelly refers to this in [29] as the $J$-indexed bilimit of $F$. The concepts weighted bicolimit and bitensor product can be defined similarly. Later we will need bitensor products, so we formulate this precisely and describe it entirely in terms of the unit like Kelly in 29].

Definition 2.13. Let $J \in O b j C a t$ and $F \in O b j \mathcal{C}$. Then $J * F \in O b j \mathcal{C}$ is called a bitensor product of $J$ and $F$ if the strict 2-functors $\mathcal{C}^{o p} \rightarrow C a t$

$$
\begin{gathered}
C \mapsto \mathcal{C}(J * F, C) \\
C \mapsto \operatorname{Cat}(J, \mathcal{C}(F, C))
\end{gathered}
$$

are are equivalent in the 2-category $\operatorname{Hom}\left[\mathcal{C}^{o p}, C a t\right]$.

Remark 2.3. We can rephrase the definition of bitensor product entirely in terms of the unit $\pi: J \rightarrow \mathcal{C}(F, J * F)$. The object $J * F$ of $\mathcal{C}$ is a bitensor product of $J$ and $F$ with unit $\pi: J \rightarrow \mathcal{C}(F, J * F)$ if and only if the functor $\mathcal{C}(J * F, C) \rightarrow \operatorname{Cat}(J, \mathcal{C}(F, C))$ defined by

$$
\begin{aligned}
b & \mapsto \mathcal{C}(F, b) \circ \pi \\
\alpha & \mapsto \mathcal{C}(F, \alpha) * i_{\pi}
\end{aligned}
$$

for arrows $b: J * F \rightarrow C$ and 2-cells $\alpha: b \rightarrow b^{\prime}$ in $\mathcal{C}$ is an equivalence of categories for all $C \in O b j \mathcal{C}$.

Street points out the dual version of the following theorem on page 120 of [50].

Theorem 2.5. (Street) A 2-category $\mathcal{C}$ admits all weighted bicolimits if and only if it admits bicoproducts, bitensor products, and bicoequalizers.

Cotensor products, bitensor products, and the theorems above will be used later to show that the 2-categories of interest to us admit weighted pseudo limits as well as weighted bicolimits. 


\section{Weighted Pseudo Colimits in the 2-Category of Small Categories}

In this section I show constructively that the 2-category $\mathcal{C}$ of small categories admits pseudo colimits. A theorem of Street will imply that this 2-category also admits weighted pseudo colimits. One of the concepts in the proof is the free category generated by a directed graph.

Definition 3.1. A directed graph $G$ consists of a set $O$ of objects and a set $A$ of arrows and two functions $S, T: A \rightarrow O$ called source and target.

A directed graph is like a category except composition and identity arrows are not necessarily defined. Any directed graph $G$ whose sets of arrows and objects are both small generates a free category on $G$, which is also called the path category of $G$. Similarly $G$ generates a free groupoid. One can force commutivity of certain diagrams by putting an equivalence relation on the morphism sets of the free category or free groupoid and then passing to the quotient category. I use this construction in the proof below. The $S, T$ in the definition of directed graph will also be used to denote the source and target of a morphism in a category, although they are different concepts.

Theorem 3.1. The 2-category $\mathcal{C}$ of small categories admits pseudo colimits.

Proof: $\quad$ Let $\mathcal{J}$ be a small 1-category and $F: \mathcal{J} \rightarrow \mathcal{C}$ a pseudo functor. Here we view $\mathcal{J}$ as a 2 -category which has no nontrivial 2-cells. The category $\mathcal{J}$ plays the role of an indexing category. For any $X \in O b j \mathcal{C}$ let $\Delta_{X}$ denote the constant 2functor which takes every object of $\mathcal{J}$ to $X$, every morphism to $1_{X}$, and every 2-cell to the identity 2-cell $i_{X}: 1_{X} \Rightarrow 1_{X}$. Then a pseudo cone from $F$ to $X$ is a pseudo natural transformation $F \Rightarrow \Delta_{X}$. Let $P \operatorname{seudoCone}(F, X)$ denote the category with objects the pseudo cones from $F$ to $X$ with morphisms the modifications between them. The pseudo colimit of $F$ is an object $W \in \mathcal{C}$ with a pseudo cone $\pi: F \Rightarrow \Delta_{W}$ which are universal in the sense that $(\circ \pi): \operatorname{Mor}_{\mathcal{C}}(W, V) \rightarrow P \operatorname{PseudoCone}(F, V)$ is an isomorphism of categories for all small categories $V$.

First I define candidates $W \in O b j \mathcal{C}$ and $\pi: F \Rightarrow \Delta_{W}$. Then I show that they are universal. For each $j \in O b j \mathcal{J}$ let $A_{j}$ denote the small category $F j$ and let $a_{f}$ denote the functor $F f$ between small categories. Since $F$ is a pseudo functor, for every pair $f, g$ of morphisms of $\mathcal{J}$ such that $g \circ f$ exists we have a natural transformation (a 2-cell in the 2-category of small categories) $\gamma_{f, g}: F g \circ F f \Rightarrow F(g \circ f)$. I define a directed graph with objects $O$ and arrows $A$ as follows. Let $O=\coprod_{j \in \mathcal{J}} O b j A_{j}$. There is a well defined function $p: O \rightarrow O b j \mathcal{J}$ satisfying $p\left(O b j A_{j}\right)=\{j\}$ because this union is disjoint, i.e. even if the small categories $A_{i}$ and $A_{j}$ are the same, we distinguish them in the disjoint union by their indices. Let the collection of arrows be $A=\left(\coprod_{j \in \mathcal{J}} \operatorname{Mor} A_{j}\right) \amalg\left\{h_{(x, f)}, h_{(x, f)}^{-1}:(x, f) \in O \times M o r \mathcal{J}\right.$ such that $p(x)=S f\}$ where the elements of $\coprod_{j \in \mathcal{J}}$ Mor $A_{j}$ have the obvious source and target while $S h_{(x, f)}=x$ and $T h_{(x, f)}=a_{f}(x)$. Let $W^{\prime}$ be the free category generated by this graph. We put the smallest equivalence relation $\sim$ on $M$ or $W^{\prime}$ such that

- All of the relations in each $A_{i}$ are contained in $\sim$, i.e. for $m, n \in$ Mor $A_{i} \subseteq$ $M o r W^{\prime}$ with $S n=T m$ we have $n \circ_{W^{\prime}} m \sim n \circ_{A_{i}} m$ where the composition on the left is the composition in the free category $W^{\prime}$ and the composition on the right is the composition in the small category $A_{i}$. 
- For all $f, g \in M o r \mathcal{J}$ with $S g=T f$ and all $x \in O b j A_{S f}$ we have $\gamma_{f, g}(x) \circ_{W^{\prime}} h_{\left(a_{f}(x), g\right)}{ }^{\circ} W^{\prime} h_{(x, f)} \sim h_{(x, g \circ f)}$ and also every identity $1_{x} \in A_{i}$ is equivalent to the identity in the free category on the object $x$.

- For all $i, j \in O b j \mathcal{J}$ and all $f \in \operatorname{Mor}_{\mathcal{J}}(i, j)$ and all morphisms $m: x \rightarrow y$ of $A_{i}$ we have $h_{(y, f)} \circ_{W^{\prime}} m \sim a_{f}(m) \circ_{W^{\prime}} h_{(x, f)}$.

- For all $j \in O b j \mathcal{J}$ and all $x \in O \operatorname{Obj} A_{j}$ we have $\left(\delta_{j *}^{F}\right)_{x} \sim h_{\left(x, 1_{j}\right)}$ where * denotes the unique object of the terminal object 1 in the category of small categories and $\delta_{j *}^{F}$ is the natural transformation $\delta_{j}^{F}$ evaluated at $*$.

- For all $h_{(x, f)}$ from above we have $h_{(x, f)}^{-1}{ }^{\circ} W^{\prime} h_{(x, f)} \sim 1_{x}$ and $h_{(x, f)}{ }^{\circ} W^{\prime}$ $h_{(x, f)}^{-1} \sim 1_{a_{f} x}$

Define $W$ to be the quotient category of the free category $W^{\prime}$ by the equivalence relation $\sim$. This is the candidate for the pseudo colimit.

Now I define a pseudo natural transformation $\pi: F \Rightarrow \Delta_{W}$ and its coherence 2-cells $\tau$, i.e. I define an element of $P \operatorname{seudoCone}(F, W)$. For each object $j \in \operatorname{Obj} \mathcal{J}$ we need a morphism in $\mathcal{C}$ (i.e. a functor) $\pi_{j}: F j=A_{j} \rightarrow W=\Delta_{W}(j)$. Define $\pi_{j}: A_{j} \rightarrow W$ to be the inclusion functors $A_{j} \hookrightarrow W$. In order for $\pi$ to be a pseudo natural transformation, this assignment must be natural up to coherence 2-cell, i.e. for all $i, j \in O b j \mathcal{J}$ we should have a natural isomorphism $\tau_{i, j}$ of the following sort.

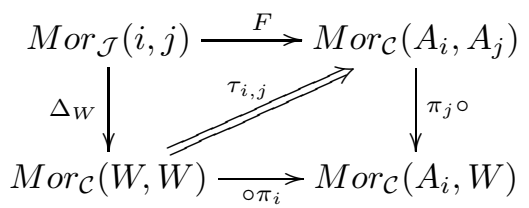

Evaluating this diagram at a morphism $f: i \rightarrow j$ of $\mathcal{J}$ we should have a natural isomorphism between functors $\tau_{i, j}(f): \pi_{i} \Rightarrow \pi_{j} \circ a_{f}$. In other words, $\tau_{i, j}(f)$ should be a 2 -cell in the 2-category $\mathcal{C}$ of small categories. For each $x \in \operatorname{Obj} A_{i}$ define $\tau_{i, j}(f)_{x}: \pi_{i}(x)=x \rightarrow a_{f}(x)=\pi_{j} \circ a_{f}(x)$ to be the isomorphism $h_{(x, f)}$.

Lemma 3.2. The map $\pi: F \Rightarrow \Delta_{W}$ is a pseudo natural transformation with coherence 2-cells given by the natural isomorphisms $\tau$.

Proof: I continue the notation from the comments before the Lemma. First I show that the assignment $\operatorname{Obj}_{i} \ni x \mapsto \tau_{i, j}(f)_{x} \in \operatorname{Mor}_{W}\left(\pi_{i}(x), \pi_{j} \circ a_{f}(x)\right)$ is a natural transformation for fixed $f: i \rightarrow j$. To this end, let $m: x \rightarrow y$ be a morphism in the small category $A_{i}$. By definition, $\tau_{i, j}(f)_{x}=h_{(x, f)}, \tau_{i, j}(f)_{y}=$ $h_{(y, f)}, \pi_{i}(m)=m, \pi_{i}(x)=x, \pi_{j} \circ a_{f}(x)=a_{f}(x)$, and $\pi_{j} \circ a_{f}(m)=a_{f}(m)$. Some similar statements hold for the object $y$. The third requirement on the equivalence relation in $W^{\prime}$ gives us the following commutative diagram in the small category $W$.

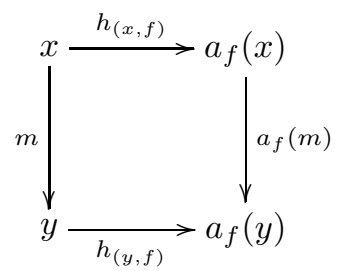

Using the identities just mentioned, the commutivity of this diagram says precisely that $x \mapsto \tau_{i, j}(f)_{x}$ is a natural transformation. Thus $\tau_{i, j}(f): \pi_{i} \Rightarrow \pi_{j} \circ a_{f}$ is a 
natural transformation between functors, i.e. a 2-cell in the 2-category $\mathcal{C}$ of small categories.

The assignment $f \mapsto \tau_{i, j}(f)$ for fixed $i, j$ is natural because the category $\operatorname{Mor}_{\mathcal{J}}(i, j)$ has no nontrivial morphisms. Thus $\tau_{i, j}$ is a natural transformation between the indicated functors.

Next I verify the composition axiom for pseudo natural transformations which involves $\tau$ and $\gamma$. The diagram states that $\tau$ must satisfy for all $i \stackrel{f}{\longrightarrow} j \stackrel{g}{\longrightarrow} k$ in $\mathcal{J}$ the coherence axiom $\left(i_{\pi_{k}} * \gamma_{f, g}\right) \odot\left(\tau_{j, k}(g) * i_{a_{f}}\right) \odot\left(i_{1_{W}} * \tau_{i, j}(f)\right)=\tau_{i, k}(g \circ$ $f) \odot\left(i_{1_{W}} * i_{\pi_{i}}\right)$ as natural transformations. This coherence is satisfied because of the second requirement on the relation in $W^{\prime}$ for each $x \in \operatorname{Obj} A_{i}$ which states $\gamma_{f, g}(x) \circ \tau_{j, k}(g)_{a_{f}(x)} \circ \tau_{i, j}(f)_{x}=\tau_{i, k}(g \circ f)_{x}$. Note that $\left(i_{\pi_{k}} * \gamma_{f, g}\right)(x)=\pi_{k}\left(\gamma_{f, g}(x)\right)=$ $\gamma_{f, g}(x)$.

Lastly I verify the unit axiom for pseudo natural transformations which involves $\tau$ and $\delta$. This coherence requires the commutivity of the following diagram for all $j \in O b j \mathcal{J}$.

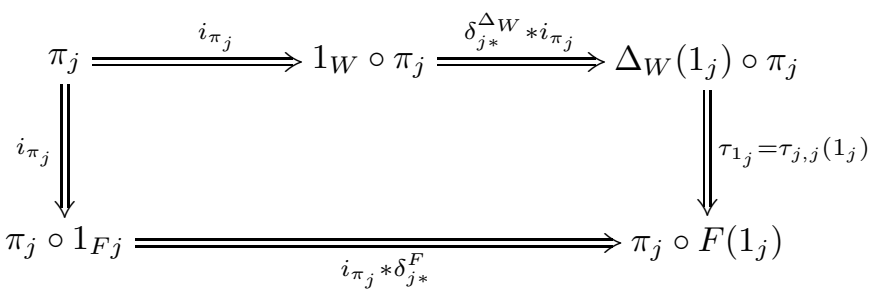

Here $\delta_{j}^{\Delta_{W}}$ and $\delta_{j}^{F}$ are the natural transformations associated to the pseudo functors $\Delta_{W}$ and $F$ which make them preserve the identity morphisms $1_{j}$ up to coherence 2-cell. In fact, $\delta_{j *}^{\Delta_{W}}$ is trivial. If $\mathbf{1}$ denotes the terminal object of the category of small categories, then $\delta_{j}^{\Delta_{W}}$ and $\delta_{j}^{F}$ must satisfy the following diagrams for all objects $j$ of $\mathcal{J}$.
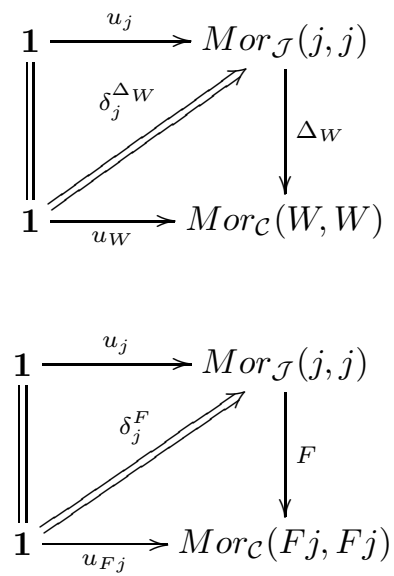

Using the fact that $\delta_{j}^{\Delta_{W}}$ evaluated on the unique object $*$ of $\mathbf{1}$ gives the identity 2-cell $i_{W}: 1_{W} \Rightarrow 1_{W}$, the desired coherence diagram simplifies to the following. 


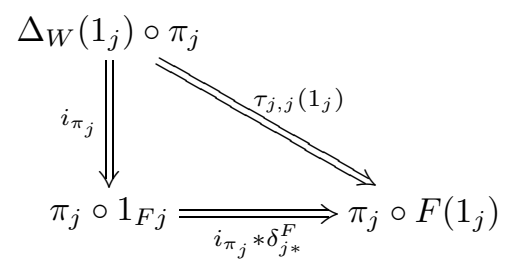

Recall that $\left(\delta_{j *}^{F}\right)_{x}=h_{\left(x, 1_{j}\right)}$ in $W$ by the fourth requirement on the equivalence relation in $W^{\prime}$. By definition we also have $h_{\left(x, 1_{j}\right)}=\tau_{j, j}\left(1_{j}\right)_{x}$. This implies $\left(\delta_{j *}^{F}\right)_{x}=h_{\left(x, 1_{j}\right)}=\tau_{j, j}\left(1_{j}\right)_{x}$ and the simplified diagram commutes because $\pi_{j}$ is the inclusion functor. Hence the required coherence diagram involving $\tau$ and $\delta$ is actually satisfied.

Thus $\pi: F \Rightarrow \Delta_{W}$ is a pseudo natural transformation with the indicated coherence 2-cells.

Now I must show that the small category $W$ and the pseudo natural transformation $\pi: F \Rightarrow \Delta_{W}$ are universal in the sense that the functor $\phi: \operatorname{Mor}_{\mathcal{C}}(W, V) \rightarrow$ PseudoCone $(F, V)$ defined by $\phi(b)=b \circ \pi$ for objects $b$ is an isomorphism of categories for all objects $V$ of $\mathcal{C}$. More precisely, $\phi$ is defined for $b \in \operatorname{ObjMor}_{\mathcal{C}}(W, V)$ and $j \in \operatorname{Obj} \mathcal{J}$ as $\phi(b)(j)=b \circ \pi_{j}$. The coherence 2-cells for the pseudo cone $\phi(b)$ are $i_{b} * \tau_{i, j}(f)$ for all $f: i \rightarrow j$ in $\mathcal{J}$. For morphisms $\gamma: b \Rightarrow b^{\prime}$ in $\operatorname{MorMor}_{C}(W, V)$ I define $\phi(\gamma): b \circ \pi \rightsquigarrow b^{\prime} \circ \pi$ to be the modification which takes $j \in \operatorname{Obj} \mathcal{J}$ to $\phi(\gamma)(j)=\gamma * i_{\pi_{j}}$. Here $i_{\pi_{j}}$ denotes as usual the identity 2-cell $\pi_{j} \Rightarrow \pi_{j}$ between these morphisms of $\mathcal{C}$. In the following, $V$ is a fixed object of the 2-category $\mathcal{C}$ of small categories.

Lemma 3.3. The map $\phi: \operatorname{Mor}_{\mathcal{C}}(W, V) \rightarrow$ PseudoCone $(F, V)$ is a functor.

Proof: $\quad$ Let $b \in \operatorname{ObjMor}_{\mathcal{C}}(W, V)$ be a functor and $i_{b}: b \Rightarrow b$ its identity natural transformation. Then obviously $\phi\left(i_{b}\right)(j)=i_{b} * i_{\pi_{j}}: b \circ \pi_{j} \Rightarrow b \circ \pi_{j}$ is the identity natural transformation $i_{b \circ \pi_{j}}$ for all $j \in O b j \mathcal{J}$ and thus $\phi\left(i_{b}\right)$ is the identity modification. Hence $\phi$ preserves identities.

To verify that $\phi$ preserves compositions, let $\gamma: b \Rightarrow b^{\prime}$ and $\gamma^{\prime}: b^{\prime} \Rightarrow b^{\prime \prime}$ be natural transformations. Then for each $j \in O b j \mathcal{J}$ we have $\phi\left(\gamma^{\prime} \odot \gamma\right)(j)=\left(\gamma^{\prime} \odot \gamma\right) * i_{\pi_{j}}=$ $\left(\gamma^{\prime} \odot \gamma\right) *\left(i_{\pi_{j}} \odot i_{\pi_{j}}\right)$. By the interchange law we have $\left(\gamma^{\prime} \odot \gamma\right) *\left(i_{\pi_{j}} \odot i_{\pi_{j}}\right)=$ $\left(\gamma^{\prime} * i_{\pi_{j}}\right) \odot\left(\gamma * i_{\pi_{j}}\right)=\left(\phi\left(\gamma^{\prime}\right)(j)\right) \odot(\phi(\gamma)(j))=\left(\phi\left(\gamma^{\prime}\right) \diamond \phi(\gamma)\right)_{j}$ where the last equality follows from the definition of vertical composition of modifications. Thus $\phi\left(\gamma^{\prime} \odot \gamma\right)=\phi\left(\gamma^{\prime}\right) \diamond \phi(\gamma)$ and $\phi$ preserves compositions. Thus $\phi$ is a functor.

The purpose of the next few lemmas is to exhibit an inverse functor $\psi$ for $\phi$.

Lemma 3.4. There is a functor $\psi:$ PseudoCone $(F, V) \rightarrow \operatorname{Mor}_{\mathcal{C}}(W, V)$.

Proof: $\quad$ First I define $\psi$ for objects. Then I define $\psi$ for morphisms. Finally I verify that $\psi$ is a functor.

Let $\pi^{\prime}$ be an object of PseudoCone $(F, V)$, i.e. $\pi^{\prime}: F \Rightarrow \Delta_{V}$ is a pseudo natural transformation with coherence 2-cells $\tau^{\prime}$ up to which $\pi^{\prime}$ is natural. To define a functor $\psi \pi^{\prime}=b \in \operatorname{ObjMor}_{\mathcal{C}}(W, V)$ I use the universal mapping property of the quotient category $W$ as follows. Define an auxiliary functor $d: W^{\prime} \rightarrow V$ as the functor induced by the map of directed graphs below which is also called $d$. 
- For all $i \in O b j \mathcal{J}$ and $x \in O b j A_{i} \subseteq O b j W^{\prime}$ let $d x:=\pi_{i}^{\prime} x$

- For all $i \in O b j \mathcal{J}, x, y \in O b j A_{i}$, and all $g \in \operatorname{Mor}_{A_{i}}(x, y) \subseteq \operatorname{Mor}_{W^{\prime}}(x, y)$ let $d g:=\pi_{i}^{\prime} g$

- For all $i, j \in O b j \mathcal{J}, f \in M o r_{\mathcal{J}}(i, j)$, and all $x \in O b j A_{i} \subseteq O b j W^{\prime}$ define $d\left(h_{(x, f)}\right):=\tau_{i, j}^{\prime}(f)_{x}: \pi_{i}^{\prime} x \rightarrow \pi_{j}^{\prime} \circ a_{f} x$ and $d\left(h_{(x, f)}^{-1}\right):=\tau_{i, j}^{\prime}(f)_{x}^{-1}: \pi_{j}^{\prime} \circ a_{f} x \rightarrow \pi_{i}^{\prime} x$.

I claim that $d$ preserves the equivalence relation placed on the category $W^{\prime}$. Following the order in the definition of $\sim$ we have the verifications

- For $m, n \in \operatorname{Mor} A_{i} \subseteq M$ Mor $W^{\prime}$ with $S n=T m$ we have $d\left(n \circ_{W^{\prime}} m\right)=$ $d n \circ_{V} d m=\pi_{i} n \circ_{V} \pi_{i} m=\pi_{i}\left(n \circ_{A_{i}} m\right)=d\left(n \circ_{A_{i}} m\right)$ and for all $1_{x} \in A_{i}$ we have $d 1_{x}=\pi_{i}^{\prime}\left(1_{x}\right)=1_{\pi_{i}^{\prime} x}$ because $\pi_{i}^{\prime}$ is a functor. But $1_{\pi_{i}^{\prime} x}$ is also the same as $d$ applied to the identity on $x$ in the free category $W^{\prime}$.

- Since $\pi^{\prime}$ is a pseudo natural transformation, for all $i \stackrel{f}{\longrightarrow} j \stackrel{g}{\longrightarrow} k$ in $\mathcal{J}$ we have

$\left(i_{\pi_{k}^{\prime}} * \gamma_{f, g}\right) \odot\left(\tau_{j, k}^{\prime}(g) * i_{a_{f}}\right) \odot\left(i_{1_{V}} * \tau_{i, j}^{\prime}(f)\right)=\tau_{i, k}^{\prime}(g \circ f) \odot\left(i_{1_{V}} * i_{\pi_{i}}\right)$ as natural transformations. Evaluating this at $x \in O \operatorname{Obj} A_{i}$ yields $\left(\pi_{k}^{\prime} \gamma_{f, g}(x)\right) \circ \tau_{j, k}^{\prime}(g)_{a_{f} x} \circ \tau_{i, j}^{\prime}(f)_{x}=\tau_{i, k}^{\prime}(g \circ f)_{x}$. This says precisely $d\left(\gamma_{f, g}(x) \circ_{W^{\prime}} h_{\left(a_{f}(x), g\right)} \circ_{W^{\prime}} h_{(x, f)}\right)=d\left(h_{(x, g \circ f)}\right)$.

- For all $i, j \in O b j \mathcal{J}$ and all $f \in \operatorname{Mor}_{\mathcal{J}}(i, j)$ and all morphisms $m: x \rightarrow y$ of $A_{i}$ we have to show $d\left(h_{(y, f)} \circ_{W^{\prime}} m\right)=d\left(a_{f}(m){ }^{\circ}{ }_{W^{\prime}} h_{(x, f)}\right)$. Writing out $d$, we see that this is the same as verifying $\tau_{i, j}^{\prime}(f)_{y} \circ_{V} \pi_{i}^{\prime} m=\left(\pi_{j}^{\prime} \circ a_{f}\right) m \circ_{V}$ $\tau_{i, j}^{\prime}(f)_{x}$, which is true because the assignment $x \mapsto \tau_{i, j}^{\prime}(f)_{x}$ is a natural transformation from $\pi_{i}^{\prime}$ to $\pi_{j}^{\prime} \circ a_{f}$.

- For all $j \in O b j \mathcal{J}$ and all $x \in O b j A_{j}$ we have to show $d\left(\delta_{j *}^{F}\right)_{x}=d h_{\left(x, 1_{j}\right)}$ where $*$ denotes the unique object of the terminal object 1 in the category of small categories and $\delta_{j *}^{F}$ is the natural transformation $\delta_{j}^{F}$ evaluated at $*$. Writing out $d$ we see that this is the same as verifying $\pi_{j}^{\prime}\left(\delta_{j *}^{F}\right)_{x}=\tau_{j, j}^{\prime}\left(1_{j}\right)_{x}$. Since $\pi^{\prime}$ is a pseudo natural transformation from $F$ to $\Delta_{V}$, the natural transformation $\tau^{\prime}$ must satisfy the coherence $\left(i_{\pi_{j}^{\prime}} * \delta_{j *}^{F}\right) \odot i_{\pi_{j}^{\prime}}=\tau_{j, j}^{\prime}\left(1_{j}\right) \odot$ $\left(i_{1_{V}} * i_{\pi_{j}^{\prime}}\right) \odot i_{\pi_{j}^{\prime}}$ as natural transformations. Evaluating this coherence at $x \in O b j A_{j}$ we get $\pi_{j}^{\prime}\left(\delta_{j *}^{F}\right)_{x} \circ 1_{\pi_{j}^{\prime} x}=\tau_{j, j}^{\prime}\left(1_{j}\right)_{x} \circ 1_{\pi_{j}^{\prime} x} \circ 1_{\pi_{j}^{\prime} x}$, which implies $d\left(\delta_{j *}^{F}\right)_{x}=d h_{\left(x, 1_{j}\right)}$ by the remarks above.

- For all $i, j \in \operatorname{Obj} \mathcal{J}, f \in \operatorname{Mor}_{\mathcal{J}}(i, j)$, and all $x \in O b j A_{i} \subseteq O b j W^{\prime}$ we have $d\left(h_{(x, f)}^{-1}{ }^{\circ} W^{\prime} h_{(x, f)}\right)=\tau_{i, j}^{\prime}(f)_{x}^{-1} \circ \tau_{i, j}^{\prime}(f)_{x}=1_{\pi_{j}^{\prime} x}=d\left(1_{x}\right)$ and similarly $d\left(h_{(x, f)}{ }^{\circ} W^{\prime} h_{(x, f)}^{-1}\right)=d\left(1_{a_{f} x}\right)$.

Thus $d: W^{\prime} \rightarrow V$ is a functor that preserves the equivalence relation on $W^{\prime}$. By the universal mapping property of quotient category $W$ of $W^{\prime}$, there exists a unique functor $b: W \rightarrow V$ which factors $d$ via the projection. Define $\psi\left(\pi^{\prime}\right):=$ $b \in \operatorname{ObjMor}_{\mathcal{C}}(W, V)$. This is how $\psi$ is defined on the objects of the category PseudoCone $(F, V)$.

Next I define $\psi$ on morphisms of the category PseudoCone $(F, V)$. Let $\Xi$ : $\sigma \rightsquigarrow \sigma^{\prime}$ be a morphism of PseudoCone $(F, V)$, i.e. $\Xi$ is a modification from the pseudo natural transformation $\sigma: F \Rightarrow \Delta_{V}$ to the pseudo natural transformation $\sigma^{\prime}: F \Rightarrow \Delta_{V}$. Let $\tau$ and $\tau^{\prime}$ respectively denote the natural transformations that make the pseudo natural transformations $\sigma$ and $\sigma^{\prime}$ natural up to cell. I define a morphism $\psi(\Xi)$ of $\operatorname{Mor}_{\mathcal{C}}(W, V)$ as follows. Note that such a morphism is by 
definition a natural transformation between functors from the small category $W$ to the small category $V$. Since $\Xi$ is a modification, we have a 2-cell $\Xi_{i}: \sigma_{i} \Rightarrow \sigma_{i}^{\prime}$ in the category $\mathcal{C}$ for each $i \in O b j \mathcal{J}$. Let $b, b^{\prime}$ denote the respective functors $\psi(\sigma), \psi\left(\sigma^{\prime}\right)$ : $W \rightarrow V$. For $x \in \operatorname{Obj}_{i} \subseteq O \operatorname{Obj} W$ define $\psi(\Xi)_{x}: b x=\sigma_{i} x \rightarrow \sigma_{i}^{\prime} x=b^{\prime} x$ to be $\Xi_{i}(x): \sigma_{i} x \rightarrow \sigma_{i}^{\prime} x$. The following two commutative diagrams show that $\psi(\Xi)$ is a natural transformation. For $x, y \in \operatorname{Obj}_{i}$ and $m \in \operatorname{Mor}_{A_{i}}(x, y) \subseteq \operatorname{Mor}_{W}(x, y)$ the diagram

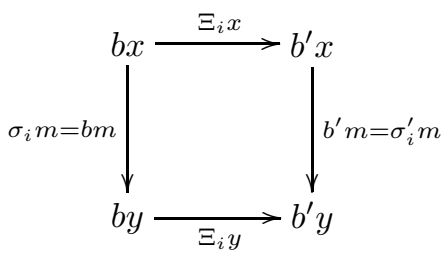

in $V$ commutes because $\Xi_{i}: \sigma_{i} \Rightarrow \sigma_{i}^{\prime}$ is a natural transformation. For a morphism $f: i \rightarrow j$ of $\mathcal{J}$ the diagram

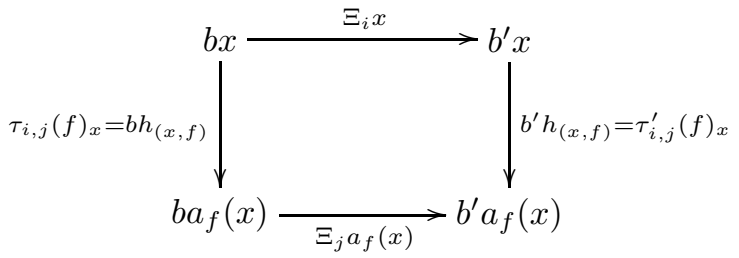

commutes because of the coherence in the definition of modification and because of the definitions of $b, b^{\prime}$ on $h_{(x, f)}$. One sees this by taking $\gamma=i_{f}$ in diagrams (11) and (2) in the definition of modification. An inductive argument shows that $\psi(\Xi)$ is natural for all other arrows in $W$ as well. Hence $\psi(\Xi): \psi(\sigma) \Rightarrow \psi\left(\sigma^{\prime}\right)$ is a morphism of the category $\operatorname{Mor}_{\mathcal{C}}(W, V)$.

Lastly I verify that $\psi$ is a functor, i.e. that $\psi$ preserves the identity modifications and the composition of modifications. Let $\Xi: \sigma \rightsquigarrow \sigma$ be the identity modification belonging to a pseudo natural transformation $\sigma: F \Rightarrow \Delta_{V}$. This means that $\Xi_{i}: \sigma_{i} \Rightarrow \sigma_{i}$ is the identity natural transformation for the functor $\sigma_{i}: A_{i} \rightarrow V$. For all $i \in \operatorname{Obj} \mathcal{J}$ and all $x \in \operatorname{Obj} A_{i}$ we have by definition of $\psi$ that $\psi(\Xi)_{x}: \psi(\sigma) x=$ $\sigma_{i} x \rightarrow \sigma_{i} x=\psi(\sigma) x$ is $\Xi_{i}(x): \sigma_{i} x \rightarrow \sigma_{i} x$, which is the identity morphism on the object $\sigma_{i} x$ of the small category $V$ by hypothesis. Hence $\psi(\Xi): \psi(\sigma) \rightarrow \psi(\sigma)$ is the identity natural transformation and $\psi$ preserves identity modifications.

To verify that $\psi$ preserves compositions, let $\Xi: \sigma \rightsquigarrow \sigma^{\prime}$ and $\Xi^{\prime}: \sigma^{\prime} \rightsquigarrow \sigma^{\prime \prime}$ be modifications. Then the vertical composition of modifications (which makes PseudoCone $(F, V)$ a category) is defined as $\left(\Xi^{\prime} \diamond \Xi\right)_{i}:=\Xi_{i}^{\prime} \odot \Xi_{i}$ where $\Xi_{i}^{\prime} \odot \Xi_{i}$ is the vertical composition of the natural transformations $\Xi_{i}: \sigma_{i} \Rightarrow \sigma_{i}^{\prime}$ and $\Xi_{i}^{\prime}: \sigma_{i}^{\prime} \Rightarrow \sigma_{i}^{\prime \prime}$ as usual. Then for all $i \in \operatorname{Obj} \mathcal{J}$ and all $x \in \operatorname{Obj} A_{i} \subseteq \operatorname{Obj} W$ we have $\psi\left(\Xi^{\prime} \diamond \Xi\right)_{x}=$ $\left(\Xi^{\prime} \diamond \Xi\right)_{i}(x)=\left(\Xi_{i}^{\prime} \odot \Xi_{i}\right)_{x}=\Xi_{i}^{\prime}(x) \circ \Xi_{i}(x)=\psi\left(\Xi^{\prime}\right)_{x} \circ \psi(\Xi)_{x}=\left(\psi\left(\Xi^{\prime}\right) \odot \psi(\Xi)\right)_{x}$. Thus $\psi\left(\Xi^{\prime} \diamond \Xi\right)=\psi\left(\Xi^{\prime}\right) \odot \psi(\Xi)$ and $\psi$ preserves compositions of modifications. Hence $\psi$ is a functor.

Lemma 3.5. The composite functor $\phi \circ \psi: P \operatorname{seudoCone}(F, V) \rightarrow P \operatorname{seudoCone}(F, V)$ is the identity functor. 
Proof: $\quad$ First I verify this for objects, then on for morphisms. Let $\pi^{\prime}: F \Rightarrow \Delta_{V}$ be a pseudo natural transformation with coherence isomorphisms $\tau^{\prime}$. Let $b=\psi\left(\pi^{\prime}\right)$. Then using the definitions of $b$ in Lemma 3.4 and the definition of $\pi$ above we evaluate $\phi\left(\psi\left(\pi^{\prime}\right)\right)$ at each object $i$ of $\mathcal{J}$ and compare the resulting functor $\phi\left(\psi\left(\pi^{\prime}\right)\right)_{i}$ to the functor $\pi_{i}^{\prime}$. Formally this is:

- For all $x \in \operatorname{Obj} A_{i}$, we have $\phi\left(\psi\left(\pi^{\prime}\right)\right)_{i} x=\phi(b)_{i} x=\left(b \circ \pi_{i}\right) x=b x=\pi_{i}^{\prime} x$

- For all $x, y \in \operatorname{Obj} A_{i}$ and all $g \in \operatorname{Mor}_{A_{i}}(x, y)$ we have $\phi\left(\psi\left(\pi^{\prime}\right)\right)_{i} g=\phi(b)_{i} g=\left(b \circ \pi_{i}\right) g=b g=\pi_{i}^{\prime} g$.

Thus $\phi\left(\psi\left(\pi^{\prime}\right)\right)=\pi^{\prime}$ for all objects $\pi^{\prime}$ of the category PseudoCone $(F, V)$. Hence $\phi \circ \psi$ is the identity on objects.

Next I verify the lemma for morphisms. Let $\Xi: \sigma \rightsquigarrow \sigma^{\prime}$ be a morphism of the category PseudoCone $(F, V)$, i.e. $\Xi$ is a modification from the pseudo natural transformation $\sigma: F \Rightarrow \Delta_{V}$ to the pseudo natural transformation $\sigma^{\prime}: F \Rightarrow \Delta_{V}$. Let $b=\psi(\sigma), b^{\prime}=\psi\left(\sigma^{\prime}\right): W \rightarrow V$ and $\gamma=\psi(\Xi): b \Rightarrow b^{\prime}$ for more convenient notation. Then $\phi(\psi(\Xi))=\phi(\gamma): b \circ \pi \rightsquigarrow b^{\prime} \circ \pi$ is a modification from $\sigma$ to $\sigma^{\prime}$ by the result on objects. For each $j \in O b j \mathcal{J}$ we have the natural transformation $\phi(\gamma)(j)=\gamma * i_{\pi_{j}}: b \circ \pi_{j} \Rightarrow b^{\prime} \circ \pi_{j}$. But this natural transformation is precisely $\Xi_{j}: \sigma_{j} \Rightarrow \sigma_{j}^{\prime}$ by the definition of $\gamma$ via $\psi$. Thus for all morphisms $\Xi$ of the category PseudoCone $(F, V)$ we have $\phi(\psi(\Xi))=\Xi$. Hence $\phi \circ \psi$ is the identity on morphisms.

Lemma 3.6. The composite functor $\psi \circ \phi: \operatorname{Mor}_{\mathcal{C}}(W, V) \rightarrow \operatorname{Mor}_{\mathcal{C}}(W, V)$ is the identity functor.

Proof: First I verify this for objects, then on generators for morphisms. Let

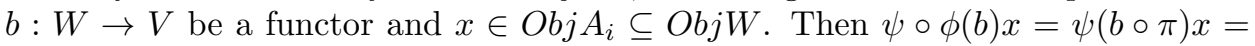
$\left(b \circ \pi_{i}\right) x=b x$. Similarly for a morphism $g \in \operatorname{Mor}_{A_{i}}(x, y) \subseteq \operatorname{Mor}_{W}(x, y)$ we have $\psi \circ \phi(b) g=\psi(b \circ \pi) g=\left(b \circ \pi_{i}\right) g=b g$. For morphisms $h_{(x, f)}$, the analogous calculation is $\psi \circ \phi(b) h_{(x, f)}=\psi(b \circ \pi) h_{(x, f)}=\left(i_{b} * \tau_{i, j}(f)\right)_{x}=b\left(\tau_{i, j}(f)_{x}\right)=b h_{(x, f)}$. That follows because the coherence 2-cell up to which $b \circ \pi$ is natural is $\left(i_{b} * \tau_{i, j}(f)\right)_{x}=b\left(\tau_{i, j}(f)_{x}\right)$, then we use the third part of the definition of $\psi$ as well as the definition $h_{(x, f)}=\tau_{i, j}(f)_{x}$. Thus $\psi \circ \phi(b)=b$ for all objects $b$ of the category $\operatorname{Mor}_{\mathcal{C}}(W, V)$. Hence $\psi \circ \phi$ is the identity on the objects of the category $\operatorname{Mor}_{\mathcal{C}}(W, V)$.

Next I verify the lemma for morphisms. Let $\gamma: b \Rightarrow b^{\prime}$ be a morphism of $\operatorname{Mor}_{\mathcal{C}}(W, V)$, i.e. a natural transformation from some functor $b$ to some functor $b^{\prime}$. Let $\Xi=\phi(\gamma), \sigma=\phi(b)$, and $\sigma^{\prime}=\phi\left(b^{\prime}\right)$ for more convenient notation. Then by definition $\Xi: \sigma=b \circ \pi \rightsquigarrow b^{\prime} \circ \pi=\sigma^{\prime}$ is the modification which takes $j \in \mathcal{J}$ to $\gamma * i_{\pi_{j}}$. Let $x \in O \operatorname{Obj} A_{j} \subseteq \operatorname{ObjW}$. Then $\psi(\Xi)_{x}: \psi(\sigma) x=\sigma_{j} x \rightarrow \sigma_{j}^{\prime} x=\psi\left(\sigma^{\prime}\right) x$ is $\Xi_{j}(x)=\left(\gamma * i_{\pi_{j}}\right)_{x}:(b \circ \pi)_{j} x \rightarrow\left(b^{\prime} \circ \pi\right)_{j} x$. This is described by the following diagram.

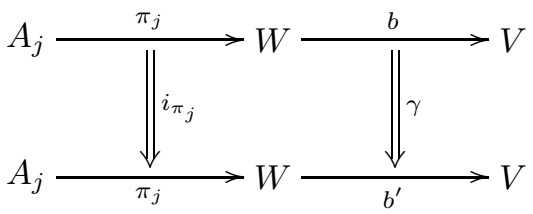


But by definition of $\phi$ and $(b \circ \pi)_{j}$, we see that $\Xi_{j}(x)=\gamma_{\pi_{j} x}=\gamma_{x}$ is precisely $\gamma_{x}: b x \rightarrow b^{\prime} x$. Thus $\psi(\Xi)_{x}=\gamma_{x}$ and $\psi(\phi(\gamma))=\psi(\Xi)=\gamma$. Hence $\psi \circ \phi$ is the identity on the morphisms of the category $\operatorname{Mor}_{\mathcal{C}}(W, V)$.

Lemma 3.7. The small category $W$ and the pseudo natural transformation $\pi: F \Rightarrow$ $\Delta_{W}$ are universal in the sense that the functor $\phi: \operatorname{Mor}_{\mathcal{C}}(W, V) \rightarrow P \operatorname{PseudoCone}(F, V)$ defined by $\phi b=b \circ \pi$ for objects $b$ is an isomorphism of categories for all objects $V$ of $\mathcal{C}$.

Proof: This follows immediately from the previous four lemmas because $V$ was an arbitrary object of the 2-category $\mathcal{C}$.

Lemma 3.8. The small category $W$ and the pseudo natural transformation $\pi: F \Rightarrow \Delta_{W}$ are a pseudo colimit of the pseudo functor $F: \mathcal{J} \rightarrow \mathcal{C}$.

Proof: This follows from Lemma 3.2 and the previous lemma.

Thus every pseudo functor $F: \mathcal{J} \rightarrow \mathcal{C}$ from a small 1-category $\mathcal{J}$ to the 2 category $\mathcal{C}$ of small categories has a pseudo colimit. In other words, the 2 -category $\mathcal{C}$ of small categories admits pseudo colimits. This completes the proof of Theorem 3.1

Lemma 3.9. The 2-category of $\mathcal{C}$ of small categories admits tensor products.

Proof: Let $J$ and $F$ be small categories. Then $J * F:=J \times F$ is a tensor product of $J$ and $F$ with unit $\pi: J \rightarrow C a t(F, J \times F)$ defined by

$$
\begin{aligned}
\pi(j)(x) & :=(j, x) \\
\pi(j)(f) & :=\left(1_{j}, f\right) \\
\pi(g)_{x} & :=\left(g, 1_{x}\right)
\end{aligned}
$$

for $j \in \operatorname{ObjJ}, x \in \operatorname{ObjF}, f \in M o r F, g \in M o r J$. Alternatively one can see that $\operatorname{Cat}(J \times F, C)$ is isomorphic to $\operatorname{Cat}(J, \operatorname{Cat}(F, C))$ by the usual adjunction.

Lemma 3.10. The 2-category $\mathcal{C}$ of small categories admits all weighted pseudo colimits.

Proof: This 2-category admits pseudo coequalizers by Theorem 3.1 It also admits tensor products by Lemma 3.9 It is not difficult to construct 2-coproducts in this 2 -category by using disjoint union. Hence, by the dual version of Theorem 2.4 $\mathcal{C}$ admits all weighted pseudo limits.

Remark 3.11. The 2-category of small groupoids admits weighted pseudo colimits.

Proof: The proof is the same as in the proof for the 2-category of small categories except that one replaces the free category by the free goupoid.

Theorem 3.12. The 2-category of small categories and the 2-category of small groupoids admit weighted bicolimits.

Proof: These 2-categories admit weighted pseudo colimits. Every weighted pseudo colimit is a weighted bicolimit. 


\section{Weighted Pseudo Limits in the 2-Category of Small Categories}

Not only does the 2-category $\mathcal{C}$ of small categories admits pseudo colimits, but it also admits pseudo limits. In fact I construct them explicitly in the next proof. The notation remains the same as in the previous section. This description is not new, since the candidate $L$ in the proof below can be found in [50]. The same construction works for weighted pseudo limits in this 2-category. A theorem from Street also allows us to conclude that $\mathcal{C}$ admits all weighted pseudo limits.

Theorem 4.1. The 2-category $\mathcal{C}$ of small categories admits pseudo limits.

Proof: $\quad$ Let $\mathcal{J}$ be a small 1-category and $F: \mathcal{J} \rightarrow \mathcal{C}$ a pseudo functor, i.e. $\mathcal{J}$ has no nontrivial 2-cells. For any $X \in O b j \mathcal{C}$ let $\Delta_{X}$ denote the constant 2-functor which takes every object of $\mathcal{J}$ to $X$, every morphism to $1_{X}$, and every 2-cell to the identity 2-cell $i_{X}: 1_{X} \Rightarrow 1_{X}$. Then a pseudo cone from $X$ to $F$ is a pseudo natural transformation $\Delta_{X} \Rightarrow F$. Let PseudoCone $(X, F)$ denote the category with objects the pseudo cones from $X$ to $F$ with morphisms the modifications between them. A pseudo limit of $F$ is an object $L \in O b j \mathcal{C}$ with a pseudo cone $\pi: \Delta_{L} \Rightarrow F$ which are universal in the sense that $(\pi \circ): \operatorname{Mor}_{\mathcal{C}}(V, L) \rightarrow P$ seudoCone $(V, F)$ is an isomorphism of categories for all small categories $V$.

First I define candidates $L \in O b j \mathcal{C}$ and $\pi: \Delta_{L} \Rightarrow F$. Then I show that they are universal. For each $j \in O b j \mathcal{J}$ let $A_{j}$ denote the small category $F j$ as in the proof for the pseudo colimit. Let $\mathbf{1}$ denote the small category with one object $*$ and no nontrivial morphisms. Then the candidate for the pseudo limit is $L:=$ PseudoCone $(\mathbf{1}, F)$, also called the category of pseudo cones to $F$ on a point. The pseudo natural transformation candidate $\pi: \Delta_{L} \Rightarrow F$ is defined for all objects $\eta: \Delta_{1} \Rightarrow F$ of $L$ as $\pi_{i}(\eta):=\eta_{i}(*)$ for all $i \in O b j \mathcal{J}$. For morphisms $\Theta: \eta \rightsquigarrow \eta^{\prime}$ of $L$ define $\pi_{i}(\Theta):=\Theta_{i}(*): \eta_{i}(*) \rightarrow \eta_{i}^{\prime}(*)$ for all $i \in \operatorname{Obj} \mathcal{J}$. Define the coherence isos $\tau_{i, j}$

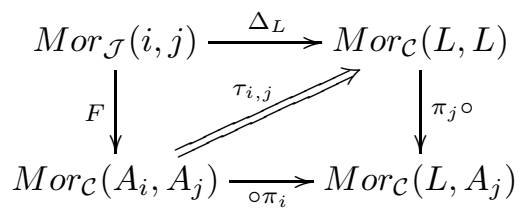

belonging to $\pi: \Delta_{L} \Rightarrow F$ by $\tau_{i, j}(f)_{\eta}:=\tau_{i, j}^{\eta}(f)_{*}$ for all $f \in \operatorname{Mor}_{\mathcal{J}}(i, j)$ and all $\eta \in \operatorname{Obj} L$ where $\tau_{i, j}^{\eta}$ is the coherence natural isomorphism belonging to $\eta: \Delta_{\mathbf{1}} \Rightarrow F$.

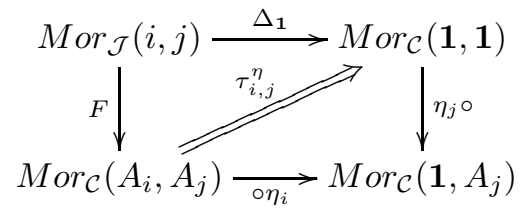

Lemma 4.2. The map $\pi: \Delta_{L} \Rightarrow F$ is a pseudo natural transformation with coherence 2-cells given by $\tau$.

Proof: $\quad$ First I show that for each $j \in O b j \mathcal{J}$ we have a morphism $\pi_{j}: L=$ $\Delta_{L}(j) \rightarrow F j=A_{j}$ in the 2-category $\mathcal{C}$. I claim that $\pi_{j}$ is a morphism, i.e. a functor. Let $1_{\eta}=\Theta: \eta \rightsquigarrow \eta$ be the identity modification of the pseudo cone $\eta: \Delta_{1} \Rightarrow F$. This means $\Theta_{j}=i_{\eta_{j}}: \eta_{j} \Rightarrow \eta_{j}$ is the identity natural transformation for all $j \in \operatorname{Obj} \mathcal{J}$. Then $\pi_{j}\left(1_{\eta}\right)=\pi_{j}(\Theta)=\Theta_{j}(*)=1_{\eta_{j}(*)}=1_{\pi_{j}(\eta)}$ and $\pi_{j}$ preserves 
identities. Now let $\Theta, \Xi$ denote arbitrary modifications in $L$ such that $\Xi \diamond \Theta$ exists. Then $\pi_{j}(\Xi \diamond \Theta)=(\Xi \diamond \Theta)_{j}(*)=\Xi_{j} \odot \Theta_{j}(*)=\Xi_{j}(*) \circ \Theta_{j}(*)=\pi_{j}(\Xi) \circ \pi_{j}(\Theta)$. Thus $\pi_{j}: L \rightarrow A_{j}$ is a functor.

Next I show that $\tau_{i, j}$ as defined above is a natural transformation for all $i, j \in$ $O b j \mathcal{J}$. By inspecting the definition diagram for $\tau_{i, j}$ above we see that for all $f \in \operatorname{Mor}_{\mathcal{J}}(i, j)$ we should have an element $\tau_{i, j}(f)$ of $\operatorname{MorMor}_{\mathcal{C}}\left(L, A_{j}\right)$. To this end, I claim that $\tau_{i, j}(f): F f \circ \pi_{i} \Rightarrow \pi_{j}$ is a natural transformation. To see this, let $\Theta: \eta \rightsquigarrow \eta^{\prime}$ be a modification, i.e. a morphism of the category $L$. Then by taking $\gamma=i_{f}$ in the definition of modification and evaluating the modification diagrams (11) and (2) at $* \in \operatorname{Obj} 1$ with $\alpha=\eta, \beta=\eta^{\prime}, A=i, B=j, \Xi=\Theta$ we obtain the commutivity of the diagram in the category $A_{j}$

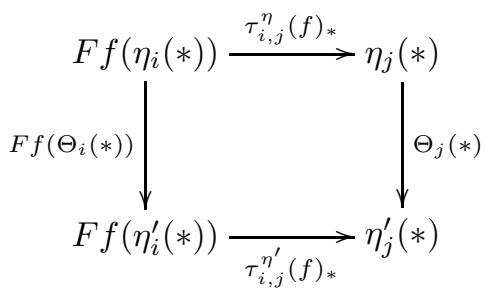

where $\tau^{\eta}$ and $\tau^{\eta^{\prime}}$ denote the coherence natural transformations belonging to the pseudo cones $\eta$ and $\eta^{\prime}$ respectively. Using the definitions $\tau_{i, j}(f)_{\eta}:=\tau_{i, j}^{\eta}(f)_{*}, \pi_{i}(\eta):=$ $\eta_{i}(*)$, and $\pi_{i}(\Theta):=\Theta_{i}(*)$ we see that this diagram is

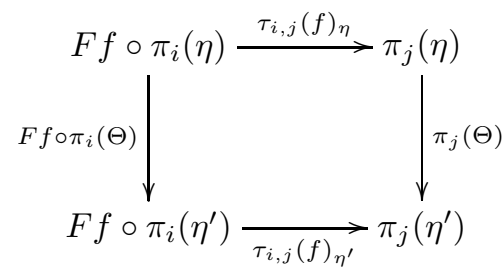

which says precisely that $\eta \mapsto \tau_{i, j}(f)_{\eta}$ is natural for fixed morphisms $f: i \rightarrow j$ of $\mathcal{J}$. Thus $\tau_{i, j}(f): F f \circ \pi_{i} \Rightarrow \pi_{j}$ is a natural transformation. On the other hand, the assignment $\operatorname{Mor}_{\mathcal{J}}(i, j) \ni f \mapsto \tau_{i, j}(f)$ is vacuously natural because the category $\operatorname{Mor}_{\mathcal{J}}(i, j)$ is discrete. Thus $\tau_{i, j}$ is a natural transformation for all $i, j \in O b j \mathcal{J}$.

The natural isomorphisms $\tau$ satisfy the unit axiom and composition axiom involving $\delta$ and $\gamma$ because the individual $\tau^{\eta}$ do.

Now I must show that the small category $L$ and the pseudo natural transformation $\pi: \Delta_{L} \Rightarrow F$ are universal in the sense that the functor $\phi: \operatorname{Mor}_{\mathcal{C}}(V, L) \rightarrow$ PseudoCone $(V, F)$ defined by $\phi b=\pi \circ b$ for objects $b$ is an isomorphism of categories for all objects $V$ of $\mathcal{C}$. More precisely, $\phi$ is defined for $b \in \operatorname{ObjMor}_{\mathcal{C}}(V, L)$ and $j \in O b j \mathcal{J}$ as $\phi(b)(j)=\pi_{j} \circ b$. The natural transformations for the pseudo cone $\phi b$ are $\tau_{i, j}(f) * i_{b}$ for all $f: i \rightarrow j$ in $\mathcal{J}$. For morphisms $\gamma: b \Rightarrow b^{\prime}$ in $\operatorname{MorMor}_{C}(V, L)$ I define $\phi(\gamma): \pi \circ b \rightsquigarrow \pi \circ b^{\prime}$ to be the modification which takes $j \in O b j \mathcal{J}$ to $\phi(\gamma)(j)=i_{\pi_{j}} * \gamma$. Here $i_{\pi_{j}}$ denotes as usual the identity 2-cell $\pi_{j} \Rightarrow \pi_{j}$ between these morphisms of $\mathcal{C}$. In the following, $V$ is a fixed object of the 2 -category $\mathcal{C}$ of small categories.

Lemma 4.3. The map $\phi: \operatorname{Mor}_{\mathcal{C}}(V, L) \rightarrow$ PseudoCone $(V, F)$ is a functor. 
Proof: The proof is analogous to the proof for the $\phi$ of the pseudo colimit.

Now I construct a functor $\psi: P \operatorname{seudoCone}(V, F) \rightarrow \operatorname{Mor}_{\mathcal{C}}(V, L)$ that is inverse to $\phi$. First I define $\psi$ for objects, then for morphism. Finally I verify that it is a functor and inverse to $\phi$. The key observation in the construction is that we can get a pseudo cone on a point by evaluating a pseudo cone on an object. This is the essence of the identification I make below.

Remark 4.4. Let $O b j P$ be the subset of the set $\left\{\left(a_{i}\right)_{i} \times\left(\varepsilon_{f}\right)_{f} \in \prod_{i \in O b j \mathcal{J}} \operatorname{Obj}_{i} \times \prod_{f \in M o r \mathcal{J}} M \operatorname{Mor} A_{T f} \mid \varepsilon_{f}: F f\left(a_{S f}\right) \rightarrow a_{T f}\right.$ is iso for all $f \in M$ or $\mathcal{J}\}$ consisting of all $\left(a_{i}\right)_{i} \times\left(\varepsilon_{f}\right)_{f}$ such that

- $\varepsilon_{1_{j}} \circ \delta_{j *}^{F}\left(a_{j}\right)=1_{a_{j}}$ for all $j \in O b j \mathcal{J}$

- $\varepsilon_{g} \circ\left(F g\left(\varepsilon_{f}\right)\right)=\varepsilon_{g \circ f} \circ \gamma_{f, g}^{F}\left(a_{S f}\right)$ for all $f, g \in M o r \mathcal{J}$ such that $g \circ f$ exists.

Then $\mathrm{Obj} L$ and $\mathrm{Obj} P$ are in bijective correspondence via the map $\mathrm{Obj} L \rightarrow O b j P, \eta \mapsto$ $\left(\eta_{i}(*)\right)_{i} \times\left(\tau_{S f, T f}^{\eta}(f)_{*}\right)_{f}$.

Proof: The two conditions express exactly the required coherences for a pseudo cone $\eta: \Delta_{1} \Rightarrow F$. Any pseudo cone $\eta: \Delta_{1} \Rightarrow F$ is completely determined by the data listed in the image sequence.

Remark 4.5. Let $\eta=\left(a_{i}\right)_{i} \times\left(\varepsilon_{f}\right)_{f}$ and $\eta^{\prime}=\left(a_{i}^{\prime}\right)_{i} \times\left(\varepsilon_{f}^{\prime}\right)_{f}$ be elements of ObjP. Let $\operatorname{Mor}_{P}\left(\eta, \eta^{\prime}\right)$ denote the set of $\left(\xi_{i}\right)_{i} \in \prod_{i \in O b j \mathcal{J}} \operatorname{Mor}_{A_{i}}\left(a_{i}, a_{i}^{\prime}\right)$ such that

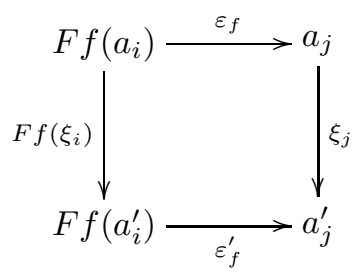

commutes for all $f: i \rightarrow j$ in $\mathcal{J}$. Then $\operatorname{Mor}_{L}\left(\eta, \eta^{\prime}\right)$ and $\operatorname{Mor}_{P}\left(\eta, \eta^{\prime}\right)$ are in bijective correspondence via the map $\operatorname{Mor}_{L}\left(\eta, \eta^{\prime}\right) \rightarrow \operatorname{Mor}_{P}\left(\eta, \eta^{\prime}\right), \Theta \mapsto\left(\Theta_{i}(*)\right)_{i}$. Moreover, the composition $\Theta \diamond \Xi$ in $\operatorname{Mor}_{L}\left(\eta, \eta^{\prime}\right)$ corresponds to the componentwise composition in $\operatorname{Mor}_{P}\left(\eta, \eta^{\prime}\right)$.

Proof: The diagram is the result of evaluating the coherence stated in diagrams (11) and (2) in the definition of modification at $*$. The claim about composition follows immediately from the definition of vertical composition $\diamond$ of modifications.

Remark 4.6. Under the identification above, $P$ is a category and $\pi_{j}$ is the projection onto the $j$-th coordinate.

Proof: $\quad$ This follows directly from the definition of $\pi$ and the identification.

I will use the identification without explanation. Now I define a functor $\psi\left(\pi^{\prime}\right)=$ $b: V \rightarrow L$ for any object $\pi^{\prime}$ of PseudoCone $(V, F)$. This will substantiate the comment that evaluating a pseudo cone on an object gives a pseudo cone on a point. 
Lemma 4.7. Let $\pi^{\prime}: \Delta_{V} \Rightarrow F$ be a pseudo natural transformation with coherence natural transformations $\tau^{\prime}$. For any fixed $x \in O b j V$ we have $\psi\left(\pi^{\prime}\right)(x):=b(x):=$ $\left(\pi_{i}^{\prime}(x)\right)_{i} \times\left(\tau_{S f, T f}^{\prime}(f)_{x}\right)_{f}$ is an element of $\operatorname{Obj} P=\operatorname{ObjL}$.

Proof: $\quad$ Evaluating the coherences for $\tau$ involving $\delta$ and $\gamma$ at the object $x$ gives the coherences in the definition of $P$. Thus $b(x) \in \operatorname{Obj} P$ and $b(x)$ is a pseudo cone $\Delta_{1} \Rightarrow F$, in other words $b(x)$ is a pseudo cone on a point.

Lemma 4.8. Let $\pi^{\prime}: \Delta_{V} \Rightarrow F$ be a pseudo natural transformation with coherence natural transformations $\tau^{\prime}$. Then for any fixed $h \in \operatorname{Mor}_{V}(x, y)$ we have a modification $\psi\left(\pi^{\prime}\right)(h):=b(h):=\left(\pi_{i}^{\prime}(h)\right)_{i}: b(x) \rightsquigarrow b(y)$. This notation means $b(h)_{i}(*):=\pi_{i}^{\prime}(h)$.

Proof: For notational convenience let $\eta:=b(x): \Delta_{\mathbf{1}} \Rightarrow F$ and $\eta^{\prime}:=b(y)$ : $\Delta_{1} \Rightarrow F$. Let $\Theta=b(h)$. Then $\tau_{i, j}^{\prime}(f)_{x}=\tau_{i, j}^{\eta}(f)_{*}$ and $\tau_{i, j}^{\prime}(f)_{y}=\tau_{i, j}^{\eta^{\prime}}(f)_{*}$ and $\Theta_{i}(*)=\pi_{i}^{\prime}(h)$ for all $f: i \rightarrow j$ in $\mathcal{J}$ by the identification. The naturality of $\tau_{i, j}^{\prime}(f)$ says $\tau_{i, j}^{\prime}(f)_{y} \circ F f\left(\pi_{i}^{\prime}(h)\right)=\pi_{j}^{\prime}(h) \circ \tau_{i, j}^{\prime}(f)_{x}$ for all $f: i \rightarrow j$ in $\mathcal{J}$. Rewriting this identity using $\eta, \eta^{\prime}$, and $\Theta$ gives $\tau_{i, j}^{\eta^{\prime}}(f)_{*} \circ F f\left(\Theta_{i}(*)\right)=\Theta_{j}(*) \circ \tau_{i, j}^{\eta}(f)_{*}$. This last identity says that the composition of natural transformations (2-cells)

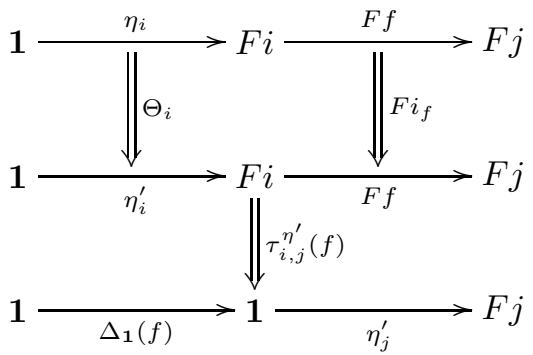

is the same as the composition

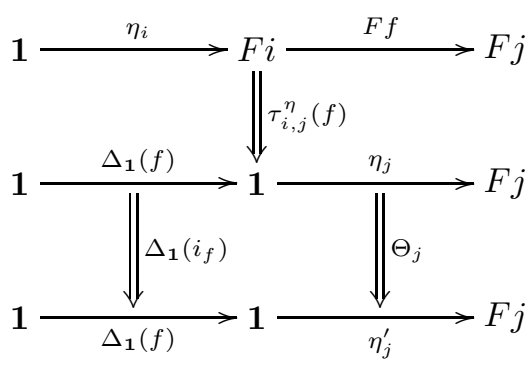

of natural transformations for all $f: i \rightarrow j$ in $\mathcal{J}$. The only 2-cells in the category $\mathcal{J}$ are of the form $i_{f}$. Therefore we have verified diagrams (11) and (2) for $\Theta$ to be a modification. Thus $\psi\left(\pi^{\prime}\right)(h)=b(h)=\Theta: \eta \rightsquigarrow \eta^{\prime}$ is a modification.

Lemma 4.9. For any pseudo natural transformation $\pi^{\prime}: \Delta_{V} \Rightarrow F$ the map $\psi\left(\pi^{\prime}\right)=$ $b: V \rightarrow L$ is a functor.

Proof: $\quad$ For each $x \in O b j V$ and all $j \in O b j \mathcal{J}$ we have $b\left(1_{x}\right)_{j}(*)=\pi_{j}^{\prime}\left(1_{x}\right)=1_{\pi_{j}^{\prime} x}$ since $\pi_{j}^{\prime}: V \rightarrow A_{j}$ is a functor. Hence $b\left(1_{x}\right)_{j}=i_{b(x)_{j}}$. Hence $b\left(1_{x}\right): b(x) \rightsquigarrow b(x)$ is the identity modification. If $h$ and $\ell$ are morphims in $V$ such that $\ell \circ h$ exists, then 
$b(\ell \circ h)_{j}(*)=\pi_{j}^{\prime}(\ell \circ h)=\pi_{j}^{\prime}(\ell) \circ \pi_{j}^{\prime}(h)=b(\ell)_{j}(*) \circ b(h)_{j}(*)=\left(b(\ell)_{j} \odot b(h)_{j}\right)(*)=$ $(b(\ell) \diamond b(h))_{j}(*)$. Hence $b(\ell \circ h)=b(\ell) \diamond b(h)$ and $b$ preserves compositions.

Lemma 4.10. Let $\Xi: \alpha \rightsquigarrow \beta$ be a morphism of the category PseudoCone $(V, F)$. Then $\psi(\Xi): \psi(\alpha) \Rightarrow \psi(\beta)$ defined by $V \ni x \mapsto\left(\Xi_{i}(x)\right)_{i} \in \operatorname{Mor}_{L}(\psi(\alpha) x, \psi(\beta) x)$ is a natural transformation. As in Remark 4.5 above, this definition means $\psi(\Xi)(x)_{i}(*):=$ $\Xi_{i}(x)$.

Proof: Since $\Xi: \alpha \rightsquigarrow \beta$ is a modification, for each object $i$ of $\mathcal{J}$ there is a 2-cell of $\mathcal{C}$ (a natural transformation) $\Xi_{i}: \alpha_{i} \Rightarrow \beta_{i}$ and these satisfy the coherence listed in the definition of modification. Evaluating this coherence in diagrams (1) and (2) at $x \in V$ we see that $\left(\Xi_{i}(x)\right)_{i}: \psi(\alpha) x \rightsquigarrow \psi(\beta) x$ is a modification. Hence $\left(\Xi_{i}(x)\right)_{i} \in \operatorname{Mor}_{L}(\psi(\alpha) x, \psi(\beta) x)$.

I claim that $\psi(\Xi)$ is natural, i.e. that the diagram

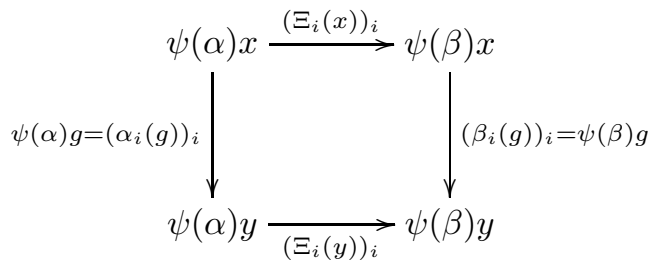

in $L$ commutes. We only need to verify that the diagram commutes componentwise, since the vertical composition of modifications corresponds to the componentwise composition of these sequences under the identification. But the diagram obviously commutes componentwise because $\Xi_{i}: \alpha_{i} \Rightarrow \beta_{i}$ is a natural transformation.

Theorem 4.11. The map $\psi:$ PseudoCone $(V, F) \rightarrow \operatorname{Mor}_{\mathcal{C}}(V, L)$ as defined in the previous lemmas is a functor.

Proof: $\quad$ Suppose $\Xi: \alpha \rightsquigarrow \alpha$ is the identity modification for a pseudo cone

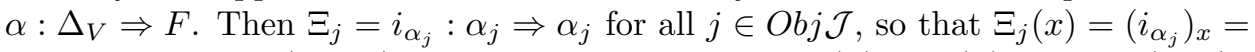
$1_{\alpha_{j}(x)}$. Then $x \mapsto\left(1_{\alpha_{j}(x)}\right)_{j}$ is the identity morphism $\psi(\alpha) \rightarrow \psi(\alpha)$ in $\operatorname{Mor}_{\mathcal{C}}(V, L)$.

If $\Xi, \Theta$ are modifications in PseudoCone $(V, F)$ such that $\Theta \diamond \Xi$ exists, then for all $x \in V$ we have $\psi(\Theta \diamond \Xi)(x)=\left((\Theta \diamond \Xi)_{i}(x)\right)_{i}$

$=\left(\left(\Theta_{i} \odot \Xi_{i}\right)(x)\right)_{i}$

$=\left(\Theta_{i}(x) \circ \Xi_{i}(x)\right)_{i}$

$=\left(\Theta_{i}(x)\right)_{i} \diamond\left(\Xi_{i}(x)\right)_{i}$

$=\psi(\Theta)(x) \diamond \psi(\Xi)(x)$

$=(\psi(\Theta) \odot \psi(\Xi))(x)$

Hence $\psi(\Theta \diamond \Xi)=\psi(\Theta) \odot \psi(\Xi)$ and $\psi$ preserves compositions.

Now that I have constructed the functor $\psi$, I prove that it is inverse to $\phi$.

Lemma 4.12. The functor $\psi$ is a left inverse for $\phi$, i.e. $\psi \circ \phi=1_{M o r_{\mathcal{C}}(V, L)}$.

Proof: First I verify the identity on objects. Let $b: V \rightarrow L$ be an object of $\operatorname{Mor}_{\mathcal{C}}(V, L)$. Recall that $\phi(b)$ is the pseudo natural transformation $\pi \circ b$ with the coherence natural transformations $\tau_{i, j}^{\prime}(f)=\tau_{i, j}(f) * i_{b}$ for all $f: i \rightarrow j$. For $x \in V$ 
we have $\psi \circ \phi(b)(x)=\psi(\pi \circ b)(x)$

$=\left(\pi_{i} \circ b(x)\right)_{i} \times\left(\tau_{S f, T f}^{\prime}(f)_{x}\right)_{f}$

$=\left(\pi_{i} \circ b(x)\right)_{i} \times\left(\left(\tau_{S f, T f}(f) * i_{b}\right)_{x}\right)_{f}$

$=\left(b(x)_{i}(*)\right)_{i} \times\left(\tau_{S f, T f}(f)_{b(x)}\right)_{f}$

$=\left(b(x)_{i}(*)\right)_{i} \times\left(\tau_{S f, T f}^{b(x)}(f)_{*}\right)_{f}$ by definition

$=b(x)$ by the identification.

For $g: x \rightarrow y$ in $V$ we have $\psi \circ \phi(b)(g)=\psi(\pi \circ b)(g)$

$=\left(\pi_{i} \circ b(g)\right)_{i}$

$=\left(b(g)_{i}(*)\right)_{i}$

$=b(g)$ by the identification.

Thus $\psi \circ \phi(b)$ and $b$ agree as functors.

Next I verify the identity on morphisms. Let $\gamma: b \Rightarrow b^{\prime}$ be a natural transformation. Then for $x \in V$ we have $\psi \circ \phi(\gamma)_{x}=\psi\left(i_{\pi} * \gamma\right)_{x}$

$=\left(\left(i_{\pi_{j}} * \gamma\right)_{x}\right)_{j}$

$=\left(\pi_{j}\left(\gamma_{x}\right)\right)_{j}$

$=\left(\gamma_{x j}(*)\right)_{j}$

$=\gamma_{x}$ by the identification.

Thus $\psi \circ \phi(\gamma)$ and $\gamma$ agree as natural transformations and $\psi \circ \phi=1_{M o r \mathcal{C}}(V, L)$.

Another way to see this is to notice that $\pi_{i}$ is the projection onto the $i$-th coordinate.

Lemma 4.13. The functor $\psi$ is a right inverse for $\phi$, i.e. $\phi \circ \psi=1_{P s e u d o C o n e}(V, F)$.

Proof: First I verify the identity on objects. Let $\pi^{\prime}: \Delta_{V} \Rightarrow F$ be a pseudo cone. For $j \in O b j \mathcal{J}$ and $x \in V$ we have $\left(\phi \circ \psi\left(\pi^{\prime}\right)\right)_{j}(x)=\left(\pi \circ \psi\left(\pi^{\prime}\right)\right)_{j}(x)$

$=\pi_{j} \circ \psi\left(\pi^{\prime}\right)(x)$

$=\pi_{j}\left(\left(\pi_{i}^{\prime}(x)\right)_{i} \times\left(\tau_{S f, T f}^{\prime}(f)_{x}\right)_{f}\right)$

$=\pi_{j}^{\prime}(x)$ because $\pi_{j}$ is basically projection onto the $j$-th coordinate under the identification.

Next I verify the identity on morphisms. Let $\Xi: \alpha \rightsquigarrow \beta$ be a modification in PseudoCone $(V, F)$. For $j \in O b j \mathcal{J}$ and $x \in V$ we have $(\phi \circ \psi(\Xi))_{j}(x)=\left(i_{\pi_{j}} * \psi(\Xi)\right)_{x}$ $=\pi_{j}\left(\psi(\Xi)_{x}\right)$

$=\pi_{j}\left(\left(\Xi_{i}(x)\right)_{i}\right)$

$=\Xi_{j}(x)$. Thus $\phi \circ \psi(\Xi)=\Xi$ and $\phi \circ \psi=1_{P s e u d o C o n e}(V, F)$.

Lemma 4.14. The small category $L$ with the pseudo cone $\pi: \Delta_{L} \Rightarrow F$ are a pseudo limit of the pseudo functor $F: \mathcal{J} \rightarrow \mathcal{C}$.

Proof: $\quad$ The functor $\phi: \operatorname{Mor}_{\mathcal{C}}(V, L) \rightarrow \operatorname{PseudoCone}(V, F)$ is an isomorphism of categories by the previous lemmas. Since $V$ was arbitrary we conclude that $L$ and $\pi$ are universal.

Thus every pseudo functor $F: \mathcal{J} \rightarrow \mathcal{C}$ from a small 1-category $\mathcal{J}$ to the 2category $\mathcal{C}$ of small categories has a pseudo limit. In other words, the 2-category $\mathcal{C}$ of small categories admits pseudo limits. This completes the proof of Theorem 4.1 .

Lemma 4.15. The 2-category $\mathcal{C}$ of small categories admits cotensor products. 
Proof: $\quad$ Let $J$ and $F$ be small categories. Then $\{J, F\}:=\mathcal{C}(J, F)$ is a cotensor product of $J$ and $F$ with unit $\pi: J \rightarrow \mathcal{C}(\mathcal{C}(J, F), F)$ defined by evaluation.

Theorem 4.16. The 2-category $\mathcal{C}$ of small categories admit all weighted pseudo limits.

Proof: This 2-category admits 2-products. It also admits cotensor products and pseudo equalizers by Lemma 4.15 and Theorem 4.1 Theorem 2.4 then implies that it admits all weighted pseudo limits.

Remark 4.17. The 2-category of small groupoids admits pseudo limits.

Proof: The proof is exactly the same as the proof for small categories, since $L=$ PseudoCone $(\mathbf{1}, F)$ is obviously a groupoid when the target of $F$ is the 2category of small groupoids.

Theorem 4.18. The 2-category of small categories and the 2-category of small groupoids admits bilimits.

Proof: They have pseudo limits, hence they also admit bilimits.

\section{Theories and Algebras}

The axioms for a group provide an example for the concept of a theory and an example of a group is an algebra over the theory of groups. In this section we describe what this means. Hu and Kriz point out in 24 that Lawvere's notion of a theory [34 is equivalent to another notion of theory. We prove this equivalence. It is well known that the category of algebras over a theory $T$ is equivalent to the category of algebras for some monad $C$. We present a version of this. Next we generalize the concept of theory in two ways. Firstly, we introduce theories on a set of objects. This allows one to describe algebraic structures on more than one set, such as modules or theories themselves. It also allows one to describe the free theory on a sequence of sets. Secondly, we introduce theories enriched in groupoids, which will be useful in the next section. Pseudo algebras over a theory $T$ are described in the next section as algebras over a theory $\mathcal{T}$ enriched in groupoids.

A theory can also be described as a finitary monad on Set as put forth in [7]. Theories on more than one object are called many-sorted in the monad description. Free finitary monads in the enriched and many-sorted contexts can be found in 30. and [33. See [48] for monads in a general 2-category.

Definition 5.1. A theory is a category $T$ with objects $0,1,2, \ldots$ such that $n$ is the product of 1 with itself $n$ times in the category $T$ and each $n$ is equipped with a limiting cone.

This definition means for each $n \in O b j T$ we have chosen morphisms $p r_{i}: n \rightarrow$ 1 for $i=1, \ldots, n$ with the universal property: for any object $m \in O b j T$ and morphisms $w_{i}: m \rightarrow 1$ for $i=1, \ldots, n$ there exists a unique morphism $\prod_{j=1}^{n} w_{j}: m \rightarrow n$ such that the diagram

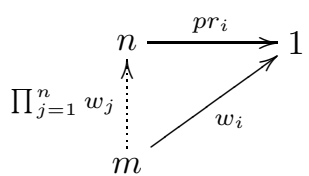


commutes for all $i=1, \ldots, n$. In particular 0 is the terminal object of the category $T$. Note that we do not require the projection $p r_{1}: 1 \rightarrow 1$ to be the identity, although it will automatically be an isomorphism. A useful notation is $T(n):=$ $\operatorname{Mor}_{T}(n, 1)$ for $n \in O b j T$. Elements of $T(n)$ are called words of arity $n$.

Another relevant morphism is the following. Let $\iota_{i}:\left\{1, \ldots, n_{i}\right\} \rightarrow\left\{1, \ldots, n_{1}+\right.$ $\left.n_{2}+\cdots+n_{k}\right\}$ be the injective map which takes the domain to the $i$-th block and suppose that $w_{i}: n_{i} \rightarrow 1$ for all $i=1, \ldots k$. Then there exists a unique map denoted $\left(w_{1}, \ldots, w_{k}\right)$ such that

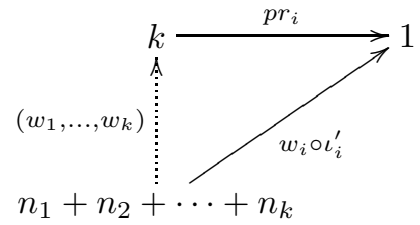

commutes for all $i=1, \ldots, k$ where $\iota^{\prime}: n_{1}+n_{2}+\cdots+n_{k} \rightarrow n_{i}$ is the unique morphism such that

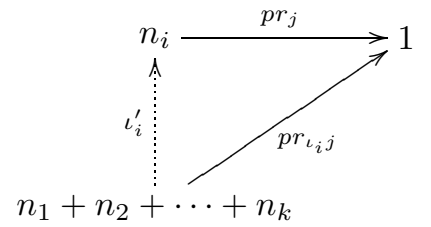

commutes. One should keep in mind that $n_{1}+n_{2}+\cdots+n_{k}$ is the product of $n_{1}, \ldots, n_{k}$. Note that $\left(w_{1}, \ldots, w_{k}\right)$ is not the same thing as the tuple $w_{1}, \ldots, w_{k}$. The arrow $\left(w_{1}, \ldots, w_{k}\right)$ is not the product of $w_{1}, \ldots, w_{k}$.

Lemma 5.1. Let $T$ be a theory. Then $\operatorname{Mor}_{T}(m, n)$ can be identified with the set product $\prod_{j=1}^{n} \operatorname{Mor}_{T}(m, 1)$ via the map which takes $w$ to the tuple with entries $p r_{1} \circ w, \ldots, p r_{n} \circ w$. We identify $w$ with that tuple. In particular a theory is determined up to isomorphism by the sets $T(0), T(1), T(2), \ldots$

Proof: This follows directly from the definition of product in a category.

Example 7. Let $X$ be a set. Then the endomorphism theory End( $X)$ has objects $0,1,2, \ldots$ and hom sets $\operatorname{Mor}_{\operatorname{End}(X)}(m, n)=\operatorname{Map}\left(X^{m}, X^{n}\right)$. Composition is the usual function composition. Here one readily sees that $\{*\}$ is the terminal object and that $\operatorname{End}(X)(0)=\operatorname{Mor}_{\operatorname{End}(X)}(0,1)$ can be identified with $X$.

Let $w \in \operatorname{End}(X)(k)$ and $w_{i} \in \operatorname{End}(X)\left(n_{i}\right)$ for $i=1, \ldots, k$. Then the composite function $\gamma\left(w, w_{1}, \ldots, w_{k}\right):=w \circ\left(w_{1}, \ldots, w_{k}\right)$ is an element of $\operatorname{End}(X)\left(n_{1}+\cdots+n_{k}\right)$. This composition is associative. Let $1:=1_{X} \in \operatorname{End}(X)(1)$. Then apparently $w \circ(1, \ldots, 1)=w$ and $1 \circ w=w$, i.e. the composition is also unital.

Let $\{1, \ldots, k\} \stackrel{f}{\longrightarrow}\{1, \ldots, \ell\} \stackrel{g}{\longrightarrow}\{1, \ldots, m\}$ be maps of sets. For $w \in \operatorname{End}(X)(k)$ we define a new word $w_{f} \in \operatorname{End}(X)(\ell)$ by $w_{f}\left(x_{1}, \ldots, x_{\ell}\right):=$ $w\left(x_{f 1}, \ldots, x_{f k}\right)$ called the substituted word. Thus we have maps

$\operatorname{End}(X)(k) \stackrel{\left(_{f}\right.}{\longrightarrow} \operatorname{End}(X)(\ell) \stackrel{()_{g}}{\longrightarrow} \operatorname{End}(X)(m)$. If $e: \emptyset \rightarrow\{1, \ldots, k\}$ is the empty function and $x \in X=\operatorname{End}(X)(0)$, then $x_{e}: X^{k} \rightarrow X$ is the constant function $\left(x_{1}, \ldots, x_{k}\right) \mapsto x$. There are no other functions $\emptyset \rightarrow\{1, \ldots, k\}$. One easily sees that $\left(w_{f}\right)_{g}=w_{g \circ f}$ and $w_{i d}=w$ for the identity map $i d:\{1, \ldots, k\} \rightarrow\{1, \ldots, k\}$, i.e. these substitution maps are functorial. 
These substitution maps relate to the composition in two ways. Let $f:\{1, \ldots, k\} \rightarrow$ $\{1, \ldots, \ell\}, w \in \operatorname{End}(X)(k)$, and $w_{i} \in \operatorname{End}(X)\left(n_{i}\right)$ for $i=1, \ldots, \ell$. Then $w_{f} \circ$ $\left(w_{1}, \ldots, w_{\ell}\right)=\left(w \circ\left(w_{f 1}, \ldots, w_{f k}\right)\right)_{\bar{f}}$ where $\bar{f}:\left\{1,2, \ldots, n_{f 1}+n_{f 2}+\cdots+n_{f k}\right\} \rightarrow$ $\left\{1,2, \ldots, n_{1}+n_{2}+\cdots+n_{\ell}\right\}$ is the function obtained by parsing the sequence $1,2, \ldots, n_{1}+n_{2}+\cdots+n_{\ell}$ into consecutive blocks $B_{1}, \ldots, B_{\ell}$ of lengths $n_{1}, \ldots, n_{\ell}$ respectively and then writing them in order $B_{f 1}, \ldots, B_{f k}$. For example, let $n_{1}=$ $1, n_{2}=2, n_{3}=3, n_{4}=1, w \in T(3)$, and $w_{i} \in T\left(n_{i}\right)$ for $i=1, \ldots, 4$ and let $f:\{1,2,3\} \rightarrow\{1,2,3,4\}$ be given by

$$
\left(\begin{array}{lll}
1 & 2 & 3 \\
3 & 2 & 4
\end{array}\right)
$$

Then $\bar{f}:\{1,2, \ldots, 6\} \rightarrow\{1,2, \ldots, 7\}$ is given by

$$
\left(\begin{array}{cccccc}
1 & 2 & 3 & 4 & 5 & 6 \\
B_{f 1} & & & B_{f 2} & & B_{f 3}
\end{array}\right)=\left(\begin{array}{cccccc}
1 & 2 & 3 & 4 & 5 & 6 \\
4 & 5 & 6 & 2 & 3 & 7
\end{array}\right) .
$$

We see that

$$
\begin{aligned}
w_{f} \circ\left(w_{1}, w_{2}, w_{3}, w_{4}\right)\left(x_{1}, \ldots, x_{7}\right) & =w_{f}\left(w_{1}\left(x_{1}\right), w_{2}\left(x_{2}, x_{3}\right), w_{3}\left(x_{4}, x_{5}, x_{6}\right), w_{4}\left(x_{7}\right)\right) \\
& =w\left(w_{3}\left(x_{4}, x_{5}, x_{6}\right), w_{2}\left(x_{2}, x_{3}\right), w_{4}\left(x_{7}\right)\right) \\
& =w \circ\left(w_{f 1}, w_{f 2}, w_{f 3}\right)\left(x_{4}, x_{5}, x_{6}, x_{2}, x_{3}, x_{7}\right) \\
& =w \circ\left(w_{f 1}, w_{f 2}, w_{f 3}\right)\left(x_{\bar{f} 1}, x_{\bar{f} 2}, \ldots, x_{\bar{f} 6}\right) .
\end{aligned}
$$

In other words we have $w_{f} \circ\left(w_{1}, w_{2}, w_{3}\right)=\left(w \circ\left(w_{f 1}, w_{f 2}, w_{f 3}\right)\right)_{\bar{f}}$. Note that $\bar{f}$ depends not only on $f$, but also on the arity of the words we are composing. The equality $w_{f} \circ\left(w_{1}, \ldots, w_{\ell}\right)=\left(w \circ\left(w_{f 1}, \ldots, w_{f k}\right)\right)_{\bar{f}}$ is the first relationship between composition and the substitution maps ()$_{f}$.

The second way the composition and the substitution maps relate occurs in the following situation. If $w \in \operatorname{End}(X)(k), w_{i} \in \operatorname{End}(X)\left(n_{i}\right)$, and $g_{i}:\left\{1, \ldots, n_{i}\right\} \rightarrow$ $\left\{1, \ldots, n_{i}^{\prime}\right\}$ are functions for $i=1, \ldots, k$, then $w \circ\left(\left(w_{1}\right)_{g_{1}}, \ldots,\left(w_{k}\right)_{g_{k}}\right)=(w \circ$ $\left.\left(w_{1}, \ldots, w_{k}\right)\right)_{g_{1}+\cdots+g_{k}}$ where $g_{1}+\cdots+g_{k}:\left\{1,2, \ldots, n_{1}+\cdots+n_{k}\right\} \rightarrow\left\{1,2, \ldots, n_{1}^{\prime}+\right.$ $\left.\cdots+n_{k}^{\prime}\right\}$ is the function obtained by placing $g_{1}, \ldots, g_{k}$ next to each other from left to right.

Example 8. Let $X$ be a category. Then the endomorphism theory $\operatorname{End}(X)$ has objects $0,1,2, \ldots$ and it has hom sets $\operatorname{Mor}_{\operatorname{End}(X)}(m, n)=$ Functors $\left(X^{m}, X^{n}\right)$. One can proceed as in the previous example and define substituted functors (substituted words). Note that $\operatorname{End}(X)$ can be made into a 2-category by taking the 2-cells to be natural transformations, although we leave out the 2-cells for now. In most applications we will only be concerned with the 1-category $\operatorname{End}(X)$.

Example 9. Let $X$ be an object of a category with finite products. Then we can get a theory $\operatorname{End}(X)$ by taking the hom sets to be $\operatorname{Mor}_{E n d(X)}(m, n)=\operatorname{Mor}\left(X^{m}, X^{n}\right)$.

We can abstract the essential properties of $\operatorname{End}(X)$ in the previous examples to get the following lemma for arbitrary theories.

Lemma 5.2. Let $T$ be a theory. Then for all $k, n_{1}, \ldots, n_{k} \in\{0,1, \ldots\}$ there is a map $\gamma: T(k) \times T\left(n_{1}\right) \times \cdots \times T\left(n_{k}\right) \rightarrow T\left(n_{1}+\cdots+n_{k}\right)$ called composition and for every function $f:\{1, \ldots, k\} \rightarrow\{1, \ldots, \ell\}$ there is a map $T(k) \stackrel{()_{f}}{\longrightarrow} T(\ell)$ called substitution. These maps have the following properties. 
(1) The $\gamma$ 's are associative, i.e.

$\gamma\left(w, \gamma\left(w^{1}, w_{1}^{1}, \ldots, w_{n_{1}}^{1}\right), \gamma\left(w^{2}, w_{1}^{2}, \ldots, w_{n_{2}}^{2}\right), \ldots, \gamma\left(w^{k}, w_{1}^{k}, \ldots, w_{n_{k}}^{k}\right)\right)$ is the same as

$\gamma\left(\gamma\left(w, w^{1}, \ldots, w^{k}\right), w_{1}^{1}, \ldots, w_{n_{1}}^{1}, w_{1}^{2}, \ldots, w_{n_{2}}^{2}, \ldots, w_{1}^{k}, \ldots, w_{n_{k}}^{k}\right)$.

(2) The $\gamma$ 's are unital, i.e. there exists an element $1 \in T(1)$ called the unit such that $\gamma(w, 1, \ldots, 1)=w=\gamma(1, w)$ for all $w \in T(k)$. Moreover, such an element is unique.

(3) The $\gamma$ 's are equivariant in the sense that $\gamma\left(w_{f}, w_{1}, \ldots, w_{\ell}\right)=\gamma\left(w, w_{f 1}, \ldots, w_{f k}\right)_{\bar{f}}$ for all $f:\{1, \ldots, k\} \rightarrow\{1, \ldots, \ell\}$ where $\bar{f}:\left\{1,2, \ldots, n_{f 1}+n_{f 2}+\cdots+n_{f k}\right\} \rightarrow\left\{1,2, \ldots, n_{1}+n_{2}+\cdots+n_{\ell}\right\}$ is the function that moves entire blocks according to $f$ as mentioned in the example above. Here $\bar{f}$ depends also on the particular $\gamma$.

(4) The $\gamma$ 's are equivariant in the sense that $\gamma\left(w,\left(w_{1}\right)_{g_{1}}, \ldots,\left(w_{k}\right)_{g_{k}}\right)=\gamma\left(w, w_{1}, \ldots, w_{k}\right)_{g_{1}+\cdots+g_{k}}$ for all functions $g_{i}$ : $\left\{1, \ldots, n_{i}\right\} \rightarrow\left\{1, \ldots, n_{i}^{\prime}\right\}$ where $g_{1}+\cdots+g_{k}:\left\{1,2, \ldots, n_{1}+\cdots+n_{k}\right\} \rightarrow$ $\left\{1,2, \ldots, n_{1}^{\prime}+\cdots+n_{k}^{\prime}\right\}$ is the function obtained by placing $g_{1}, \ldots, g_{k}$ next to each other from left to right.

(5) The substitution is functorial, i.e. for functions

$\{1, \ldots, k\} \stackrel{f}{\longrightarrow}\{1, \ldots, \ell\} \stackrel{g}{\longrightarrow}\{1, \ldots, m\}$ the composition

$T(k) \stackrel{()_{f}}{\longrightarrow} T(\ell) \stackrel{()_{g}}{\longrightarrow} T(m)$ is the same as

$T(k) \stackrel{()_{g \circ f}}{\longrightarrow} T(m)$ and for all $k$ the identity map $i d:\{1, \ldots, k\} \rightarrow\{1, \ldots, k\}$

induces the identity map $T(k) \stackrel{()_{i d}}{\longrightarrow} T(k)$.

Proof: First I define the substitution. Let $f:\{1, \ldots, k\} \rightarrow\{1, \ldots, \ell\}$ be a function. Then there exists a unique morphism $f^{\prime}$ such that the diagram

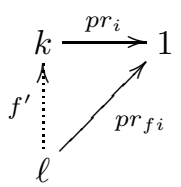

commutes for all $i=1, \ldots, k$. For $w \in T(k)$ define $w_{f}:=w \circ f^{\prime}$. Thus the map $T(k) \stackrel{()_{f}}{\longrightarrow} T(\ell)$ is defined by precomposition with $f^{\prime}$.

Next I define the composition $\gamma: T(k) \times T\left(n_{1}\right) \times \cdots \times T\left(n_{k}\right) \rightarrow T\left(n_{1}+n_{2}+\right.$ $\left.\cdots+n_{k}\right)$. Let $w \in T(k), w_{i} \in T\left(n_{i}\right)$ for $i=1, \ldots, k$. Define $\gamma\left(w, w_{1}, \ldots, w_{k}\right):=$ $w \circ\left(w_{1}, \ldots, w_{k}\right)$ where the composition $\circ$ is the composition of the category $T$ and $\left(w_{1}, \ldots, w_{k}\right)$ is the unique morphism such that

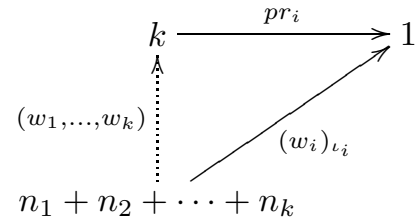

commutes as defined above.

(1) I claim that $\gamma$ is associative.

$$
\gamma\left(w, \gamma\left(w^{1}, w_{1}^{1}, \ldots, w_{n_{1}}^{1}\right), \gamma\left(w^{2}, w_{1}^{2}, \ldots, w_{n_{2}}^{2}\right), \ldots, \gamma\left(w^{k}, w_{1}^{k}, \ldots, w_{n_{k}}^{k}\right)\right)=
$$




$$
\begin{aligned}
& =w \circ\left(w^{1} \circ\left(w_{1}^{1}, \ldots, w_{n_{1}}^{1}\right), \ldots, w^{k} \circ\left(w_{1}^{k}, \ldots, w_{n_{k}}^{k}\right)\right) \\
& =w \circ\left(\left(w^{1}, \ldots, w^{k}\right) \circ\left(\left(w_{1}^{1}, \ldots, w_{n_{1}}^{1}\right), \ldots,\left(w_{1}^{k}, \ldots, w_{n_{k}}^{k}\right)\right)\right) \\
& =\left(w \circ\left(w^{1}, \ldots, w^{k}\right)\right) \circ\left(w_{1}^{1}, \ldots, w_{n_{1}}^{1}, \ldots, w_{1}^{k}, \ldots, w_{n_{k}}^{k}\right) \text { by associativity of } \\
& \text { composition in the category and by properties of products } \\
& =\gamma\left(\gamma\left(w, w^{1}, \ldots, w^{k}\right), w_{1}^{1}, \ldots, w_{n_{1}}^{1}, w_{1}^{2}, \ldots, w_{n_{2}}^{2}, \ldots, w_{1}^{k}, \ldots, w_{n_{k}}^{k}\right) .
\end{aligned}
$$

(2) I claim that $\gamma$ is unital. Let $1: 1 \rightarrow 1$ be the projection morphism of the object 1 in the category $T$, which is not necessarily the identity morphism of the object 1 . Then $(1, \ldots, 1): k \rightarrow k$ is the identity morphism of the object $k$ because $1_{\iota_{i}}=1 \circ\left(p r_{1}^{-1} \circ p r_{i}\right)=p r_{1} \circ\left(p r_{1}^{-1} \circ p r_{i}\right)=p r_{i}$ in the diagram

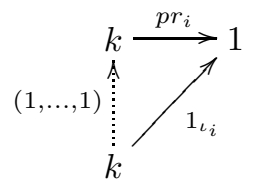

for all $i=1, \ldots, k$. Here $\iota_{i}:\{1\} \rightarrow\{1, \ldots, k\}$ is defined by $\iota_{i}(1)=i$. Thus $\gamma(w, 1, \ldots, 1)=w \circ(1, \ldots, 1)=w \circ 1_{k}=w$.

To show $\gamma(1, w)=w$ we consider the diagram

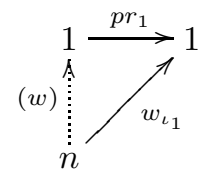

where $\iota_{1}:\{1, \ldots, n\} \rightarrow\{1, \ldots, n\}$ is the identity. Then $w_{\iota_{1}}=w$ and $(w)=p r_{1}^{-1} \circ w$. Thus $\gamma(1, w)=1 \circ(w)=p r_{1} \circ\left(p r_{1}^{-1} \circ w\right)=w$.

The uniqueness follows from $1=\gamma\left(1,1^{\prime}\right)=1^{\prime}$.

(3) Let $f:\{1, \ldots, k\} \rightarrow\{1, \ldots, \ell\}$ be a function and $w_{i} \in T(i)$ for $i=1, \ldots, \ell$. Using the definitions of $\bar{f}:\left\{1, \ldots, n_{f 1}+\cdots+n_{f k}\right\} \rightarrow\left\{1, \ldots, n_{1}+\cdots+n_{\ell}\right\}$ and $\iota_{i}$ from above we see that the following two diagrams
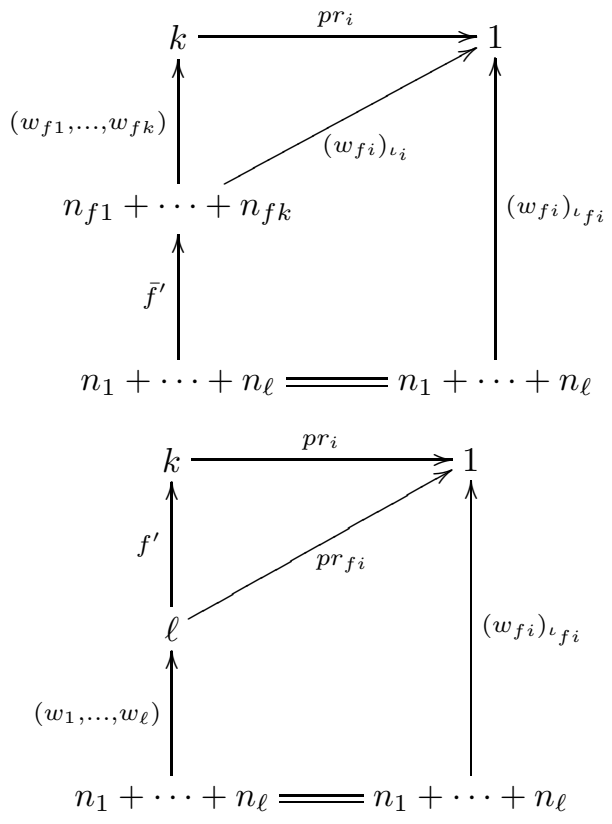
commute for all $i=1, \ldots, k$. Hence by the universal property of the product $k$ we have $f^{\prime} \circ\left(w_{1}, \ldots, w_{\ell}\right)=\left(w_{f 1}, \ldots, w_{f k}\right) \circ \bar{f}^{\prime}$. Using this we see that $\gamma\left(w_{f}, w_{1}, \ldots, w_{\ell}\right)=w \circ f^{\prime} \circ\left(w_{1}, \ldots, w_{\ell}\right)$ $=w \circ\left(w_{f 1}, \ldots, w_{f k}\right) \circ \bar{f}^{\prime}$ $=\gamma\left(w, w_{f 1}, \ldots, w_{f k}\right)_{\bar{f}}$.

(4) Let $g_{i}:\left\{1, \ldots, n_{i}\right\} \rightarrow\left\{1, \ldots, n_{i}^{\prime}\right\}$ be functions for $i=1, \ldots, k$. Then $\gamma\left(w,\left(w_{1}\right)_{g_{1}}, \ldots,\left(w_{k}\right)_{g_{k}}\right)=w \circ\left(w_{1} \circ g_{1}^{\prime}, \ldots, w_{k} \circ g_{k}^{\prime}\right)$ $=w \circ\left(w_{1}, \ldots, w_{k}\right) \circ\left(g_{1}^{\prime}, \ldots, g_{k}^{\prime}\right)$

$=w \circ\left(w_{1}, \ldots, w_{k}\right) \circ\left(g_{1}+\cdots+g_{k}\right)^{\prime}$

$=\gamma\left(w, w_{1}, \ldots, w_{k}\right)_{g_{1}+\cdots+g_{k}}$.

(5) Let $\{1, \ldots, k\} \stackrel{f}{\longrightarrow}\{1, \ldots, \ell\} \stackrel{g}{\longrightarrow}\{1, \ldots, m\}$ be functions. Then $f^{\prime}$ and $g^{\prime}$ make the two small subdiagrams in

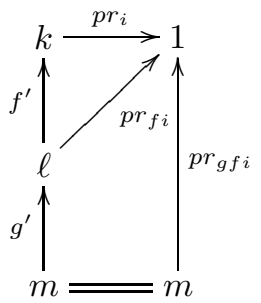

commute for all $i=1, \ldots, k$. Thus the outer diagram commutes and $g \circ$ $f)^{\prime}=f^{\prime} \circ g^{\prime}$ by the universal property of the product. We conclude $\left(w_{f}\right)_{g}=$ $w \circ f^{\prime} \circ g^{\prime}=w \circ(g \circ f)^{\prime}=w_{g \circ f}$. The identity $1_{k}: k \rightarrow k$ makes

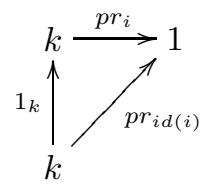

commute for all $i=1, \ldots, k$ where $i d:\{1, \ldots, k\} \rightarrow\{1, \ldots, k\}$ is the identity function. Hence $(i d)^{\prime}=1_{k}$ and $w_{i d}=w \circ(i d)^{\prime}=w \circ 1_{k}=w$ for all $w \in T(k)$.

We have verified all of the axioms.

There is another description of a theory which can be formulated by using the category $\Gamma$.

Definition 5.2. Let $\Gamma$ be the category with objects $\emptyset=0,1,2, \ldots$ where $k=$ $\{1, \ldots, k\}$. The morphisms $k \rightarrow \ell$ are just maps of sets. In particular 0 is the initial object since the only map $\emptyset \rightarrow k$ is the empty function. There are no maps $k \rightarrow \emptyset$ for $k \geq 1$. The object 1 is the terminal object. Let $+: \Gamma \times \Gamma \rightarrow \Gamma$ denote the usual functor obtained by adding the sets and placing maps side by side.

Remark 5.1. Let $T$ be a theory. Then by the previous lemma $T$ defines a functor from $\Gamma$ to Sets by $k \mapsto T(k)$ and $f \mapsto()_{f}$. Morover, this functor comes with maps $\gamma: T(k) \times T\left(n_{1}\right) \times \cdots \times T\left(n_{k}\right) \rightarrow T\left(n_{1}+\cdots+n_{k}\right)$ which satisfy 1 . through 5 . The compositions $\gamma$, unit 1 , and substitution are sometimes called the operations of theories. The relations in 1 . through 5 . are sometimes called the relations of theories. 
Lemma 5.3. Let $T$ be a functor from $\Gamma$ to Sets equipped with maps $\gamma: T(k) \times$ $T\left(n_{1}\right) \times \cdots \times T\left(n_{k}\right) \rightarrow T\left(n_{1}+\cdots+n_{k}\right)$ and an element $1 \in T(1)$ which satisfy (1) through (5) where $T(f)=:()_{f}$ for functions $f: k \rightarrow \ell$. Then $T$ determines a theory with $\operatorname{Mor}(n, 1)=T(n)$ for all $n \geq 0$.

Proof: Define the underlying category of the theory to formally have objects $0,1,2 \ldots$ and morphisms $\operatorname{Mor}(m, n):=\prod_{i=1}^{n} \operatorname{Mor}(m, 1)$. In particular Mor $(m, 0)$ only has one element. We denote a tuple of words $w_{1}, \ldots, w_{n} \in \operatorname{Mor}(m, 1)$ by $\prod_{i=1}^{n} w_{i}$. For $k, \ell \geq 0$ let $\iota_{\ell, k}:\{1, \ldots, \ell k\} \rightarrow\{1, \ldots, k\}$ be the function such that $\iota_{\ell, k}(i+j k)=i$ for $i=1, \ldots, k$, in other words $\iota_{\ell, k}$ wraps the domain around the codomain $\ell$ times. Now define the composition of $\prod_{i=1}^{\ell} w_{i} \in \operatorname{Mor}(k, \ell)$ with $\prod_{i=1}^{m} v_{i} \in \operatorname{Mor}(\ell, m)$ to be

$\prod_{i=1}^{m} v_{i} \circ \prod_{i=1}^{\ell} w_{i}:=\prod_{i=1}^{m} \gamma\left(v_{i}, w_{1}, \ldots, w_{\ell}\right)_{\iota_{\ell, k}}$. This composition is associative because $\gamma$ is associative and equivariant.

Let $f_{i}:\{1\} \rightarrow\{1, \ldots, n\}$ be the map $f_{i}(1)=i$. Define $p r_{i}:=1_{f_{i}} \in T(n)$ where $1 \in T(1)$ is the distinguished element whose existence is guaranteed by the axioms. This notation is slightly imprecise because I have different sequences $p r_{1}, \ldots, p r_{n}$ for different $n \geq 0$. From the context it will always be clear which sequence of morphisms is meant. I claim that $\prod_{i=1}^{n} p r_{i} \in \operatorname{Mor}(n, n)$ is the identity on the object $n$. Let $\prod_{i=1}^{m} w_{i} \in \operatorname{Mor}(n, m)$. Then

$\prod_{i=1}^{m} w_{i} \circ \prod_{i=1}^{n} p r_{i}=\prod_{i=1}^{m} \gamma\left(w_{i}, p r_{1}, \ldots, p r_{n}\right)_{\iota_{n, n}}$

$=\prod_{i=1}^{m} \gamma\left(w_{i}, 1_{f_{1}}, \ldots, 1_{f_{n}}\right)_{\iota_{n, n}}$

$=\prod_{i=1}^{m}\left(\gamma\left(w_{i}, 1, \ldots, 1\right)_{f_{1}+\cdots+f_{n}}\right)_{\iota_{n, n}}$ by equivariance

$=\prod_{i=1}^{m} \gamma\left(w_{i}, 1, \ldots, 1\right)_{\iota_{n, n} \circ\left(f_{1}+\cdots+f_{n}\right)}$ by functoriality of $T$

$=\prod_{i=1}^{m} w_{i}$ by unitality of $\gamma$ and $\iota_{n, n} \circ\left(f_{1}+\cdots+f_{n}\right)=1_{\{1, \ldots, n\}}$ and also functoriality of $T$. Now for the other side let $\prod_{i=1}^{n} w_{i} \in \operatorname{Mor}(m, n)$. Then

$\prod_{i=1}^{n} p r_{i} \circ \prod_{i=1}^{n} w_{i}=\prod_{i=1}^{n} \gamma\left(p r_{i}, w_{1}, \ldots, w_{n}\right)_{\iota_{n, m}}$ by definition

$=\prod_{i=1}^{n} \gamma\left(1_{f_{i}}, w_{1}, \ldots, w_{n}\right)_{\iota_{n, m}}$ by definition

$=\prod_{i=1}^{n}\left(\gamma\left(1, w_{i}\right)_{\bar{f}_{i}}\right)_{\iota_{n, m}}$ by equivariance

$=\prod_{i=1}^{n}\left(w_{i}\right)_{\iota_{n, m} \circ \bar{f}_{i}}$ by unitality of $\gamma$ and functoriality of $T$

$=\prod_{i=1}^{n} w_{i}$ since $\iota_{n, m} \circ \bar{f}_{i}=1_{\{1, \ldots, m\}}$. This can be seen by observing that $\bar{f}_{i}$ : $\{1, \ldots, m\} \rightarrow\{1, \ldots, n m\}$ has the form

$$
\left(\begin{array}{cccc}
1 & 2 & \cdots & m \\
(i-1) m+1 & (i-1) m+2 & \cdots & (i-1) m+m
\end{array}\right)
$$

and by using the definition of $\iota_{n, m}$. Thus $\prod_{i=1}^{n} p r_{i} \in \operatorname{Mor}(n, n)$ is the identity on the object $n$.

Thus far we have shown that we have a category with objects $0,1,2, \ldots$ and morphisms $\operatorname{Mor}(m, n)$. I claim that $n$ is the product of $n$ copies of 1 in this category with projections $p r_{1}, \ldots, p r_{n}: n \rightarrow 1$ introduced above. First note for $\prod_{i=1}^{n} w_{i} \in \operatorname{Mor}(m, n)$ we have

$\operatorname{pr}_{i} \circ \prod_{i=1}^{n} w_{i}=\gamma\left(p r_{i}, w_{1}, \ldots, w_{n}\right)_{\iota_{n, m}}$ $=\gamma\left(1_{f_{i}}, w_{1}, \ldots, w_{n}\right)_{\iota_{n, m}}$ by definition

$=\gamma\left(1, w_{i}\right)_{\iota_{n, m} \circ \bar{f}_{i}}$ by equivariance and functoriality

$=w_{i}$ by $\iota_{n, m} \circ \bar{f}_{i}=1_{\{1, \ldots, m\}}$ and functoriality. 
Now suppose we are given morphisms $w_{1}, \ldots, w_{n} \in \operatorname{Mor}(m, 1)$. Then

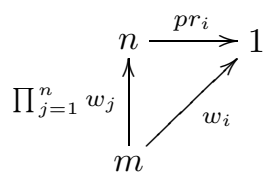

commutes for all $i=1, \ldots, n$ by the remark just made. If $\prod_{i=1}^{n} v_{i} \in \operatorname{Mor}(m, n)$ is another morphism such that

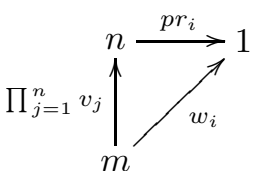

commutes for all $i=1, \ldots, n$, then by the remark $v_{i}=p r_{i} \circ \prod_{j=1}^{n} v_{j}=w_{i}$ and hence $\prod_{j=1}^{n} v_{j}=\prod_{j=1}^{n} w_{j}$ and the factorizing map is unique. Hence $n$ is the product of $n$ copies of 1 .

We conclude that the functor $T$ with the maps $\gamma$ satisfying the axioms (1) through (5) determines a theory with the indicated hom sets.

Theorem 5.4. A theory $T$ is determined by either of the following equivalent collections of data:

(1) A category $T$ with objects $0,1,2, \ldots$ such that $n$ is the categorical product of 1 with itself $n$ times and each $n$ is equipped with a choice of projections

(2) A functor $T: \Gamma \rightarrow$ Sets equipped with maps $\gamma: T(k) \times T\left(n_{1}\right) \times \cdots \times T\left(n_{k}\right) \rightarrow$ $T\left(n_{1}+\cdots+n_{k}\right)$ and a unit $1 \in T(1)$ which satisfy (1) through (5) of Lemma 5.2.

Proof: In each description $\operatorname{Mor}_{T}(n, 1)$ is the same. By the universality of products this determines the rest of the theory. The two processes of Lemmas 5.2 and 5.3 are inverse to one another by further inspection, provided one identifies $\operatorname{Mor}_{T}(m, n)$ with $\prod_{i=1}^{n} T(m)$.

Definition 5.3. Let $S$ and $T$ be theories. In the categorical description of $S$ and $T$ a morphism of theories $\Phi: S \rightarrow T$ is a functor from the category $S$ to the category $T$ such that $\Phi\left(n_{S}\right)=n_{T}$ and $\Phi\left(p r_{i}\right)=p r_{i}$ for all projections.

One easily sees that the theories form a category and we have a suitable forgetful functor.

Theorem 5.5. The forgetful functor from the category of theories to $\prod_{n \geq 0}$ Sets given by $T \mapsto(T(0), T(1), \ldots)$ has a left adjoint called the free theory functor.

Proof: Later we will construct the free theory on a sequence of sets $(T(0), T(1), \ldots)$.

To make later proofs easier, we need the following lemma.

Lemma 5.6. Let $\Phi: S \rightarrow T$ be a morphism of theories. 
(1) Let $f:\{1, \ldots, k\} \rightarrow\{1, \ldots, \ell\}$ be a function. As usual, $f^{\prime}: \ell \rightarrow k$ denotes the unique morphism in any theory such that

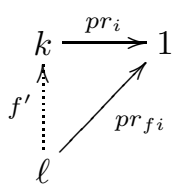

commutes. Then $\Phi\left(f^{\prime}\right)=f^{\prime}$.

(2) Let $f:\{1, \ldots, k\} \rightarrow\{1, \ldots, \ell\}$ be a function and $w \in \operatorname{Mor}_{S}(k, 1)$. Then $\Phi\left(w_{f}\right)=\Phi(w)_{f}$.

(3) Let $w_{1}, \ldots, w_{n} \in \operatorname{Mor}_{S}(m, 1)$. Then $\Phi\left(\prod_{j=1}^{n} w_{j}\right)=\prod_{j=1}^{n} \Phi\left(w_{j}\right)$.

(4) Let $w_{i} \in \operatorname{Mor}_{S}\left(n_{i}, 1\right)$ for $i=1, \ldots, k$. Then $\Phi\left(w_{1}, \ldots, w_{k}\right)=\left(\Phi\left(w_{1}\right), \ldots, \Phi\left(w_{k}\right)\right)$.

Proof:

(1) The diagram

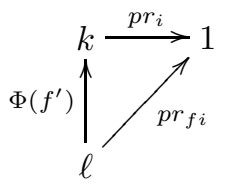

commutes for all $i=1, \ldots, k$ by the properties of $\Phi$. Then $\Phi\left(f^{\prime}\right)=f^{\prime}$ by the universal property of the product.

(2) This follows from (1) and the definition $w_{f}=w \circ f^{\prime}$.

(3) The properties of $\Phi$ imply that the diagram

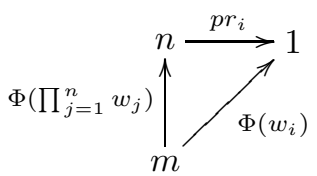

commutes for all $i=1, \ldots, n$. Then $\Phi\left(\prod_{j=1}^{n} w_{j}\right)=\prod_{j=1}^{n} \Phi\left(w_{j}\right)$ by the universal property of the product.

(4) By (2) we have $\Phi\left(\left(w_{i}\right)_{\iota_{i}}\right)=\Phi\left(w_{i}\right)_{\iota_{i}}$. Hence, the properties of $\Phi$ imply that the diagram

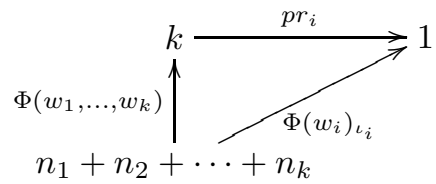

commutes for all $i=1, \ldots, k$. Then $\Phi\left(w_{1}, \ldots, w_{k}\right)=\left(\Phi\left(w_{1}\right), \ldots, \Phi\left(w_{k}\right)\right)$ by the universal property of the product.

Just as a theory has a categorical description and a functorial description, a morphism of theories also has a second description. We work towards the second description in the following two lemmas.

Lemma 5.7. Let $\Phi: S \rightarrow T$ be a morphism of theories, i.e. a functor such that $\Phi\left(n_{S}\right)=n_{T}$ and $\Phi\left(p r_{i}\right)=p r_{i}$ for all projections. Then these data determine a natural transformation $S \Rightarrow T$ also denoted by $\Phi$ such that 


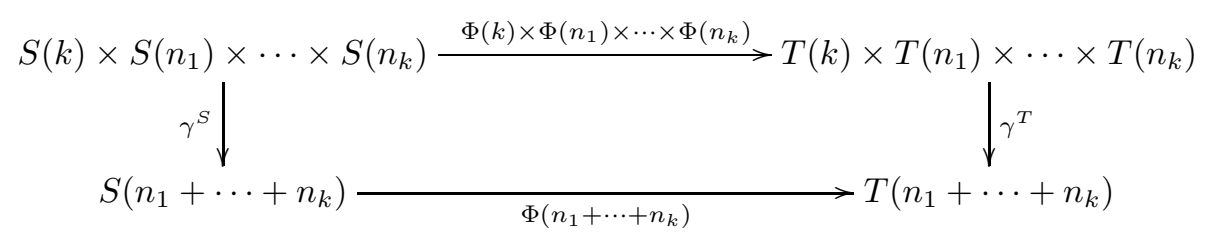

commutes and $\Phi(1)\left(1_{S}\right)=1_{T}$, where $S, T: \Gamma \Rightarrow$ Sets are the functors in the functorial description of the theories $S$ and $T$.

Proof: $\quad$ Let $\Phi(m): \operatorname{Mor}_{S}(m, 1) \rightarrow \operatorname{Mor}_{T}(m, 1)$ denote the map obtained from the functor $\Phi$, i.e. $\Phi(m)(w):=\Phi(w)$ for $w \in S(m)$. Then for $f: m \rightarrow n$ in $\Gamma$ and $w \in S(m)$, we have $\Phi\left(w_{f}\right)=\Phi(w)_{f}$ by the previous lemma. Hence

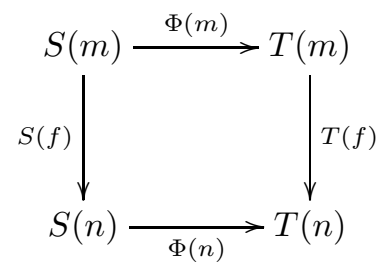

commutes and $m \mapsto \Phi(m)$ is natural.

Let $w \in S(k), w_{i}$ for $i=1, \ldots, k$. Then

$$
\begin{aligned}
\Phi\left(n_{1}+\cdots+n_{k}\right)\left(\gamma^{S}\left(w, w_{1}, \ldots, w_{k}\right)\right) & =\Phi\left(w \circ\left(w_{1}, \ldots, w_{k}\right)\right) \\
& =\Phi(w) \circ\left(\Phi\left(w_{1}\right), \ldots, \Phi\left(w_{k}\right)\right) \\
& =\gamma^{T}\left(\Phi(w), \Phi\left(w_{1}\right), \ldots, \Phi\left(w_{k}\right)\right) \\
& =\gamma^{T}\left(\Phi(k)(w), \Phi\left(n_{1}\right)\left(w_{1}\right), \ldots, \Phi\left(n_{k}\right)\left(w_{k}\right)\right) .
\end{aligned}
$$

Hence the natural transformation $m \mapsto \Phi(m)$ preserves the $\gamma$ 's.

Let $1_{S} \in S(1)$ and $1_{T} \in T(1)$ be the units in the respective theories. Then $\Phi(1)\left(1_{S}\right)=1_{T}$ because the functor $\Phi$ preserves identities of the categories.

Thus $\Phi: S \Rightarrow T$ is a natural transformation which preserves the compositions and the units.

Lemma 5.8. Let $S, T: \Gamma \rightarrow$ Sets be theories. Let $\Phi: S \Rightarrow T$ be a natural transformation preserving the $\gamma$ 's and their units as in the proof of the previous lemma. Then these data determine a functor $S \rightarrow T$ also denoted $\Phi$, where $S$ and $T$ are the categories in the categorical description of the theories $S, T: \Gamma \rightarrow$ Sets. Moreover, the functor $\Phi: S \rightarrow T$ satisfies $\Phi\left(n_{S}\right)=n_{T}$ and $\Phi\left(p r_{i}\right)=p r_{i}$ for all projections.

Proof: $\quad$ Define $\Phi\left(n_{S}\right)=n_{T}$ for all $n_{S} \in O b j S$ and $\Phi\left(\prod_{j=1}^{\ell} w_{j}\right):=\prod_{j=1}^{\ell} \Phi(k)\left(w_{j}\right)$ for all $\prod_{j=1}^{\ell} w_{j} \in \operatorname{Mor}_{S}(k, \ell)$. Then for $\prod_{i=1}^{m} v_{i} \in \operatorname{Mor}_{S}(\ell, m)$ we have 


$$
\begin{aligned}
\Phi\left(\prod_{i=1}^{m} v_{i} \circ \prod_{j=1}^{\ell} w_{j}\right) & =\Phi\left(\prod_{i=1}^{m} \gamma\left(v_{i}, w_{1}, \ldots, w_{\ell}\right)_{\iota_{\ell, k}}\right) \text { from Lemma } 5.3 \\
& =\prod_{i=1}^{m} \gamma\left(\Phi(\ell)\left(v_{i}\right), \Phi(k)\left(w_{1}\right), \ldots, \Phi(k)\left(w_{\ell}\right)\right)_{\iota_{\ell, k}} \\
& =\prod_{i=1}^{m} \Phi(\ell)\left(v_{j}\right) \circ \prod_{j=1}^{\ell} \Phi(k)\left(w_{j}\right) \\
& =\Phi\left(\prod_{i=1}^{m} v_{i}\right) \circ \Phi\left(\prod_{j=1}^{\ell} w_{j}\right) .
\end{aligned}
$$

Hence $\Phi$ preserves compositions.

I claim that $\Phi$ preserves projections. Let $f_{i}:\{1\} \rightarrow\{1, \ldots, n\}$ be the map $f_{i}(1)=i$. Then $\left(1_{S}\right)_{f_{i}}=p r_{i}$ and

$$
\begin{aligned}
\Phi\left(p r_{i}\right) & =\Phi(n)\left(\left(1_{S}\right)_{f_{1}}\right) \\
& =\Phi(n)\left(1_{S}\right)_{f_{i}} \text { by naturality } \\
& =\left(1_{T}\right)_{f_{i}} \text { since } \Phi \text { preserves the unit } \\
& =p r_{i} .
\end{aligned}
$$

Hence $\Phi$ preserves projections.

I claim that $\Phi$ preserves identities. Recall that $\prod_{j=1}^{n} p r_{j}: n \rightarrow n$ is the identity on the object $n$ of the category $S$. Then

$$
\begin{aligned}
\Phi\left(\prod_{j=1}^{n} p r_{j}\right) & =\prod_{j=1}^{n} \Phi\left(p r_{j}\right) \text { by definition } \\
& =\prod_{j=1}^{n} p r_{j} \text { because } \Phi \text { preserves projections. }
\end{aligned}
$$

Thus $\Phi$ preserves identites and is a functor $S \rightarrow T$.

Combining these two lemmas gives us the two descriptions of a morphism of theories in the following theorem.

Theorem 5.9. Let $S$ and $T$ be theories. Then a morphism $S \rightarrow T$ of theories is given by either of the following equivalent collections of data:

(1) A functor $\Phi: S \rightarrow T$ such that $\Phi\left(n_{S}\right)=n_{T}$ for all $n_{S} \in$ ObjS and $\Phi\left(p r_{i}\right)=p r_{i}$ for all projections

(2) A natural transformation $\Phi: S \Rightarrow T$ of the functors $S, T: \Gamma \rightarrow$ Sets which preserves the $\gamma$ 's and the units.

Proof: The processes of the previous two lemmas are "inverse" to each other by inspection.

Theorem 5.10. The category of theories with objects and morphisms as in (1) of Theorems 5.4 and 5.9 is equivalent to the category with objects and morphisms as in (2) of Theorems 5.4 and 5.9 . 
The concept of an algebra is closely related to the concept of theories. Roughly speaking, an algebra over a theory is a category together with a rule that assigns an $n$-ary operation on $X$ to every word of the theory of arity $n$ in such way that compositions, substitutions, and identity 1 are preserved.

Definition 5.4. Let $X$ be a category and $T$ a theory. Then $X$ is a $T$-algebra if it is equipped with a morphism of theories $T \rightarrow \operatorname{End}(X)$, where $\operatorname{End}(X)$ is the theory in Example 8. We also say $X$ is an algebra over the theory $T$.

Notice that if $X$ is a set viewed as a discrete category, this is the usual definition of an algebra over a theory. For example, a group is an algebra over the theory of groups as follows.

Example 10. Let $T$ be the theory of groups, i.e. there are morphisms $e \in T(0), \nu \in$ $T(1)$, and $\mu \in T(2)$ which satisfy the usual group axioms. The theory $T$ is the largest theory containing such $e, \nu, \mu$. A set $X$ is a group if there is a morphism of theories $T \rightarrow \operatorname{End}(X)$. This means we have realizations of $e, \nu$, and $\mu$ on $X$.

Definition 5.5. Let $X$ and $Y$ be $T$-algebras. Then a functor $H: X \rightarrow Y$ is a morphism of $T$-algebras if

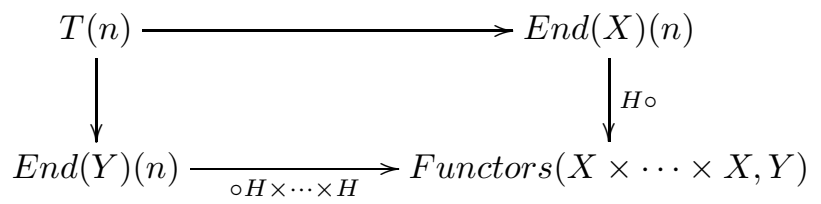

commutes for all $n$.

Example 11. Let $T$ be the theory of groups and let $X$ and $Y$ be groups. Then a set map $H: X \rightarrow Y$ is a morphism of $T$-algebras if and only if it is a group homomorphism.

Let $T$ be any theory. It is well known that $T$-algebras are algebras for a monad $C$, which depends on $T$. See for example [39] or 43]. I now present a version of this in preparation for the 2-monad whose strict algebras are pseudo $T$-algebras. Let $\mathrm{Cat}_{0}$ denote the 1-category of small categories. Let $T$ be a theory. Define a functor $C: \mathrm{Cat}_{0} \rightarrow \mathrm{Cat}_{0}$ as follows. For a small category $X$, set

$$
\operatorname{Obj} C X:=\frac{\left(\bigcup_{n \geq 0}\left(T(n) \times O b j X^{n}\right)\right)}{\Gamma}
$$

where we mod out by the smallest equivalence relation satisfying $\left(w_{f}, x_{1}, \ldots, x_{n}\right) \sim$ $\left(w, x_{f 1}, \ldots, x_{f m}\right)$ for all $m \in \mathbb{N}_{0}, w \in T(m)$, and maps $f: m=\{1, \ldots, m\} \rightarrow$ $\{1, \ldots, n\}=n$. To define the morphisms of $C X$ we note that $\bigcup_{n \geq 0}\left(T(n) \times X^{n}\right)$ is a category if we interpret $T(n)$ as a discrete category for each $n$. Consider the directed graph with objects $\operatorname{Obj} C X$ and arrows from $[a]$ to $[b]$ given by the union

$$
\bigcup \operatorname{Mor}_{\cup_{n \geq 0}\left(T(n) \times X^{n}\right)}\left(a^{\prime}, b^{\prime}\right)
$$

over all $a^{\prime} \sim a$ and $b^{\prime} \sim b$. Next take the free category on this directed graph and mod out by the relations of $\bigcup_{n \geq 0}\left(T(n) \times X^{n}\right)$ and the relations

$$
\left(i_{w_{f}}, g_{1}, \ldots, g_{n}\right)=\left(i_{w}, g_{f 1}, \ldots, g_{f m}\right) \text {. }
$$

This quotient category is $C X$. One defines $C$ on functors $X \rightarrow Y$ analogously. Then $C: \mathrm{Cat}_{0} \rightarrow \mathrm{Cat}_{0}$ is a functor because each step in the construction is functorial. 
Next define a natural transformation $\eta: 1_{C a t_{0}} \Rightarrow C$ by $\eta_{X}(x):=[1, x]$ for $x \in \operatorname{Obj} X$ and $\eta_{X}(g):=\left[i_{1}, g\right]$ for a morphism $g$ in $X$. Also define a natural transformation $\mu: C^{2} \Rightarrow C$ by

$$
\begin{gathered}
\mu_{X}\left(\left[w,\left[v^{1}, x_{1}^{1}, \ldots, x_{j_{1}}^{1}\right],\left[v^{2}, x_{1}^{2}, \ldots, x_{j_{2}}^{2}\right], \ldots,\left[v^{k}, x_{1}^{k}, \ldots, x_{j_{k}}^{k}\right]\right]\right):= \\
{\left[\gamma\left(w, v^{1}, v^{2}, \ldots, v^{k}\right), x_{1}^{1}, \ldots, x_{j_{k}}^{k}\right]}
\end{gathered}
$$

for $w \in T(k), v^{i} \in T\left(j_{i}\right)$, and $\left(x_{1}^{i}, \ldots, x_{j_{i}}^{i}\right) \in X^{j_{i}}$ for $i=1, \ldots, k$. On morphisms we define it to be

$$
\begin{gathered}
\mu_{X}\left(\left[i_{w},\left[i_{v^{1}}, g_{1}^{1}, \ldots, g_{j_{1}}^{1}\right],\left[i_{v^{2}}, g_{1}^{2}, \ldots, g_{j_{2}}^{2}\right], \ldots,\left[i_{v^{k}}, g_{1}^{k}, \ldots, g_{j_{k}}^{k}\right]\right]\right):= \\
{\left[i_{\gamma\left(w, v^{1}, v^{2}, \ldots, v^{k}\right)}, g_{1}^{1}, \ldots, g_{j_{k}}^{k}\right] .}
\end{gathered}
$$

These assignments make $\mu_{X}: C^{2} X \rightarrow C X$ into a well defined functor because of the equivariances of $\gamma$. These natural transformations commute appropriately to make $C$ into a monad on the category $\mathrm{Cat}_{0}$.

Theorem 5.11. The category of $C$-algebras is equivalent to the category of $T$ algebras.

Proof: $\quad$ Let $\mathcal{C}_{C}$ and $\mathcal{C}_{T}$ denote the categories of $C$-algebras and $T$-algebras respectively. We construct a functor $\phi: \mathcal{C}_{T} \rightarrow \mathcal{C}_{C}$. Let $(X, \Phi)$ be a $T$-algebra. Then $\Phi_{n}: T(n) \rightarrow$ Functors $\left(X^{n}, X\right)$ is a sequence of maps that is natural in $n$, preserves identity $1 \in T(1)$, and preserves compositions $\gamma$. This sequence of maps completely describes the algebraic structure. Let $h^{\prime}$ denote the element of Functors $\left(\bigcup_{n \geq 0}\left(T(n) \times X^{n}\right), X\right)$ that corresponds to the sequence under the bijection

$$
\text { Functors }\left(\bigcup_{n \geq 0}\left(T(n) \times X^{n}\right), X\right) \leftrightarrow \prod_{n \geq 0} \text { Functors }\left(T(n), X^{X^{n}}\right) .
$$

Then

$$
\begin{aligned}
& h^{\prime}\left(w_{f}, x_{1}, \ldots, x_{n}\right)=h^{\prime}\left(w, x_{f 1}, \ldots, x_{f m}\right) \\
& h^{\prime}\left(i_{w_{f}}, g_{1}, \ldots, g_{n}\right)=h^{\prime}\left(i_{w}, g_{f 1}, \ldots, g_{f m}\right)
\end{aligned}
$$

because

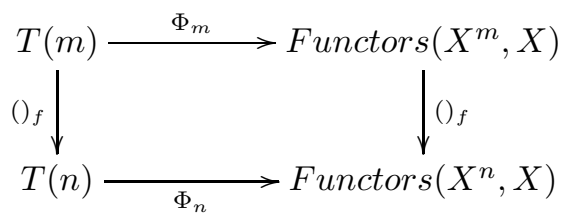

commutes. Hence $h^{\prime}: \bigcup_{n \geq 0}\left(T(n) \times X^{n}\right) \rightarrow X$ induces a functor $h: C X \rightarrow X$, namely

$$
\begin{gathered}
{\left[w, x_{1}, \ldots, x_{m}\right] \mapsto \Phi_{m}(w)\left(x_{1}, \ldots x_{m}\right)} \\
{\left[i_{w}, g_{1}, \ldots, g_{m}\right] \mapsto \Phi_{m}\left(i_{w}\right)\left(y_{1}, \ldots, y_{m}\right) \circ \Phi_{m}(w)\left(g_{1}, \ldots, g_{m}\right)} \\
=\Phi_{m}(w)\left(g_{1}, \ldots, g_{m}\right)
\end{gathered}
$$

for $g_{i}: x_{i} \rightarrow y_{i}$. Then $h: C X \rightarrow X$ makes $X$ into a $C$-algebra because the diagrams
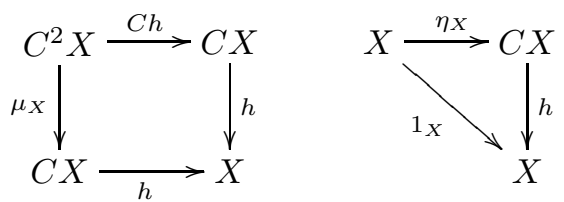
commute.

Define $\phi((X, \Phi)):=(X, h)$. For a morphism $f:(X, \Phi) \rightarrow(Y, \Psi)$ of $T$-algebras, let $\phi(f): X \rightarrow Y$ be the same functor as $f$ on the underlying categories. Then

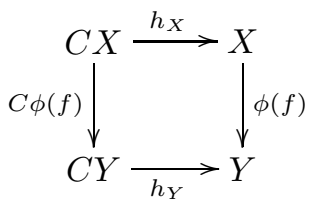

commutes. Then $\phi: \mathcal{C}_{T} \rightarrow \mathcal{C}_{C}$ is obviously a functor.

An "inverse" to $\phi$ can easily be constructed using the bijection ([6). For example, let $(X, h)$ be a $C$-algebra. Then $h: C X \rightarrow X$ corresponds uniquely to a functor $h^{\prime}: \bigcup_{n \geq 0}\left(T(n) \times X^{n}\right) \rightarrow X$ which satisfies

$$
\begin{aligned}
& h^{\prime}\left(w_{f}, x_{1}, \ldots, x_{n}\right)=h^{\prime}\left(w, x_{f 1}, \ldots, x_{f m}\right) \\
& h^{\prime}\left(i_{w_{f}}, g_{1}, \ldots, g_{n}\right)=h^{\prime}\left(w, g_{f 1}, \ldots, g_{f m}\right)
\end{aligned}
$$

and $h^{\prime}$ corresponds uniquely to some sequence $\Phi_{n}$ natural in $n$ which preserves 1 and $\gamma$.

The equivalence of Theorem 5.10 yields the desired result.

The concept of theory can be generalized to handle algebraic structures on more than one set, such as modules.

Definition 5.6. A theory on a set of objects $J$, also called a many-sorted theory, is a category $\mathbf{T}$ whose objects are finite sequences $\left(j_{1}^{m_{1}}, \ldots, j_{p}^{m_{p}}\right)$ with $j_{1}, \ldots, j_{p} \in$ $J, p \geq 1$, and $m_{1}, \ldots, m_{p} \in \mathbb{N}_{0}$ such that $\left(j_{1}^{m_{1}}, \ldots, j_{p}^{m_{p}}\right)$ is a product of copies of $j \in J$ where each $j$ appears $\sum_{r: j_{r}=j} m_{r}$ times. Each sequence is equipped with a limiting cone. Objects are equal to their reduced form, e.g. $\left(j^{m_{1}}, j^{m_{2}}\right)=\left(j^{m_{1}+m_{2}}\right)$. We also abbreviate $\left(j^{1}\right)=j$.

Example 12. An ordinary theory is a theory on one object, i.e. on the set $\{1\}$. We previously used $n$ to denote $1^{n}$ in the new notation.

Example 13. Let $X_{1}$ and $X_{2}$ be categories. Then the endomorphism theory

$\operatorname{End}\left(X_{j}: j \in J\right)$ on $X_{1}$ and $X_{2}$ is an example of a theory on the set $J=\{1,2\}$. The morphisms are

$$
\begin{gathered}
\operatorname{Mor}_{E n d\left(X_{j}: j \in J\right)}\left(\left(j_{1}^{m_{1}}, \ldots, j_{p}^{m_{p}}\right),\left(k_{1}^{n_{1}}, \ldots, k_{q}^{n_{q}}\right)\right):= \\
\text { Functors }\left(X_{j_{1}}^{m_{1}} \times \cdots \times X_{j_{p}}^{m^{p}}, X_{k_{1}}^{n_{1}} \times \cdots \times X_{k_{q}}^{n^{q}}\right)
\end{gathered}
$$

for $j_{r}, k_{s} \in\{1,2\}$ and $m_{r}, n_{s} \in \mathbb{N}_{0}$. One easily sees that $1^{0}$ and $2^{0}$ as well as $\left(1^{0}, 2^{0}\right)$ and $\left(2^{0}, 1^{0}\right)$ are terminal objects and that $\left(j_{1}^{m_{1}}, \ldots, j_{p}^{m_{p}}\right)$ is a product of $\sum_{r: j_{r}=1} m_{r}$ copies of 1 and $\sum_{r: j_{r}=2} m_{r}$ copies of 2 equipped with the usual projections. Note also that there is bijective correspondence.

$$
\begin{gathered}
\operatorname{Mor}_{\operatorname{End}\left(X_{j}: j \in J\right)}\left(\left(j_{1}^{m_{1}}, \ldots, j_{p}^{m_{p}}\right),\left(k_{1}^{n_{1}}, \ldots, k_{q}^{n_{q}}\right)\right) \leftrightarrow \\
\prod_{r: k_{r}=1} \operatorname{Mor}_{\operatorname{End}\left(X_{j}: j \in J\right)}\left(\left(j_{1}^{m_{1}}, \ldots, j_{p}^{m_{p}}\right), 1\right)^{\times n_{r}} \times \prod_{s: k_{s}=2} \operatorname{Mor}_{\operatorname{End}\left(X_{j}: j \in J\right)}\left(\left(j_{1}^{m_{1}}, \ldots, j_{p}^{m_{p}}\right), 2\right)^{\times n_{s}}
\end{gathered}
$$

In other words, the theory is determined by the sets

$$
\operatorname{Mor}_{\operatorname{End}\left(X_{j}: j \in J\right)}\left(\left(j_{1}^{m_{1}}, \ldots, j_{p}^{m_{p}}\right), 1\right)=: \operatorname{End}\left(X_{j}: j \in J\right)_{1}\left(j_{1}^{m_{1}}, \ldots, j_{p}^{m_{p}}\right)
$$




$$
\operatorname{Mor}_{\operatorname{End}\left(X_{j}: j \in J\right)}\left(\left(j_{1}^{m_{1}}, \ldots, j_{p}^{m_{p}}\right), 2\right)=: \operatorname{End}\left(X_{j}: j \in J\right)_{2}\left(j_{1}^{m_{1}}, \ldots, j_{p}^{m_{p}}\right)
$$

where $j_{1}, \ldots, j_{p} \in\{1,2\}$ and $m_{1}, \ldots, m_{p} \in \mathbb{N}_{0}$ such that $j_{r} \neq j_{r+1}$ for all $1 \leq r \leq$ $p-1$.

Note also that for $n_{1}, \ldots, n_{q} \in \mathbf{N}_{0}$ and $k_{1}, \ldots, k_{q} \in J$ and maps

$$
\begin{aligned}
& f: \sum_{r: j_{r}=1} m_{r} \rightarrow \sum_{r: k_{r}=1} n_{r} \\
& g: \sum_{s: j_{s}=2} m_{s} \rightarrow \sum_{s: k_{s}=2} n_{s}
\end{aligned}
$$

in $\Gamma$ we have substitution maps

$$
\begin{aligned}
& \operatorname{End}\left(X_{j}: j \in J\right)_{1}\left(j_{1}^{m_{1}}, \ldots, j_{p}^{m_{p}}\right) \stackrel{()_{f, g}}{\longrightarrow} \operatorname{End}\left(X_{j}: j \in J\right)_{1}\left(k_{1}^{n_{1}}, \ldots, k_{q}^{n_{q}}\right) \\
& \operatorname{End}\left(X_{j}: j \in J\right)_{2}\left(j_{1}^{m_{1}}, \ldots, j_{p}^{m_{p}}\right) \stackrel{()_{f, g}}{\longrightarrow} \operatorname{End}\left(X_{j}: j \in J\right)_{2}\left(k_{1}^{n_{1}}, \ldots, k_{q}^{n_{q}}\right) .
\end{aligned}
$$

For example, let $w \in \operatorname{End}\left(X_{j}: j \in J\right)_{1}\left(1^{2}, 2^{2}, 1^{1}, 2^{2}\right)$ and

$$
f:=\left(\begin{array}{ccc}
1 & 2 & 3 \\
1 & 1 & 1
\end{array}\right), \quad g:=\left(\begin{array}{cccc}
1 & 2 & 3 & 4 \\
1 & 2 & 2 & 1
\end{array}\right)
$$

where $\left(k_{1}^{n_{1}}, k_{2}^{n_{2}}\right)=\left(1^{1}, 2^{2}\right)$ so that

$$
f: 3 \rightarrow 1, \quad g: 4 \rightarrow 2 .
$$

Then $w_{f, g} \in \operatorname{End}\left(X_{j}: j \in J\right)_{1}\left(1^{1}, 2^{2}\right)$ is defined by

$$
\begin{aligned}
w_{f, g}\left(x_{1}^{1}, x_{1}^{2}, x_{2}^{2}\right) & :=w\left(x_{f 1}^{1}, x_{f 2}^{1}, x_{g 1}^{2}, x_{g 2}^{2}, x_{f 3}^{1}, x_{g 3}^{2}, x_{g 4}^{2}\right) \\
& =w\left(x_{1}^{1}, x_{1}^{1}, x_{1}^{2}, x_{2}^{2}, x_{1}^{1}, x_{2}^{2}, x_{1}^{2}\right) .
\end{aligned}
$$

The notation ()$_{f, g}$ suppresses the dependence of ()$_{f, g}$ on $\left(j_{1}^{m_{1}}, \ldots, j_{p}^{m_{p}}\right)$ and $\left(k_{1}^{n_{1}}, \ldots, k_{q}^{n_{q}}\right)$.

There also two compositions $\gamma_{1}$ and $\gamma_{2}$. For example

$$
\gamma_{1}: \operatorname{End}\left(X_{j}: j \in J\right)_{1}\left(1^{2}, 2^{2}\right) \times \operatorname{End}\left(X_{j}: j \in J\right)_{1}\left(\bar{n}_{1}\right) \times \operatorname{End}\left(X_{j}: j \in J\right)_{1}\left(\bar{n}_{2}\right) \times
$$$$
\times \operatorname{End}\left(X_{j}: j \in J\right)_{2}\left(\bar{n}_{3}\right) \times \operatorname{End}\left(X_{j}: j \in J\right)_{2}\left(\bar{n}_{4}\right) \rightarrow \operatorname{End}\left(X_{j}: j \in J\right)_{1}\left(\bar{n}_{1} \cdot \bar{n}_{2} \cdot \bar{n}_{3} \cdot \bar{n}_{4}\right)
$$

and

$$
\begin{aligned}
& \gamma_{2}: \operatorname{End}\left(X_{j}: j \in J\right)_{2}\left(2^{3}, 1^{1}\right) \times \operatorname{End}\left(X_{j}: j \in J\right)_{2}\left(\bar{n}_{1}\right) \times \operatorname{End}\left(X_{j}: j \in J\right)_{2}\left(\bar{n}_{2}\right) \times \\
& \times \operatorname{End}\left(X_{j}: j \in J\right)_{2}\left(\bar{n}_{3}\right) \times \operatorname{End}\left(X_{j}: j \in J\right)_{1}\left(\bar{n}_{4}\right) \rightarrow \operatorname{End}\left(X_{j}: j \in J\right)_{2}\left(\bar{n}_{1} \cdot \bar{n}_{2} \cdot \bar{n}_{3} \cdot \bar{n}_{4}\right)
\end{aligned}
$$
where $\bar{n}_{1} \cdot \bar{n}_{2} \cdot \bar{n}_{3} \cdot \bar{n}_{4}$ means to concatenate the objects $\bar{n}_{1}, \ldots, \bar{n}_{4}$ and to reduce, e.g. $\left(1^{1}, 2^{2}\right) \cdot\left(2^{3}, 1^{2}\right)=\left(1^{1}, 2^{5}, 1^{2}\right)$.

There are also units $1_{1} \in \operatorname{End}\left(X_{j}: j \in J\right)_{1}(1)$ and $1_{2} \in \operatorname{End}\left(X_{j}: j \in J\right)_{2}(2)$.

The compositions are associative, unital, and equivariant. The substitution is also functorial. This example easily extends to arbitrary $J$.

Definition 5.7. Let $\Gamma_{J}$ denote the category whose objects are finite sequences $\left(j_{1}^{m_{1}}, \ldots, j_{p}^{m_{p}}\right)$ with $j_{1}, \ldots, j_{p} \in J, p \geq 1$, and $m_{1}, \ldots, m_{p} \in \mathbb{N}_{0}$. Objects are equal to their reduced form, e.g. $\left(j^{m_{1}}, j^{m_{2}}\right)=\left(j^{m_{1}+m_{2}}\right)$. We also abbreviate $\left(j^{1}\right)=j$. The morphisms are

$$
\operatorname{Mor}_{\Gamma_{J}}\left(\left(j_{1}^{m_{1}}, \ldots, j_{p}^{m_{p}}\right),\left(k_{1}^{n_{1}}, \ldots, k_{q}^{n_{q}}\right)\right):=\prod_{\ell \in J} \operatorname{Mor}_{\Gamma}\left(\sum_{r: j_{r}=\ell} m_{r}, \sum_{s: k_{s}=\ell} n_{s}\right)
$$

where $\Gamma$ denotes the category in Definition 5.2 . 
In this definition the hom sets are assumed to be disjoint.

Several of the results on theories carry over to these generalized theories on a set of objects.

Theorem 5.12. A theory $\mathbf{T}$ on a set of objects $J$ is equivalent to a collection of functors $\left\{\mathbf{T}_{j}: \Gamma_{J} \rightarrow\right.$ Sets $\left.\mid j \in J\right\}$ equipped with compositions

$$
\begin{gathered}
\gamma_{j}: \mathbf{T}_{j}\left(j_{1}^{k_{1}}, \ldots, j_{p}^{k_{p}}\right) \times \mathbf{T}_{j_{1}}\left(\bar{n}_{1}^{1}\right) \times \cdots \times \mathbf{T}_{j_{1}}\left(\bar{n}_{k_{1}}^{1}\right) \times \\
\times \mathbf{T}_{j_{2}}\left(\bar{n}_{1}^{2}\right) \times \cdots \times \mathbf{T}_{j_{2}}\left(\bar{n}_{k_{2}}^{2}\right) \times \\
\cdots \\
\times \mathbf{T}_{j_{p}}\left(\bar{n}_{1}^{p}\right) \times \cdots \times \mathbf{T}_{j_{p}}\left(\bar{n}_{k_{p}}^{p}\right) \rightarrow \mathbf{T}_{j}\left(\bar{n}_{1}^{1} \cdots \bar{n}_{k_{1}}^{1} \cdot \bar{n}_{1}^{2} \cdots \bar{n}_{k_{2}}^{2} \cdots \bar{n}_{1}^{p} \cdots \bar{n}_{k_{p}}^{p}\right)
\end{gathered}
$$

for each $j \in J$ and $\left(j_{1}^{k_{1}}, \ldots, j_{p}^{k_{p}}\right), \bar{n}_{1}^{1}, \ldots, \bar{n}_{k_{p}}^{p} \in O b j \Gamma_{J}$ and equipped with units $1_{j} \in \mathbf{T}_{j}(j)$ for each $j \in J$ which satisfy analogues of (1) through (5) in Lemma 5.2 Elements of $\mathbf{T}_{j}(\bar{n})$ are called words.

Proof: $\quad$ Set $\mathbf{T}_{j}(\bar{n}):=\operatorname{Mor}_{\mathbf{T}}(\bar{n}, j)$ and proceed like in the case of a theory on the set $\{1\}$.

Example 14. The theory $\mathbf{R}$ of theories is a theory on the set $\mathbb{N}_{0}$. There are three types of generating morphisms.

- For each $k \geq 1$ and $n_{1}, \ldots, n_{k} \geq 0$ there is a morphism $\gamma:\left(k, n_{1}, \ldots, n_{k}\right) \rightarrow$ $\left(n_{1}+\cdots+n_{k}\right)$ called composition

- For each $f: m \rightarrow n$ in $\Gamma$ there is a morphism ()$_{f}:(m) \rightarrow(n)$ called substitution

- There is a morphism $1:\left(1^{0}\right) \rightarrow\left(1^{1}\right)$ called the unit.

The substitution and unit are not to be confused with the substitution and units with which every theory on a set of objects is equipped. These morphisms must satisfy the relations of theories in Lemma [5.2 namely associativity, equivariances, unitality, and functoriality.

Next one can speak of morphisms of theories on the set $J$ as well as algebras for theories on the set $J$ just as in the case $J=\{1\}$.

Definition 5.8. A morphism of theories on a set $J$ is a functor $\Phi: \mathbf{S} \rightarrow \mathbf{T}$ such that $\Phi\left(j_{1}^{m_{1}}, \ldots, j_{p}^{m_{p}}\right)=\left(j_{1}^{m_{1}}, \ldots, j_{p}^{m_{p}}\right)$ and $\Phi(p r)=p r$ for every projection.

Theorem 5.13. The analogue of Theorem 5.10 holds for theories on a set of objects $J$.

Definition 5.9. Let $\mathbf{T}$ be a theory on the set $J$ and $\left\{X_{j} \mid j \in J\right\}$ a collection of categories. Then $\left\{X_{j}\right\}_{j}$ form an algebra over $\mathbf{T}$ or a $\mathbf{T}$-algebra if they are equipped with a morphism $\Phi: \mathbf{T} \rightarrow \operatorname{End}\left(X_{j}: j \in J\right)$ of theories on $J$.

Example 15. Let $\mathbf{R}$ denote the theory of theories. Let $T$ be a theory. Then $\left\{T(j) \mid j \in \mathbb{N}_{0}\right\}$ form an $\mathbf{R}$-algebra. In other words, a theory is an algebra for the theory of theories. A morphism of theories is nothing more than a morphism of algebras over the theory of theories.

One can use the theory $\mathbf{R}$ of theories to construct a monad $C$ on the category $\prod_{n \geq 0}$ Sets whose algebras are the usual theories. In fact, $C T$ is the sequence of sets underlying the free theory on $T$. This free theory is essential to several of the 
proofs in this paper. Let $T=(T(n))_{n \geq 0}$ be an object of $\prod_{n \geq 0}$ Sets and $J:=\mathbb{N}_{0}$. Then the free theory on $T$ is defined by

$$
C T(n):=\frac{\bigcup_{\bar{m} \in O b j \Gamma_{J}} \mathbf{R}_{n}(\bar{m}) \times T\left(j_{1}\right)^{\times m_{1}} \times \cdots \times T\left(j_{p}\right)^{\times m_{p}}}{\Gamma_{J}}
$$

where $\bar{m}=\left(j_{1}^{m_{1}}, \ldots, j_{p}^{m_{p}}\right)$.

One can generalize the notion of theory in yet another direction. Instead of considering arbitrary sets $J$, one can consider theories which are also 2-categories in which every 2-cell is iso. These will be used to describe pseudo algebras in a compact way. See 43] for a more general concept of enriched Lawvere theory.

Definition 5.10. A theory enriched in groupoids is a 2-category $\mathcal{T}$ with iso 2-cells and with objects $0,1,2, \ldots$ such that $n$ is the 2-product of 1 with itself $n$ times in the 2-category $\mathcal{T}$ and each $n$ is equipped with a limiting 2-cone.

This definition means for each $n \in O b j \mathcal{T}$ we have chosen morphisms $\pi_{i}^{n}=p r_{i}$ : $n \rightarrow 1$ for $i=1, \ldots, n$ with the universal property that

$$
\operatorname{Mor}_{\mathcal{T}}(m, n) \stackrel{\pi^{n} \circ}{\longrightarrow} 2-\operatorname{Cone}(m, F)
$$

is an isomorphism for all $m \in \operatorname{Obj} \mathcal{T}$, where $F:\{1, \ldots, n\} \rightarrow \mathcal{T}$ is the 2-functor which is constant 1 . It is tempting to call such a theory a 2 -theory, but we reserve that name for something else. As before, we use the notation $\mathcal{T}(n)$ for the category $\operatorname{Mor}_{\mathcal{T}}(n, 1)$. Using the universal property, we can construct $\prod$ and $(\ldots)$ for the 2cells. For any object $m \in O b j T$, morphisms $w_{i}, v_{i}: m \rightarrow 1$, and 2-cells $\alpha_{i}: w_{i} \Rightarrow v_{i}$ for $i=1, \ldots, n$ there exists a unique 2 -cell $\prod_{j=1}^{n} \alpha_{j}: \prod_{j=1}^{n} w_{j} \Rightarrow \prod_{j=1}^{n} v_{j}$ such that

$$
i_{p r_{i}} * \prod_{j=1}^{n} \alpha_{j}=\alpha_{i}
$$

for all $i=1, \ldots n$. For any $k \in \mathbb{N}_{0}$, any morphisms $w_{i}, v_{i}: n_{i} \rightarrow 1$, and any 2-cells $\alpha_{i}: w_{i} \Rightarrow v_{i}$ for $i=1, \ldots, k$ there is a unique 2 -cell $\left(\alpha_{1}, \ldots, \alpha_{k}\right):\left(w_{1}, \ldots, w_{k}\right) \Rightarrow$ $\left(v_{1}, \ldots, v_{k}\right)$ such that

$$
i_{p r_{i}} *\left(\alpha_{1}, \ldots, \alpha_{k}\right)=\left(\alpha_{i}\right)_{\iota_{i}}
$$

for all $i=1, \ldots, k$.

Example 16. Let $X$ be a category. Then the endomorphism theory End $(X)$ enriched in groupoids has objects $0,1,2, \ldots$, morphisms $\operatorname{ObjMor}_{\operatorname{End}(X)}(m, n)=$ Functors $\left(X^{m}, X^{n}\right)$ and 2-cells the natural isomorphisms.

Most of the work on theories carries over to the enriched context with minor additions for the 2-cells. The statements of the relevant theorems are as follows. The term map is simply replaced by functor.

Lemma 5.14. Let $\mathcal{T}$ be a theory enriched in groupoids. Then the category $\operatorname{Mor}_{\mathcal{T}}(m, n)$ is isomorphic to the product category $\prod_{j=1}^{n} \operatorname{Mor}_{\mathcal{T}}(m, 1)$.

Lemma 5.15. Let $\mathcal{T}$ be a theory enriched in groupoids. Then for all $k, n_{1}, \ldots, n_{k} \in$ $\{0,1, \ldots\}$ there is a functor $\gamma: \mathcal{T}(k) \times \mathcal{T}\left(n_{1}\right) \times \cdots \times \mathcal{T}\left(n_{k}\right) \rightarrow \mathcal{T}\left(n_{1}+\cdots+n_{k}\right)$ called composition and for every function $f:\{1, \ldots, k\} \rightarrow\{1, \ldots, \ell\}$ there is a functor $\mathcal{T}(k) \stackrel{()_{f}}{\longrightarrow} \mathcal{T}(\ell)$ called substitution. These functors satisfy the enriched analogues of (1) through (5) in Lemma 5.2. 
Proof: Define $\gamma\left(w, w_{1}, \ldots, w_{k}\right):=w \circ\left(w_{1}, \ldots, w_{k}\right)$ as before. Additionally, define $\gamma\left(\alpha, \alpha_{1}, \ldots, \alpha_{k}\right):=\alpha *\left(\alpha_{1}, \ldots, \alpha_{k}\right)$ for 2-cells. Define $w_{f}:=w \circ f^{\prime}$ as before and $\alpha_{f}:=\alpha * i_{f^{\prime}}$ where $i_{f^{\prime}}: f^{\prime} \Rightarrow f^{\prime}$ is the identity 2-cell of the morphism $f^{\prime}$ in $\mathcal{T}$ and $\alpha: w \Rightarrow v$ is a 2 -cell. The rest of proof is similar to Lemma 5.2

Lemma 5.16. Let $\mathcal{T}$ be a 2 -functor from $\Gamma$ to Cat equipped with functors $\gamma: \mathcal{T}(k) \times \mathcal{T}\left(n_{1}\right) \times \cdots \times \mathcal{T}\left(n_{k}\right) \rightarrow \mathcal{T}\left(n_{1}+\cdots+n_{k}\right)$ and an object $1 \in \mathcal{T}(1)$ which satisfy (1) through (5) of Lemma 5.2 where $\mathcal{T}(f)=:()_{f}$ for functions $f: k \rightarrow \ell$. Then $\mathcal{T}$ determines a theory with $\operatorname{Mor}(n, 1)=\mathcal{T}(n)$ for all $n \geq 0$.

Proof:

Theorem 5.17. A theory $\mathcal{T}$ enriched in groupoids is determined by either of the following equivalent collections of data:

(1) A 2-category $\mathcal{T}$ with objects $0,1,2, \ldots$ such that $n$ is the 2-categorical product of 1 with itself $n$ times and each $n$ is equipped with a limiting 2-cone

(2) A 2-functor $\mathcal{T}: \Gamma \rightarrow$ Cat equipped with functors $\gamma: \mathcal{T}(k) \times \mathcal{T}\left(n_{1}\right) \times \cdots \times$ $\mathcal{T}\left(n_{k}\right) \rightarrow \mathcal{T}\left(n_{1}+\cdots+n_{k}\right)$ and a unit $1 \in \mathcal{T}(1)$ which satisfy (1) through (5) of Lemma [5.2.

Proof: In each description $\operatorname{Mor}_{\mathcal{T}}(n, 1)$ is the same. By the universality of 2-products this determines the rest of the theory.

Definition 5.11. Let $\mathcal{S}$ and $\mathcal{T}$ be theories enriched over groupoids. In the 2categorical description of $\mathcal{S}$ and $\mathcal{T}$ a morphism of theories enriched over groupoids $\Phi: \mathcal{S} \rightarrow \mathcal{T}$ is a 2-functor from the 2-category $\mathcal{S}$ to the 2-category $\mathcal{T}$ such that $\Phi\left(n_{\mathcal{S}}\right)=n_{\mathcal{T}}$ and $\Phi\left(p r_{i}\right)=p r_{i}$ for all projections.

The analogue for Lemma 5.6 incorporates the 2-cells below.

Lemma 5.18. Let $\Phi: \mathcal{S} \rightarrow \mathcal{T}$ be a morphism of theories enriched over groupoids.

(1) Let $f:\{1, \ldots, k\} \rightarrow\{1, \ldots, \ell\}$ be a function. As usual, $f^{\prime}: \ell \rightarrow k$ denotes the unique morphism in any theory such that

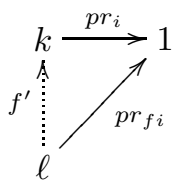

commutes. Then $\Phi\left(f^{\prime}\right)=f^{\prime}$.

(2) Let $f:\{1, \ldots, k\} \rightarrow\{1, \ldots, \ell\}$ be a function and $w \in M o r_{\mathcal{S}}(k, 1)$. Then $\Phi\left(w_{f}\right)=\Phi(w)_{f}$.

(3) Let $w_{j}, v_{j} \in \operatorname{Mor}_{\mathcal{S}}(m, 1)$ and $\alpha_{j}: w_{j} \Rightarrow v_{j}$ for $j=1, \ldots, n$. Then $\Phi\left(\prod_{j=1}^{n} w_{j}\right)=\prod_{j=1}^{n} \Phi\left(w_{j}\right)$ and $\Phi\left(\prod_{j=1}^{n} \alpha_{j}\right)=\prod_{j=1}^{n} \Phi\left(\alpha_{j}\right)$.

(4) Let $w_{j}, v_{j} \in \operatorname{Mor}_{\mathcal{S}}\left(n_{j}, 1\right)$ for $j=1, \ldots, k$. Then $\Phi\left(w_{1}, \ldots, w_{k}\right)=\left(\Phi\left(w_{1}\right), \ldots, \Phi\left(w_{k}\right)\right)$ and $\Phi\left(\alpha_{1}, \ldots, \alpha_{k}\right)=\left(\Phi\left(\alpha_{1}\right), \ldots, \Phi\left(\alpha_{k}\right)\right)$.

Theorem 5.19. Let $\mathcal{S}$ and $\mathcal{T}$ be theories enriched over groupoids. Then a morphism $\mathcal{S} \rightarrow \mathcal{T}$ of theories enriched over groupoids is given by either of the following equivalent collections of data:

(1) A 2-functor $\Phi: \mathcal{S} \rightarrow \mathcal{T}$ such that $\Phi\left(n_{\mathcal{S}}\right)=n_{\mathcal{T}}$ for all $n_{\mathcal{S}} \in O b j \mathcal{S}$ and $\Phi\left(p r_{i}\right)=p r_{i}$ for all projections 
(2) A 2-natural transformation $\Phi: \mathcal{S} \Rightarrow \mathcal{T}$ of the 2-functors $\mathcal{S}, \mathcal{T}: \Gamma \rightarrow C a t$ which preserves the $\gamma$ 's and the units.

Theorem 5.20. The 2-category of theories enriched over groupoids with objects and morphisms as in (1) of Theorems 5.17 and 5.19 is 2-equivalent to the 2-category with objects and morphisms as in (2) of Theorems 5.17 and 5.19 .

We can now define algebras over theories enriched in groupoids in analogy to algebras over theories.

Definition 5.12. Let $X$ be a category and $\mathcal{T}$ a theory enriched over groupoids. Then $X$ is a $\mathcal{T}$-algebra if it is equipped with a morphism of theories $\mathcal{T} \rightarrow \operatorname{End}(X)$ enriched over groupoids. We also say $X$ is an algebra over the theory $\mathcal{T}$.

Our main example, pseudo $T$-algebras, will be given in the next section as strict $\mathcal{T}$-algebras, where $\mathcal{T}$ is obtained from the free theory on $T$.

\section{Pseudo T-Algebras}

In this section we introduce the 2-category of pseudo $T$-algebras for a theory T. A pseudo algebra in this paper is the same thing as a lax algebra in [24, 25], and [26. We construct from $T$ a theory $\mathcal{T}$ enriched over groupoids and show that a pseudo algebra over $T$ is the same thing as an algebra over $\mathcal{T}$. Theorem 6.6 says that the 2-category of pseudo $T$-algebras is 2-equivalent to the 2-category of strict $C$-algebras with pseudo morphisms for the 2-monad $C$ defined below. This 2category obtained from a 2-monad $C$ admits pseudo limits by a result of Blackwell, Kelly, and Power. Hence the 2-category of pseudo $T$-algebras admits pseudo limits. In the next section we give a concrete construction of the pseudo limit. For more on pseudo algebras over 2-monads see [23, 32], and 33.

Definition 6.1. Let $T$ be a theory. Let $X$ be a category. $X$ is a pseudo $T$ algebra or a pseudo algebra over $T$ if for every $n \in \mathbb{N}$ there is a map $\Phi_{n}: T(n) \rightarrow$ Functors $\left(X^{n}, X\right)$ (sometimes called the structure map) which is equipped with the following coherence isomorphisms and coherence diagrams between them. I write simply $\Phi$ for all $\Phi_{n}$. The coherence isomorphisms are indexed by the operations of theories and are as follows:

(1) For every $k \in \mathbb{N}, w \in T(k)$, and all words $w_{1}, \ldots, w_{k}$ there is a natural isomorphism $c_{w, w_{1}, \ldots, w_{k}}: \Phi\left(\gamma\left(w, w_{1}, \ldots, w_{k}\right)\right) \Rightarrow \gamma\left(\Phi(w), \Phi\left(w_{1}\right), \ldots, \Phi\left(w_{k}\right)\right)$. This means that composition is preserved by $\Phi$ up to natural isomorphism.

(2) There is a natural isomorphism $I: \Phi(1) \Rightarrow 1_{X}$ where 1 is the identity word and $1_{X}$ is the identity functor $X \rightarrow X$. This means that the action of the identity word is trivial up to a natural isomorphism.

(3) For every word $w \in T(m)$ and function $f:\{1, \ldots, m\} \rightarrow\{1, \ldots, n\}$ there is a natural isomorphism $s_{w, f}: \Phi\left(w_{f}\right) \Rightarrow \Phi(w)_{f}$ where the substituted functor $\Phi(w)_{f}: X^{n} \rightarrow X$ is defined in Example 8 In other words the equivariance is preserved by up to natural isomorphism.

The coherence diagrams are as follows. The commutivity of these diagrams means that they commute when evaluated on every tuple of objects of $X$ of appropriate length. Coherence diagrams are indexed by the relations of theories. 
(1) The action of $T$ on $X$ via $\Phi$ is associative up to the natural isomorphisms above. For example, for $u, v, w \in T(1)$

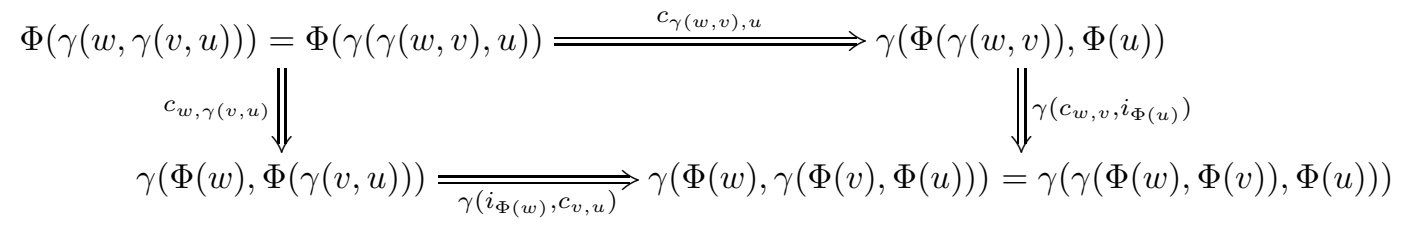

must commute where $i_{F}$ means the identity natural transformation $F \rightarrow F$ for a functor $F$.

(2) The natural isomorphism for the identity word commutes with the natural isomorphism for the composition, i.e. for every $n \in \mathbb{N}$ and every word $w \in T(n)$

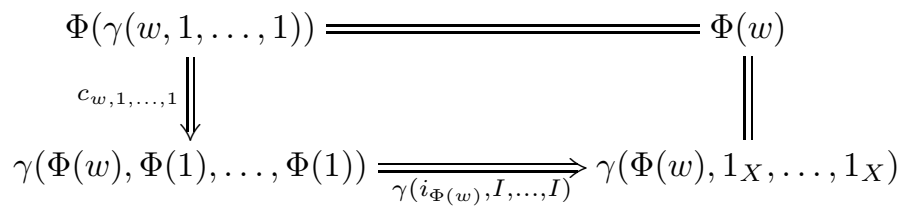

must commute where $1_{X}$ is the identity functor on $X$.

(3) The natural isomorphism for the identity word commutes with the natural isomorphism for the composition also in the sense that for every word $w \in T(n)$ the diagram

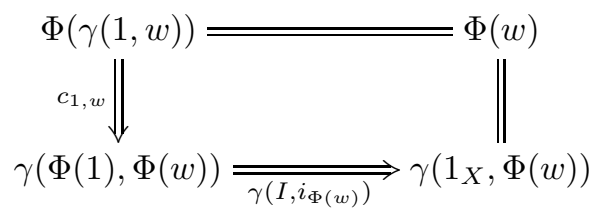

must commute.

(4) Let $f:\{1, \ldots, k\} \rightarrow\{1, \ldots, \ell\}$ be a function and let $\bar{f}:\left\{1,2, \ldots, n_{f 1}+n_{f 2}+\cdots+n_{f k}\right\} \rightarrow\left\{1,2, \ldots, n_{1}+n_{2}+\cdots+n_{\ell}\right\}$ be the function that moves entire blocks according to $f$. Then equivariance is preserved in the sense that the diagram

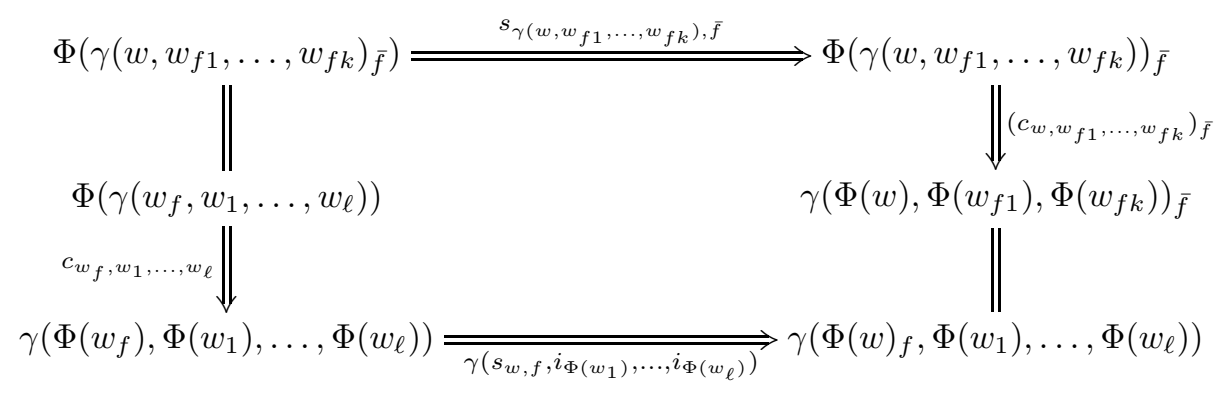

must commute.

(5) Let $g_{i}:\left\{1, \ldots, n_{i}\right\} \rightarrow\left\{1, \ldots, n_{i}^{\prime}\right\}$ be functions and let $g_{1}+\cdots+g_{k}:\left\{1,2, \ldots, n_{1}+\cdots+n_{k}\right\} \rightarrow\left\{1,2, \ldots, n_{1}^{\prime}+\cdots+n_{k}^{\prime}\right\}$ be the function obtained by placing $g_{1}, \ldots, g_{k}$ next to each other from left to right. 
Then equivariance is preserved in the sense that the diagram

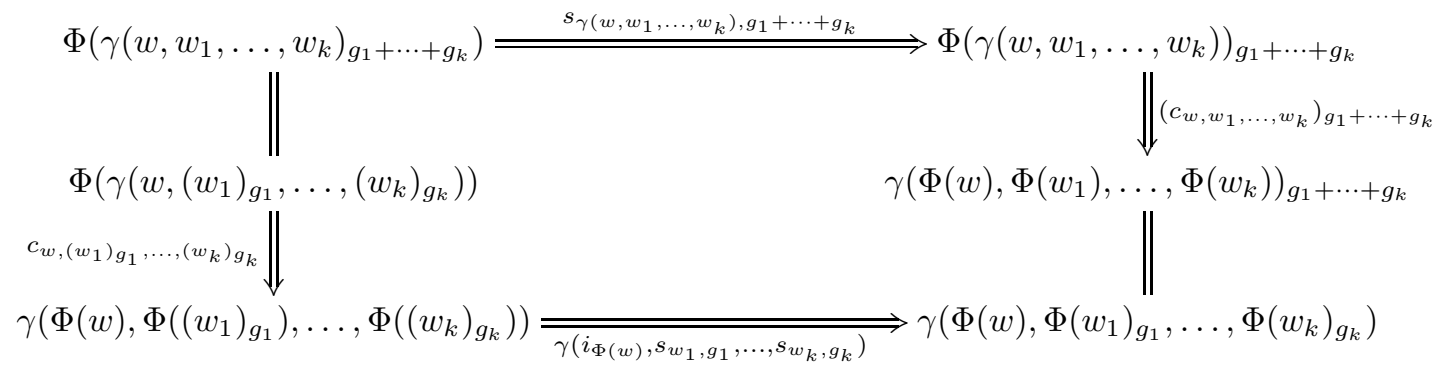

must commute.

(6) The substitution is associative up to the natural isomorphisms above, i.e. for every word $w \in T(\ell)$ and functions $f:\{1, \ldots, \ell\} \rightarrow\{1, \ldots, m\}$ and $g$ : $\{1, \ldots, m\} \rightarrow\{1, \ldots, n\}$ we mimic the equality $w_{g \circ f}=\left(w_{f}\right)_{g}$ by requiring the following diagram to commute.

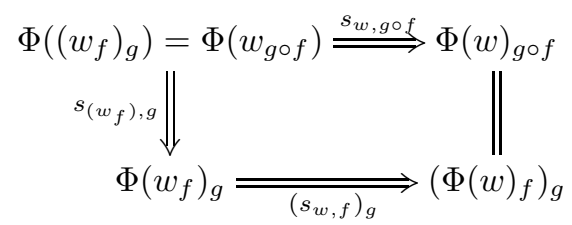

Here $\left(s_{w, f}\right)_{g}$ is the natural transformation which is defined for objects $A_{1}, \ldots, A_{n}$ of $X$ by $\left(s_{w, f}\right)_{g}\left(A_{1}, \ldots, A_{n}\right)=s_{w, f}\left(A_{g 1}, \ldots, A_{g m}\right)$.

(7) For all $w \in T(k)$ and $i d:\{1, \ldots, k\} \rightarrow\{1, \ldots, k\}$ the natural transformation $s_{w, i d}$ is the identity.

Remark 6.1. One can compactly describe the concept of a pseudo algebra as follows. A category $X$ is a pseudo $T$-algebra if it is equipped with a pseudo morphism of theories $\Phi: T \rightarrow \operatorname{End}(X)$. The assignment $\Phi$ is pseudo in the sense that the requirements of Lemma 5.7 are only satisfied up to coherence isos, namely the assignment preserves $\gamma$ up to $c$, preserves the identity up $I$, and is natural up to $s$ as in the diagrams below and these coherence isos satisfy coherence diagrams.

(1)
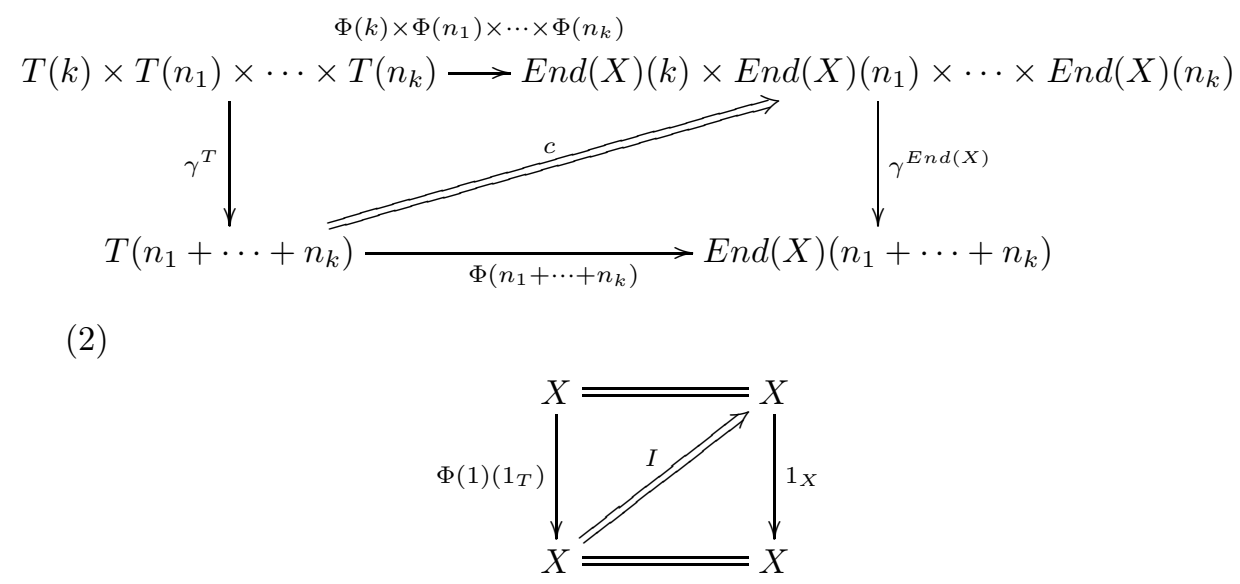
(3)

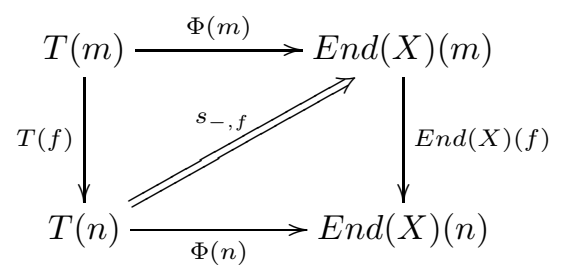

Remark 6.2. It is possible to describe the general form of these coherence diagrams. In general, a relation $\alpha \circ \beta=\alpha^{\prime} \circ \beta^{\prime}$ in the theory of theories and a tuple $\bar{w}$ of words gives rise to a coherence diagram

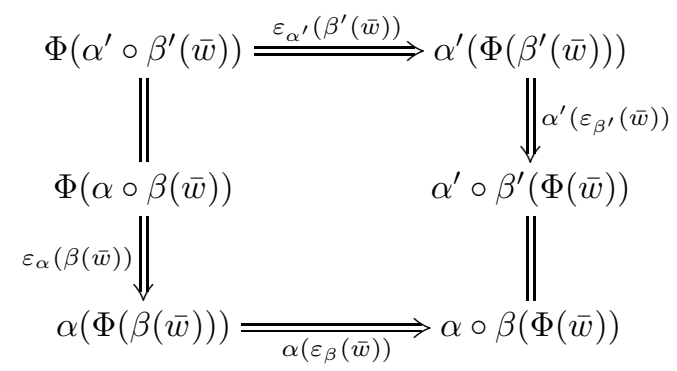

where $\varepsilon_{\alpha}, \varepsilon_{\alpha^{\prime}}, \varepsilon_{\beta}$, and $\varepsilon_{\beta^{\prime}}$ are the coherence isos associated to the morphisms $\alpha, \alpha^{\prime}, \beta, \beta^{\prime}$ respectively in the theory of theories and $\Phi(\bar{w})$ denotes the tuple of words obtained by applying $\Phi$ to each of the constituents of $\bar{w}$. Note that $\varepsilon_{\alpha}, \varepsilon_{\alpha^{\prime}}, \varepsilon_{\beta}$, and $\varepsilon_{\beta^{\prime}}$ are tuples of the 2-cells $c, I, s$ and identity 2-cells. In the definition of pseudo algebra above, the morphisms $\beta, \beta^{\prime}$ are tuples of generating morphisms in all cases except in (4). In (4) the $\beta^{\prime}$ is the result of applying a substitution morphism in the theory of theories to $\gamma$. This substitution morphism can be written in terms of $f$ appropriately. In this case we have $\varepsilon_{\beta^{\prime}}(\bar{w})=c_{w, w_{f 1}, \ldots, w_{f k}}$.

Definition 6.2. Let $X$ and $Y$ be pseudo $T$-algebras and $H: X \rightarrow Y$ a functor between the underlying categories. Denote the structure maps of $X$ and $Y$ by $\Phi$ and $\Psi$ respectively. For all $n \in \mathbb{N}$ and all $w \in T(n)$ let $\rho_{w}: H \circ \Phi(w) \Rightarrow \Psi(w) \circ(H, \ldots, H)$ be a natural isomorphism. Then $H$ is a pseudo morphism of pseudo T-algebras with coherence 2-cells $\rho_{w}$ (or just morphism of pseudo T-algebras for short) if the following coherence diagrams of natural isomorphisms are satisfied.

(1) For all $k \in \mathbb{N}, w \in T(k)$, and all words $w_{1}, \ldots, w_{k}$ of $T$

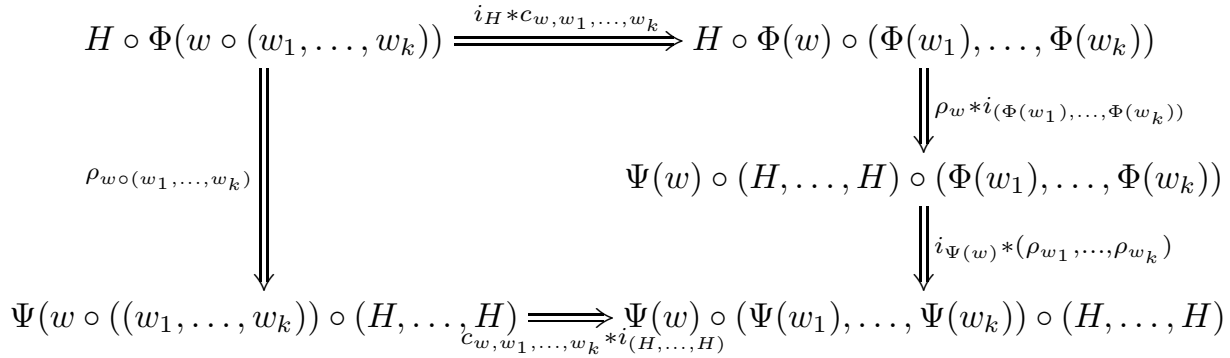

must commute. 
(2) The diagram

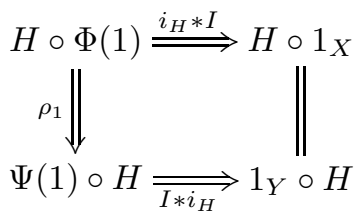

must commute.

(3) For every word $w \in T(m)$ and every function $f:\{1, \ldots, m\} \rightarrow\{1, \ldots, n\}$

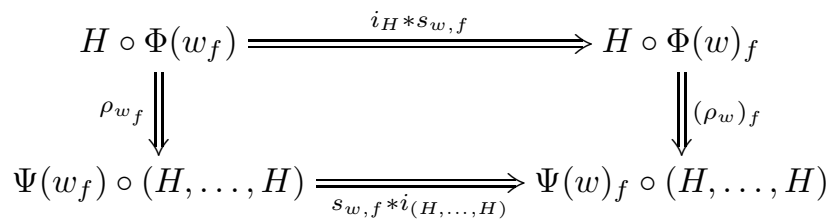

must commute.

Example 17. Let $T$ be the theory of commutative monoids and let FiniteSets be the category of finite sets. Define $A \coprod B:=A \times\{1\} \cup B \times\{2\}$ for finite sets $A$ and $B$. Define coproduct similarly for morphisms of finite sets. Then Ц FiniteSets $\times$ FiniteSets $\rightarrow$ FiniteSets is a functor which makes FiniteSets into a pseudo $T$-algebra, i.e. a pseudo commutative monoid. More generally, any symmetric monoidal category is a pseudo $T$-algebra.

Example 18. Let $T$ be the theory of commutative semi-rings. Then the category of finite dimensional complex vector spaces is a pseudo $T$-algebra whose structure is given by direct sum and tensor product. We also say this category is a pseudo commutative semi-ring.

Definition 6.3. Let $X, Y$, and $Z$ be pseudo $T$-algebras and $G: X \rightarrow Y, H: Y \rightarrow Z$ morphisms of pseudo $T$-algebras with coherence 2-cells $\rho_{w}^{G}$ and $\rho_{w}^{H}$ respectively. Then the composition $H \circ G$ is the composition of the underlying functors . It has the coherence 2-cells $\rho_{w}^{H \circ G}:=\left(\rho_{w}^{H} * i_{(G, \ldots, G)}\right) \odot\left(i_{H} * \rho_{w}^{G}\right): H \circ G \circ \Phi(w) \Rightarrow$ $\Psi(w) \circ(H \circ G, \ldots, H \circ G)$ where $\Phi(w)$ and $\Psi(w)$ denote the actions of $w$ on $X$ and $Z$ respectively.

Lemma 6.1. The composition of morphisms of pseudo T-algebras is a morphism of pseudo T-algebras.

Proof: $\quad$ Immediate.

Definition 6.4. Let $X$ and $Y$ be pseudo $T$-algebras and $G, H: X \rightarrow Y$ morphisms of pseudo $T$-algebras. Denote the action of the theory $T$ on $X$ and $Y$ by $\Phi$ and $\Psi$ respectively. A natural transformation $\alpha: G \Rightarrow H$ between the underlying functors is a 2-cell in the 2-category of pseudo T-algebras if for all $n \in \mathbb{N}$ and all $w \in T(n)$

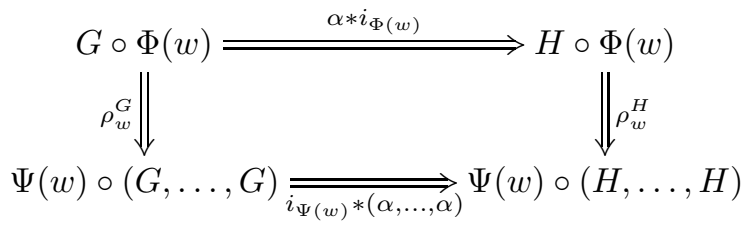


commutes. The vertical and horizontal compositions of the 2-cells are just the vertical and horizontal composition of the underlying natural transformations.

Lemma 6.2. The small pseudo T-algebras with morphisms and 2-cells defined above form a 2-category.

Proof: The axioms can be verified directly.

As mentioned in the last section, a pseudo $T$-algebra is the same thing as a strict $\mathcal{T}$-algebra. This was observed in [26]. One can see this as follows. Let $T^{\prime}$ denote the free theory on the sequence of sets underlying $T$. Recall that $T^{\prime}$ was described in terms of the sets $T^{\prime}(n)$ for $n \geq 0$ and the compositions, substitutions, and identities. From this description, the hom sets are $\operatorname{Mor}_{T^{\prime}}(m, n)=\prod_{j=1}^{n} T^{\prime}(m)$. There is a map of theories $T^{\prime} \rightarrow T$ which gives the theory structure on $T$. Let the underlying 1-category of the 2-category $\mathcal{T}$ be $T^{\prime}$. For $v, w \in \mathcal{T}(n)=\operatorname{Mor}_{\mathcal{T}}(n, 1)$ we define a unique iso 2-cell between $v$ and $w$ if $v$ and $w$ map to the same element of $T(n)$ under the map of theories $T^{\prime} \rightarrow T$. Otherwise there is no 2-cell between $v$ and $w$. With these definitions, the only 2 -cell between $w$ and $w$ is the identity and the vertical composition of 2-cells is uniquely defined. Thus $\mathcal{T}(n)$ is a category. Next define $\operatorname{Mor}_{\mathcal{T}}(m, n)$ to be the product category $\prod_{j=1}^{n} \mathcal{T}(m)$ for all $m, n \in \operatorname{Obj} \mathcal{T}$. From this it follows that there is a unique iso 2-cell between $v, w \in \operatorname{Mor}_{\mathcal{T}}(m, n)$ if they map to the same element of $\operatorname{Mor}_{T}(m, n)$ and otherwise there is no 2-cell. This uniquely defines the horizontal composition of 2-cells and $\mathcal{T}$ is a 2-category. From the definitions it also follows easily that $n$ is the 2-product of $n$ copies of 1 in $\mathcal{T}$. Hence $\mathcal{T}$ is a theory enriched in groupoids. In [26] $\mathcal{T}$ is denoted $(T h(T), G(T))$.

We introduce the notation $c, I, s$ for some of these 2-cells, which breaks the usual convention of labeling 2-cells by lowercase greek letters. Let

$$
c_{w, w_{1}, \ldots, w_{k}}:\left(()_{i d_{n_{1}+\cdots+n_{k}}}, \gamma\left(w, w_{1}, \ldots, w_{k}\right)\right) \Longrightarrow\left(\gamma, w, w_{1}, \ldots, w_{k}\right)
$$

denote the unique 2-cell for $w \in T(k), w_{i} \in T\left(n_{i}\right), i=1, \ldots, k$. The $\gamma$ on the right is a generator of the theory of theories while the $\gamma$ on the left is the composition in the theory $T$. The map $i d_{n_{1}+\cdots+n_{k}}$ is the identity of the object $n_{1}+\cdots+n_{k}$ in the category $\Gamma$. Let

$$
I:\left(()_{i d_{1}}, 1\right) \Longrightarrow(1, *)
$$

where $\left(()_{i d_{1}}, 1\right) \in \mathbf{R}_{1}(1) \times T(1)$ and $(1, *) \in \mathbf{R}_{1}\left(1^{0}\right) \times T(1)^{0}$. Let

$$
s_{w, f}:\left(()_{i d_{n}}, w_{f}\right) \Longrightarrow\left(()_{f}, w\right)
$$

denote the unique 2-cell for $w \in T(m)$ and $f: m \rightarrow n$ in $\Gamma$. We call these 2-cells as well as identity 2-cells the elementary 2-cells. By the following inductive proof, every other 2-cell in $\mathcal{T}$ can be obtained from these ones and their inverses.

Lemma 6.3. Let $\alpha$ be a word in the theory of theories, i.e. $\alpha \in \mathbf{R}_{n}(\bar{m})$ for some $n \in \mathbb{N}_{0}, \bar{m}=\left(j_{1}^{m_{1}}, \ldots, j_{p}^{m_{p}}\right)$, and $m:=m_{1}+\cdots+m_{p}$. Then the 2-cell

$$
\left(()_{i d_{n}}, \alpha\left(v_{1}, \ldots, v_{m}\right)\right) \Longrightarrow\left(\alpha, v_{1}, \ldots, v_{m}\right)
$$

in $\mathcal{T}$ can be expressed as a vertical composition

$$
\sigma_{s} \odot \sigma_{s-1} \odot \cdots \odot \sigma_{1}
$$

where each $\sigma_{r}$ is the result of applying a morphism in $\mathbf{R}$ to a tuple of elementary 2-cells. 
Proof: $\quad$ Let $\alpha=\alpha_{i} \circ \cdots \circ \alpha_{1}$ where $\alpha_{1}, \ldots, \alpha_{i}$ are tuples of generating morphisms in the theory $\mathbf{R}$ of theories such that $i$ is minimal. We induct on $i$. If $i=1$, then $\alpha$ is a generating morphism for $\mathbf{R}$ and the 2-cell

$$
\left(()_{i d_{n}}, \alpha\left(v_{1}, \ldots, v_{m}\right)\right) \Longrightarrow\left(\alpha, v_{1}, \ldots, v_{m}\right)
$$

must be one of $c, I$, or $s$. Now let $i \geq 1$ and suppose the Lemma holds for all words that can be expressed with $i$ terms or less. Suppose $\alpha \in \mathbf{R}_{n}(\bar{m})$ has an expression with $i+1$ terms but not does not have an expression with fewer terms. Then $\alpha=\beta \circ\left(\beta_{1}, \ldots, \beta_{k}\right)$ where $\beta$ is a generating morphism for the theory of theories and $\beta_{1}, \ldots, \beta_{k}$ are some words in the theory of theories, each with $i_{1}, \ldots, i_{k} \leq i$. Then the 2-cells

$$
\begin{aligned}
& \varepsilon_{1}:\left(()_{i d}, \beta_{1}\left(v_{1}, \ldots\right)\right) \Longrightarrow\left(\beta_{1}, v_{1}, \ldots\right) \\
& \varepsilon_{2}:\left(()_{i d}, \beta_{2}(\ldots)\right) \Longrightarrow\left(\beta_{2}, \ldots\right) \\
& \vdots \\
& \varepsilon_{k}:\left(()_{i d}, \beta_{k}\left(\ldots, v_{m}\right)\right) \Longrightarrow\left(\beta_{k}, \ldots, v_{m}\right)
\end{aligned}
$$

can be obtained from elementary 2-cells in the prescribed manner by the induction hypothesis. Here $i d$ is generically used to denote any identity morphism in $\Gamma$. Then

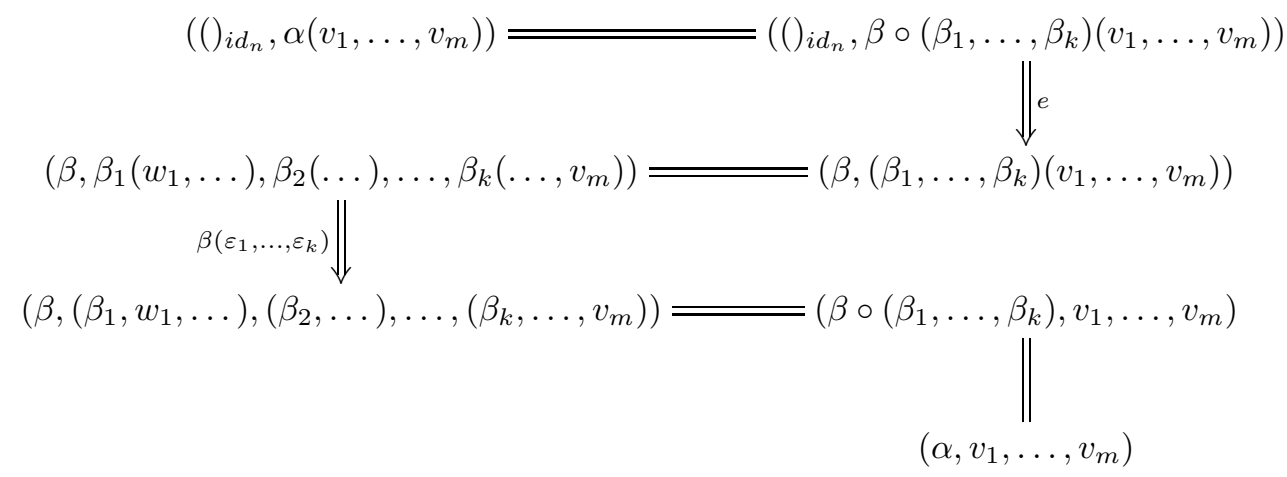

is also a composition of the prescribed type, where $e$ is an elementary 2-cell.

Lemma 6.4. Let $\alpha$ and $\beta$ be words in the theory of theories. Suppose that there is a 2-cell

$$
\left(\alpha, v_{1}, \ldots, v_{m_{1}}\right) \Longrightarrow\left(\beta, w_{1}, \ldots, w_{m_{2}}\right)
$$

in $\mathcal{T}$. Then this 2-cell is a vertical composition of 2-cells obtained from elementary 2-cells and their inverses by applying morphisms in the theory of theories.

Proof: From the previous Lemma we have 2-cells

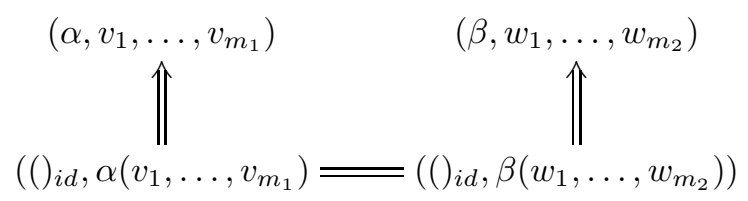

of the prescribed type. One obtains the desired result by inverting the 2-cell on the left. 
Theorem 6.5. There is a bijection between the set of small pseudo T-algebras and the set of small $\mathcal{T}$-algebras.

Proof: $\quad$ Let $(X, \Phi)$ be a small pseudo $T$-algebra. Define a morphism $\Psi: \mathcal{T} \rightarrow$ $\operatorname{End}(X)$ of theories enriched over groupoids by the following sequence of functors $\Psi_{n}: \mathcal{T}(n) \rightarrow \operatorname{End}(X)(n)$. For notational convenience, the subscript $n$ is usually left off below. For $\left(\alpha, w_{1}, \ldots, w_{\ell}\right) \in \mathcal{T}(n)$ define

$$
\Psi\left(\alpha, w_{1}, \ldots, w_{\ell}\right):=\alpha\left(\Phi\left(w_{1}\right), \ldots, \Phi\left(w_{\ell}\right)\right) .
$$

For elementary 2-cells, define

$$
\begin{aligned}
\Psi\left(c_{w, w_{1}, \ldots, w_{k}}\right) & :=c_{w, w_{1}, \ldots, w_{k}} \\
\Psi(I) & :=I \\
\Psi\left(s_{w, f}\right) & :=s_{w, f}
\end{aligned}
$$

where the symbols on the right denote the coherence natural isomorphisms from the pseudo $T$-algebra structure.

If $\alpha$ is a word in the theory of theories and $\varepsilon_{1}, \ldots, \varepsilon_{k}$ are elementary 2-cells, then

$$
\Psi\left(\alpha\left(\varepsilon_{1}, \ldots, \varepsilon_{k}\right)\right):=\alpha\left(\Psi\left(\varepsilon_{1}\right), \ldots, \Psi\left(\varepsilon_{k}\right)\right) .
$$

This is well defined, because if $\alpha\left(\varepsilon_{1}, \ldots, \varepsilon_{k}\right)=\beta\left(\varepsilon_{1}, \ldots, \varepsilon_{k}\right)$ with $\varepsilon_{1}, \ldots, \varepsilon_{k}$ elementary, then $\alpha=\beta$.

Consider the 2-cell

$$
\left(()_{i d_{n}}, \alpha\left(v_{1}, \ldots, v_{m}\right)\right) \Longrightarrow\left(\alpha, v_{1}, \ldots, v_{m}\right)
$$

for some $\alpha \in \mathbf{R}_{n}(\bar{m})$. By the above lemma, the word $\alpha$ can be expressed in the form $\sigma_{s} \odot \cdots \odot \sigma_{1}$ where each $\sigma_{r}$ is obtained from a tuple of elementary 2-cells by applying a morphism in $\mathbf{R}$. Define

$$
\Psi\left(\sigma_{s} \odot \cdots \odot \sigma_{1}\right):=\Psi\left(\sigma_{s}\right) \odot \cdots \odot \Psi\left(\sigma_{1}\right)
$$

where each $\Psi\left(\sigma_{r}\right)$ is defined as in the previous paragraph. To see that this is well defined, suppose $\sigma_{s} \odot \cdots \odot \sigma_{1}=\sigma_{s^{\prime}}^{\prime} \odot \cdots \odot \sigma_{1}^{\prime}$ where each $\sigma_{r^{\prime}}^{\prime}$ is obtained from a tuple of elementary 2-cells by applying a morphism in $\mathbf{R}$. Such a sequence gives rise to an expression $\alpha=\alpha_{s^{\prime}}^{\prime} \circ \cdots \circ \alpha_{1}^{\prime}$ where $\alpha_{1}^{\prime}, \ldots, \alpha_{s^{\prime}}^{\prime}$ are tuples of generating morphisms. Let $\alpha=\alpha_{s} \circ \cdots \circ \alpha_{1}$ be the expression that gave rise to $\sigma_{s} \odot \cdots \odot \sigma_{1}$. It suffices to consider the case

$$
\alpha=\alpha_{4} \circ \alpha_{3} \circ \alpha_{2} \circ \alpha_{1}=\alpha_{4} \circ \alpha_{3}^{\prime} \circ \alpha_{2}^{\prime} \circ \alpha_{1}
$$

with $\alpha_{3} \circ \alpha_{2}=\alpha_{3}^{\prime} \circ \alpha_{2}^{\prime}$ because $\alpha_{s^{\prime}}^{\prime} \circ \cdots \circ \alpha_{1}^{\prime}$ can be obtained from $\alpha_{s} \circ \cdots \circ \alpha_{1}$ by a finite number of applications of the relations in the theory of theories. Then we have the following diagram, whose vertical columns are $\Psi\left(\sigma_{4} \odot \sigma_{3} \odot \sigma_{2} \odot \sigma_{1}\right)$ and 
$\Psi\left(\sigma_{4} \odot \sigma_{3}^{\prime} \odot \sigma_{2}^{\prime} \odot \sigma_{1}\right)$ respectively.

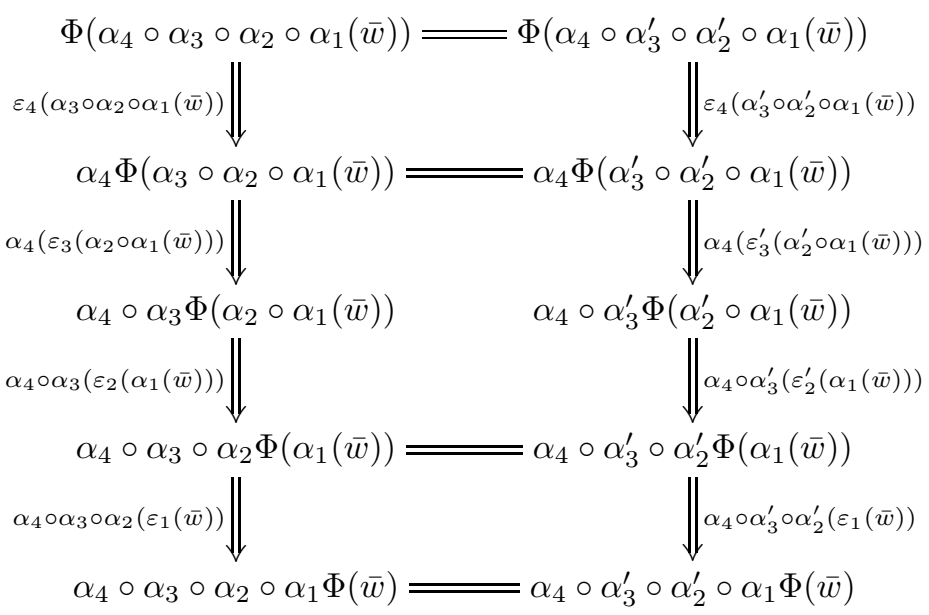

Here $\varepsilon_{i}$ denotes the tuple of elementary 2-cells needed to bring $\alpha_{i}$ past $\Phi$. The inner square commutes because of the coherence diagrams. The top and bottom squares commute because $\alpha_{3} \circ \alpha_{2}=\alpha_{3}^{\prime} \circ \alpha_{2}^{\prime}$. Hence

$$
\Psi\left(\sigma_{4} \odot \sigma_{3} \odot \sigma_{2} \odot \sigma_{1}\right)=\Psi\left(\sigma_{4} \odot \sigma_{3}^{\prime} \odot \sigma_{2}^{\prime} \odot \sigma_{1}\right)
$$

and $\Psi$ is well defined on any 2-cell of the form

$$
\left(()_{i d_{n}}, \alpha\left(v_{1}, \ldots, v_{m}\right)\right) \Longrightarrow\left(\alpha, v_{1}, \ldots, v_{m}\right) .
$$

Next one must define $\Psi$ on 2-cells of the form

$$
\left(\alpha, v_{1}, \ldots, v_{m_{1}}\right) \Longrightarrow\left(\beta, w_{1}, \ldots, w_{m_{2}}\right) .
$$

According to Lemma 6.3 we have 2-cells

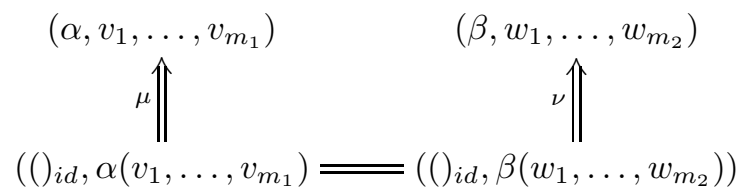

on which $\Psi$ is already defined. Define

$$
\Psi\left(\nu \odot \mu^{-1}\right):=\Psi(\nu) \odot \Psi(\mu)^{-1} .
$$

To see that this is well defined, suppose

$$
\sigma_{s} \odot \cdots \odot \sigma_{1}:\left(\alpha, v_{1}, \ldots, v_{m_{1}}\right) \Longrightarrow\left(\beta, w_{1}, \ldots, w_{m_{2}}\right)
$$

is another expression where each $\sigma_{r}$ is obtained by applying a morphism of $\mathbf{R}$ to a tuple of elementary 2-cells or their inverses. Then

$$
\begin{aligned}
\Psi(\nu) & =\Psi\left(\sigma_{s} \odot \cdots \odot \sigma_{1} \odot \mu\right) \\
\Psi(\nu) & =\Psi\left(\sigma_{s} \odot \cdots \odot \sigma_{1}\right) \odot \Psi(\mu) \\
\Psi(\nu) \odot \Psi\left(\mu^{-1}\right) & =\Psi\left(\sigma_{s} \odot \cdots \odot \sigma_{1}\right) \\
\Psi\left(\nu \odot \mu^{-1}\right) & =\Psi\left(\sigma_{s} \odot \cdots \odot \sigma_{1}\right)
\end{aligned}
$$

and $\Psi$ is well defined on 2-cells. 
By construction $\Psi_{n}: \mathcal{T}(n) \rightarrow \operatorname{End}(X)(n)$ is a functor and it preserves $\gamma,()_{g}$, and $(1, *)=1$. Hence $X$ is a $\mathcal{T}$-algebra with structure map given by $\Psi$. This procedure $\Phi \mapsto \Psi$ defines a map

$$
\text { Pseudo } T \text {-Algebras } \rightarrow \mathcal{T} \text {-Algebras. }
$$

Now I define a map

$$
\mathcal{T} \text {-Algebras } \rightarrow \text { Pseudo } T \text {-Algebras. }
$$

Let $(X, \Psi)$ be a $\mathcal{T}$-algebra. Then define natural isomorphisms

$$
\begin{aligned}
c_{w, w_{1}, \ldots, w_{k}} & :=\Psi\left(c_{w, w_{1}, \ldots, w_{k}}\right) \\
I & :=\Psi(I) \\
s_{w, f} & :=\Psi\left(s_{w, f}\right)
\end{aligned}
$$

where the symbols $c, I, s$ on the right are 2 -cells in $\mathcal{T}$. Also define

$$
\Phi_{n}(w):=\Psi_{n}\left(()_{i d_{n}}, w\right)
$$

for $w \in T(n)$. Then the coherence diagrams are satisfied because $\Psi_{n}: \mathcal{T}(n) \rightarrow$ $\operatorname{End}(X)(n)$ is a functor for every $n$ and $\Psi$ preserves $\gamma,()_{g}$, and 1 .

One can easily check that the two procedures are inverse to one another and that they define a bijection.

Next we can define a 2-monad $C: C a t \rightarrow C a t$ like on page 43 Define a 2-functor $C$ by

$$
C X:=\frac{\left(\bigcup_{n \geq 0}\left(\mathcal{T}(n) \times X^{n}\right)\right)}{\Gamma}
$$

for any small category $X$. One can similarly define 2-natural transformations $\eta$ : $1_{C a t} \Rightarrow C$ and $\mu: C^{2} \Rightarrow C$.

Theorem 6.6. Let $\mathcal{C}_{C}$ denote the 2-category of small strict $C$-algebras, pseudo morphisms, and 2-cells. Let $\mathcal{C}_{T}$ denote the 2-category of small pseudo T-algebras. Then $\mathcal{C}_{C}$ and $\mathcal{C}_{T}$ are 2-equivalent.

Proof: $\quad$ The small $C$-algebras are precisely the small $\mathcal{T}$-algebras by a proof similar to Theorem 5.11 But by the previous theorem, the small $\mathcal{T}$-algebras are precisely the pseudo $T$-algebras. To see that the morphisms of the 2 -categories $\mathcal{C}_{C}$ and $\mathcal{C}_{T}$ are the same, one must compare the coherence isos of the morphisms. They are related by

$$
\rho_{\left(\alpha, w_{1}, \ldots, w_{k}\right) \times(\bar{x})}^{C}=\alpha\left(\rho_{w_{1}}^{T}, \ldots, \rho_{w_{k}}^{T}\right)(\bar{x}) .
$$

In diagram (1) of Definition 6.2 the right vertical composition can be replaced by the appropriate component of $\rho^{C}$ by the composition coherence diagram for coherence isos of pseudo morphisms of $C$-algebras. Then (1) commutes by naturality of $\rho^{C}$. In (2) of Definition 6.2 the right vertical equality can be replaced by the appropriate component of $\rho^{C}$ by the unit coherence diagram for coherence isos of pseudo morphisms of $C$-algebras. Then (2) commutes by the naturality of $\rho^{C}$. Diagram (3) commutes by the naturality of $\rho^{C}$. The 2-cells of the 2 -categories $\mathcal{C}_{C}$ and $\mathcal{C}_{T}$ are also the same.

Power's Theorem 5.3 in 43 states that the 2-category of strict $C$-algebras, pseudo morphisms and 2-cells is bi-equivalent to the 2-category of strict $\mathcal{T}$-algebras, pseudo morphisms, and 2-cells where $\mathcal{T}$ is a theory enriched in categories and $C$ is the corresponding 2-monad in his construction. Power's theorem differs from the 
above Theorem [6.6] in several regards. Theorem [6.6] above uses strict $C$-algebras to describe pseudo $T$-algebras, where $T$ is a usual theory. Theorem 6.6 also has a 2-equivalence rather than a biequivalence.

Finally, the 2-equivalence of Theorem 5.20 yields the desired 2-equivalence.

A reformulation of Theorem 2.6 of 9 in our language is the following.

Theorem 6.7. Let $C$ be a 2-monad. Then the 2-category of small strict $C$-algebras, pseudo morphisms, and 2-cells of pseudo morphisms admits strictly weighted pseudo limits of strict 2-functors.

We conclude the following completeness theorem from the result of Blackwell, Kelly, and Power.

Theorem 6.8. Let $T$ be a theory. Then the 2-category of pseudo T-algebras admits strictly weighted pseudo limits of strict 2-functors.

Proof: A 2-equivalence of 2-categories preserves weighted pseudo limits because it admits a left 2-adjoint. Then the result follows from the previous two theorems.

\section{Weighted Pseudo Limits in the 2-Category of Pseudo T-Algebras}

In this section we prove that for any fixed theory $T$ the 2-category of pseudo $T$ algebras introduced in the previous section has pseudo limits. The proof in section [4 of pseudo limits in the 2-category of small categories is modified below to fit the pseudo algebra case. Let $\mathcal{C}$ denote the 2-category of pseudo $T$-algebras in this section. The existence of cotensor products in $\mathcal{C}$ allows us to conclude that $\mathcal{C}$ admits all weighted pseudo limits from a theorem of Street. This result is more general than Theorem 6.8 because it allows the functors to be lax. The proof in this section for pseudo limits is also constructive, whereas Theorem 6.8 is not.

Theorem 7.1. The 2-category $\mathcal{C}$ of small pseudo T-algebras has pseudo limits.

Proof: $\quad$ Let $\mathcal{J}$ be a small 1-category and $F: \mathcal{J} \rightarrow \mathcal{C}$ a pseudo functor. Let 1 denote the terminal object of the 2-category of small categories as before. Let $U$ denote the forgetful 2 -functor from the 2-category $\mathcal{C}$ of pseudo $T$-algebras to the 2-category of small categories. The candidate for the pseudo limit of $F$ is $L:=$ PseudoCone $(\mathbf{1}, U \circ F)$ as before. Note that these are pseudo cones into the 2 -category of small categories, not into the 2 -category of pseudo $T$-algebras. Define $\pi: \Delta_{L} \Rightarrow F$ as before. I must show that $L$ has the structure of a pseudo $T$-algebra, that $\pi$ is a pseudo natural transformation to $F$, and that $L$ and $\pi$ are universal. These proofs will draw on the analogous results for the pseudo limit of $U \circ F$.

Lemma 7.2. The small category $L$ has the structure of a pseudo T-algebra.

Proof: We first make the identification of the categories $P$ and $L$ as in Remarks 4.4 and 4.5] Let $\eta^{\ell}=\left(a_{i}^{\ell}\right)_{i} \times\left(\varepsilon_{f}^{\ell}\right)_{f} \in \operatorname{ObjL}$ and $\left(\xi_{i}^{\ell}\right)_{i} \in M o r L$ for $1 \leq \ell \leq n$ and $w \in T(n)$. Denote the action of the theory $T$ on $F i=A_{i}$ by $\Phi_{i}$ for all $i \in \operatorname{Obj} \mathcal{J}$. Define $a_{i}:=\Phi_{i}(w)\left(a_{i}^{1}, \ldots, a_{i}^{n}\right)$ and $\varepsilon_{f}:=\Phi_{T f}(w)\left(\varepsilon_{f}^{1}, \ldots, \varepsilon_{f}^{n}\right) \circ \rho_{w}^{F f}\left(a_{S f}^{1}, \ldots, a_{S f}^{n}\right)$ : $F f\left(a_{S f}\right) \rightarrow a_{T f}$ as well as $\xi_{i}:=\Phi_{i}(w)\left(\xi_{i}^{1}, \ldots, \xi_{i}^{n}\right)$. Then the action $\Phi$ of $T$ on $L$ is defined by $\Phi(w)\left(\eta^{1}, \ldots, \eta^{n}\right):=\left(a_{i}\right)_{i} \times\left(\varepsilon_{f}\right)_{f}$ and $\Phi(w)\left(\left(\xi_{i}^{1}\right)_{i}, \ldots,\left(\xi_{i}^{n}\right)_{i}\right):=\left(\xi_{i}\right)_{i}$. It must be verified that these outputs belong to $L$. 
I claim that $\left(a_{i}\right)_{i} \times\left(\varepsilon_{f}\right)_{f} \in O b j L$. I prove this by verifying the coherences in Remarks 4.4 and 4.5 for a fixed word $w \in T(2)$. To avoid cumbersome notation, I write + for the action of the word $w$ in any pseudo $T$-algebra. The verification for a general word is the same. I abbreviate $\rho_{w}^{H}$ as $\rho^{H}$ for any morphism $H$ of pseudo $T$-algebras. The only word appearing in the following diagrams is $w$, so there is no ambiguity. Let $\gamma_{f, g}:=\gamma_{f, g}^{F}$ and $\delta_{j}:=\delta_{j}^{F}$. First I show that for all $j \in O b j \mathcal{J}$ the diagram.

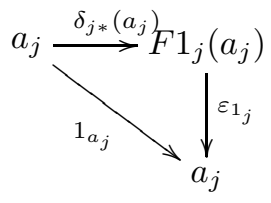

commutes where $a_{j}=a_{j}^{1}+a_{j}^{2}$ and $\varepsilon_{1_{j}}=\left(\varepsilon_{1_{j}}^{1}+\varepsilon_{1_{j}}^{2}\right) \circ \rho^{F 1_{j}}\left(a_{j}^{1}, a_{j}^{2}\right)$ as defined above. After writing this diagram out we get

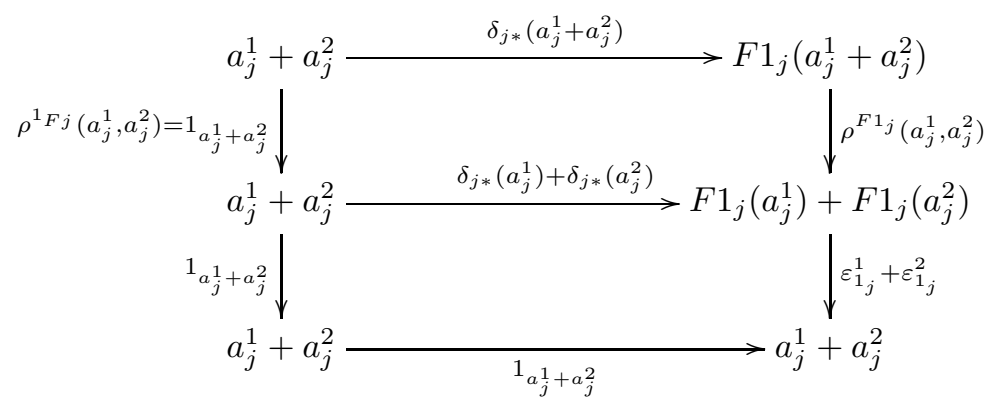

where the top horizontal arrow is $\delta_{j *}\left(a_{j}\right)$ and the right vertical composition is $\varepsilon_{1_{j}}$ by definition. The top square commutes because $\delta_{j *}: 1_{F j} \Rightarrow F 1_{j}$ is a 2-cell in the 2-category $\mathcal{C}$. The bottom square commutes because + is a functor and $\varepsilon_{1_{j}}^{\ell} \circ \delta_{j *}\left(a_{j}^{\ell}\right)=1_{a_{j}^{\ell}}$ for $\ell=1,2$. Hence (7) commmutes. Next I show that for all $i \stackrel{f}{\longrightarrow} j \stackrel{g}{\longrightarrow} k$ in $\mathcal{J}$ the diagram

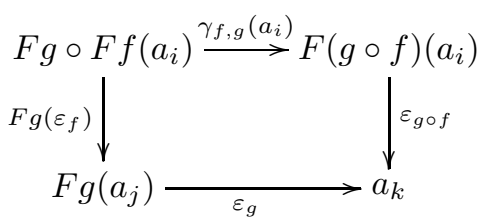


commutes where $\varepsilon_{f}=\varepsilon_{f}^{1}+\varepsilon_{f}^{2}$ etc. After writing out this diagram we get

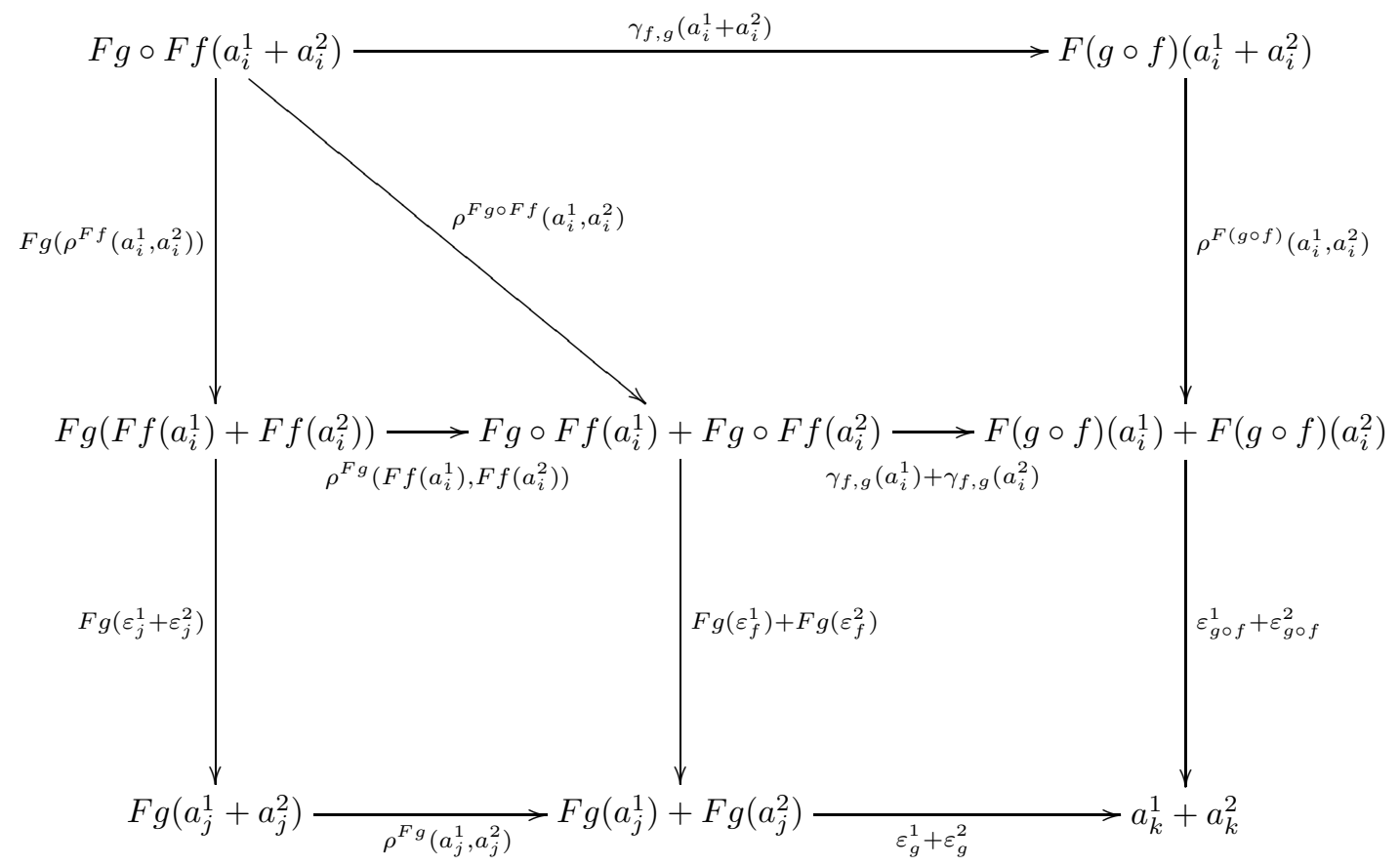

where the outermost square is (8). The upper left triangle commutes by the definition of composition of pseudo $T$-algebras. The upper right quadrilateral commutes because $\gamma_{f, g}: F g \circ F f \Rightarrow F(g \circ f)$ is a 2-cell in the 2-category of pseudo $T$-algebras. The lower left square commutes because $\rho^{F g}: F g(+) \Rightarrow F g+F g$ is a natural transformation. The bottom right square commutes because + is a functor and $\varepsilon_{g}^{\ell} \circ\left(F g\left(\varepsilon_{f}^{\ell}\right)=\varepsilon_{g \circ f}^{\ell} \circ \gamma_{f, g}\left(a_{i}^{\ell}\right)\right.$ for $\ell=1,2$. Thus all four inner diagrams commute and (8) commutes. Thus both coherences in Remark 4.4 are satisfied and $\eta^{1}+\eta^{2}=\left(a_{i}\right)_{i} \times\left(\varepsilon_{f}\right)_{f}$ is an object of $L$.

I claim that $\left(\xi_{i}^{1}\right)_{i}+\left(\xi_{i}^{2}\right)=\left(\xi_{i}\right)_{i}$ is a morphism of $L$ where $\left(\xi_{i}^{1}\right)_{i}:\left(a_{i}^{1}\right)_{i} \times\left(\varepsilon_{f}^{1}\right)_{f} \rightarrow$ $\left(b_{i}^{1}\right)_{i} \times\left(\zeta_{f}^{1}\right)_{f}$ and $\left(\xi_{i}^{2}\right)_{i}:\left(a_{i}^{2}\right)_{i} \times\left(\varepsilon_{f}^{2}\right)_{f} \rightarrow\left(b_{i}^{2}\right)_{i} \times\left(\zeta_{f}^{2}\right)_{f}$ are morphisms in $L$. In other words I must show that

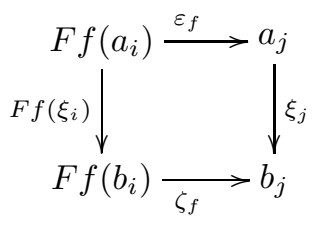


commutes for all morphisms $f: i \rightarrow j$ in $\mathcal{J}$, where $a_{i}=a_{i}^{1}+a_{i}^{2}$ etc. If we write out the diagram we get

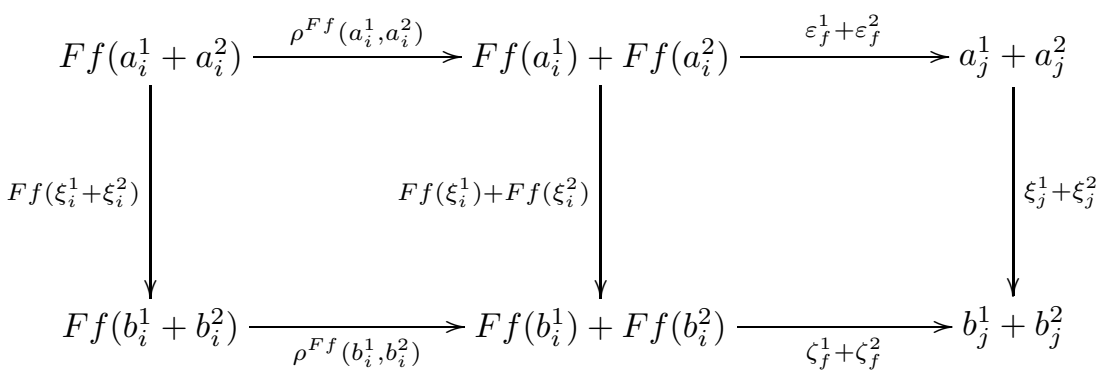

where the outermost square is (9). The square on the left commutes because $\rho^{F f}$ : $F f(+) \Rightarrow F f+F f$ is a natural transformation. The right square commutes because the diagram

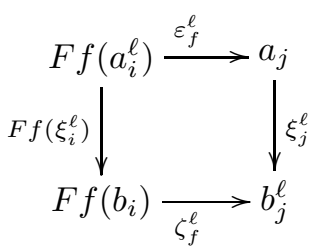

commutes for $\ell=1,2$ and because + is a functor. Hence $\left(\xi_{i}^{1}\right)_{i}+\left(\xi_{i}^{2}\right)=\left(\xi_{i}\right)_{i}$ is a morphism of $L$. Thus $\Phi(w): L \times L \rightarrow L$.

The map $\Phi(w)$ preserves compositions and identities because the individual components do. Thus $\Phi(w): L \times L \rightarrow L$ is a functor. The same argument works for words in $T(n)$ for all $n \in \mathbb{N}$. Thus $\Phi$ defines an action of the theory $T$ on the small category $L$.

We define the coherence isos for $\Phi$ to be those maps which have the coherence isos of $\Phi_{i}$ in the $i$-th component. One can prove that they are morphisms of the category $L$, i.e. satisfy the diagram in Remark 4.5 by using the coherence diagrams of $\rho$ with the respective coherence iso as well as the naturality of the individual components. The coherence isos for $\Phi$ are natural because they are natural in each component. The coherence isos for $\Phi$ satisfy the coherence diagrams because the individual components do. Thus $L$ is a pseudo $T$-algebra with the action of the theory $T$ given by $\Phi$.

Lemma 7.3. The map $\pi: \Delta_{L} \Rightarrow F$ is a pseudo natural transformation with the coherence 2 -cells determined by $\tau$.

Proof: It is clear from the work on the small category case that $\pi$ is a pseudo natural transformation when we forget all the pseudo $T$-algebra structures. Therefore it suffices to show that $\pi_{j}: L \rightarrow F j$ is a morphism of pseudo $T$-algebras for all $j \in O b j \mathcal{J}$ and that $\tau_{i, j}(f): F f \circ \pi_{i} \Rightarrow \pi_{j}$ is a 2-cell in the 2-category of pseudo $T$-algebras for all morphisms $f: i \rightarrow j$ in $\mathcal{J}$.

Let $j \in \operatorname{Obj} \mathcal{J}$. Then $\pi_{j}: L \rightarrow F j$ is a functor. We abbreviate the action of $w \in T(2)$ by + as above. Then for $\eta^{\ell}=\left(a_{i}^{\ell}\right)_{i} \times\left(\varepsilon_{f}^{\ell}\right)_{f} \in \operatorname{ObjL}$ for $\ell=1,2$ we have $\pi_{j}\left(\eta^{1}+\eta^{2}\right)=\pi_{j}\left(\left(a_{i}^{1}+a_{i}^{2}\right)_{i} \times\left(\left(\varepsilon_{f}^{1}+\varepsilon_{f}^{2}\right) \circ \rho_{w}^{F f}\left(a_{S f}^{1}, a_{S f}^{2}\right)\right)_{f}\right)=a_{j}^{1}+a_{j}^{2}=\pi_{j}\left(\eta^{1}\right)+\pi_{j}\left(\eta^{2}\right)$. The same calculation works for words in $T(n)$ for all $n \in \mathbb{N}$. We conclude that $\pi_{j}$ 
commutes with the action of $T$. If we take $\rho_{w}^{\pi_{j}}=i_{\pi_{j}} \circ i_{\Phi(w)}$ then $\pi_{j}$ is a morphism of pseudo $T$-algebras for all $j \in \mathcal{J}$.

Let $f: i \rightarrow j$ be a morphism of $L$. To show that $\tau_{i, j}(f)$ is a 2 -cell, we must show that the diagram

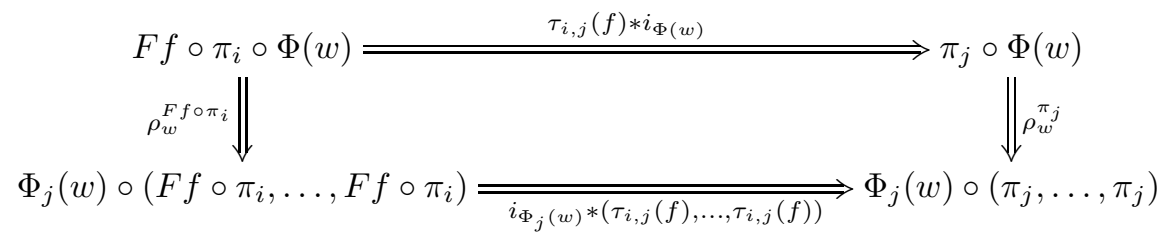

commutes for all words $w$. Recalling that $\tau_{i, j}(f)_{\eta}:=\tau_{i, j}^{\eta}(f)$ and evaluating the diagram on $\left(\eta^{1}, \eta^{2}\right)$ where $\eta^{\ell}=\left(a_{i}^{\ell}\right)_{i} \times\left(\varepsilon_{f}^{\ell}\right)_{f} \in \operatorname{Obj} L$ for $\ell=1,2$ gives

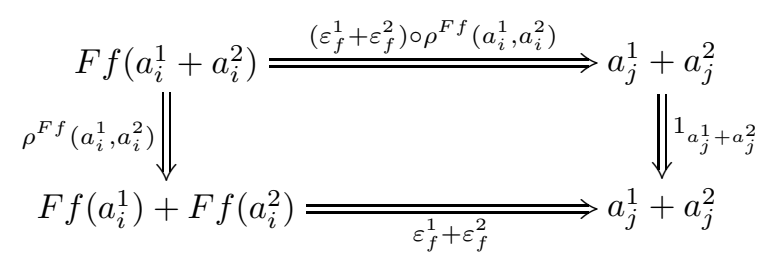

which obviously commutes. Hence $\tau_{i, j}(f)$ is a 2-cell in the 2-category of pseudo $T$-algebras for all $f: i \rightarrow j$ and $\pi$ is a pseudo natural transformation.

Now I must show that the pseudo $T$-algebra $L$ and the pseudo natural transformation $\pi: \Delta_{L} \Rightarrow F$ are universal in the sense that the functor $\phi: \operatorname{Mor}_{\mathcal{C}}(V, L) \rightarrow$ PseudoCone $(V, F)$ as defined in the small category case is an isomorphism of categories for all objects $V$ of $\mathcal{C}$. In the following, $V$ is a fixed object of the 2-category $\mathcal{C}$ of pseudo $T$-algebras.

Lemma 7.4. The map $\phi: \operatorname{Mor}_{\mathcal{C}}(V, L) \rightarrow$ PseudoCone $(V, F)$ is a functor.

Proof: The proof is analogous to the proof for the $\phi$ of the pseudo colimit of small categories in Lemma 3.3. The only difference is that here we have to verify that $\tau_{i, j}(f) * i_{b}$ is a 2 -cell of the 2-category $\mathcal{C}$ of pseudo $T$-algebras for any morphism $b: V \rightarrow L$ as in the comments just before Lemma 3.3. But that is immediate because $i_{b}$ is obviously a 2-cell and the horizontal composition of 2-cells is again a 2 -cell.

Now I construct a functor $\psi: \operatorname{PseudoCone}(V, F) \Rightarrow \operatorname{Mor}_{\mathcal{C}}(V, L)$ that is inverse to $\phi$. First I define $\psi$ for objects, then for morphisms. Finally I verify that it is a functor and inverse to $\phi$. The next two lemmas define a morphism $\psi\left(\pi^{\prime}\right): V \rightarrow L$ in $\mathcal{C}$ for any object $\pi^{\prime}$ of PseudoCone $(V, F)$.

Lemma 7.5. Let $\pi^{\prime}: \Delta_{V} \Rightarrow F$ be a pseudo natural transformation with coherence 2-cells $\tau^{\prime}$. For any fixed $x \in \operatorname{ObjV}$ we have $\psi\left(\pi^{\prime}\right)(x):=b(x):=\left(\pi_{i}^{\prime}(x)\right)_{i} \times$ $\left(\tau_{S f, T f}^{\prime}(f)_{x}\right)_{f}$ is an element of $\operatorname{ObjL}$.

Proof: This follows from Lemma 4.7 by forgetting the pseudo $T$-algebra structures. Thus $\psi\left(\pi^{\prime}\right)(x) \in \operatorname{ObjL}$. 
Lemma 7.6. Let $\pi^{\prime}: \Delta_{V} \Rightarrow F$ be a pseudo natural transformation with coherence 2-cells $\tau^{\prime}$. Then for any fixed $h \in \operatorname{Mor}_{V}(x, y)$ we have a modification $\psi\left(\pi^{\prime}\right)(h):=$ $b(h):=\left(\pi_{i}^{\prime}(h)\right)_{i}: b(x) \rightsquigarrow b(y)$. This notation means $b(h)_{i}(*):=\pi_{i}^{\prime}(h)$.

Proof: This is exactly the same as the proof of Lemma 4.8 because the pseudo $T$-algebra structure on $L$ makes no additional requirements on the morphisms of the small category $L$.

Lemma 7.7. For any pseudo natural transformation $\pi^{\prime}: \Delta_{V} \Rightarrow F$ the map $\psi\left(\pi^{\prime}\right)=$ $b: V \rightarrow L$ as defined above is a morphism of pseudo T-algebras.

Proof: By Lemma 4.9 the map $b: V \rightarrow L$ is a functor between the underlying small categories. I define a natural transformation $\rho_{w}^{b}$ for $w \in T(2)$ and abbreviate the action by + . Define $\rho_{w}^{b}\left(x_{1}, x_{2}\right):=\rho^{b}\left(x_{1}, x_{2}\right):=\left(\rho^{\pi_{i}^{\prime}}\left(x_{1}, x_{2}\right)\right)_{i}: b\left(x_{1}+x_{2}\right) \rightarrow$ $b\left(x_{1}\right)+b\left(x_{2}\right)$ for all $x_{1}, x_{2} \in \operatorname{ObjV}$. I claim that $\rho^{b}\left(x_{1}, x_{2}\right)$ is a morphism of $L$. Let $\tau_{i, j}^{\prime}(f)$ denote the 2-cell associated to $\pi^{\prime}: \Delta_{V} \Rightarrow F$ for $f: i \rightarrow j$ in $\mathcal{J}$. Since $\tau_{i, j}^{\prime}(f): F f \circ \pi^{\prime} \Rightarrow \pi_{j}^{\prime}$ is a 2-cell, we know that

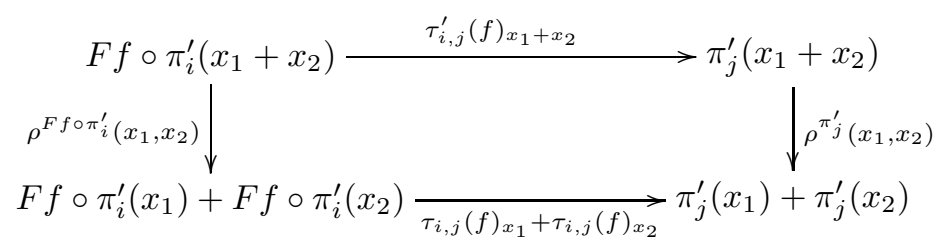

commutes. Rewriting the left vertical arrow and the bottom arrow gives

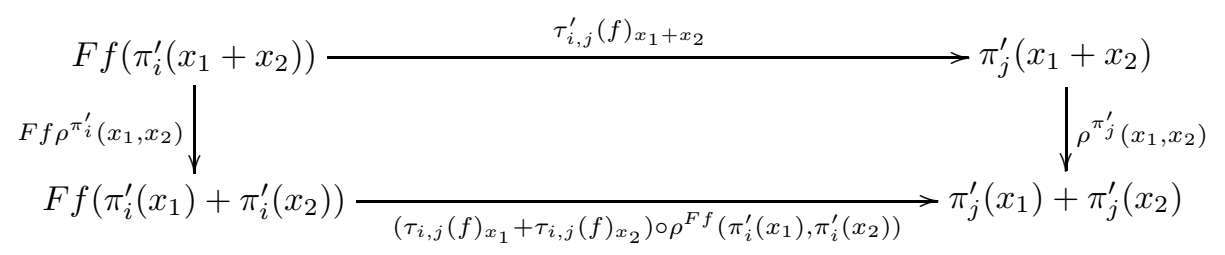

which states precisely that $\rho^{b}\left(x_{1}, x_{2}\right)=\left(\rho^{\pi_{i}^{\prime}}\left(x_{1}, x_{2}\right)\right)_{i}: b\left(x_{1}+x_{2}\right) \rightarrow b\left(x_{1}\right)+b\left(x_{2}\right)$ is a morphism in $L$ by Remark 4.5 The map $\rho^{b}$ is natural because each component is natural. Hence $\rho^{b}$ is a natural transformation. If we define $\rho_{w}^{b}$ analogously for arbitrary words $w$ of the theory $T$, then the coherences of Definition 6.1 are satisfied because they are satisfied componentwise. Hence $\psi\left(\pi^{\prime}\right)=b: V \rightarrow L$ is a morphism of pseudo $T$-algebras.

Lemma 7.8. Let $\Xi: \alpha \rightsquigarrow \beta$ be a morphism of the category PseudoCone $(V, F)$. Then $\psi(\Xi): \psi(\alpha) \Rightarrow \psi(\beta)$ defined by $V \ni x \mapsto\left(\Xi_{i}(x)\right)_{i} \in \operatorname{Mor}_{L}(\psi(\alpha) x, \psi(\beta) x)$ is a 2-cell in the 2-category of pseudo T-algebras. As in Lemma 4.10 this definition means $\psi(\Xi)(x)_{i}(*):=\Xi_{i}(x)$. 
Proof: The map $\psi(\Xi)$ is a natural transformation by Lemma 4.10 For all $i \in \operatorname{Obj} \mathcal{J}$ we have morphisms $\alpha_{i}, \beta_{i}: V \rightarrow F i$ and 2-cells $\Xi_{i}: \alpha_{i} \Rightarrow \beta_{i}$. Hence

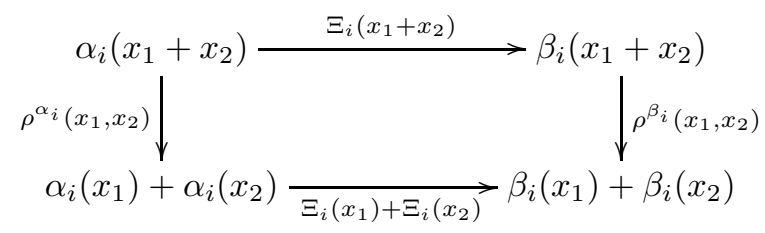

commutes. Since these are the components for $\psi(\alpha)(x), \psi(\beta)(x)$, and $\psi(\Xi)(x)$, we see that

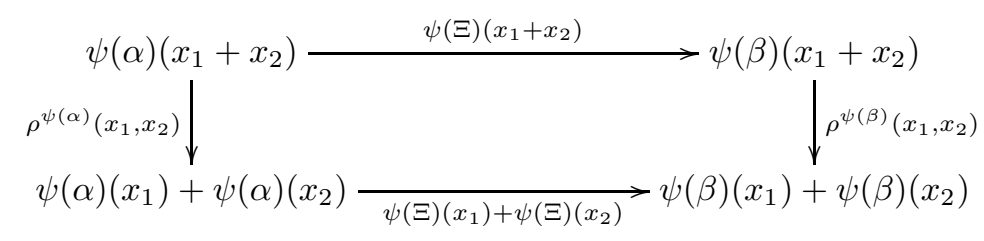

commutes. Similar diagrams hold for arbitrary words $w$ in the theory $T$. Thus $\psi(\Xi)$ is a 2 -cell.

Theorem 7.9. The map $\psi:$ PseudoCone $(V, F) \rightarrow \operatorname{Mor}_{\mathcal{C}}(V, L)$ as defined in the previous lemmas is an inverse functor to $\phi$.

Proof: This follows from the calculations of Theorem 4.11 and Lemmas 4.12 and 4.13

Lemma 7.10. The pseudo T-algebra $L$ with the pseudo cone $\pi: \Delta_{L} \Rightarrow F$ is the pseudo limit of the pseudo functor $F: \mathcal{J} \rightarrow \mathcal{C}$.

Proof: The functor $\phi: \operatorname{Mor}_{\mathcal{C}}(V, L) \rightarrow \operatorname{PseudoCone}(V, F)$ is an isomorphism of categories by the previous lemmas. Since $V$ was an arbitrary object of $\mathcal{C}$ we conclude that $L$ and $\pi$ are universal.

Thus every pseudo functor $F: \mathcal{J} \rightarrow \mathcal{C}$ from a small 1-category $\mathcal{J}$ to the 2 category $\mathcal{C}$ of pseudo $T$-algebras has a pseudo limit. Hence $\mathcal{C}$ has pseudo limits. This completes the proof of Theorem 7.1 .

Theorem 7.11. The 2-category of pseudo T-algebras admits bilimits.

Proof: It admits pseudo limits and therefore admits bilimits.

Lemma 7.12. The 2-category $\mathcal{C}$ of pseudo T-algebras admits cotensor products.

Proof: $\quad$ Let $J \in O b j C a t$ and let $F$ be a pseudo $T$-algebra. Let $U: \mathcal{C} \rightarrow C a t$ be the forgetful functor. Define $P:=(U F)^{J}$, which is the 1-category of 1-functors $J \rightarrow U F$. I claim that $P$ has the structure of a pseudo $T$-algebra. Let $\Phi_{n}: T(n) \rightarrow$ Functors $\left(F^{n}, F\right)$ denote the structure maps for $F$. Define $\Phi_{n}^{P}: T(n) \rightarrow$ Functors $\left(P^{n}, P\right)$ by

$$
\Phi_{n}^{P}(w)\left(p_{1}, \ldots, p_{n}\right)(j):=\Phi_{n}(w)\left(p_{1}(j), \ldots, p_{n}(j)\right)
$$


for $j \in O b j J$ and $p_{1}, \ldots, p_{n} \in O b j P$. Coherence isos are defined analogously. For example, define

$s_{w, f}^{P}: \Phi^{P}\left(w_{f}\right) \Rightarrow \Phi^{P}(w)_{f}$ for $f: m \rightarrow n$ on $p_{1}, \ldots, p_{n} \in O b j P$ as the 1-natural transformation

$$
s_{w, f}^{P}\left(p_{1}, \ldots, p_{n}\right): \Phi_{n}^{P}\left(w_{f}\right)\left(p_{1}, \ldots, p_{n}\right) \Longrightarrow \Phi_{n}^{P}(w)_{f}\left(p_{1}, \ldots, p_{n}\right)
$$

which is $s_{w, f}^{P}\left(p_{1}, \ldots, p_{n}\right)(j):=s_{w, f}\left(p_{1}(j), \ldots, p_{n}(j)\right)$ for $j \in O b j J$. Then all coherence diagrams are satisfied because they are satisfied pointwise. Hence, $P$ has the structure of a pseudo $T$-algebra.

I claim that $P$ is a cotensor product of $J$ and $F$. We use Remark 2.1] Define a functor $\pi: J \rightarrow \mathcal{C}(P, F)$ by

$$
\begin{aligned}
& \pi(j)(p):=p(j) \\
& \pi(j)(\eta):=\eta(j) \\
& \pi(g)(p):=p(g)
\end{aligned}
$$

for $j$ an object of $J, p$ a a functor from $J$ to $U F, \eta$ a natural transformation, and $g$ a morphism of $J$. Let $\sigma: J \rightarrow \mathcal{C}(C, F)$ be a functor. Define a morphism $b: C \rightarrow P$ of pseudo $T$-algebras by

$$
\begin{aligned}
b(c)(j) & :=\sigma(j)(c) \\
b(c)(f) & :=\sigma(f)(c) \\
b(m)(j) & :=\sigma(j)(m)
\end{aligned}
$$

for $c \in \operatorname{ObjC}, j \in \operatorname{Obj} J, f \in M o r J$, and $m \in \operatorname{Mor} C$. Then $b$ is strict and it is the unique morphism $C \rightarrow P$ such that $\mathcal{C}(b, F) \circ \pi=\sigma$. A similar argument can be made for 2-cells. Thus $P$ is a cotensor product of $J$ and $F$ with unit $\pi$.

Theorem 7.13. The 2-category $\mathcal{C}$ of pseudo $T$-algebras admits all weighted pseudo limits.

Proof: By Theorem 7.1 it admits pseudo limits, and hence it admits pseudo equalizers. The 2 -category $\mathcal{C}$ obviously admits products. By Lemma 7.12 it admits cotensor products. Hence by Theorem [2.4] it admits all weighted pseudo limits.

\section{Bi-universal Arrows and Bi-ADjoints}

After studying bilimits and bicolimits, we turn our attention to another type of weakened structure called bi-adjoints. The concept of an adjunction from 1-category theory consists of two functors and a natural bijection between appropriate hom sets. Mac Lane lists several equivalent ways of describing an adjunction in 39] on pages 79-86. One of these ways involves a universal arrow for each object of the source category. To weaken these concepts, we replace the functors by pseudo functors, the natural bijection of hom sets by a pseudo natural equivalence of categories, and the universal arrow by a bi-universal arrow. The main goal in this section is to prove that a bi-adjunction can be described via pseudo natural equivalences or via bi-universal arrows. This is the meaning of Theorem 8.12 and Theorem 8.13

A close result in the literature can be found in Gray's work [18. His concept of transcendental quasi-adjunction between two 2-functors on page 177 is similar to the concept of bi-adjunction between two pseudo functors except that the functors in a bi-adjoint are allowed to be pseudo. Gray remarks on pages 180-181 that a transcendental quasi-adjunction gives rise to a certain universal mapping property. 
The analogous concept for bi-adjoints is a bi-universal arrow and the appropriate theorem is Theorem 8.12 On page 184 Gray remarks that under certain hypothesis, the universal mapping property gives rise to a quasi-adjunction. The biadjoint version of this is Theorem 8.13 in which the starting functor $G$ is allowed to be a pseudo functor.

Kelly phrases a similar result in 29] on page 316 in terms of homomorphisms of bicategories and birepresentations. His notion of bi-adjoint is the same as in this paper, except that I am considering only pseudo functors between 2-categories rather than homomorphisms between bicategories. Kelly's statement is equivalent to 8.13 after an application of Yoneda's Lemma for bicategories. Yoneda's Lemma for bicategories can be found in [50].

Street makes an observation on page 121 in [50] similar to Theorem 8.13] if each object has a left bilifting then a left bi-adjoint exists. The unit for a left bi-lifting is the bi-universal arrow of Theorem 8.13

MacDonald and Stone also have weakened notion of adjunction in 37] called soft adjunction. In that article they consider strict 2-functors and natural adjunctions between hom sets. They prove theorems about the universality concepts that arise in such a context.

I follow Mac Lane's presentation of adjoints except I account for the 2-cells. The notation in this study is analogous to the notation in Mac Lane's book. Recall the definition of a universal arrow and its uniqueness.

Definition 8.1. Let $S: D \rightarrow C$ be a functor between 1-categories and $c \in O b j C$. Then an object $r \in \operatorname{Obj} D$ and a morphism $u \in \operatorname{Mor}_{C}(c, S r)$ are a universal arrow from c to $S$ if for every $d \in O b j D$ and every $f \in \operatorname{Mor}_{C}(c, S d)$ there exists a unique morphism $f^{\prime} \in M r_{D}(r, d)$ such that $S f^{\prime} \circ u=f$. Pictorially this means for every $d$ and every $f$ as above, there exists a unique $f^{\prime}$ making

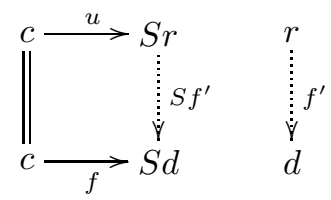

commute. This is equivalent to saying the assignment $f^{\prime} \mapsto S f^{\prime} \circ u$, $M \operatorname{Mr}_{D}(r, d) \rightarrow \operatorname{Mor}_{C}(c, S d)$ is a bijection of hom sets for every fixed $d \in \operatorname{ObjD}$.

Lemma 8.1. Let $u: c \rightarrow S r$ and $u^{\prime}: c \rightarrow S r^{\prime}$ be universal arrows from the object $c$ to the functor $S$. Then there exists a unique morphism $f^{\prime}: r \rightarrow r^{\prime}$ such that $S f^{\prime} \circ u=u^{\prime}$. Moreover, the morphism $f^{\prime}: r \rightarrow r^{\prime}$ is an isomorphism.

Proof: There exist unique morphisms $f^{\prime}$ and $g^{\prime}$ such that the following diagram commutes.

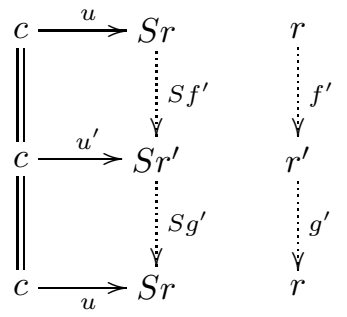


The middle vertical column could be replaced by $S 1_{r}$ to make the outermost rectangle commutative. Hence by the uniqueness we have $g^{\prime} \circ f^{\prime}=1_{r}$. Similarly one shows that $f^{\prime} \circ g^{\prime}=1_{r^{\prime}}$. Hence $f^{\prime}$ is an isomorphism and $S f^{\prime} \circ u=u^{\prime}$.

Before weakening the concept of universal arrow, I prove a simple lemma that will make it easier to visualize a bi-universal arrow.

Lemma 8.2. Let $X \underset{\psi}{\stackrel{\phi}{\rightleftarrows}} A$ be adjoint functors with unit $\theta: 1_{X} \Rightarrow \psi \circ \phi$ and counit $\mu: \phi \circ \psi \Rightarrow 1_{A}$. Suppose that both the unit and the counit are natural isomorphisms. Let $\nu: \phi(x) \rightarrow a$ be a morphism in $A$ and $x \in \operatorname{Obj} X, a \in O b j A$. Then there exists a unique morphism $\nu^{\prime}: x \rightarrow \psi($ a) such that

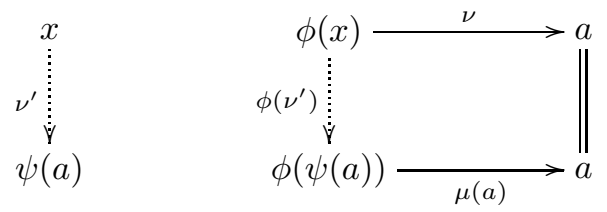

commutes. Moreover, $\nu^{\prime}$ is iso if and only if $\nu$ is iso.

Proof: The existence and uniqueness claims follow because $\mu(a)$ is a universal arrow from $\phi$ to $a$. If $\nu^{\prime}$ is iso, then $\phi\left(\nu^{\prime}\right)$ is iso and so is $\nu=\mu(a) \circ \phi\left(\nu^{\prime}\right)$ because $\mu(a)$ is iso by hypothesis. It only remains to show that $\nu^{\prime}$ is iso if $\nu$ is iso. Suppose $\nu$ is iso. Then $\phi\left(\nu^{\prime}\right)$ is iso from the commutivity of the diagram because $\mu(a)$ and $\nu$ are iso. By the naturality of $\theta$ we have

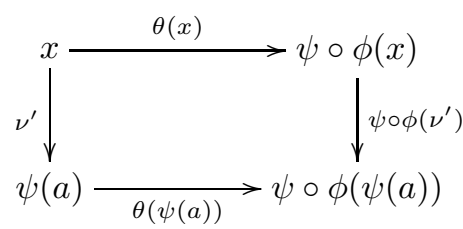

commutes. Then $\nu^{\prime}$ is iso because $\theta(x), \theta(\psi(a))$, and $\psi\left(\phi\left(\nu^{\prime}\right)\right)$ are iso.

To weaken the concept of universal arrow in the context of 2-categories, we replace the bijection of sets above by an equivalence of the appropriate morphism categories.

Definition 8.2. Let $S: \mathcal{D} \rightarrow \mathcal{C}$ be a pseudo functor between 2-categories and $C \in O b j \mathcal{C}$. Then an object $R \in O b j \mathcal{D}$ and a morphism $u \in \operatorname{Mor}_{\mathcal{C}}(C, S R)$ are a $b i$ universal arrow from $C$ to $S$ if for every $D \in O \operatorname{Obj} \mathcal{D}$ the functor $\phi: \operatorname{Mor}_{\mathcal{D}}(R, D) \rightarrow$ $\operatorname{Mor}_{\mathcal{C}}(C, S D)$ defined by $f^{\prime} \mapsto S f^{\prime} \circ u$ and $\gamma \mapsto S \gamma * i_{u}$ is an equivalence of categories.

We suppressed the dependence of $\phi$ on $D$ in the notation of the definition. This definition implies that $\phi$ has a right adjoint $\psi$ such that the counit $\mu: \phi \circ \psi \Rightarrow$ $1_{M o r_{C}(C, S D)}$ and unit are natural isomorphisms. Pictorially the definition implies that for every object $D \in \operatorname{Obj} \mathcal{D}$ and every morphism $f: C \rightarrow S D$ in $\mathcal{C}$ there exists an $f^{\prime}$ and a natural universal 2-cell $\mu(f)$ which is iso (an arrow of the counit) as in 
the following diagram.

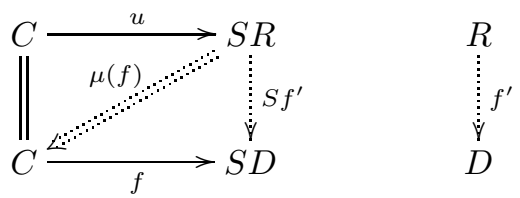

The assignment $\psi: f \mapsto f^{\prime}$ is functorial and $\mu: \phi \circ \psi \Rightarrow 1_{M o r_{C}(C, S D)}$ is a natural transformation. This diagram is not equivalent to the definition because it does not express the naturality of the 2-cells, nor does it include the natural isomorphism (the unit) from the the identity functor on $\operatorname{Mor}_{\mathcal{D}}(R, D)$ to $\psi \circ \phi$. The universality of the 2-cell $\mu(f)$ from the functor $\phi$ to the object $f$ means pictorially that the arrow $f^{\prime}$ is unique up to 2 -cell in the following way. If $\bar{f}^{\prime}: R \rightarrow D$ is an arrow in $\mathcal{D}$ and $\nu$ is a (not necessarily iso) 2 -cell as in

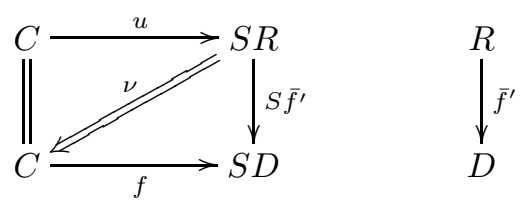

then there exists a unique 2-cell $\nu^{\prime}: \bar{f}^{\prime} \Rightarrow f^{\prime}$ whose $\phi$ image factors $\nu$ via the universal arrow $\mu(f)$, i.e. $\nu^{\prime}$ is such that

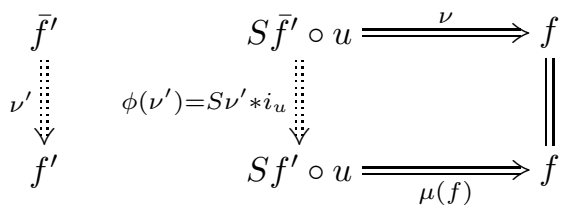

commutes. We also know that $\nu^{\prime}$ is iso if and only if $\nu$ is iso as in Lemma 8.2 Note that these diagrams are dual to Definition 8.1 although it is the same concept of universal arrow.

One can ask if the equivalences of categories in the definition of bi-universal arrow can be chosen in some natural way as in Remark 2.3. They can in fact as the following theorem shows.

Theorem 8.3. Let $u: C \rightarrow S R$ be a bi-universal arrow from $C$ to the pseudo functor $S$ as in Definition 8.2. Let $\phi_{D}: \operatorname{Mor}_{\mathcal{D}}(R, D) \rightarrow \operatorname{Mor}_{\mathcal{C}}(C, S D)$ be the functor defined by $f^{\prime} \mapsto S f^{\prime} \circ u$ and $\gamma \mapsto S \gamma * i_{u}$. Then $D \mapsto \phi_{D}$ is a pseudo natural transformation $\operatorname{Mor}_{\mathcal{D}}(R,-) \Rightarrow \operatorname{Mor}_{\mathcal{C}}(C, S-)$. For $D \in \operatorname{Obj\mathcal {D}}$ let $\psi_{D}$ : $\operatorname{Mor}_{\mathcal{C}}(C, S D) \rightarrow \operatorname{Mor}_{\mathcal{D}}(R, D)$ be a right adjoint to $\phi_{D}$ such that the unit $\eta_{D}:$ $1_{M o r_{\mathcal{D}}(R, D)} \Rightarrow \psi_{D} \circ \phi_{D}$ and the counit $\varepsilon_{D}: \phi_{D} \circ \psi_{D} \Rightarrow 1_{M o r_{\mathcal{C}}(C, S D)}$ are natural isomorphisms. Then $D \mapsto \psi_{D}$ is a pseudo natural transformation and $D \mapsto \eta_{D}$ and $D \mapsto \varepsilon_{D}$ are iso modifications $i_{M o r_{\mathcal{D}}(R,-)} \rightsquigarrow \psi \odot \phi$ and $\phi \odot \psi \rightsquigarrow i_{M o r_{\mathcal{C}}\left(C, S_{-}\right)}$which satisfy the triangle identities.

Proof: $\quad$ Let $F, G: \mathcal{D} \rightarrow C a t$ be the pseudo functors defined by $F(D)=$ $\operatorname{Mor}_{\mathcal{D}}(R, D)$ and $G(D)=\operatorname{Mor}_{\mathcal{C}}(C, S D)$. Then $F$ is a strict 2-functor. One can prove that $\phi: F \Rightarrow G$ is a pseudo natural transformation by defining the coherence 2-cell $\tau$ in terms of $\gamma^{S}$ and then using the unit and composition axioms for $S$ to prove the unit and composition axioms for $\phi$. After doing that, we are in the setup of Lemma 8.5 from which everything else follows. 
In analogy to the uniqueness statement for universal arrows, we have a uniqueness statement for bi-universal arrows. It requires the concept of pseudo isomorphism in a 2-category.

Definition 8.3. Let $\mathcal{D}$ be a 2-category and $f: R \rightarrow R^{\prime}$ a morphism in $\mathcal{D}$. Then $f$ is a pseudo isomorphism if there exists a morphism $g: R^{\prime} \rightarrow R$ and iso 2-cells $g \circ f \Rightarrow 1_{R}$ and $g \circ f \Rightarrow 1_{R^{\prime}}$. A pseudo isomorphism is also called an equivalence.

Lemma 8.4. Let $S: \mathcal{D} \rightarrow \mathcal{C}$ be a pseudo functor. Let $u_{1}: C \rightarrow S R_{1}$ and $u_{2}: C \rightarrow$ $S R_{2}$ be bi-universal arrows from $C$ to $S$. Then there exists a pseudo isomorphism $g^{\prime}: R_{1} \rightarrow R_{2}$ in $\mathcal{D}$ and an iso 2-cell as in (11).
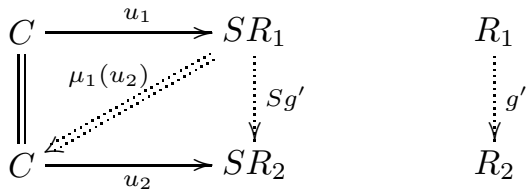

Morever, if $\bar{g}^{\prime}$ and $\nu$ are a morphism and an iso 2-cell that also fill in the diagram, then $\bar{g}^{\prime}$ and $g^{\prime}$ are isomorphic via the unique 2-cell $\nu^{\prime}: \bar{g}^{\prime} \rightarrow g^{\prime}$ such that $\mu_{1}\left(u_{2}\right) \circ$ $\left(S \nu^{\prime} * i_{u_{1}}\right)=\nu$.

Proof: The bi-universality of $u_{1}$ and $u_{2}$ guarantees the existence of arrows $f^{\prime}, g^{\prime}$, and $h^{\prime}$ and iso 2-cells $\mu_{1}\left(u_{2}\right), \mu_{2}\left(u_{1}\right)$, and $\mu_{1}\left(u_{1}\right)$ to fill in the following diagrams.
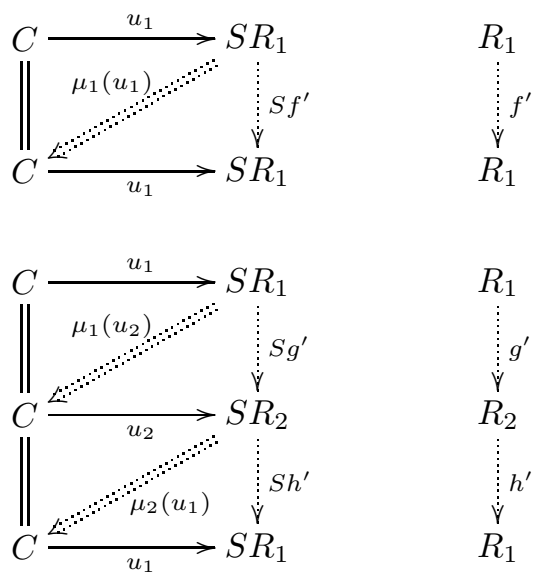

The arrow $1_{R_{1}}$ also fills in the diagram
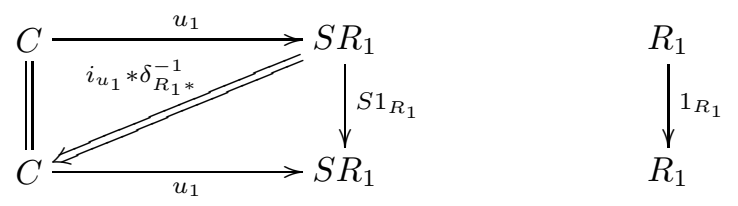

with an iso 2-cell. Diagram (13) combined appropriately with $\left(\gamma_{g^{\prime}, h^{\prime}}^{S}\right)^{-1}$ gives an iso 2-cell $h^{\prime} \circ g^{\prime} \Rightarrow f^{\prime}$ by the comments after the definition of bi-universal arrow. Similarly, diagram (14) gives an iso 2 -cell $1_{R_{1}} \Rightarrow f^{\prime}$ for the same reason. Combining these two iso 2-cells appropriately gives an iso 2-cell $h^{\prime} \circ g^{\prime} \Rightarrow 1_{R_{1}}$. By a similar argument one obtains an iso 2-cell $g^{\prime} \circ h^{\prime} \Rightarrow 1_{R_{2}}$. Thus $g^{\prime}: R_{1} \rightarrow R_{2}$ is a pseudo 
isomorphism. The iso 2-cell between $\bar{g}^{\prime}$ and $g^{\prime}$ is also guaranteed by the comments after the definition of bi-universal arrow in 8.2

After these preparations involving bi-universal arrows, we can now introduce the main concept of this section.

Definition 8.4. Let $\mathcal{X}$ and $\mathcal{A}$ be 2-categories. A bi-adjunction $\langle F, G, \phi\rangle: \mathcal{X} \rightarrow \mathcal{A}$ consists of the following data

- Pseudo functors

$$
\mathcal{X} \underset{G}{\stackrel{F}{\rightleftarrows}} \mathcal{A}
$$

between 2-categories

- For all $X \in O b j \mathcal{X}$ and all $A \in O b j \mathcal{A}$ an equivalence of categories $\phi_{X, A}$ : $\operatorname{Mor}_{\mathcal{A}}(F X, A) \rightarrow \operatorname{Mor}_{\mathcal{X}}(X, G A)$ assigned in such a way to make $\phi$ into a pseudo natural transformation in each variable between the following pseudo functors of two variables.

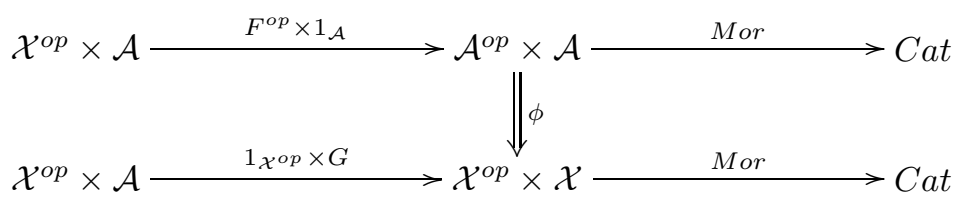

In this situation, $F$ is called a left bi-adjoint for $G$ and $G$ is called a right bi-adjoint for $F$.

Recall again that a bi-adjoint is called a lax adjoint in 24, 25], 26. The degree of uniqueness of a left bi-adjoint (if a left bi-adjoint exists), will be dealt with at the end of this section. One can ask whether or not an adjoint functor $\psi_{X, A}: \operatorname{Mor}_{\mathcal{X}}(X, G A) \rightarrow \operatorname{Mor}_{\mathcal{A}}(F X, A)$ to $\phi_{X, A}$ can be chosen in a natural way. This is similar to the question answered in Remark 2.3 for bicolimits. To show that right adjoints can be chosen in a pseudo natural way, I need the following lemma.

Lemma 8.5. Let $F, G: \mathcal{A} \rightarrow$ Cat be pseudo functors and $F$ a strict 2-functor. Suppose we have a pseudo natural transformation $\phi: F \Rightarrow G$ such that $\phi_{A}: F A \rightarrow$ $G A$ is an equivalence of categories for all $A \in O b j \mathcal{A}$. For each $A \in O b j \mathcal{A}$, let $\psi_{A}: G A \rightarrow F A$ be a right adjoint to $\phi_{A}$ such that the unit $\eta_{A}: 1_{F A} \Rightarrow \psi_{A} \circ \phi_{A}$ and counit $\varepsilon_{A}: \phi_{A} \circ \psi_{A} \Rightarrow 1_{G A}$ are natural isomorphisms. Then $A \mapsto \psi_{A}$ is a pseudo natural transformation $G \Rightarrow F$ and $A \mapsto \eta_{A}$ respectively $A \mapsto \varepsilon_{A}$ define iso modifications $\eta: i_{F} \rightsquigarrow \psi \odot \phi$ respectively $\varepsilon: \phi \odot \psi \rightsquigarrow i_{G}$. Furthermore, $\eta$ and $\varepsilon$ satisfy the triangle identities.

Proof: $\quad$ For all $A \in O b j \mathcal{A}$ there exists such a right adjoint $\psi_{A}$ because $\phi_{A}$ is an equivalence of categories.

To show that $A \mapsto \psi_{A}$ is a pseudo natural transformation, we need to define the coherence 2-cell $\tau_{f}^{\prime}$ for each morphism $f$ of $\mathcal{A}$, show that it is natural, it satisfies the unit axiom, and that it satisfies the composition axiom.

For a morphism $f: A \rightarrow B$ in $\mathcal{A}$ let $\tau_{f}: G f \circ \phi_{A} \Rightarrow \phi_{B} \circ F f$ denote the coherence 2 -cell belonging to the pseudo natural transformation $\phi$. Define $\tau_{f}^{\prime}: F f \circ \psi_{A} \Rightarrow$ 
$\psi_{B} \circ G f$ to be the composition of the 2-cells in the following diagram.

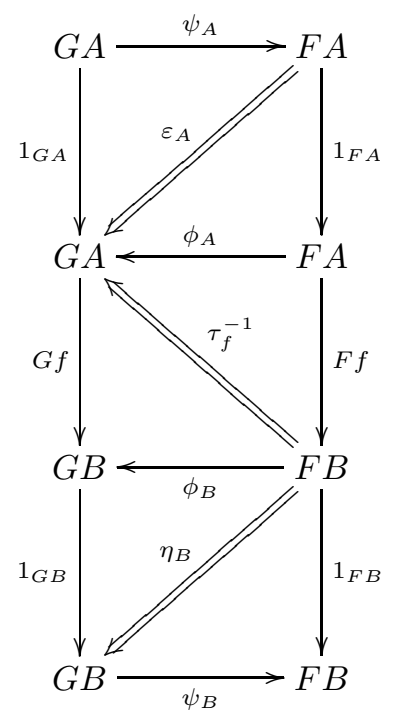

I claim that the assignment $f \mapsto \tau_{f}^{\prime}$ is natural in $f$. To see this, let $f, g: A \rightarrow B$ be morphisms in $\mathcal{A}$ and $\mu: f \Rightarrow g$ a 2-cell in $\mathcal{A}$. Then $\tau_{f}^{\prime}$ is the composition of top row of 2-cells in the following diagram and $\tau_{g}^{\prime}$ is the bottom composition.

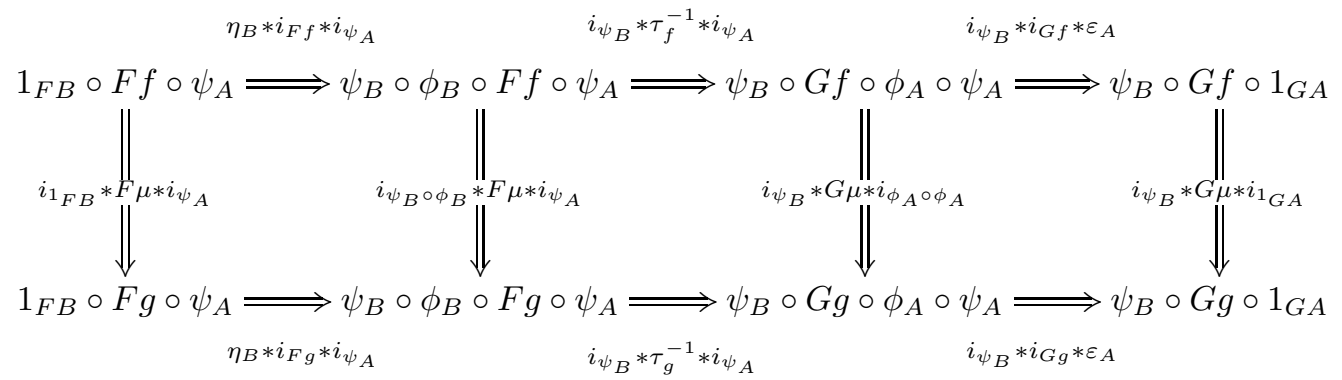

The left square and the right square commute because of the interchange law and the defining property of identity 2-cells. The middle square commutes because $f \mapsto \tau_{f}$ is natural by the definition of $\phi$ pseudo natural. Hence the outermost rectangle commutes and $f \mapsto \tau_{f}^{\prime}$ is natural.

I claim that $\tau^{\prime}$ satisfies the unit axiom for pseudo natural transformations. Since $F$ is strict, proving the coherence diagram reduces to proving that $\tau_{1_{A}}^{\prime}=i_{\psi_{A}} * \delta_{A *}^{G}$. 
Using the definition of $\tau^{\prime}$ above and the unit axiom for $\tau$ we see that $\tau_{1_{A}}^{\prime}$ is the composition of 2-cells in diagram (16).

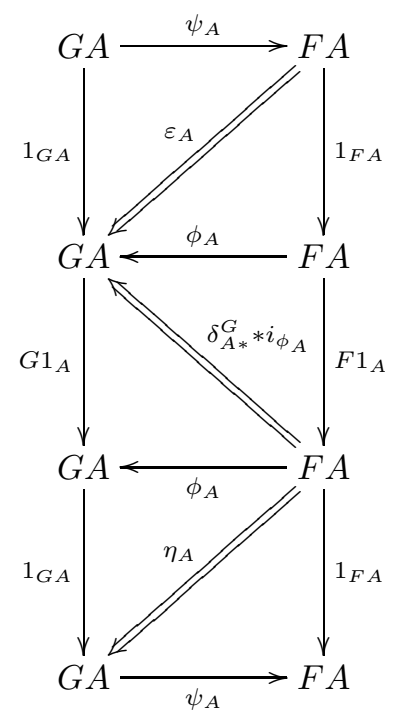

But the composition of 2-cells in (16) is the same as the composition of 2-cells in (17) by the interchange law.

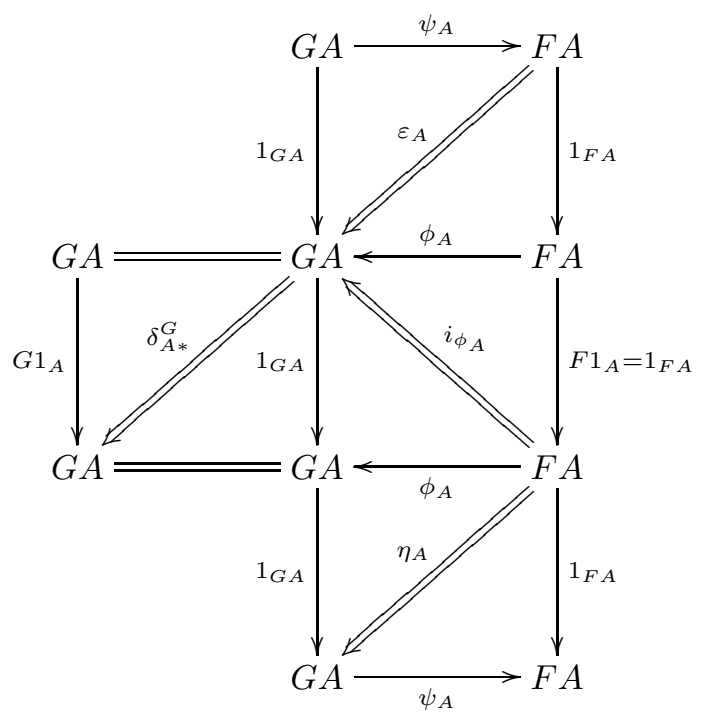

By one of the triangle identities we see that the right three squares of (17) collapse to $i_{\psi_{A}}$ and therefore (16) is the same as $i_{\psi_{A}} * \delta_{A *}^{G}$. Hence $\tau_{1_{A}}^{\prime}=i_{\psi_{A}} * \delta_{A *}^{G}$ and the unit axiom is satisfied.

I claim that $\tau^{\prime}$ satisfies the composition axiom for pseudo natural transformations. Let $A \stackrel{f}{\longrightarrow} B \stackrel{g}{\longrightarrow} C$ be morphisms in $\mathcal{A}$. Since $F$ is a strict 2-functor, proving the composition coherence reduces to proving that 
$\tau_{g \circ f}^{\prime}=\left(i_{\psi_{C}} * \gamma_{f, g}^{G}\right) \odot\left(\tau_{g}^{\prime} * i_{G f}\right) \odot\left(i_{F g} * \tau_{f}^{\prime}\right)$. Following the same approach as for the unit axiom, we write out $\tau_{g \circ f}^{\prime}$ in (18).

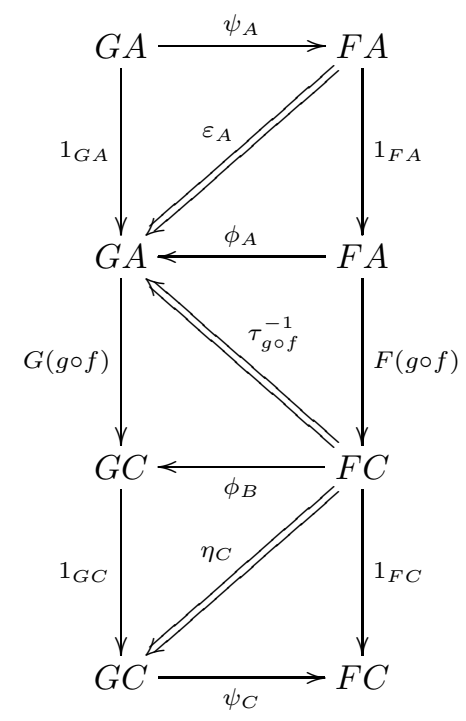

Using the composition axiom for $\tau$ and writing the 2-cells more compactly we see that the composition of 2-cells in diagram (18) is the same as in diagram (19).

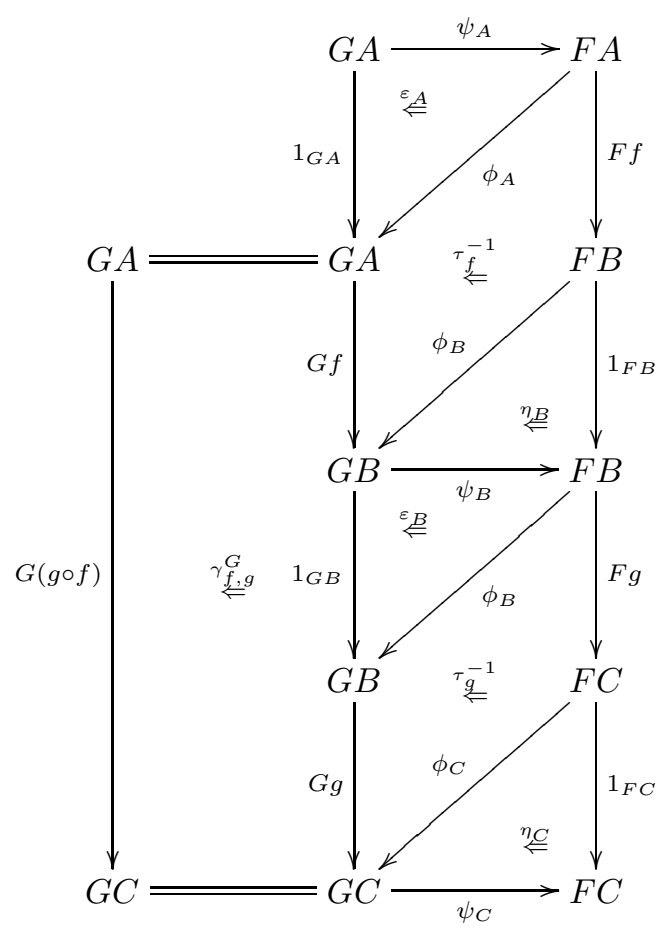


The middle parallelogram involving $\eta_{B}$ and $\varepsilon_{B}$ is the same as $i_{\phi_{B}}$ by the triangle identity. Hence (19) is $\left(i_{\psi_{C}} * \gamma_{f, g}^{G}\right) \odot\left(\tau_{g}^{\prime} * i_{G f}\right) \odot\left(i_{F g} * \tau_{f}^{\prime}\right)$ and we conclude that $\tau_{g \circ f}^{\prime}=\left(i_{\psi_{C}} * \gamma_{f, g}^{G}\right) \odot\left(\tau_{g}^{\prime} * i_{G f}\right) \odot\left(i_{F g} * \tau_{f}^{\prime}\right)$ as required by the composition axiom.

Thus far we have shown that $A \mapsto \psi_{A}$ is a pseudo natural transformation $G \Rightarrow F$. Next I show that $A \mapsto \eta_{A}$ defines a modification $i_{F} \rightsquigarrow \psi \odot \phi$.

Let $f, g: A \rightarrow B$ be morphisms in the 2-category $\mathcal{A}$ and $\gamma: f \Rightarrow g$ a 2-cell. I claim that the compositions in diagrams (11) and (2) are the same, i.e. that $\eta$ is a modification. Our diagrams will of course have $F=G, \alpha=i_{F}, \beta=\psi \odot \phi$, and the coherence iso belonging to $i_{F}$ is trivial while the coherence iso for the composite pseudo natural transformation $\psi \odot \phi$ is $\left(i_{\psi_{B}} * \tau_{f}\right) \odot\left(\tau_{f}^{\prime} * i_{\phi_{A}}\right)$ by the remarks on page[ about coherence isos for a vertical composition of pseudo natural transformations. Then we see that the composition (2) is $\eta_{B} * F \gamma$. I proceed by reducing (11) to $\eta_{B} * F \gamma$. The composition in diagram (11) is explicitly (20), where I left off the vertical equal signs.

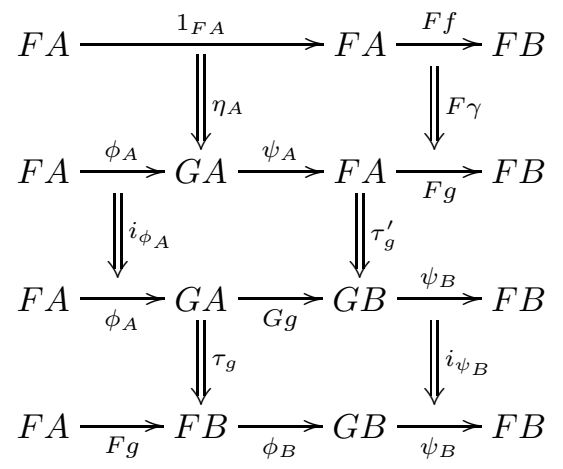

Writing out the definition $\tau_{g}^{\prime}$ in (20) and including some identities gives (21).

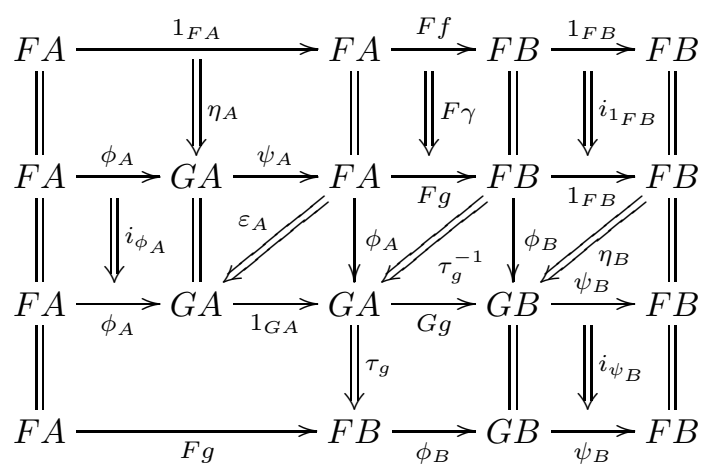

After cancelling $\tau_{g}$ with $\tau_{g}^{-1}$ and using one of the triangle identities we see that (21) is the same as $\eta_{B} * F \gamma$. Thus we conclude that (1) is the same as (2) and that $A \mapsto \eta_{A}$ is a modification.

One can similarly show that $A \mapsto \varepsilon_{A}$ is a modification.

The modifications $\eta$ and $\varepsilon$ satisfy the triangle identities because the individual 2-cells $\eta_{A}$ and $\varepsilon_{A}$ do. 
Now I use this lemma to prove how the right adjoints $\psi_{X, A}: \operatorname{Mor}_{\mathcal{X}}(X, G A) \rightarrow$ $\operatorname{Mor}_{\mathcal{A}}(F X, A)$ to $\phi_{X, A}$ can be chosen in a pseudo natural way in the following theorem.

Theorem 8.6. Let $\langle F, G, \phi\rangle: \mathcal{X} \rightarrow \mathcal{A}$ be a bi-adjunction. For all $X \in \operatorname{Obj\mathcal {X}}$ and all $A \in \operatorname{Obj\mathcal {A}}_{\mathcal{A}}$ let $\psi_{X, A}: \operatorname{Mor}_{\mathcal{X}}(X, G A) \rightarrow \operatorname{Mor}_{\mathcal{A}}(F X, A)$ be a right adjoint to $\phi_{X, A}$ such that the unit $\eta_{X, A}: 1_{M o r_{A}(F X, A)} \Rightarrow \psi_{X, A} \circ \phi_{X, A}$ and the counit $\varepsilon_{X, A}: \phi_{X, A} \circ \psi_{X, A} \Rightarrow 1_{\operatorname{Mor}_{\mathcal{X}}(X, G A)}$ are natural isomorphisms. Then the assignment $(X, A) \mapsto \psi_{X, A}$ is pseudo natural in each variable. Moreover, the assignments $(X, A) \mapsto \eta_{X, A}$ and $(X, A) \mapsto \varepsilon_{X, A}$ comprise modifications in each variable of the form $\eta: i_{M_{\text {or }}(F-,-)} \rightsquigarrow \psi \odot \phi$ and $\varepsilon: \phi \odot \psi \rightsquigarrow i_{M_{\mathcal{X}}(-, G-)}$.

Proof: I prove the pseudo naturality and modification in the second variable. The first variable is similar. Let $\bar{F}$ respectively $\bar{G}$ be the pseudo functor $\mathcal{A} \rightarrow$ Cat obtained by holding $X$ fixed in the first respectively second row in Definition 8.4 See the proof of Lemma 8.11 for a precise description of $\bar{F}$ and $\bar{G}$. The pseudo functor $\bar{F}$ is actually a strict 2-functor because it is the composition of strict 2functors. If we drop the notation $X$ in all occurences, we see that we are precisely in the setup of Lemma 8.5. This proves the theorem for the second variable. To prove it for the first variable one only needs to prove an analogue of Lemma 8.5 for $F$ pseudo and $G$ strict.

Next I prove a series of lemmas needed to prove Theorems 8.12 and 8.13

Lemma 8.7. Let $\mathcal{X}$ and $\mathcal{A}$ be 2-categories. Let $\langle F, G, \phi\rangle: \mathcal{X} \rightarrow \mathcal{A}$ be a bi-adjunction and let $\eta_{X}:=\phi_{X, F X}\left(1_{F X}\right): X \rightarrow G F X$. Then $\eta_{X}: X \rightarrow G(F X)$ is a bi-universal arrow from $X$ to $G$.

Proof: The assignment $(X, A) \mapsto \phi_{X, A}$ is pseudo natural in each variable by assumption. Let $\tau$ denote the coherence 2-cells for $\phi_{X,-}$. From the definition of pseudo natural transformation $\phi_{X,-}$ we obtain for $f^{\prime} \in \operatorname{Mor}_{\mathcal{A}}(F X, D)$ the following diagram in Cat.

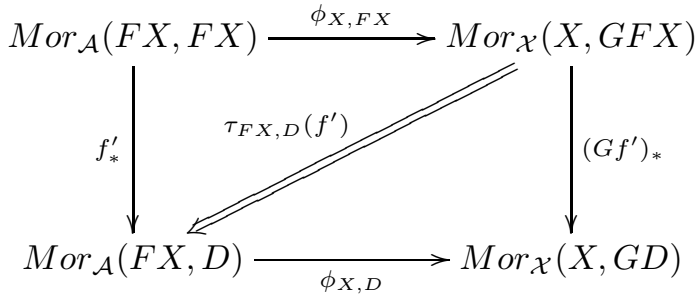

Chasing $1_{F X}$ along this diagram gives a diagram in the 2-category $\mathcal{X}$.

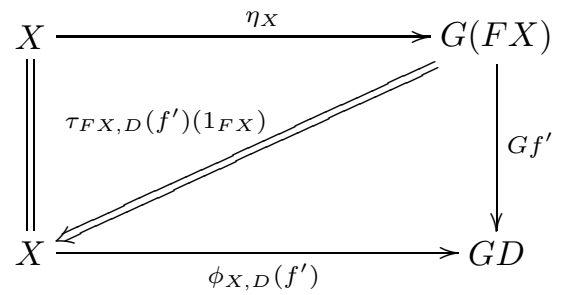

The map $\operatorname{Mor}_{\mathcal{A}}(F X, D) \ni f^{\prime} \mapsto \tau_{F X, D}\left(f^{\prime}\right)\left(1_{F X}\right)$ is natural. This fact combined with the diagram in $\mathcal{X}$ above says that we have a natural isomorphism from the 
functor $\operatorname{Mor}_{\mathcal{A}}(F X, D) \ni f^{\prime} \mapsto G f^{\prime} \circ \eta_{X} \in \operatorname{Mor}_{\mathcal{X}}(X, G D)$ to the functor $f^{\prime} \mapsto$ $\phi_{X, D}\left(f^{\prime}\right)$. From the definition of bi-adjunction, $\phi_{X, D}$ is an equivalence of categories. Hence $f^{\prime} \mapsto G f^{\prime} \circ \eta_{X}$ is naturally isomorphic to an equivalence of categories and is therefore itself an equivalence of categories $\operatorname{Mor}_{\mathcal{A}}(F X, D) \rightarrow \operatorname{Mor}_{\mathcal{X}}(X, G D)$. We conclude that $\eta_{X}$ is a bi-universal arrow.

Lemma 8.8. Let $\mathcal{X}$ and $\mathcal{A}$ be 2-categories. Let $\langle F, G, \phi\rangle: \mathcal{X} \rightarrow \mathcal{A}$ be a bi-adjunction and let $\eta_{X}:=\phi_{X, F X}\left(1_{F X}\right): X \rightarrow G F X$. Then the assignment $X \mapsto \eta_{X}$ is a pseudo natural transformation $1_{\mathcal{X}} \Rightarrow G F$.

Proof: $\quad$ Let $f: X^{\prime} \rightarrow X$ be a morphism of $\mathcal{X}$. Let $\tau$ respectively $\tau^{\prime}$ denote the coherence 2-cells for the pseudo natural transformation $\phi_{X^{\prime},-}$ respectively $\phi_{-, F X}$. I must show that we have a 2 -cell

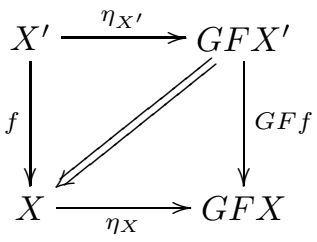

in $\mathcal{X}$ which is natural in $f$ and satisfies the coherences involving $\delta$ and $\gamma$. Since $\phi$ is pseudo natural in each variable we have the diagram

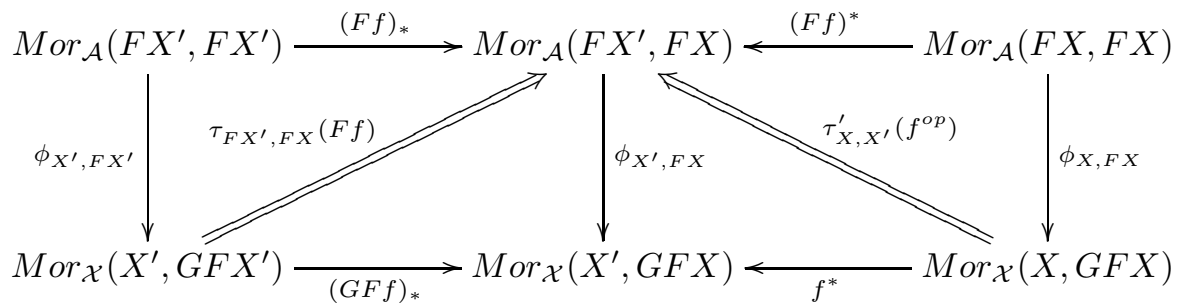

in Cat. By chasing $1_{F X^{\prime}}$ and $1_{F X}$ from the upper corners of this diagram to the center and then down we see that they both get mapped to $\phi_{X^{\prime}, F X}(F f)$. Chasing the identities in the opposite directions and evaluating the natural transformations at the identities yields a diagram of 2-cells in $\mathcal{X}$.

$$
(G F f) \circ \eta_{X^{\prime}} \stackrel{\tau_{F X^{\prime}, F X}(F f)\left(1_{F X^{\prime}}\right)}{\Longrightarrow} \phi_{X^{\prime}, F X}(F f) \stackrel{\tau_{X, X^{\prime}}^{\prime}\left(f^{o p}\right)\left(1_{F X}\right)}{=} \eta_{X} \circ f
$$

These 2-cells are invertible by hypothesis. Let $\tilde{\tau}_{X^{\prime}, X}(f)$ denote the composition from left to right obtained by inverting the second 2-cell. $\tilde{\tau}_{X^{\prime}, X}$ is natural in $f$ because the constituents are natural in $f$. The coherence 2-cells $\tilde{\tau}$ satisfy the coherences with $\delta$ and $\gamma$ from $G F$ also because the individual constituents do. Hence

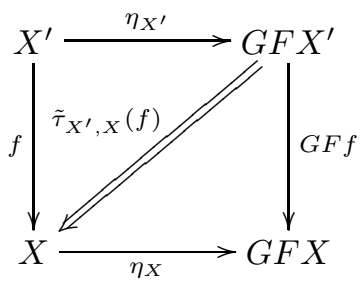


is natural in $f$ and satisfies the required coherences, so $X \mapsto \eta_{X}$ is a pseudo natural transformation.

Thus we have seen that given a bi-adjunction $\phi$ we get a pseudo natural transformation $\eta$ whose arrows are bi-universal arrows. Now we consider the converse of this statement.

Lemma 8.9. Let $\mathcal{X}$ and $\mathcal{A}$ be 2-categories. Let $\mathcal{X} \underset{G}{\stackrel{F}{\rightleftarrows}} \mathcal{A}$ be pseudo functors between 2-categories. Let $\eta: 1_{\mathcal{X}} \Rightarrow G F$ be a pseudo natural transformation such that each arrow $\eta_{X}: X \rightarrow G(F X)$ is a bi-universal arrow from $X$ to $G$. Define $\phi_{X, A}(f):=G f \circ \eta_{X}$ for each $f: F X \rightarrow A$ and $\phi_{X, A}(\gamma):=G \gamma * i_{\eta_{X}}$ for each $\gamma: f \Rightarrow f^{\prime}$. Then $\phi_{X, A}: \operatorname{Mor}_{\mathcal{A}}(F X, A) \rightarrow \operatorname{Mor}_{\mathcal{X}}(X, G A)$ is an equivalence of categories for all $X \in \operatorname{Obj\mathcal {X}}$ and all $A \in \operatorname{Obj} \mathcal{A}$.

Proof: The functor $\phi_{X, A}$ is an equivalence since $\eta_{X}$ is a bi-universal arrow.

Lemma 8.10. Let $\mathcal{X}$ and $\mathcal{A}$ be 2-categories. Let $\mathcal{X} \underset{G}{\stackrel{F}{\rightleftarrows}} \mathcal{A}$ be pseudo functors between 2-categories. Let $\eta: 1_{\mathcal{X}} \Rightarrow G F$ be a pseudo natural transformation such that each $\eta_{X}: X \rightarrow G(F X)$ is a bi-universal arrow from $X$ to $G$. Let $\phi_{X, A}$ be defined as in Lemma 8.9 above. Then for fixed $A \in O b j \mathcal{A}$ the assignment $O b j \mathcal{X}^{o p} \ni$ $X \mapsto \phi_{X, A}$ denoted $\phi_{-, A}$ is pseudo natural.

Proof: $\quad$ Let $A \in O b j \mathcal{A}$ be a fixed object throughout this proof. Let $\bar{F}: \mathcal{X}^{o p} \rightarrow$ Cat denote the pseudo functor obtained by holding $A$ fixed in the top row in the definition of bi-adjunction. This means $\bar{F}(X)=\operatorname{Mor}_{\mathcal{A}}(F X, A), \bar{F}\left(f^{o p}\right)=$ $(F f)^{*}$, and for $\alpha: f^{o p} \Rightarrow\left(f^{\prime}\right)^{o p}$ in $\mathcal{X}$ the natural transformation $\bar{F}(\alpha):(F f)^{*} \Rightarrow$ $\left(F f^{\prime}\right)^{*}$ is $h \mapsto i_{h} * F \alpha$. Note that the morphisms of $\mathcal{X}^{o p}$ are formally the opposites of morphisms of $\mathcal{X}$, but the 2-cells of $\mathcal{X}^{o p}$ are precisely the same as the 2-cells in $\mathcal{X}$. The vertical composition is the same in both $\mathcal{X}^{o p}$ and $\mathcal{X}$, although the horizontal compositions are switched. The pseudo functor $\bar{F}$ is the composition of a pseudo functor and a strict functor. For morphisms $X \stackrel{f}{\longrightarrow} Y \stackrel{g}{\longrightarrow} Z$ in $\mathcal{X}$ we have $\gamma_{g^{o p}, f^{o p}}^{\bar{F}}: h \mapsto i_{h} * \gamma_{f, g}^{F}$ and for $X \in O b j \mathcal{X}^{o p}$ we have $\delta_{X *}^{\bar{F}}: h \mapsto i_{h} * \delta_{X *}^{F}$ by the rules for composition of pseudo functors. Then $\gamma_{g^{o p}, f^{o p}}^{\bar{F}}: \bar{F}\left(f^{o p}\right) \circ \bar{F}\left(g^{o p}\right) \Rightarrow$ $\bar{F}\left(f^{o p} \circ g^{o p}\right)$ and $\delta_{X *}^{\bar{F}}: 1_{\bar{F} X} \Rightarrow \bar{F}\left(1_{X}\right)$. Let $\bar{G}$ denote the strict 2-functor obtained by holding $A$ fixed in the bottom row in the definition of bi-adjunction. This means $\bar{G}(X)=\operatorname{Mor}_{\mathcal{X}}(X, G A), \bar{G}\left(f^{o p}\right)=f^{*}$, and for $\alpha: f^{o p} \Rightarrow\left(f^{\prime}\right)^{o p}$ in $\mathcal{X}$ the natural transformation $\bar{G}(\alpha): \bar{G}\left(f^{o p}\right) \Rightarrow \bar{G}\left(\left(f^{\prime}\right)^{o p}\right)$ is the natural transformation $h \mapsto i_{h} * \alpha$. The 2 -functor $\bar{G}$ is the composition of two strict 2 -functors and is therefore strict.

In order to prove that $\phi_{-, A}$ is a pseudo natural transformation from $\bar{F}$ to $\bar{G}$ one must display coherence 2-cells $\tau^{\prime}$ up to which $\phi_{-, A}$ is natural and prove that they satisfy the coherences involving $\delta$ and $\gamma$. Now I describe this $\tau^{\prime}$ and later prove the coherences. Let $\tilde{\tau}$ denote the coherence 2-cells which make $\eta: 1_{\mathcal{X}} \Rightarrow G F$ pseudo 
natural, i.e. for all $f: X \rightarrow Y$ in $\mathcal{X}$ we have

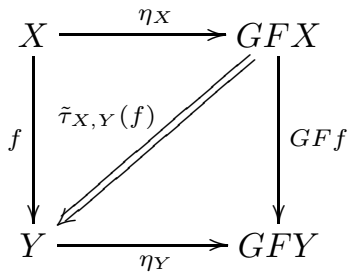

in $\mathcal{X}$. Define a natural isomorphism $\tau_{f^{o p}}^{\prime}=\tau_{Y, X}^{\prime}\left(f^{o p}\right): \bar{G}\left(f^{o p}\right) \circ \phi_{Y, A} \Rightarrow \phi_{X, A} \circ$ $\bar{F}\left(f^{o p}\right)$ by $h \mapsto\left(\gamma_{F f, h}^{G} * i_{\eta_{X}}\right) \odot\left(i_{G h} *\left(\tilde{\tau}_{X, Y}(f)\right)^{-1}\right)$ for $h \in \operatorname{Mor}_{\mathcal{A}}(F Y, A)$ as in the following diagram.

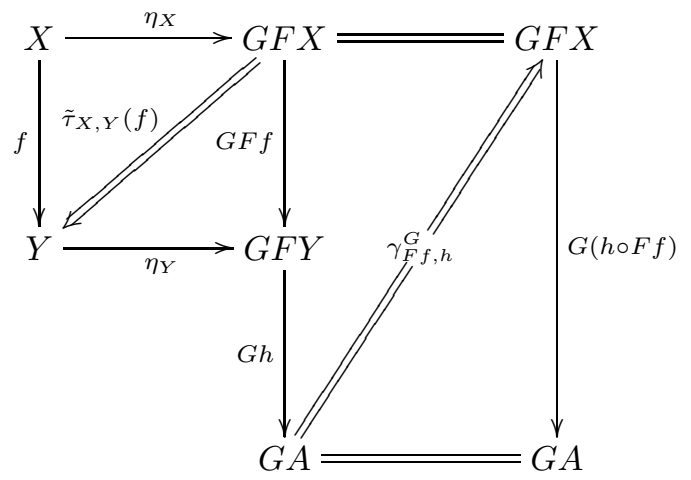

The map $\tau_{Y, X}^{\prime}\left(f^{o p}\right)$ is a natural transformation because $\gamma_{F f, h}^{G}$ is natural in $h$. The assignment $f^{o p} \mapsto \tau_{Y, X}^{\prime}\left(f^{o p}\right)$ is also natural for a similar reason.

I claim that $\tau^{\prime}$ satisfies the unit axiom for pseudo natural transformations. I must show that the diagram of 2-cells in Cat

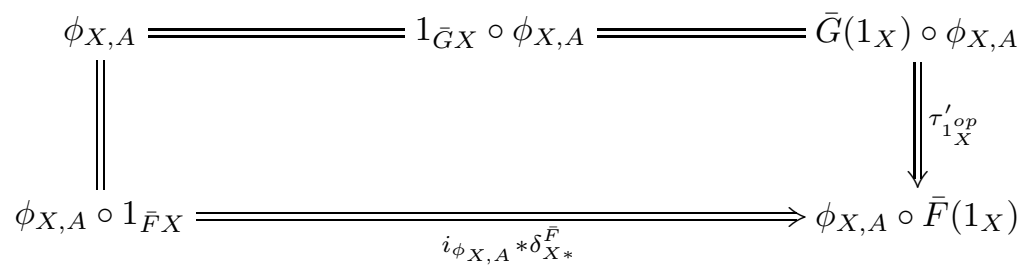

commutes for all $X \in O b j \mathcal{X}$. Evaluating this diagram on a morphism $h: F X \rightarrow A$ of $\mathcal{A}$ gives the diagram of 2 -cells

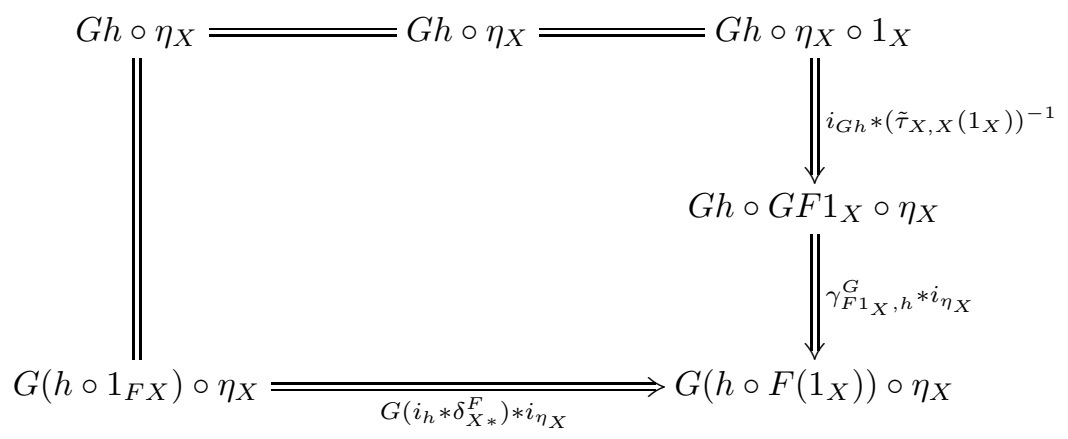


in $\mathcal{X}$. Since $\eta: 1_{\mathcal{X}} \Rightarrow G F$ is a pseudo natural transformation from the strict 2functor to the composition $G \circ F$ of pseudo functors, its unit axiom for $\tilde{\tau}$ simplifies to the following commutative diagram.

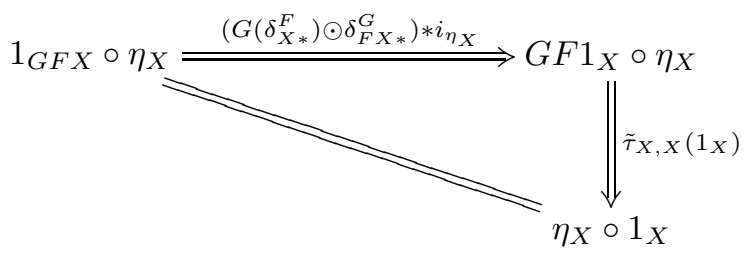

Hence $\left(\tilde{\tau}_{X, X}\left(1_{X}\right)\right)^{-1}=\left(G\left(\delta_{X *}^{F}\right) \odot \delta_{F X *}^{G}\right) * i_{\eta_{X}}$ as 2-cells. Note also that $\delta_{X *}^{G F}=$ $\left(G\left(\delta_{X *}^{F}\right) \odot \delta_{F X *}^{G}\right)$ by the definition of composition of pseudo functors. Using this, we see that diagram (23) becomes the outermost rectangle of the following diagram.

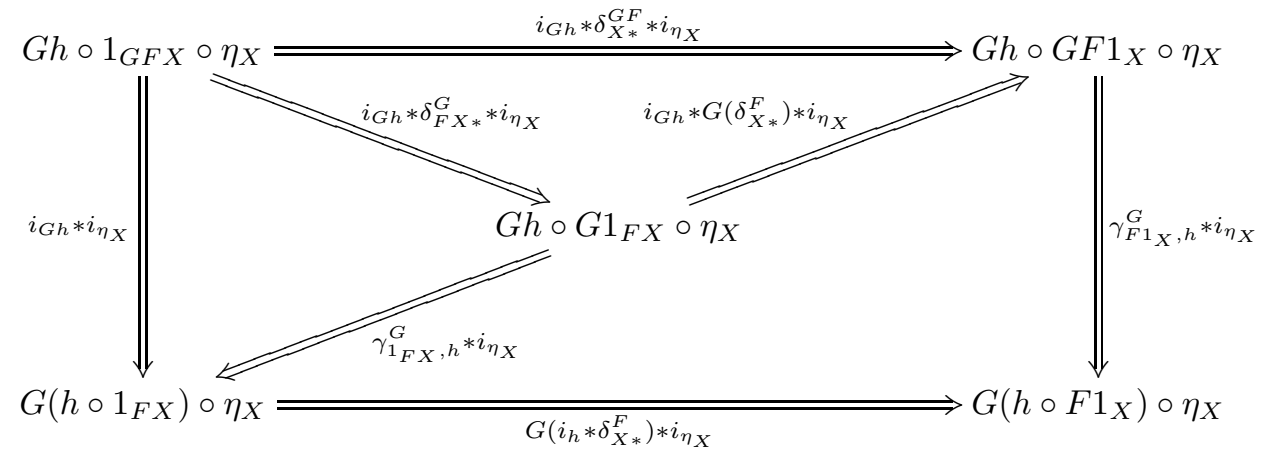

The upper left vertex of this diagram is the upper right vertex of diagram (23) and the composition of the top arrow and right vertical arrow of this diagram is the right vertical arrow of diagram (23). The top triangle of this diagram commutes by definition. The left triangle commutes by the unit axiom of the pseudo functor $G$ applied to the morphism $h: F X \rightarrow A$ of $\mathcal{A}$. The right quadrilateral commutes by the naturality of $\gamma_{-, h}^{G}$ and because $G\left(i_{h} * \delta_{X *}^{F}\right)=i_{G h} * G\left(\delta_{X *}^{F}\right)$. The morphism $\eta_{X}$ and the 2 -cell $i_{\eta_{X}}$ just tag along. Hence the outermost rectangle commutes and diagram (23) commutes. This implies that diagram (22) commutes. We conclude that $\tau^{\prime}$ satisfies the unit axiom required for $\phi_{-, A}$ to be a pseudo natural transformation.

I claim that $\tau^{\prime}$ satisfies the composition axiom required for $\phi_{-, A}$ to be a pseudo natural transformation. We must prove that for all morphisms $X \stackrel{f}{\longrightarrow} Y \stackrel{g}{\longrightarrow} Z$ of $\mathcal{X}$, i.e. for all morphisms $Z \stackrel{g^{o p}}{\longrightarrow} Y \stackrel{f^{o p}}{\longrightarrow} X$ of $\mathcal{X}^{o p}$, the diagram of 2-cells in Cat

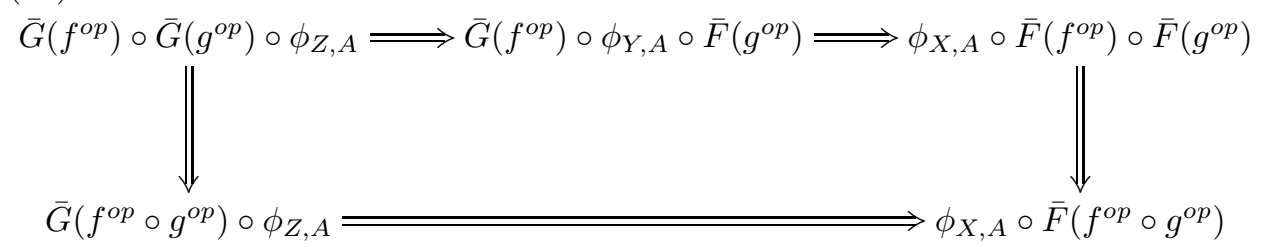


THOMAS M. FIORE

commutes. More precisely the diagram of 2-cells in Cat $(25)$

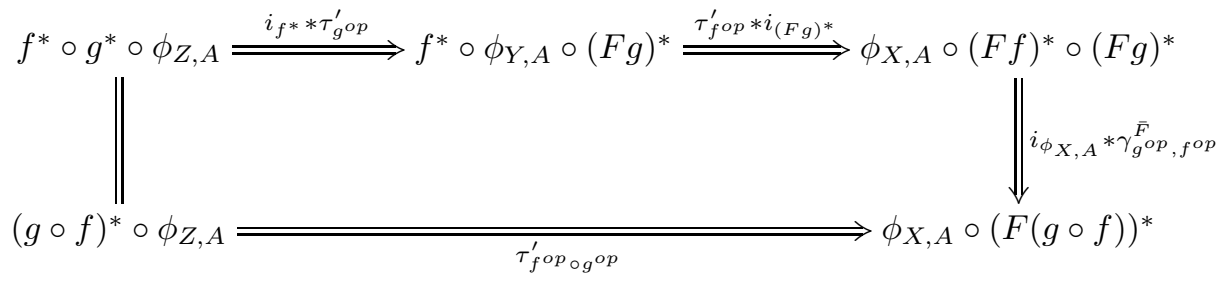

must commute. I evaluate this diagram on a morphism $h: F Z \rightarrow A$ of $\mathcal{A}$, fill in the diagram with more vertices, and cut the result down the middle column to get the left respectively right half below.

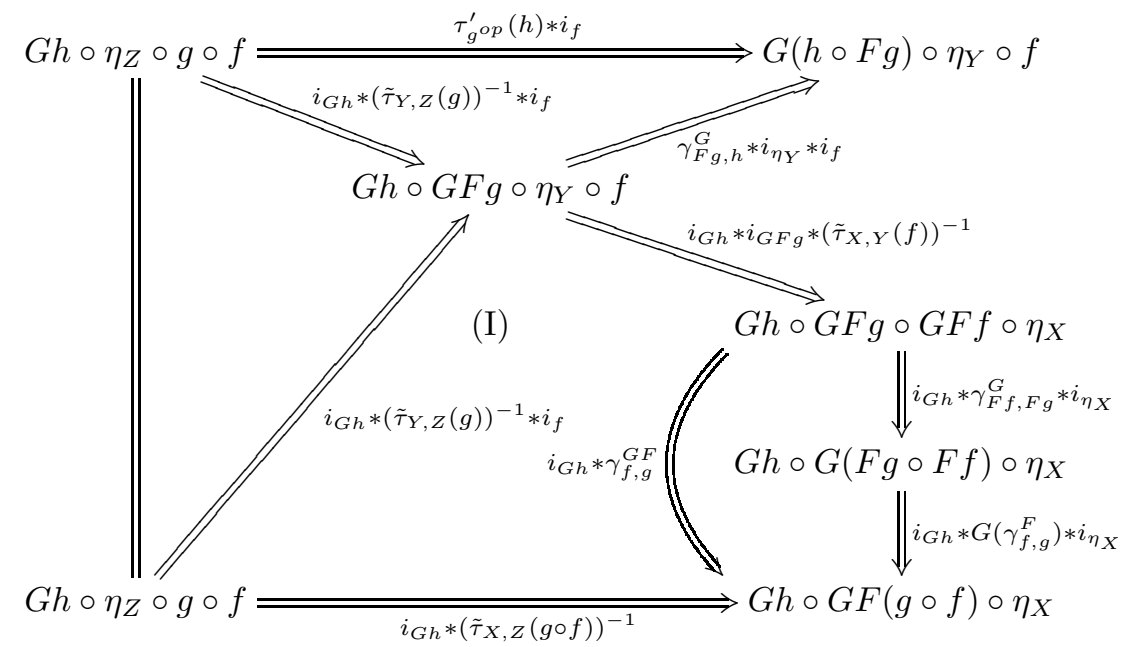

$(27)$

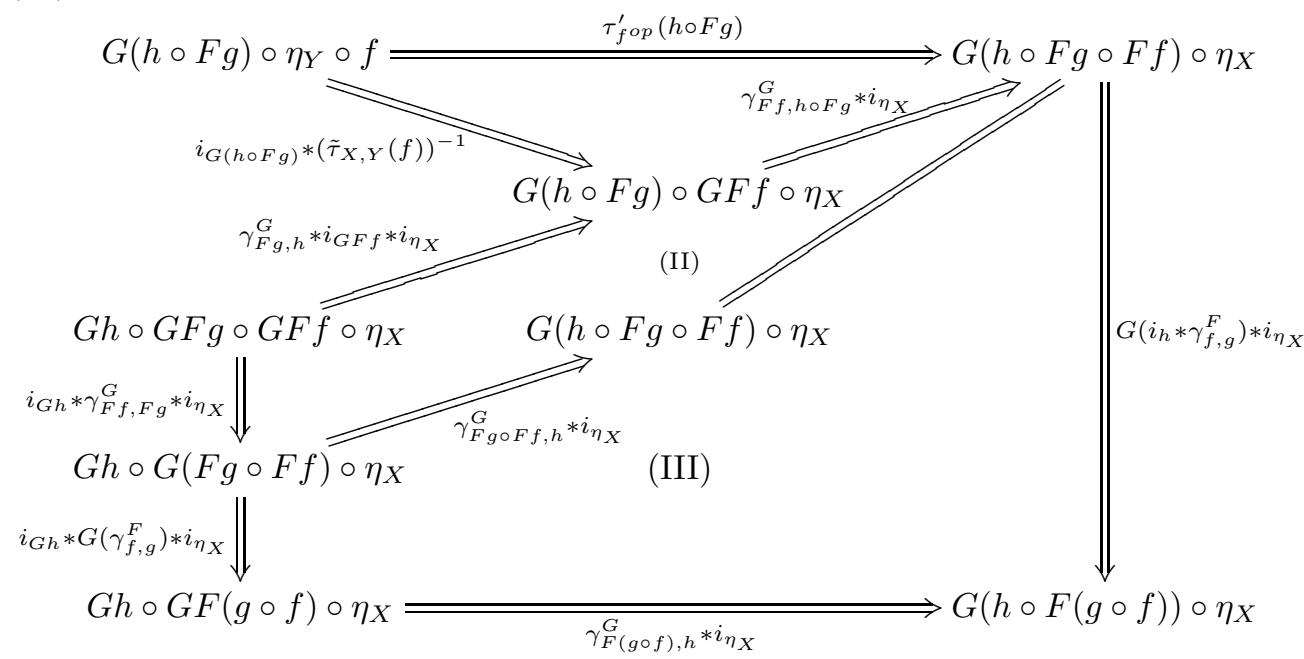


These are diagrams of 2-cells in $\mathcal{X}$. Subdiagram (I) commutes by the composition axiom applied to the morphisms $X \stackrel{f}{\longrightarrow} Y \stackrel{g}{\longrightarrow} Z$ for the pseudo natural transformation $\eta: 1_{\mathcal{X}} \Rightarrow G F$ with its coherence 2-cells $\tilde{\tau}$. Subdiagram (II) commutes by the composition axiom applied to the morphisms $F f, F g, h$ for the pseudo functor $G$ with its coherence 2-cells $\gamma^{G}$. The fifth arrow which is an equality symbol was only drawn for convenience. Subdiagram (III) commutes by the naturality of $\gamma^{G}$. All other subdiagrams commute by definition or by the interchange law. Therefore the outermost rectangle commutes when we put the two halves together. This outermost rectangle is diagram (25) evaluated on the morphism $h: F Z \rightarrow A$ of $\mathcal{A}$. Hence (25) and (24) commute. We conclude that $\tau^{\prime}$ satisfies the composition axiom required for $\phi_{-, A}$ to be a pseudo natural transformation.

Since $\phi_{-, A}$ with coherence 2-cells $\tau^{\prime}$ satisfies the unit axiom and composition axiom for pseudo natural transformations we conclude that $\phi_{-, A}$ is a pseudo natural transformation for fixed $A \in O b j \mathcal{A}$.

Lemma 8.11. Let $\mathcal{X}$ and $\mathcal{A}$ be 2-categories. Let $\mathcal{X} \underset{G}{\stackrel{F}{\rightleftarrows}} \mathcal{A}$ be pseudo functors between 2-categories. Let $\eta: 1_{\mathcal{X}} \Rightarrow G F$ be a pseudo natural transformation such that each $\eta_{X}: X \rightarrow G(F X)$ is a bi-universal arrow from $X$ to $G$. Let $\phi_{X, A}$ be defined as in Lemma [8.9 above. Then for fixed $X \in O b j \mathcal{X}$ the assignment Obj $\mathcal{A} \ni A \mapsto \phi_{X, A}$ denoted $\phi_{X,-}$ is pseudo natural.

Proof: $\quad$ Let $X$ be a fixed object of the 2-category $\mathcal{X}$ throughout the proof. I introduce new pseudo functors $\bar{F}$ and $\bar{G}$ different from those in the previous proof. Let $\bar{F}: \mathcal{A} \rightarrow C a t$ be the strict 2-functor obtained by fixing $X$ in the top row in the definition of bi-adjunction. This means $\bar{F}(A)=\operatorname{Mor}_{\mathcal{A}}(F X, A), \bar{F}(f)=f_{*}$, and for $\alpha: f \Rightarrow f^{\prime}$ we have $\bar{F}(\alpha)$ is the natural transformation $h \mapsto \alpha * i_{h}$. The 2 -functor $\bar{F}$ is strict because it is the composition of two strict 2-functors. Similarly let $\bar{G}: \mathcal{A} \rightarrow C a t$ be the pseudo functor obtained by fixing $X$ in the bottom row of the definition of bi-adjunction. This means $\bar{G}(A)=\operatorname{Mor}_{\mathcal{X}}(X, G A), \bar{G}(f)=(G f)_{*}$, and for $\alpha: f \Rightarrow f^{\prime}$ we have $\bar{G}(\alpha)$ is the natural transformation $h \mapsto G(\alpha) * i_{h}$. The pseudo functor $\bar{G}$ is pseudo because it is the composition of a pseudo functor and a strict functor. The definition of composition of pseudo functors then says that the coherence 2-cells for $\bar{G}$ are $\gamma_{f, g}^{\bar{G}}: h \mapsto \gamma_{f, g}^{G} * i_{h}$ for morphisms $f, g$ of $\mathcal{A}$ such that $g \circ f$ exists and $\delta_{A *}^{\bar{G}}: h \mapsto \delta_{A *}^{G} * i_{h}$ for $A \in O b j \mathcal{A}$. These are natural transformations, i.e. 2-cells in Cat, such that $\gamma_{f, g}^{\bar{G}}: \bar{G}(g) \circ \bar{G}(f) \Rightarrow \bar{G}(g \circ f)$ and $\delta_{A *}^{\bar{G}}: 1_{\bar{G}(A)} \Rightarrow \bar{G}\left(1_{A}\right)$. They are natural in $f$ and $g$ and they satisfy the required coherences for a pseudo functor.

I must show that $\phi_{X,-}$ is a pseudo natural transformation from $\bar{F}$ to $\bar{G}$. In other words I must display coherence 2-cells $\tau$ up to which $\phi_{X,-}$ is natural and satisfy the coherence diagrams involving $\gamma$ and $\delta$ from $\bar{F}$ and $\bar{G}$. For morphisms $k: A \rightarrow A^{\prime}$ 
of $\mathcal{A}$ define $\tau_{A, A^{\prime}}(k): e \mapsto \gamma_{e, k}^{G} * i_{\eta_{X}}$ to fill in the diagram

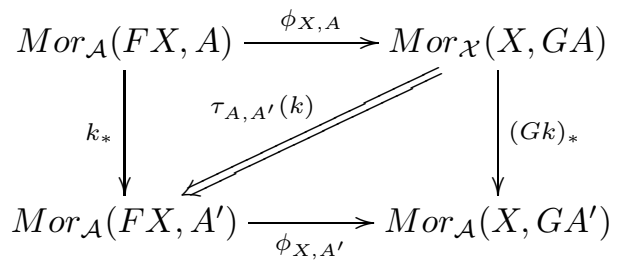

whose vertices are $\bar{F}(A), \bar{G}(A), \bar{G}\left(A^{\prime}\right)$, and $\bar{F}\left(A^{\prime}\right)$ read clockwise. The map $\tau_{A, A^{\prime}}(k)$ is a natural transformation (2-cell in $C a t$ ) between the indicated functors because $\gamma_{e, k}^{G}$ is natural in $e$. The assignment $\operatorname{Mor}_{\mathcal{A}}\left(A, A^{\prime}\right) \ni k \mapsto \tau_{A, A^{\prime}}(k)$ is a natural transformation $\left(\circ \phi_{X, A}\right) \circ \bar{G} \Rightarrow\left(\phi_{X, A^{\prime}} \circ\right) \circ \bar{F}$ because $\gamma_{e, k}^{G}$ is natural in $k$. Hence this family $\tau$ of natural transformations provides us with a candidate for the coherence 2-cells to make $\phi_{X,-}$ into a pseudo natural transformation.

I claim that $\tau$ satisfies the unit axiom for pseudo natural transformations. This entails proving that the diagram of 2-cells in Cat

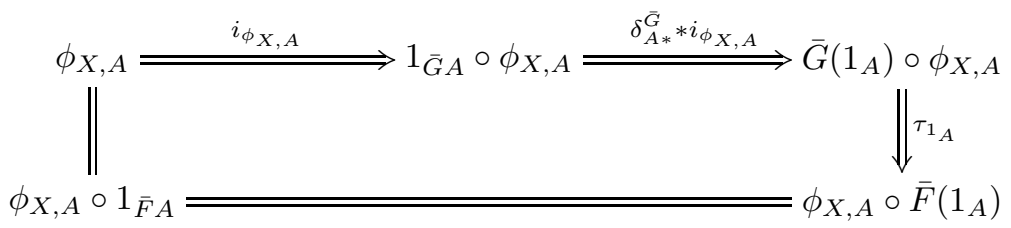

commutes for all $A \in O b j \mathcal{A}$. Evaluating this diagram on a morphism $e: F X \rightarrow A$ of $\mathcal{A}$ results in the diagram of 2 -cells

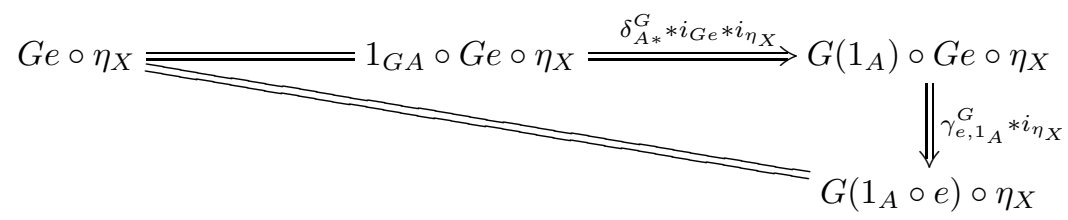

in $\mathcal{X}$ which commutes because of the unit axiom for the pseudo functor $G$. Hence $\tau$ satisfies the unit axiom for pseudo natural transformations.

I claim that $\tau$ satisfies the composition axiom for pseudo natural transformations.

This entails proving that for all morphisms $A \stackrel{f}{\longrightarrow} B \stackrel{g}{\longrightarrow} C$ in $\mathcal{A}$ the diagram of 2-cells in Cat

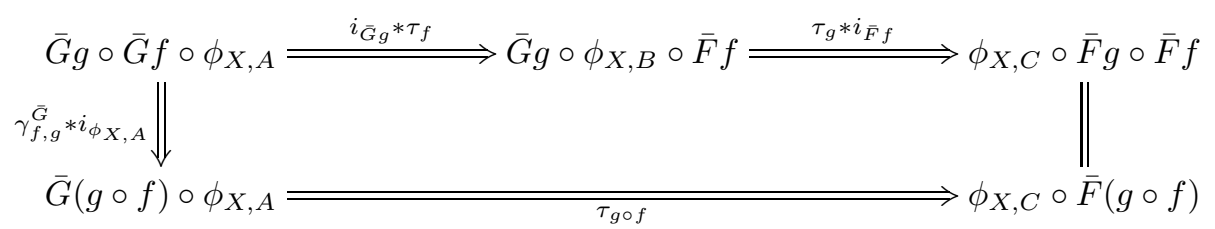


commutes. Evaluating this diagram on a morphism $e: F X \rightarrow A$ of $\mathcal{A}$ results in the diagram of 2-cells

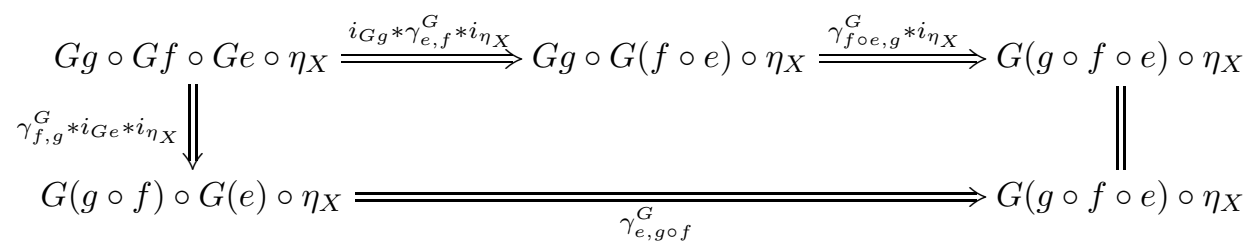

in $\mathcal{X}$, which commutes by the composition axiom for the pseudo functor $G$ applied to $F X \stackrel{e}{\longrightarrow} A \stackrel{f}{\longrightarrow} B \stackrel{g}{\longrightarrow} C$. Hence $\tau$ satisfies the composition axiom for pseudo natural transformations.

We conclude that $\phi_{X,-}$ is a pseudo natural transformation from $\bar{F}$ to $\bar{G}$ with coherence 2-cells defined by $\tau$.

Now we can finally state and prove the two main theorems of this section.

Theorem 8.12. Let $\mathcal{X}$ and $\mathcal{A}$ be 2-categories. Let $\mathcal{X} \underset{G}{\stackrel{F}{\rightleftarrows}} \mathcal{A}$ be pseudo functors. Then $F$ is a left bi-adjoint for $G$ if and only if there exists a pseudo natural transformation $\eta: 1_{\mathcal{X}} \Rightarrow G F$ such that $\eta_{X}: X \rightarrow G(F X)$ is a bi-universal arrow for all $X \in O b j \mathcal{X}$.

Proof: This follows immediately from the previous lemmas.

Theorem 8.13. Let $\mathcal{X}$ and $\mathcal{A}$ be 2-categories. Let $\mathcal{X} \longleftarrow{ }^{G} \mathcal{A}$ be a pseudo functor. Then there exists a left bi-adjoint for $G$ if and only if for every object $X \in O b j \mathcal{X}$ there exists an object $R \in O b j \mathcal{A}$ and a bi-universal arrow $\eta_{X}: X \rightarrow G(R)$ from $X$ to $G$.

Proof: By Lemma 8.7 the existence of a left bi-adjoint implies the existence of such a bi-universal arrow. Now we prove the other direction. Suppose we have such a bi-universal arrow for each $X \in \operatorname{Obj} \mathcal{X}$. Define $F X:=R$. The object $R \in \operatorname{Obj} \mathcal{A}$

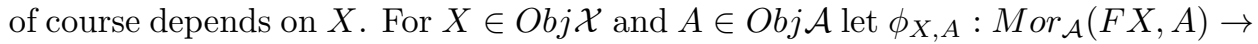
$\operatorname{Mor}_{\mathcal{X}}(X, G A)$ denote the functor $f^{\prime} \mapsto G f^{\prime} \circ \eta_{X}$ and $\gamma \mapsto G \gamma * i_{\eta_{X}}$. Let $\psi_{X, A}$ : $\operatorname{Mor}_{\mathcal{X}}(X, G A) \rightarrow \operatorname{Mor}_{\mathcal{A}}(F X, A)$ denote a right adjoint equivalence, which exists because $\eta_{X}$ is a bi-universal arrow. Let $\mu_{X, A}: \phi_{X, A} \circ \psi_{X, A} \Rightarrow 1_{M o r_{\mathcal{X}}(X, G A)}$ denote a counit for these adjoint functors. All of this implies that for any morphism $f: X \rightarrow G A$ there exists a morphism $f^{\prime}:=\psi_{X, A}(f)$ and a 2 -cell $\mu_{X, A}(f)$ as in the diagram.
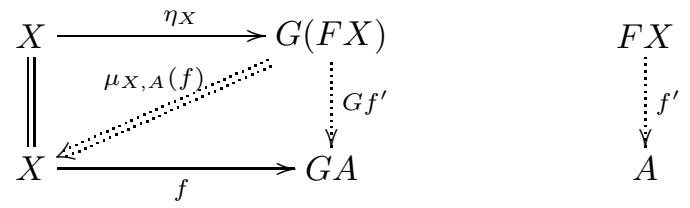

Moreover, this 2-cell $\mu_{X, A}(f)$ is a universal arrow from the functor $\phi_{X, A} \circ \psi_{X, A}$ to the object $f$ because all of the arrows of the counit of an adjunction are universal. This means that for any other morphism $\bar{f}^{\prime}: F X \rightarrow A$ and 2-cell $\nu$ as in the 
diagram
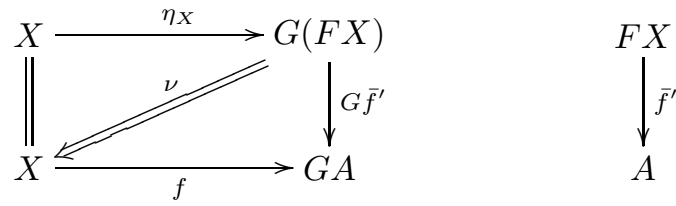

there exists a unique 2-cell $\nu^{\prime}: \bar{f}^{\prime} \Rightarrow f^{\prime}$ such that the following diagram commutes.
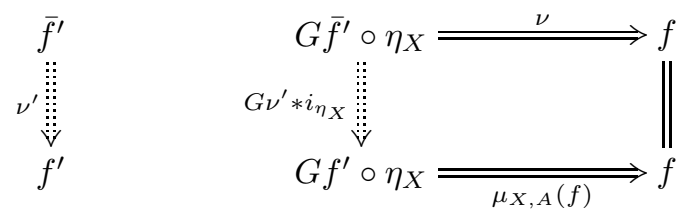

If $\nu$ is iso, this 2-cell $\nu^{\prime}: \bar{f}^{\prime} \Rightarrow f^{\prime}$ is also iso by the comments after Definition 8.2 The uniqueness and iso property of $\nu^{\prime}$ will be integral to defining the coherence isomorphisms and proving the coherence diagrams below.

After setting up this notation, I define a left bi-adjoint candidate $F$ for $G$. We already have $F$ defined for objects $X \in O b j \mathcal{X}$ above. For any morphism $h: X \rightarrow Y$ in $\mathcal{X}$ define $F h:=\psi_{X, F Y}\left(\eta_{Y} \circ h\right)$. For morphisms $h, h^{\prime}: X \rightarrow Y$ and any 2-cell $\gamma: h \Rightarrow h^{\prime}$ in $\mathcal{X}$ define $F \gamma:=\psi_{X, F Y}\left(i_{\eta_{Y}} * \gamma\right)$. Then the assignment is obviously a functor on any fixed hom set because of the interchange law and because $\psi_{X, F Y}$ preserves identity 2-cells and compositions of 2-cells. To define the coherence 2-cells $\delta_{X}^{F}$ I now use the uniqueness described above. Note that $F 1_{X}=\psi_{X, F X}\left(\eta_{X} \circ 1_{X}\right)$ satisfies the diagram
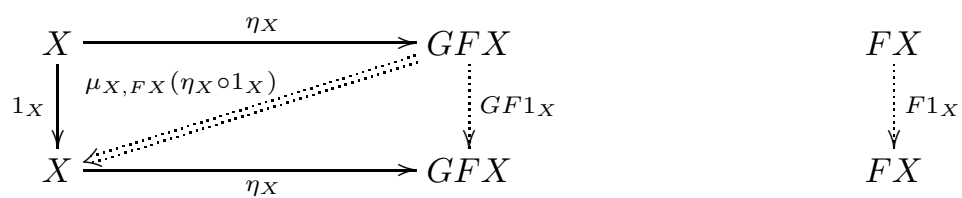

where $\mu_{X, F X}\left(\eta_{X} \circ 1_{X}\right)$ is universal. The arrow $1_{F X}$ satisfies
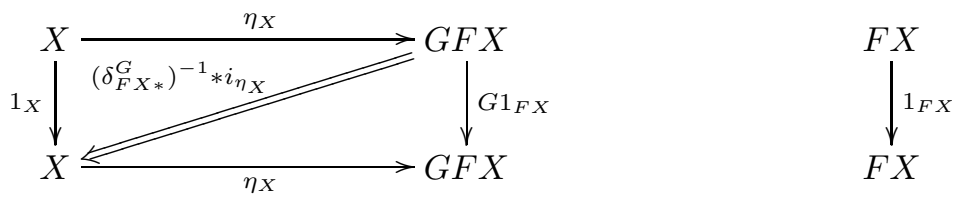

since $G$ is a pseudo functor. Let $\delta_{X *}^{F}: 1_{F X} \Rightarrow F 1_{X}$ be the unique 2-cell whose $\phi_{X, F X}$ image factors $\left(\delta_{F X *}^{G}\right)^{-1} * i_{\eta_{X}}$.

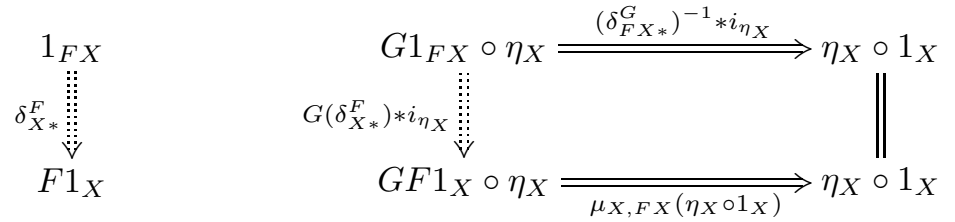

It exists by the universality of $\mu_{X, F X}\left(\eta_{X} \circ 1_{X}\right)$. The 2-cell $\delta_{X *}^{F}: 1_{F X} \Rightarrow F 1_{X}$ is iso because $\left(\delta_{F X_{*}}^{G}\right)^{-1} * i_{\eta_{X}}$ is iso. To define $\gamma_{f, g}^{F}$ for $X \stackrel{f}{\longrightarrow} Y \stackrel{g}{\longrightarrow} Z$ in $\mathcal{X}$ I similarly 
use the uniqueness. Note that $F(g \circ f)=\psi_{X, F Z}\left(\eta_{Z} \circ g \circ f\right)$ satisfies the diagram
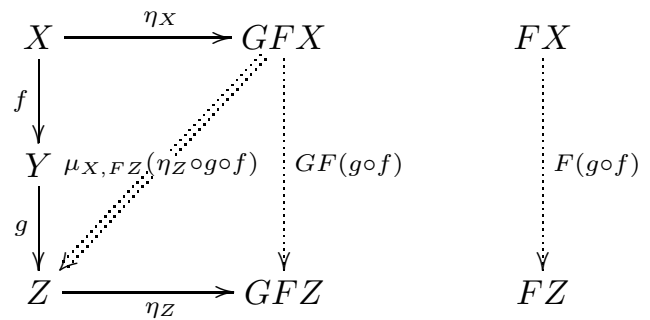

where the 2-cell $\mu_{X, F Z}\left(\eta_{Z} \circ g \circ f\right)$ is universal. The arrow $F g \circ F f$ satisfies
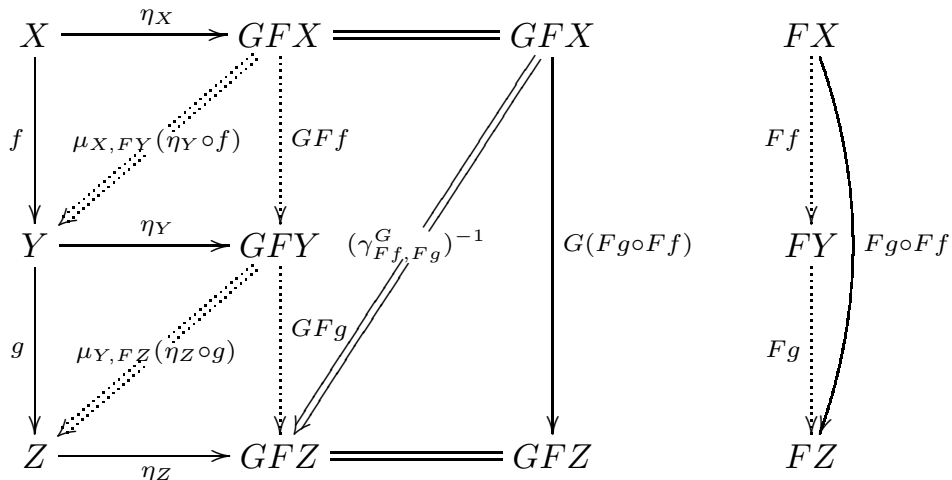

since $G$ is a pseudo functor. Let $\gamma_{f, g}^{F}: F g \circ F f \Rightarrow F(g \circ f)$ be the unique 2-cell whose $\phi_{X, F Z}$ image factors the composition of the 2-cells in (29) as follows.

$$
\begin{aligned}
& F g \circ F f \\
& \gamma_{f, g}^{F} \\
& F(g \circ f)
\end{aligned}
$$

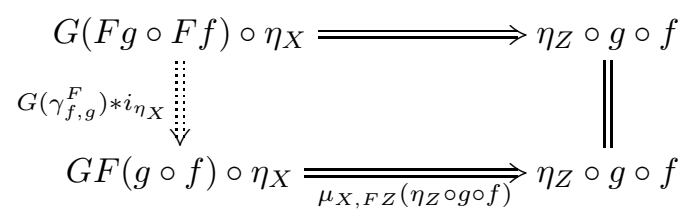

The top horizontal 2-cell in the previous diagram is the composition of the 2-cells in (29). The 2-cell $\gamma_{f, g}^{F}: F g \circ F f \Rightarrow F(g \circ f)$ is iso because the composition of 2 -cells in (29) is iso. Thus I have completely defined a left bi-adjoint candidate $F$ for $G$. Now I must show that the 2-cells do what they should in order for $F$ to be a pseudo functor.

I claim that $\gamma^{F}$ is natural in its two variables. I must show for morphisms $X \stackrel{f_{i}}{\longrightarrow} Y \stackrel{g_{i}}{\longrightarrow} Z$ in $\mathcal{X}$ and 2-cells $\alpha: f_{1} \Rightarrow f_{2}$ and $\beta: g_{1} \Rightarrow g_{2}$ in in $\mathcal{X}$ that

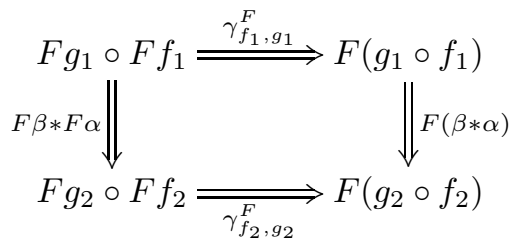

commutes. 
Toward this end, consider diagrams (32) and (33).

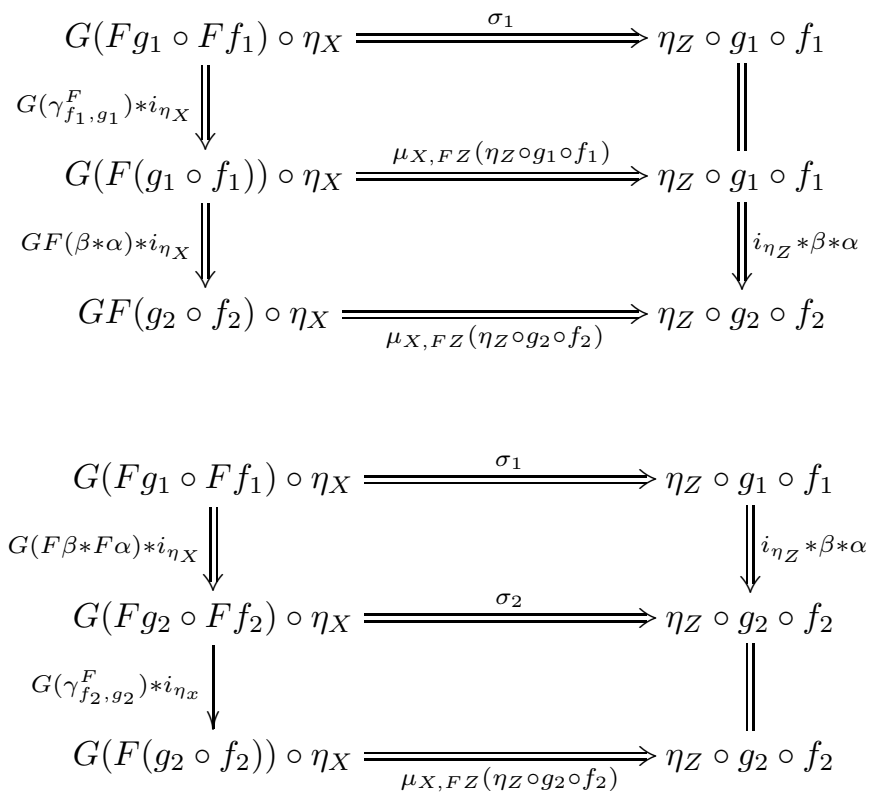

The top horizontal 2-cell $\sigma_{1}$ in both diagrams is the composition of the 2-cells in diagram (29) with $f, g$ replaced by $f_{1}, g_{1}$ respectively. The bottom horizontal 2-cell in each diagram is $\mu_{X, F Z}\left(\eta_{Z} \circ g_{2} \circ f_{2}\right)$. The center horizontal 2-cell $\sigma_{2}$ in (33) is the composition of the 2-cells in (29) with $f, g$ replaced by $f_{2}, g_{2}$ respectively. The top rectangle in (32) commutes because it is the analogue of (30) for $f_{1}, g_{1}$. The bottom rectangle in (32) commutes because of the naturality of $\mu_{X, F Z}: \phi_{X, F Z} \circ \psi_{X, F Z} \Rightarrow$

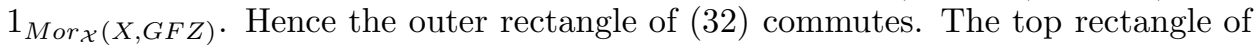
(33) commutes because of the naturality of $\left(\gamma^{G}\right)^{-1}, \mu_{X, F Y}$, and $\mu_{Y, F Z}$ by comparing with the 2-cells of (29). The bottom rectangle of (33) commutes because it is the analogue of (30) for $f_{2}, g_{2}$. Hence the outer rectangle of (33) commutes. From (32) and (33) we conclude that both $F(\beta * \alpha) \odot \gamma_{f_{1}, g_{1}}^{F}$ and $\gamma_{f_{2}, g_{2}}^{F} \odot(F \beta * F \alpha)$ have $\phi_{X, F Z}$ images which fill in the right diagram of (34).
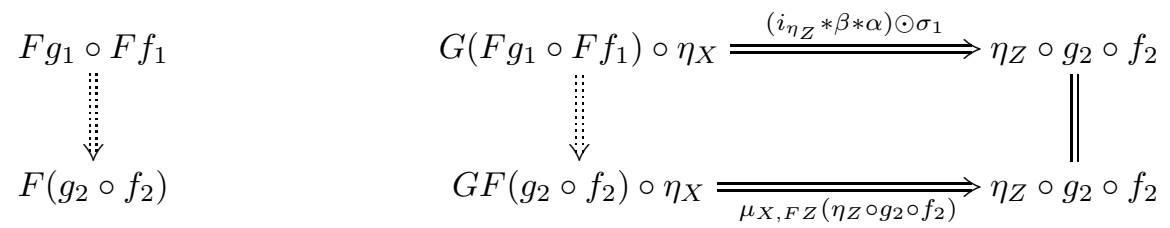

Since $\mu_{X, F Z}\left(\eta_{Z} \circ g_{2} \circ f_{2}\right)$ is universal, we conclude that $F(\beta * \alpha) \odot \gamma_{f_{1}, g_{1}}^{F}=\gamma_{f_{2}, g_{2}}^{F} \odot(F \beta * F \alpha)$ and thus $\gamma^{F}$ is natural in its two variables.

I claim that $\delta^{F}$ and $\gamma^{F}$ satisfy the unit axiom for pseudo functors. Let $X \in \operatorname{Obj\mathcal {X}}$ and let $f: X \rightarrow Y$ be a morphism of $\mathcal{X}$. I must show that $\gamma_{1_{X}, f}^{F}=\left(i_{F f} * \delta_{X *}^{F}\right)^{-1}$. By definition, $\gamma_{1_{X}, f}^{F}$ is the unique 2-cell $F f \circ F 1_{X} \Rightarrow F\left(f \circ 1_{X}\right)$ such that the 
composition of 2-cells

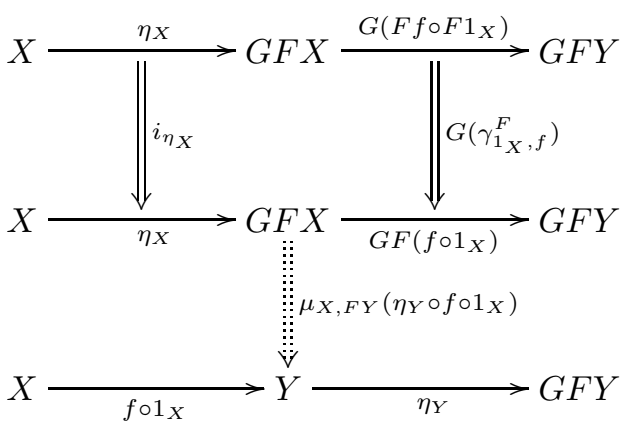

is the same as the composition of 2-cells

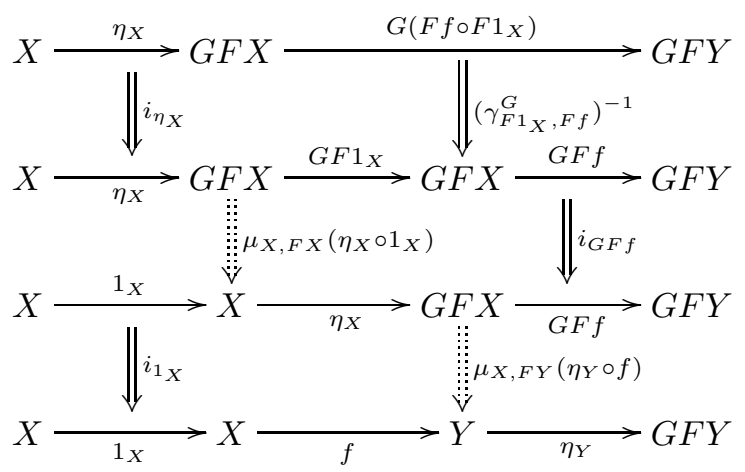

where universal 2-cells are drawn with dotted double arrows for clarity. I show that $\left(i_{F f} * \delta_{X *}^{F}\right)^{-1}$ is a 2-cell with this defining property for $\gamma_{1_{X}, f}^{F}$.

Since $\gamma^{G}$ is natural we can rewrite the first horizontal 2-cell composition in (36) as the composition of the first three 2-cells in the equal diagram (37).

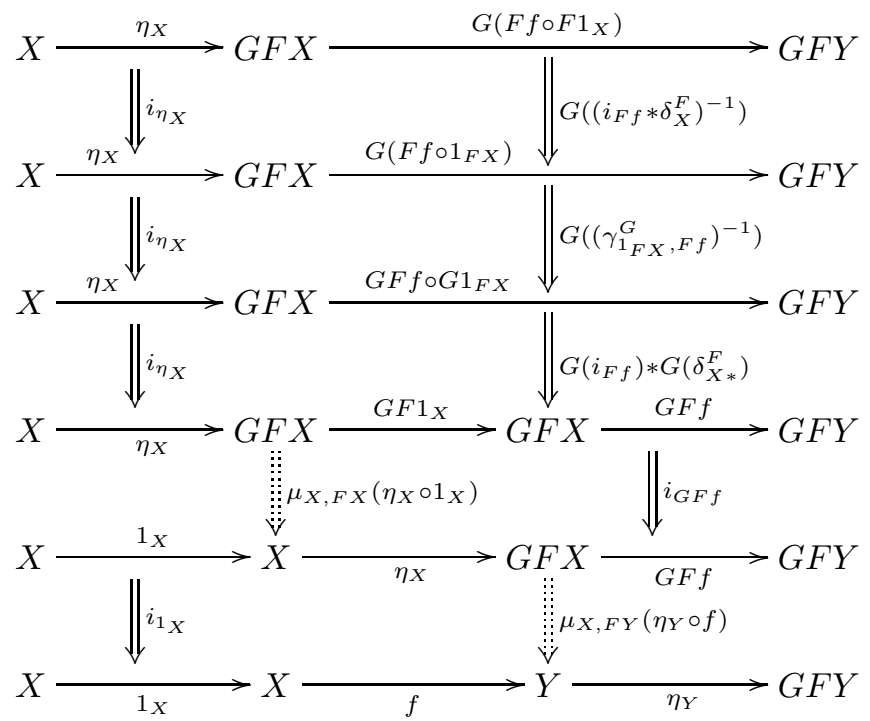


By the unit axiom for $G$, the definition of $\delta_{X}^{F}$, and the interchange law we see that the second horizontal composition in (37) is

$\left(\gamma_{1_{F X}, F f}^{G}\right)^{-1} * i_{\eta_{X}}=i_{G F f} * \delta_{F X *}^{G} * i_{\eta_{X}}$

$=i_{G F f} *\left(\mu_{X, F X}\left(\eta_{X} \circ 1_{X}\right) \odot\left(G\left(\delta_{X *}^{F}\right) * i_{\eta_{X}}\right)\right)^{-1}$

$=\left(G\left(i_{F f}\right) * G\left(\delta_{X *}^{F}\right)^{-1} * i_{\eta_{X}}\right) \odot\left(i_{G F f} * \mu_{X, F X}\left(\eta_{X} \circ 1_{X}\right)\right)^{-1}$

Substituting this in (37) for $\left(\gamma_{1_{F X}, F f}^{G}\right)^{-1} * i_{\eta_{X}}$ we see that the second horizontal composition in (37) cancels with the third and the fourth, leaving only

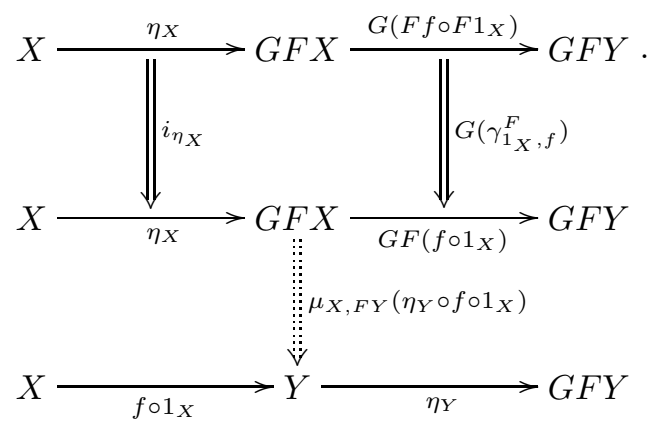

We see that the 2-cell compositions of (35), (36), (37), and (38) are all equal. Hence the 2-cell compositions (35) and (38) are equal and by universality of the 2-cell $\mu_{X, F Y}\left(\eta_{Y} \circ f \circ 1_{X}\right)$ we have $\gamma_{1_{X}, f}^{F}=\left(i_{F f} * \delta_{X *}^{F}\right)^{-1}$. The other half of the unit axiom can be verified similarly. We conclude that $\delta^{F}$ and $\gamma^{F}$ satisfy the unit axiom for pseudo functors.

I claim that $\gamma^{F}$ satisfies the composition axiom for pseudo functors. Let $W \stackrel{f}{\longrightarrow} X \stackrel{g}{\longrightarrow} Y \stackrel{h}{\longrightarrow} Z$ be morphisms of $\mathcal{X}$. I must show that $\gamma_{f, h \circ g}^{F}=\gamma_{g \circ f, h}^{F} \odot\left(i_{F h} * \gamma_{f, g}^{F}\right) \odot\left(\gamma_{g, h}^{F} * i_{F f}\right)^{-1}$. By definition $\left(\gamma_{f, h \circ g}^{F}\right)^{-1}$ is the unique 2-cell $F(h \circ g \circ f) \Rightarrow F(h \circ g) \circ F f$ such that the composition of 2-cells

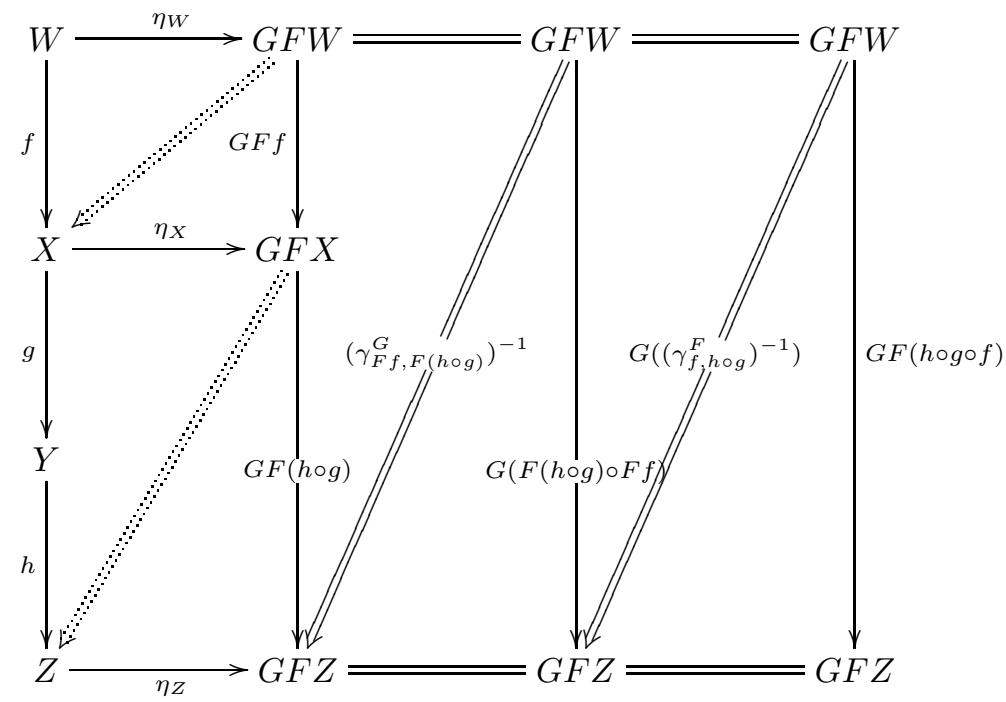

is the same as the universal 2-cell $\mu_{X, F Z}\left(\eta_{Z} \circ h \circ g \circ f\right)$. For clarity I continue to draw the universal 2-cells as dotted double arrows. I prove that replacing $\left(\gamma_{f, h \circ g}^{F}\right)^{-1}$ in (39) by $\left(\gamma_{g \circ f, h}^{F} \odot\left(i_{F h} * \gamma_{f, g}^{F}\right) \odot\left(\gamma_{g, h}^{F} * i_{F f}\right)^{-1}\right)^{-1}$ still gives $\mu_{X, F Z}\left(\eta_{Z} \circ h \circ g \circ f\right)$. 
After that we conclude

$\gamma_{f, h \circ g}^{F}=\gamma_{g \circ f, h}^{F} \odot\left(i_{F h} * \gamma_{f, g}^{F}\right) \odot\left(\gamma_{g, h}^{F} * i_{F f}\right)^{-1}$ by the universality of the 2-cell $\mu_{X, F Z}\left(\eta_{Z} \circ h \circ g \circ f\right)$. To this end, I claim that the composition

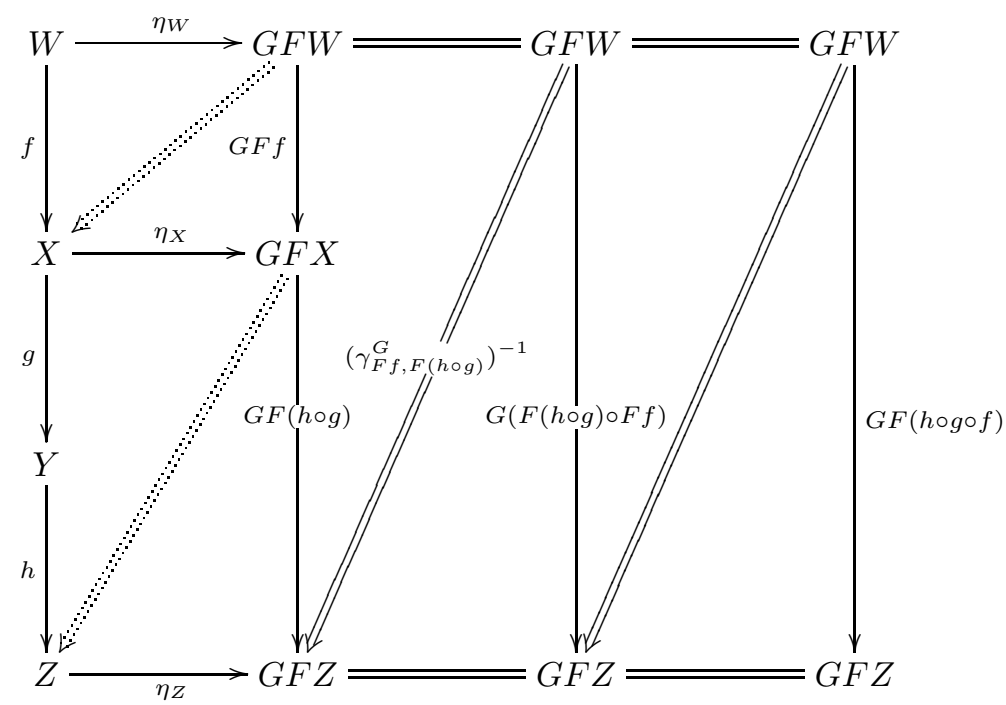

is the same as $\mu_{X, F Z}\left(\eta_{Z} \circ h \circ g \circ f\right)$, where the rightmost 2-cell is $G\left(\left(\gamma_{g \circ f, h}^{F} \odot\left(i_{F h} *\right.\right.\right.$ $\left.\left.\left.\gamma_{f, g}^{F}\right) \odot\left(\gamma_{g, h}^{F} * i_{F f}\right)^{-1}\right)^{-1}\right)$. I do this by transforming (40) to a diagram know to be $\mu_{X, F Z}\left(\eta_{Z} \circ h \circ g \circ f\right)$. The naturality of $\gamma^{G}$ guarantees that

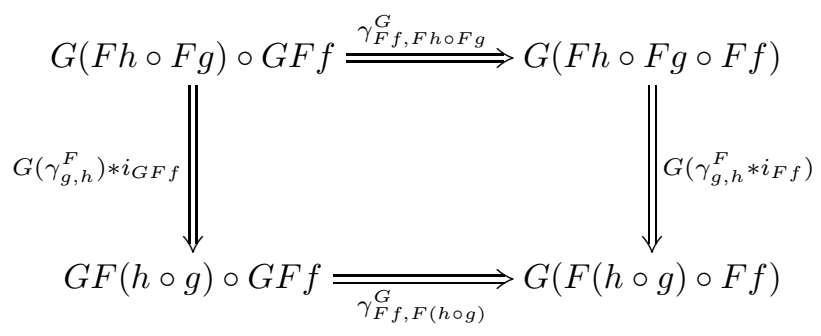


commutes. Using this commutivity to substitute for $\left(\gamma_{F f, F(h \circ g)}^{G}\right)^{-1}$ in (40) and cancelling $G\left(\gamma_{g, h}^{F} * i_{F f}\right)^{-1} \odot G\left(\gamma_{g, h}^{F} * i_{F f}\right)$ gives

(41)

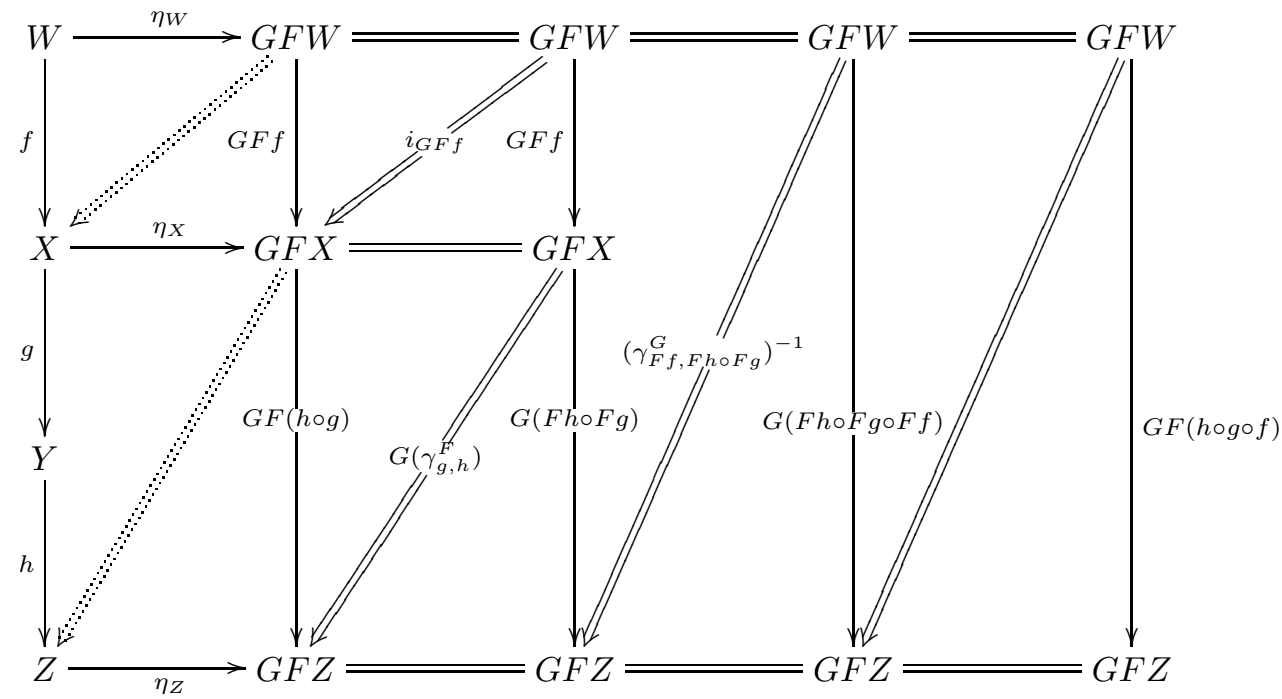

where the right 2-cell is $G\left(\left(i_{F h} * \gamma_{f, g}^{F}\right)^{-1} \odot\left(\gamma_{g \circ f, h}^{F}\right)^{-1}\right)$. We have also implicitly used the fact that $G$ preserves the vertical composition of 2-cells. By the definition of $\gamma_{g, h}^{F}$, the lower left two rectangles of (41) can be rewritten to give the equal composition (42).

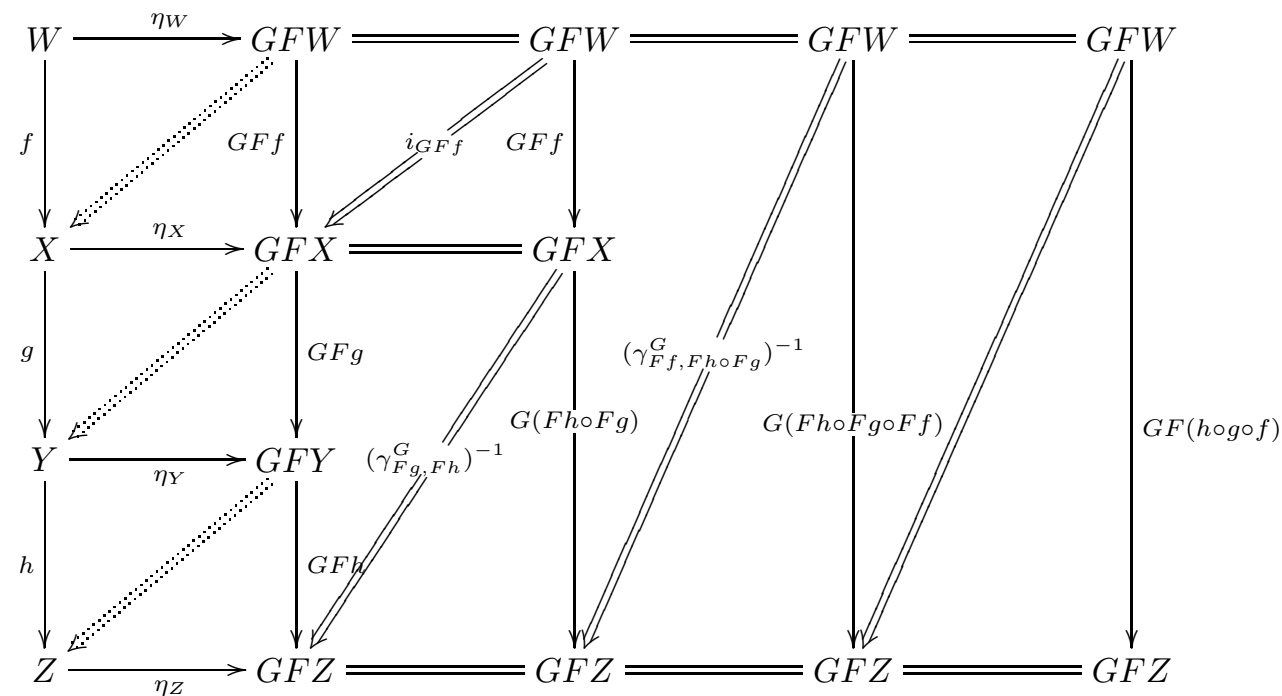


Recall that the composition axiom for the pseudo functor $G$ guarantees the commutivity of the following diagram.

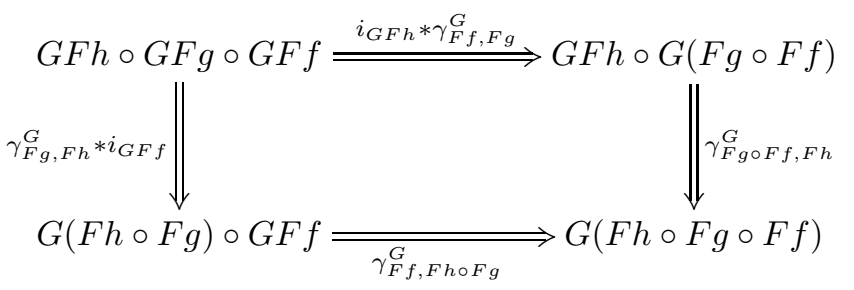

Using this composition axiom for the pseudo functor $G$ we can replace the middle two columns of 2-cells in (42) to get the equal composition (43).

(43)

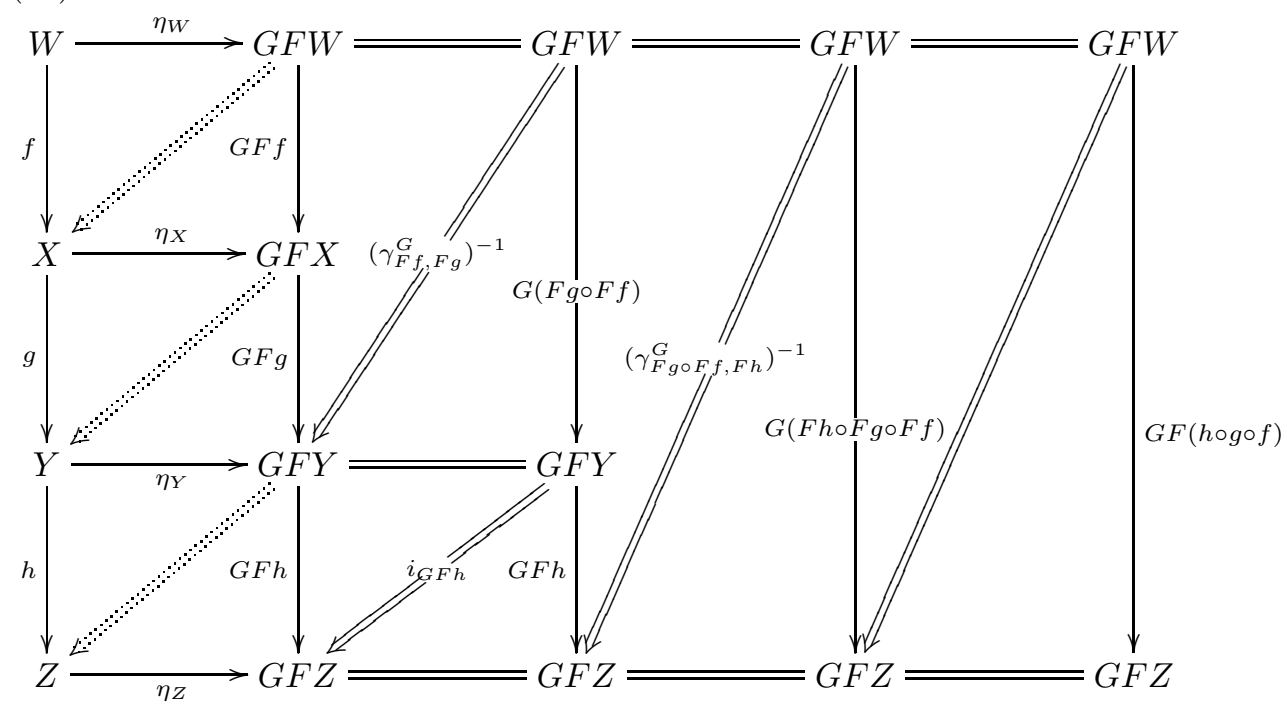

In (43) the right 2-cell is again $G\left(\left(i_{F h} * \gamma_{f, g}^{F}\right)^{-1} \odot\left(\gamma_{g \circ f, h}^{F}\right)^{-1}\right)$ as in (41) and (42). By the definition of $\gamma_{f, g}^{F}$ we can rewrite the upper left three rectangles of (43) to 
obtain (44), which has $G\left(\left(i_{F h} * \gamma_{f, g}^{F}\right)^{-1} \odot\left(\gamma_{g \circ f, h}^{F}\right)^{-1}\right)$ as its right 2-cell.

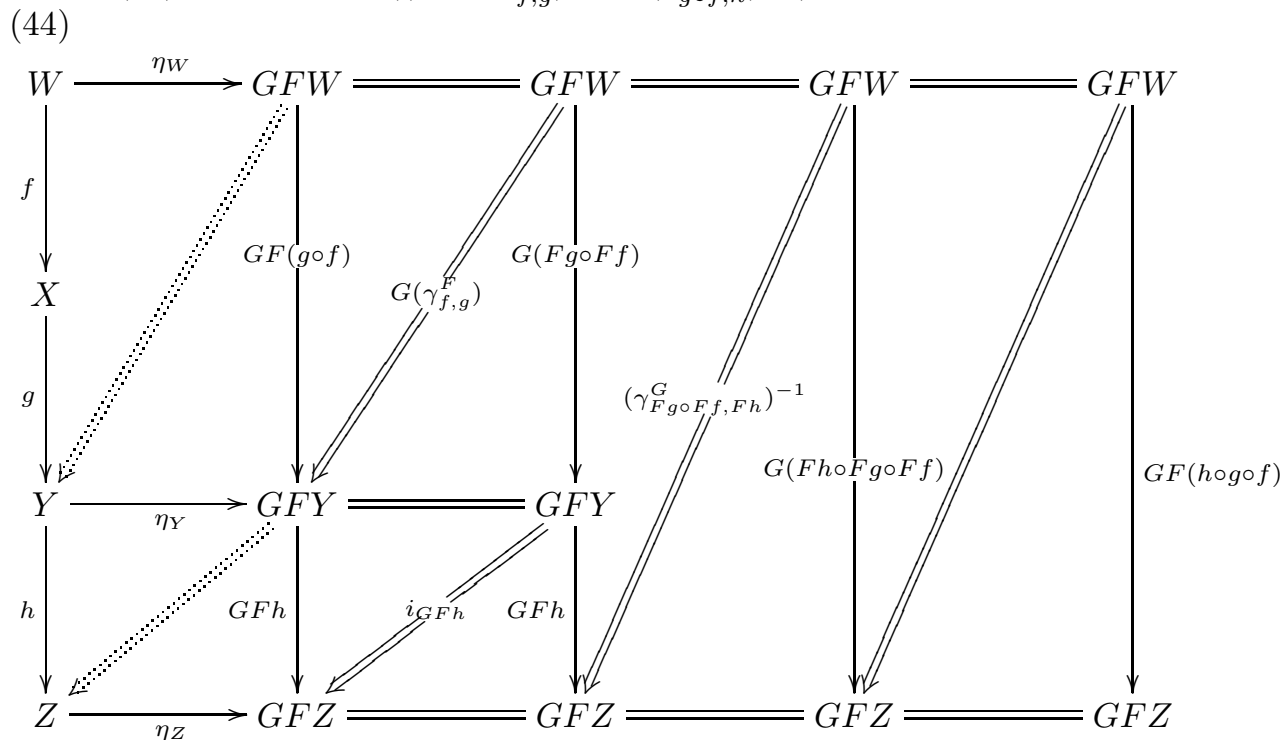

The naturality of $\gamma^{G}$ implies that the diagram

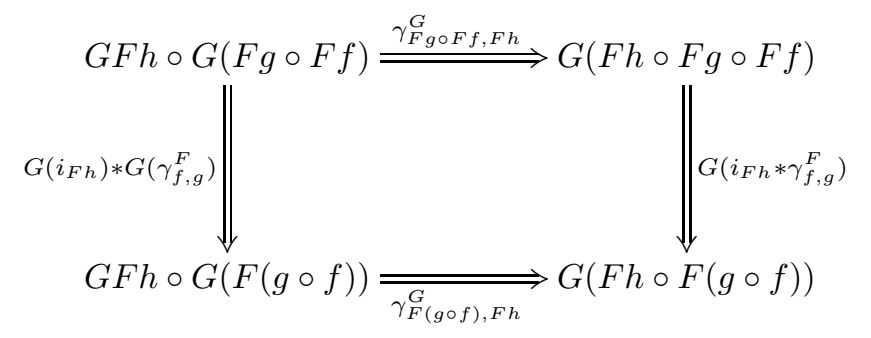

commutes. Using its commutivity, we can rewrite (44) by combining its middle two columns of 2-cells with $G\left(\left(i_{F h} * \gamma_{f, g}^{F}\right)^{-1}\right)$ from the last column to get 45.

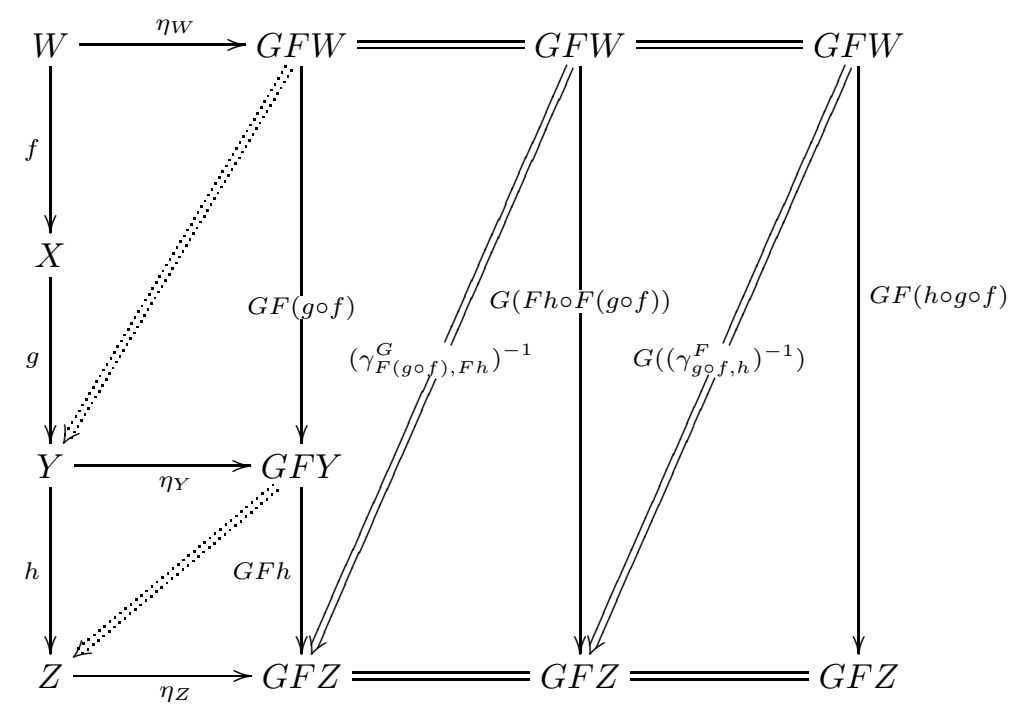


But by the definition of $\gamma_{g \circ f, h}^{F}$, the composition of 2-cells in (45) is precisely $\mu_{X, F Z}\left(\eta_{Z} \circ h \circ g \circ f\right)$. Since the compositions of 2-cells in the diagrams (40) through (45) are all equal, we conclude that the composition of 2-cells in (40) is $\mu_{X, F Z}\left(\eta_{Z} \circ h \circ g \circ f\right)$. We conclude that $\gamma_{f, h \circ g}^{F}=\gamma_{g \circ f, h}^{F} \odot\left(i_{F h} * \gamma_{f, g}^{F}\right) \odot\left(\gamma_{g, h}^{F} * i_{F f}\right)^{-1}$ by the universality of $\mu_{X, F Z}\left(\eta_{Z} \circ h \circ g \circ f\right)$. Therefore $\gamma^{F}$ satisfies the composition axiom for pseudo functors.

In summary, I have constructed a pseudo functor $F: \mathcal{X} \rightarrow \mathcal{A}$ with coherence 2cells $\delta^{F}$ and $\gamma^{F}$ and I have shown that they satisfy the unit axiom and composition axiom for pseudo functors.

Next I have to show that $F$ is a left bi-adjoint using the previous theorem. By

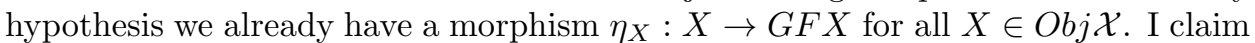
that the assignment $X \mapsto \eta_{X}$ is a pseudo natural transformation from $1_{\mathcal{X}}$ to $G F$. I need to define the 2-cells up to which $\eta$ is natural. For a morphism $f: X \rightarrow Y$ of $\mathcal{X}$ define $\tau_{f}:=\mu_{X, F Y}\left(\eta_{Y} \circ f\right)$. Then the diagram

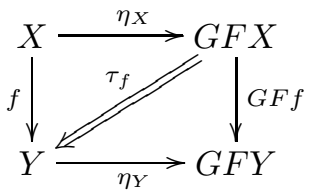

illustrates the source and target of the 2-cell. The map $f \mapsto \tau_{f}$ is natural because $\mu_{X, F Y}$ is a natural transformation. More precisely let $\alpha: f_{1} \Rightarrow f_{2}$ be a 2-cell in $\mathcal{X}$ and let $f_{1}, f_{2}: X \rightarrow Y$ be morphisms in $\mathcal{X}$. Then

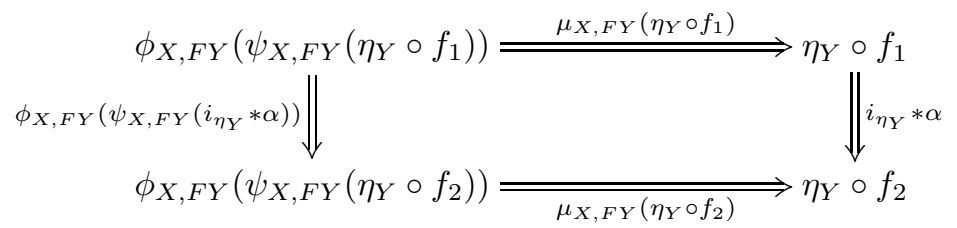

commutes by the naturality of $\mu_{X, F Y}$. By the definitions of $F, \tau_{f_{1}}$, and $\tau_{f_{2}}$ diagram (46) is the same as the diagram

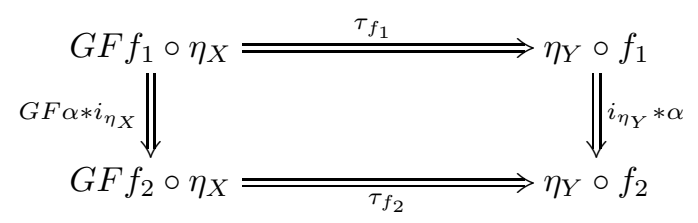

which says $f \mapsto \tau_{f}$ is natural. The map $f \mapsto \tau_{f}$ satisfies the unit axiom for pseudo natural transformations because of (28) and the definition of $\delta^{G F}$ for the composite pseudo functor $G F$. The map $f \mapsto \tau_{f}$ satisfies the composition axiom for pseudo natural transformations because of (30), (29), and the definition of $\gamma^{G F}$ for the composite pseudo functor $G F$. Hence $\eta: 1_{\mathcal{X}} \Rightarrow G F$ is a pseudo natural transformation with coherence 2-cells $\tau$.

By the previous theorem, the constructed pseudo functor $F$ is a left bi-adjoint because $\eta: 1_{\mathcal{X}} \Rightarrow G F$ is a pseudo natural transformation such that $\eta_{X}: X \rightarrow$ $G(F X)$ is a bi-universal arrow for all $X \in O b j \mathcal{X}$.

We can summarize the previous two theorems in way similar to Mac Lane's theorem on page 83 of [39] as follows. 
Theorem 8.14. A bi-adjunction $\langle F, G, \phi\rangle: \mathcal{X} \rightarrow \mathcal{A}$ can be described up to 2-cell by either of the following data:

(1) Pseudo functors

$$
\mathcal{X} \underset{G}{\stackrel{F}{\rightleftarrows}} \mathcal{A}
$$

and a pseudo natural transformation $\eta: 1_{\mathcal{X}} \Rightarrow G F$ such that each $\eta_{X}$ : $X \rightarrow G F X$ is a bi-universal arrow from $X$ to $G$. Then $\phi_{X, A}$ is defined by $\phi_{X, A}(f)=G f \circ \eta_{X}$.

(2) A pseudo functor $G: \mathcal{A} \rightarrow \mathcal{X}$, for each $X \in \operatorname{Obj\mathcal {X}}$ an object $R \in \mathcal{A}$ depending on $X$, and for each $X \in O \operatorname{Obj\mathcal {X}}$ a bi-universal arrow $\eta_{X}: X \rightarrow$ $G R$ from $X$ to $G$. Then the pseudo functor $F$ satisfies $F X=R$ on objects

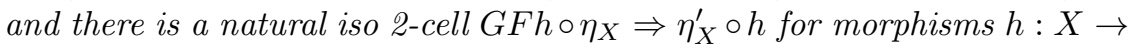
$X^{\prime}$.

Proof: Uniqueness will be proven below.

Similar things can be formulated for bicounits. From 1-category theory we know that any two left adjoints to a functor are naturally isomorphic. A similar statement can be made for left bi-adjoints, although one needs the concept of pseudo natural pseudo isomorphism.

Definition 8.5. Let $F, F^{\prime}: \mathcal{X} \rightarrow \mathcal{A}$ be pseudo functors. Then a pseudo natural transformation $\alpha: F \Rightarrow F^{\prime}$ is called a pseudo natural pseudo isomorphism or pseudo natural equivalence if there exists a pseudo natural transformation $\alpha^{\prime}: F^{\prime} \Rightarrow F$ and there exist iso modifications $\alpha \odot \alpha^{\prime} \rightsquigarrow 1_{F^{\prime}}$ and $\alpha^{\prime} \odot \alpha \rightsquigarrow 1_{F}$.

Theorem 8.15. Let $F, F^{\prime}: \mathcal{X} \rightarrow \mathcal{A}$ be left bi-adjoints for a pseudo functor $G:$ $\mathcal{A} \rightarrow \mathcal{X}$. Then there exists a pseudo natural pseudo isomorphism $\alpha: F \Rightarrow F^{\prime}$

Proof: $\quad$ For $X \in \operatorname{Obj} \mathcal{X}$, let $\eta_{X}: X \rightarrow G F X$ and $\eta_{X}^{\prime}: X \rightarrow G F^{\prime} X$ be the biuniversal arrows obtained from the bi-adjunctions as in the theorems above. Then by Lemma 8.4 there exists a pseudo isomorphism $\alpha_{X}: F X \rightarrow F^{\prime} X$ and a pseudo inverse $\alpha_{X}^{\prime}: F^{\prime} X \rightarrow F X$ as well as 2-cells $\alpha_{X}^{\prime} \circ \alpha_{X} \Rightarrow 1_{F X}$ and $\alpha_{X} \circ \alpha_{X}^{\prime} \Rightarrow 1_{F^{\prime} X}$. It can be shown that the assignments $X \rightarrow \alpha_{X}$ and $X \rightarrow \alpha_{X}^{\prime}$ are pseudo natural and the 2-cells determine modifications $\alpha^{\prime} \odot \alpha \rightsquigarrow 1_{F}$ and $\alpha \odot \alpha^{\prime} \rightsquigarrow 1_{F^{\prime}}$.

For example, I construct the coherence 2-cell $\tau^{\alpha}$ up to which $\alpha$ is natural. For $f \in \operatorname{Mor}_{\mathcal{X}}(X, Y)$ we have the following two diagrams.
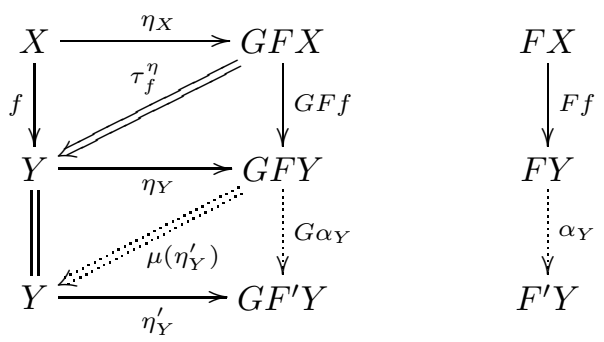

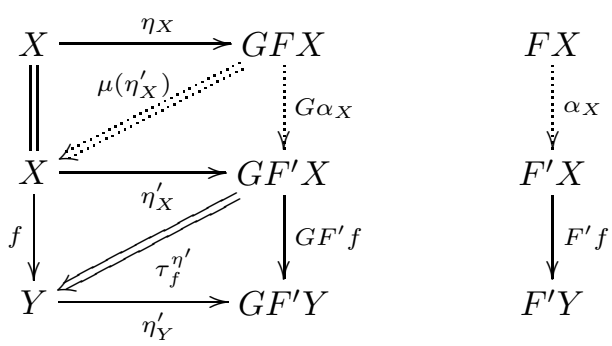

But they can also be filled in as

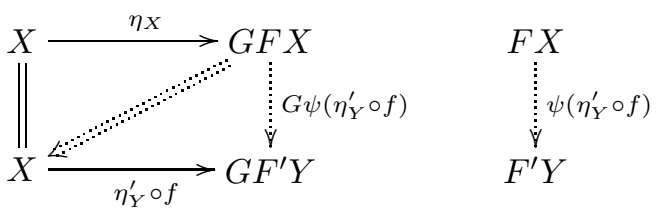

where the dashed 2-cell is universal. The universality gives us iso 2-cells $\nu_{f}$ and $\nu_{f}^{\prime}$ as in

$$
\left.F^{\prime} f \circ \alpha_{X} \Longleftarrow \nu_{f}^{\prime}=\eta_{Y}^{\prime} \circ f\right) \Longleftarrow \alpha_{Y} \circ F f
$$

whose $\phi$ images factor (via the universal 2-cell) the 2-cells in (48) and (49) precomposed with the appropriate $\left(\gamma^{G}\right)^{-1}$ 's. Define $\tau_{f}^{\alpha}:=\tau_{f}:=\left(\nu_{f}\right)^{-1} \odot \nu_{f}^{\prime}$. This is the coherence 2-cell up to which $\alpha$ will be natural.

A sketch of the naturality of $f \mapsto \tau_{f}$ goes as follows. Let $\beta: f_{1} \Rightarrow f_{2}$ be a 2-cell between $f_{1}, f_{2}: X \rightarrow Y$. Then we must show that the outer rectangle of

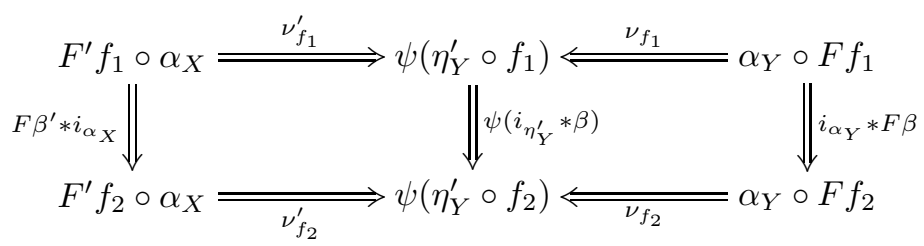

commutes. One does this by showing that the individual inner squares commute by applying $\phi$ and using the universality and the fact that $\mu$ is a natural isomorphism. It also involves the naturality of the $\gamma^{G}$ 's.

One can also show that $\tau$ satisfies the composition and unit axiom, although it is lengthy. Lastly one must verify that the 2 -cell assignments at the start actually give modifications $\alpha^{\prime} \odot \alpha \rightsquigarrow 1_{F}$ and $\alpha \odot \alpha^{\prime} \rightsquigarrow 1_{F^{\prime}}$.

Thus, any two left bi-adjoints are pseudo naturally pseudo isomorphic.

There is a relationship between bi(co)limits and bi-adjoints, just like for (co)limits and adjoints.

Remark 8.16. Let $\mathcal{C}$ be a 2-category with bicolimits and let $\mathcal{J}$ be a 1-category. Let $\mathcal{C}^{\mathcal{J}}$ be the category with objects pseudo functors $\mathcal{J} \rightarrow \mathcal{C}$, morphisms pseudo natural transformations, and 2-cells the modifications. This is a 2-category. Let $\Delta: \mathcal{C} \rightarrow \mathcal{C}^{\mathcal{J}}$ be the constant 2-functor. Then laxcolim $: \mathcal{C}^{\mathcal{J}} \rightarrow \mathcal{C}$ is a left bi-adjoint for $\Delta$ and the arrows of the bi-unit constructed in Theorem 8.12] are the universal 
pseudo cones. Similarly, laxlim: $\mathcal{C}^{\mathcal{J}} \rightarrow \mathcal{C}$ is a right bi-adjoint for $\Delta$ and the arrows of the bicounit are the universal pseudo cones.

\section{Forgetful 2-Functors For Pseudo Algebras}

Next I show that forgetful 2-functors for pseudo algebras have left bi-adjoints. Let us consider the strict case as an example of what we do below. Let $S$ be the theory of abelian groups and let $T$ by the theory of rings. Then we have an inclusion $S \hookrightarrow T$. Let $X$ be a discrete $T$-algebra, i.e. $X$ is a set and we have a morphism of theories $T \rightarrow \operatorname{End}(X)$. Then $X$ can be made into an $S$-algebra by the composite map of theories $S \hookrightarrow T \rightarrow \operatorname{End}(X)$. This precomposition with the inclusion arrow forgets the ring structure on the set $X$ and results in the underlying abelian group. This precomposition with the inclusion defines the forgetful functor from the category of rings to the category of abelian groups. It has a left adjoint which is the appropriate free functor. Similarly, for any morphism of theories $S \rightarrow T$ we have a forgetful 2-functor from pseudo $T$-algebras to pseudo $S$-algebras for which a left bi-adjoint exists. Blackwell, Kelly, and Power have shown that left bi-adjoints exist for the analogous 2-functor on 2-categories of strict algebras over a 2-monad with pseudo morphisms in [9]. Lack has given sufficient conditions in 32] under which the inclusion of strict algebras over a 2-monad into pseudo algebras over the same 2-monad admits a left adjoint whose unit has components that are equivalences. In such cases, every pseudo algebra over the 2-monad is equivalent to a strict algebra over the 2 -monad.

Definition 9.1. Let $\phi: S \rightarrow T$ be a morphism of theories and let $X$ be a pseudo $T$-algebra with action $\Psi: T \rightarrow \operatorname{End}(X)$. Let $U X$ be the pseudo $S$-algebra which has $X$ as its underlying category and $S$ structure maps defined by $\Psi(\phi(w)): X^{n} \rightarrow$ $X$ for $w \in S(n)$. Defining $U$ analogously for morphisms and 2-cells of the 2category of pseudo $T$-algebras gives a strict 2-functor $U$ from the 2-category of pseudo $T$-algebras to the 2-category of pseudo $S$-algebras called the forgetful 2functor associated to $\phi$.

To show that the forgetful 2-functor associated to $\phi$ has a left bi-adjoint, we need to find a bi-universal arrow of the following type: given a pseudo $S$-algebra $X$ there should exist a pseudo $T$-algebra $R$ and a bi-universal arrow $\eta_{X}: X \rightarrow U R$ in the category of pseudo $S$-algebras. In the next definition I define this $R$.

Definition 9.2. Let $\phi: S \rightarrow T$ be a morphism of theories. Let $X$ be a pseudo $S$ algebra with action $\Psi: S \rightarrow \operatorname{End}(X)$. Let $T^{\prime}$ denote the free theory on the sequence of sets $T(0), T(1), \ldots$ underlying the theory $T$. The category $A l g^{\prime}$ is the category whose objects are small $T^{\prime}$-algebras and morphisms are morphisms of strict $T^{\prime}$ algebras. Let $O b j G r a p h^{\prime}$ be the collection of small directed graphs whose object sets are discrete $T^{\prime}$-algebras. Let MorGraph' be morphisms of directed graphs such that the object component of each morphism is a map of discrete algebras. Then $G r a p h^{\prime}$ is a category. The forgetful functor $V: A l g^{\prime} \rightarrow G r a p h^{\prime}$ has a by Freyd's Adjoint Functor Theorem. We denote this left adjoint by $V^{\prime}$. It is like taking the free category on a directed graph, except the resulting category is also a $T^{\prime}$-algebra. The objects of the underlying directed graph of $V^{\prime} Y$ and the objects of the directed graph $Y$ are the same. the Let $O b j R_{G^{\prime}}$ be the (discrete) free $T^{\prime}$-algebra on the discrete category $\operatorname{Obj} X$ and let $M o r R_{G^{\prime}}$ be the collection of the following arrows: 
(1) For every $n \in \mathbf{N}$, for all words $w \in T(n), w_{1} \in T\left(m_{1}\right), \ldots, w_{n} \in T\left(m_{n}\right)$, and for all objects $A_{1}^{1}, \ldots, A_{m_{1}}^{1}, A_{1}^{2}, \ldots, A_{m_{2}}^{2}, \ldots, A_{1}^{n}, \ldots, A_{m_{n}}^{n} \in \operatorname{Obj}_{G^{\prime}}$ there is an arrow

$c_{w, w_{1}, \ldots, w_{n}}\left(A_{1}^{1}, \ldots, A_{m_{n}}^{n}\right): w \circ\left(w_{1}, \ldots, w_{n}\right)\left(A_{1}^{1}, \ldots, A_{m_{n}}^{n}\right) \rightarrow$ $w\left(w_{1}\left(A_{1}^{1}, \ldots, A_{m_{1}}^{1}\right), \ldots, w_{n}\left(A_{1}^{n}, \ldots, A_{m_{n}}^{n}\right)\right)$

and an arrow

$c_{w, w_{1}, \ldots, w_{n}}^{-1}\left(A_{1}^{1}, \ldots, A_{m_{n}}^{n}\right): w\left(w_{1}\left(A_{1}^{1}, \ldots, A_{m_{1}}^{1}\right), \ldots, w_{n}\left(A_{1}^{n}, \ldots, A_{m_{n}}^{n}\right)\right) \rightarrow$ $w \circ\left(w_{1}, \ldots, w_{n}\right)\left(A_{1}^{1}, \ldots, A_{m_{n}}^{n}\right)$. Here $w \circ\left(w_{1}, \ldots, w_{n}\right)$ is the composition in the original theory $T$. The target $w\left(w_{1}\left(A_{1}^{1}, \ldots, A_{m_{1}}^{1}\right), \ldots, w_{n}\left(A_{1}^{n}, \ldots, A_{m_{n}}^{n}\right)\right)$ is the result of composing in the free theory and applying it to the $A$ 's in the free algebra.

(2) For every $A \in \operatorname{Obj}_{G^{\prime}}$ there is an arrow $I_{A}: \operatorname{Id}(A) \rightarrow A$ and an arrow $I_{A}^{-1}: A \rightarrow I d(A)$. Here $I d$ is the unit of the original theory $T$.

(3) For every word $w \in T(m)$, for every function $f:\{1, \ldots, m\} \rightarrow\{1, \ldots, n\}$, and for all objects $A_{1}, \ldots, A_{n} \in \operatorname{Obj}_{G^{\prime}}$ there is an arrow $s_{w, f}\left(A_{1}, \ldots, A_{n}\right): w_{f}\left(A_{1}, \ldots, A_{n}\right) \rightarrow w\left(A_{f 1}, \ldots, A_{f m}\right)$ and an arrow $s_{w, f}^{-1}\left(A_{1}, \ldots, A_{n}\right): w\left(A_{f 1}, \ldots, A_{f m}\right) \rightarrow w_{f}\left(A_{1}, \ldots, A_{n}\right)$. The substituted word $w_{f}$ is the substituted word in the original theory $T$. The target $w\left(A_{f 1}, \ldots, A_{f m}\right)$ is the result of substituting in $w$ in the free theory and then evaluating on the $A$ 's.

(4) For every word $w \in S(n)$ and objects $A_{1}, \ldots, A_{n}$ of $X$ there is an arrow $\rho_{w}^{\eta}\left(A_{1}, \ldots A_{n}\right): \Psi(w)\left(A_{1}, \ldots, A_{n}\right) \rightarrow \phi(w)\left(A_{1}, \ldots, A_{n}\right)$

and an arrow $\rho_{w}^{\eta-1}\left(A_{1}, \ldots A_{n}\right): \phi(w)\left(A_{1}, \ldots, A_{n}\right) \rightarrow \Psi(w)\left(A_{1}, \ldots, A_{n}\right)$.

(5) Include also all elements of $M o r X$.

Then $R_{G^{\prime}}$ is an object of $G r a p h^{\prime}$. Now we apply $V^{\prime}$ to $R_{G^{\prime}}$ and we get a category $R^{\prime}$ which is a $T^{\prime}$-algebra. The objects of $R_{G^{\prime}}$ and $R^{\prime}$ are the same.

Let $K$ be the smallest congruence with the following properties:

(1) All of the relations necessary to make the coherence arrows (including $\rho_{w}^{\eta}$ ) into natural transformations belong to $K$. For example, if $A, B \in \operatorname{Obj}^{\prime}$ and $f: A \rightarrow B$ is a morphism of $R^{\prime}$, then the relation $I_{A} \circ f=I d(f) \circ I_{B}$ belongs to $K$.

(2) All of the relations necessary to make the coherence arrows (including $\rho_{w}^{\eta}$ ) into isos are in $K$. For example, for every $A \in O b j R^{\prime}$ the relations $I_{A} \circ I_{A}^{-1}=$ $1_{A}$ and $I_{A}^{-1} \circ I_{A}=1_{A}$ are in $K$.

(3) All of the relations listed in the definition of pseudo algebra above belong to $K$, where the objects range over the objects of $R^{\prime}$.

(4) The original composition relations in the category $X$ belong to $K$.

(5) The coherence diagrams necessary to make the inclusion $\eta_{X}: X \rightarrow U R$ into a morphism of pseudo $S$-algebras are in $K$. Note that these coherences will involve the arrows $\rho_{w}^{\eta}\left(A_{1}, \ldots, A_{n}\right): \Psi(w)\left(A_{1}, \ldots, A_{n}\right) \rightarrow \phi(w)\left(A_{1}, \ldots, A_{n}\right)$ for $w \in S(n)$ and objects $A_{1}, \ldots, A_{n} \in \operatorname{Obj} X$.

(6) If the relations $f_{1}=g_{1}, \ldots, f_{n}=g_{n}$ are in $K$ and $w \in T^{\prime}(n)$, then the relation $w\left(f_{1}, \ldots, f_{n}\right)=w\left(g_{1}, \ldots g_{n}\right)$ is also in $K$.

Next mod out $R^{\prime}$ by the congruence $K$ to obtain the quotient category $R$ called the free pseudo $T$-algebra on the pseudo $S$-algebra $X$ associated to $\phi$. We do not 
use a capital greek letter to denote the $T$ action on $R$. Rather we write the words directly.

In all of the following lemmas in this section we use the notation just introduced in Definition 9.2

Lemma 9.1. In the notation of the previous definition, the free pseudo T-algebra $R$ on the pseudo $S$-algebra $X$ associated to $\phi$ is a pseudo T-algebra.

Proof: $\quad$ First we note that $R$ is a (strict) $T^{\prime}$-algebra. The functor from the word $w \in T^{\prime}(n)$ induces a functor on the quotient by relation 6 . and the composition and identities in $T^{\prime}$ are preserved. The action has the coherence isos required of a pseudo $T$-algebra because of the arrows we threw in. The coherence isos satisfy the required coherence diagrams because of relations 1 . and 2 . Hence $R$ is a pseudo $T$-algebra.

Lemma 9.2. The inclusion functor denoted $\eta_{X}: X \rightarrow U R$ is a morphism of pseudo $S$-algebras.

Proof: The inclusion is a functor because of relation 4. It is a morphism of pseudo $S$-algebras because for all $w \in S(n)$ the natural transformation $\rho_{w}^{\eta}$ : $\eta_{X} \circ \Psi(w) \Rightarrow \phi(w)\left(\eta_{X}, \ldots, \eta_{X}\right)$ satisfies the required coherences by the relations in 1. and 5 .

Lemma 9.3. For every pseudo $T$-algebra $D$ and every morphism $H: X \rightarrow U D$ of pseudo $S$-algebras, there exists a morphism $H^{\prime}: R \rightarrow D$ of pseudo $T$-algebras such that

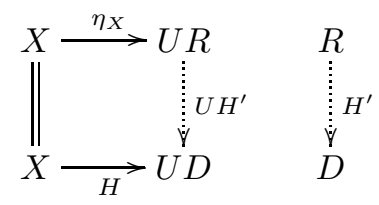

commutes.

Proof: $\quad$ Let $\Phi$ denote the action of $T$ on $D$. As above, $\Psi$ denotes the action of $S$ on $X$ and we suppress the capital greek letter when denoting the action of $T$ on $R$. Note that $D$ is a strict $T^{\prime}$ algebra and we can therefore apply the forgetful 2-functor $V: A l g^{\prime} \rightarrow G r a p h^{\prime}$ to it. We also use $\Phi$ to denote the action of $T^{\prime}$ on $D$. To construct the morphism $H^{\prime}$, I define a morphism $H_{0}^{\prime}: R_{G^{\prime}} \rightarrow V D$ in $G r a p h^{\prime}$, which induces a morphism $H_{1}^{\prime}: R^{\prime} \rightarrow D$ of $A l g^{\prime}$ by the definition of the left adjoint to $V$. Then I show that $H_{1}^{\prime}$ preserves the congruence $K$ and therefore induces a functor $H^{\prime}: R \rightarrow D$. Lastly I show that $H^{\prime}$ is a morphism of pseudo $T$-algebras such that the desired diagram commutes.

I now define a morphism $H_{0}^{\prime}: R_{G^{\prime}} \rightarrow V D$ in Graph'. Defining $H_{0}^{\prime} A:=$ $H A$ for $A \in \operatorname{ObjX}$ induces a map $H_{0}^{\prime}: \operatorname{Obj} R_{G^{\prime}} \rightarrow \operatorname{Obj} D$ of discrete $T^{\prime}$ algebras. For $f \in M$ or $X$ define $H_{0}^{\prime} f:=H f$. For every $w \in S(n)$ and objects $A_{1}, \ldots, A_{n} \in \operatorname{Obj} X$ let $H_{0}^{\prime}$ map the arrows $\rho_{w}^{\eta}\left(A_{1}, \ldots A_{n}\right): \Psi(w)\left(A_{1}, \ldots, A_{n}\right) \rightarrow$ $\phi(w)\left(A_{1}, \ldots, A_{n}\right)$ to the coherence isos $\rho_{w}^{H}\left(A_{1}, \ldots A_{n}\right): H\left(\Psi(w)\left(A_{1}, \ldots, A_{n}\right)\right) \rightarrow$ $\Phi(\phi(w))\left(H A_{1}, \ldots, H A_{n}\right)$. Note that the source and target of $\rho_{w}^{H}\left(A_{1}, \ldots A_{n}\right)$ are equal to $H_{0}^{\prime}\left(\Psi(w)\left(A_{1}, \ldots, A_{n}\right)\right)$ and $H_{0}^{\prime}\left(\phi(w)\left(A_{1}, \ldots, A_{n}\right)\right)$ respectively. Let $H_{0}^{\prime}$ map the other coherence arrows 1 . through 3 . to the analogous ones in $M$ or D with 
$H_{0}$ applied to sources and targets. Thus we have defined a morphism $H_{0}^{\prime}: R_{G^{\prime}} \rightarrow$ $V D$ in Graph'.

The morphism $H_{0}^{\prime}: R_{G^{\prime}} \rightarrow V D$ in Graph induces a morphism $H_{1}^{\prime}: R^{\prime} \rightarrow D$ of $A l g^{\prime}$ by the definition of the left adjoint to $V$. I claim that $H_{1}^{\prime}$ preserves the congruence $K$. It suffices to check the relations 1 . through 6 . I verify them in order of the list above.

(1) These are satisfied because the analogous arrows for $D$ and $H$ are natural transformations and $H_{1}^{\prime}$ maps coherence arrows to coherence arrows.

(2) These are satisfied because the analogous arrows for $D$ and $H$ are isos and $H_{1}^{\prime}$ maps coherence arrows to coherence arrows.

(3) The target category $D$ is a pseudo $T$-algebra so these are satisfied.

(4) The functor $H$ preserves the relations of the category $X$ and $H_{1}^{\prime}$ is defined in terms of $H$, which implies that these are satisfied.

(5) These are satisfied because $\rho_{w}^{H}$ satisfies the coherences and $H_{1}^{\prime}\left(\rho_{w}^{\eta}\right)=\rho_{w}^{H}$.

(6) This is by induction. The base case is showing 1 . through 5 . as was just done. Suppose the relations $f_{1}=g_{1}, \ldots, f_{n}=g_{n}$ are in $K$ and $H_{1}^{\prime} f_{i}=H_{1}^{\prime} g_{i}$ for all $i=1, \ldots, n$. That is our induction hypothesis. Then $H_{1}^{\prime}\left(w\left(f_{1}, \ldots, f_{n}\right)\right)=\Phi(w)\left(H_{1}^{\prime}\left(f_{1}\right), \ldots, H_{1}^{\prime}\left(f_{n}\right)\right)$ since $H_{1}^{\prime}$ is a morphism of $T^{\prime}$-algebras $=\Phi(w)\left(H_{1}^{\prime} g_{1}, \ldots, H_{1}^{\prime} g_{n}\right)$ by induction hypothesis $=H_{1}^{\prime}\left(w\left(g_{1}, \ldots, g_{n}\right)\right)$ since $H_{1}^{\prime}$ is a morphism of $T^{\prime}$-algebras.

Thus $H_{1}^{\prime}\left(w\left(f_{1}, \ldots, f_{n}\right)\right)=H_{1}^{\prime}\left(w\left(g_{1}, \ldots, g_{n}\right)\right)$ and $H_{1}^{\prime}$ satisfies this relation.

Since $H_{1}^{\prime}$ satisfies the relations, we conclude that $H_{1}^{\prime}: R^{\prime} \rightarrow D$ induces a functor $H^{\prime}: R \rightarrow D$ such that $H_{1}^{\prime}=H^{\prime} \circ Q$ where $Q: R^{\prime} \rightarrow R$ is the projection functor onto the quotient category. The functor $H^{\prime}: R \rightarrow D$ is a morphism of strict $T^{\prime}$-algebras because for $w \in T^{\prime}(n), A_{1}, \ldots, A_{n} \in \operatorname{Obj} R$, and for morphisms $f_{1}, \ldots, f_{n} \in \operatorname{Mor} R$ we have

$H^{\prime}\left(w\left(A_{1}, \ldots, A_{n}\right)\right)=H_{1}^{\prime}\left(w\left(A_{1}, \ldots, A_{n}\right)\right)$

$=\Phi(w)\left(H_{1}^{\prime} A_{1}, \ldots, H_{1}^{\prime} A_{n}\right)$

$=\Phi(w)\left(H^{\prime} A_{1}, \ldots, H^{\prime} A_{n}\right)$ since $H_{1}^{\prime}$ and $H^{\prime}$ agree on objects.

We also have

$H^{\prime}\left(w\left(f_{1}, \ldots, f_{n}\right)\right)=H_{1}^{\prime}\left(w\left(f_{1}, \ldots, f_{n}\right)\right)$

$=\Phi(w)\left(H_{1}^{\prime} f_{1}, \ldots, H_{1}^{\prime} f_{n}\right)$

$=\Phi(w)\left(H^{\prime} f_{1}, \ldots, H^{\prime} f_{n}\right)$, where $H_{1}^{\prime}$ is actually applied to representatives of

$w\left(f_{1}, \ldots, f_{n}\right), f_{1}, \ldots, f_{n}$. Hence $H^{\prime}$ is a morphism of strict $T^{\prime}$-algebras and also a morphism of pseudo $T$-algebras, since $T(n) \subseteq T^{\prime}(n)$ although this inclusion is not necessarily a map of theories. According to these two demonstrations, the coherence 2-cells for the morphism $H^{\prime}$ of pseudo $T$-algebras are just identities.

I claim that

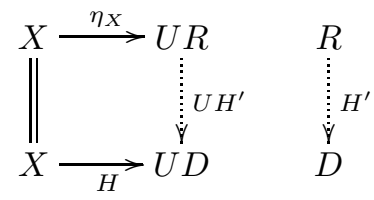

commutes. It is sufficient to check this for the underlying functors and the coherence 2-cells. The underlying functor of $H^{\prime}$ is the same as the underlying functor of $U H^{\prime}$. Let $A \in O b j X$. Then $U H^{\prime} \circ \eta_{X}(A)=U H^{\prime}(A)=H^{\prime} A=H A$. Similarly, for $f \in M$ or $X$ we have $U H^{\prime} \circ \eta_{X}(f)=U H^{\prime}(f)=H^{\prime} f=H f$. Hence the diagram 
commutes. The coherence 2-cells also commute because $H^{\prime}\left(\rho_{w}^{\eta}\right)=\rho_{w}^{H}$ and because the coherence 2-cells of $H^{\prime}$ are identities.

Lemma 9.4. The inclusion morphism $\eta_{X}: X \rightarrow U R$ is a bi-universal arrow from $X$ to the forgetful 2-functor.

Proof: $\quad$ Let $D$ be a pseudo $T$-algebra. Let $M_{\text {or }}(X, U D)$ denote the category of morphisms of pseudo $S$-algebras from $X$ to $U D$. Let $\operatorname{Mor}_{T}(R, D)$ denote the category of morphisms of pseudo $T$-algebras from $R$ to $D$. Let $\phi: M_{T} r_{T}(R, D) \rightarrow$ $M \operatorname{ror}_{S}(X, U D)$ be the functor defined by $H^{\prime} \mapsto U H^{\prime} \circ \eta_{X}$ and $\gamma \mapsto U \gamma * i_{\eta_{X}}$. Define a functor $\psi: \operatorname{Mor}_{S}(X, U D) \rightarrow \operatorname{Mor}_{T}(R, D)$ as follows. For $H \in \operatorname{ObjMor}_{S}(X, U D)$ let $\psi H:=H^{\prime}$ where $H^{\prime}: R \rightarrow D$ is the morphism of pseudo $T$ algebras constructed in the previous lemma.

If $H, J \in \operatorname{ObjMor}_{S}(X, U D)$ and $\beta: H \Rightarrow J$ is a 2-cell in the 2-category of pseudo $S$-algebras, define $\psi(\beta)=\beta^{\prime}: H^{\prime} \Rightarrow J^{\prime}$ inductively as follows. If $A \in \operatorname{ObjX}$ then

$\beta^{\prime} A:=\beta A: H A=H^{\prime} A \rightarrow J^{\prime} A=J A$. If $w \in T^{\prime}(n)$ and $\beta^{\prime}$ is already defined for $A_{1}, \ldots, A_{n} \in \operatorname{Obj} R$, then $\beta^{\prime}\left(w\left(A_{1}, \ldots, A_{n}\right)\right):=\Phi(w)\left(\beta^{\prime} A_{1}, \ldots, \beta^{\prime} A_{n}\right)$. The following inductive proof shows that $\beta^{\prime}: H^{\prime} \Rightarrow J^{\prime}$ is a natural transformation. For $f \in M$ or $X$ the naturality of $\beta^{\prime}$ is guaranteed by the naturality of $\beta: H \Rightarrow J$. The naturality of $\beta^{\prime}$ for the coherence isos thrown into the category $R$ during its construction follows because $H^{\prime}$ and $J^{\prime}$ take coherence isos of $R$ to analogous ones in $D$ and the coherences isos in $D$ are natural. That concludes the base case for the induction. Now suppose $\beta^{\prime}$ is natural for morphisms $f_{i} \in \operatorname{Mor}_{R}\left(A_{i}, B_{i}\right)$ for $i=1, \ldots, n$ and $w \in T^{\prime}(n)$. Then

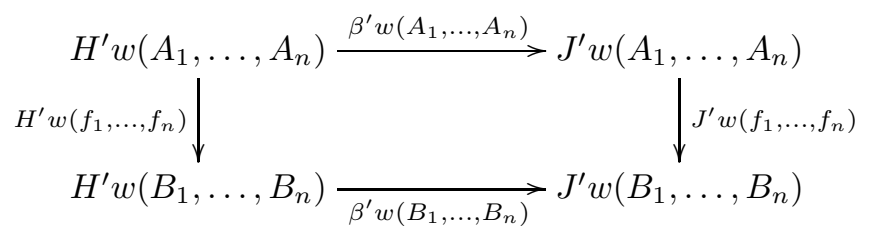

commutes because $w$ commutes with everything in the diagram by definition and because we apply the functor $\Phi(w)$ to each of the individual naturality diagrams for $f_{i}: A_{i} \rightarrow B_{i}$ and $i=1, \ldots, n$. Hence $\beta^{\prime}$ is natural for any morphism in $R$ by this inductive proof. Moreover, the natural transformation commutes appropriately with $\rho^{H^{\prime}}$ and $\rho^{J^{\prime}}$ because they are trivial and $\beta^{\prime}\left(w\left(A_{1}, \ldots, A_{n}\right)=\Phi(w)\left(\beta^{\prime} A_{1}, \ldots, \beta^{\prime} A_{n}\right)\right.$. Hence $\psi(\beta)=\beta^{\prime}$ is a 2-cell in the 2-category of pseudo $T$-algebras.

It is routine to check inductively that the assignment $\psi: \operatorname{Mor}_{S}(X, U D) \rightarrow$ $\operatorname{Mor}_{T}(R, D)$ preserves identities and compositions and is thus a functor.

I claim that $\psi$ is a right adjoint for $\phi$. By the previous lemma $\phi \circ \psi(H)=H$ for all $H \in \operatorname{ObjMor}_{S}(X, U D)$. One easily sees that $\phi \circ \psi(\beta)=\beta$ for all $\beta \in$ $\operatorname{MorMor}_{S}(X, U D)$. Hence the counit $\mu: \phi \circ \psi \Rightarrow 1_{M_{\text {or }}(X, U D)}$ is the identity natural transformation, which is of course a natural isomorphism. Next I define a unit $\theta: 1_{\operatorname{Mor}_{T}(R, D)} \Rightarrow \psi \circ \phi$. For $J^{\prime} \in M_{o_{T}}(R, D)$ let $H^{\prime}:=\psi \circ \phi\left(J^{\prime}\right)$. Recall that $H^{\prime}$ is strict, i.e. $\rho^{H^{\prime}}$ is trivial, while $J^{\prime}$ may not be strict. I define a 2-cell $\theta\left(J^{\prime}\right): J^{\prime} \rightarrow H^{\prime}=\psi \circ \phi\left(J^{\prime}\right)$ in the category of pseudo $T$-algebras inductively. For $A \in \operatorname{Obj} X \subseteq \operatorname{Obj} R$ set $\theta\left(J^{\prime}\right)(A):=1_{J^{\prime} A}$. Suppose $w \in T^{\prime}(n)$ and $\theta\left(J^{\prime}\right)$ is already defined for $A_{1}, \ldots, A_{n} \in \operatorname{Obj} R$. Then define $\theta\left(J^{\prime}\right)\left(w\left(A_{1}, \ldots, A_{n}\right)\right.$ : $J^{\prime}\left(w\left(A_{1}, \ldots, A_{n}\right)\right) \rightarrow H^{\prime}\left(w\left(A_{1}, \ldots, A_{n}\right)\right)$ by 
$\Phi(w)\left(\theta\left(J^{\prime}\right) A_{1}, \ldots, \theta\left(J^{\prime}\right) A_{n}\right) \circ \rho_{w}^{J^{\prime}}\left(A_{1}, \ldots, A_{n}\right)$. An inductive proof, similar to the one above but also using the naturality of $\rho_{w}^{J^{\prime}}$, shows that $\theta\left(J^{\prime}\right)$ is a natural transformation and commutes with $\rho^{J^{\prime}}$ and $\rho^{H^{\prime}}$ appropriately, i.e. $\theta\left(J^{\prime}\right): J^{\prime} \Rightarrow H^{\prime}$ is a 2 -cell. It is also iso by induction. The assignment $J^{\prime} \mapsto \theta\left(J^{\prime}\right)$ is natural by an inductive argument that uses the diagram in the definition of 2-cell in the 2-category of pseudo $T$-algebras. Hence $\theta: 1_{M r_{T}(R, D)} \Rightarrow \psi \circ \phi$ is a natural isomorphism. If one can show that $\theta$ and $\mu$ satisfy the triangular identities, then one can conclude that $\psi$ is a right adjoint for $\phi$

I claim that the unit $\theta$ and the counit $\mu$ satisfy the triangular identities. First I show that

$$
\psi \stackrel{\theta * i_{\psi}}{\Longrightarrow} \psi \circ \phi \circ \psi \stackrel{i_{\psi} * \mu}{\Longrightarrow} \psi
$$

is the identity natural transformation $i_{\psi}: \psi \Rightarrow \psi$. Let $H \in \operatorname{ObjMor}_{S}(X, U D)$. Then

$\left(i_{\psi} * \mu\right) \odot\left(\theta * i_{\psi}\right)(H)=\psi\left(\mu_{H}\right) \circ \theta_{\psi H}$ by definition $=\theta_{\psi H}$ since $\mu_{H}$ is trivial.

But $\theta_{\psi H}=\theta(\psi H)$ is the trivial 2-cell $\psi H \Rightarrow \psi H$ because $\psi H$ is a strict morphism of pseudo $T$-algebras, i.e. $\rho_{w}^{\psi H}$ is trivial. Hence (51) is $i_{\psi}: \psi \Rightarrow \psi$. Next I show that

$$
\phi \stackrel{i_{\phi} * \theta}{\Longrightarrow} \phi \circ \psi \circ \phi \stackrel{\mu * i_{\phi}}{\Longrightarrow} \phi
$$

is the identity natural transformation $i_{\phi}: \phi \Rightarrow \phi$. Let $J^{\prime} \in \operatorname{ObjMor}_{T}(R, D)$. Then $\left(\mu * i_{\phi}\right) \odot\left(i_{\phi} * \theta\right)\left(J^{\prime}\right)=\mu_{\phi J^{\prime}} \circ \phi\left(\theta_{J^{\prime}}\right)$ by definition

$=\phi\left(\theta_{J^{\prime}}\right)$ since $\mu_{\phi J^{\prime}}$ is trivial

$=\theta_{J^{\prime}} * i_{\eta_{X}}$ by definition.

But $\theta_{J^{\prime}} * i_{\eta_{X}}$ is the trivial 2-cell $\phi\left(J^{\prime}\right)=J^{\prime} \circ \eta_{X} \rightarrow J^{\prime} \circ \eta_{X}$ because $\theta_{J^{\prime}}(A)=$ $\theta\left(J^{\prime}\right)(A)=1_{J^{\prime} A}$ for all $A \in \operatorname{Obj} X$ and $\eta_{X}: X \rightarrow R$ is the inclusion functor. Hence (52) is the identity natural transformation $i_{\phi}: \phi \Rightarrow \phi$. Thus the unit and counit satisfy the triangular identities and $\psi$ is a right adjoint for $\phi$. Moreover, $\phi$ is an equivalence because the unit and counit are natural isomorphisms. We conclude that $\eta_{X}: X \rightarrow U R$ is a bi-universal arrow from $X$ to the 2 -functor $U$.

Remark 9.1. Although it is not necessary, we can construct the factorising 2-cell $\nu^{\prime}$ as follows. Let $H: X \rightarrow U D$ be a morphism of pseudo $S$-algebras. Then $\psi(H)=H^{\prime}$ satisfies

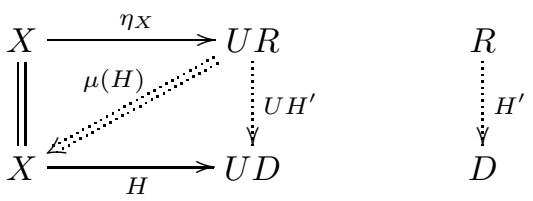

and $\mu(H)$ is the identity 2-cell. Suppose $\bar{H}^{\prime}: R \rightarrow D$ is another morphism of pseudo $T$-algebras and $\nu$ is a 2 -cell as follows.

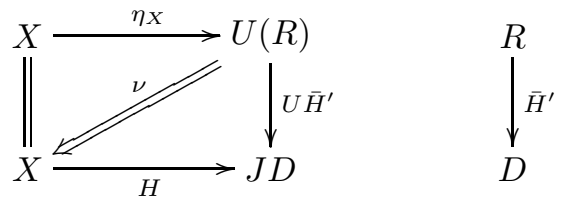


Define a 2-cell $\nu^{\prime}: \bar{H}^{\prime} \Rightarrow H^{\prime}$ as follows. For $A \in \operatorname{Obj} X \subseteq R, \nu^{\prime} A:=\nu A$. If $w \in T^{\prime}(n)$ and $\nu^{\prime}$ is already defined for $A_{1}, \ldots, A_{n} \in \operatorname{Obj} R$, then

$\nu^{\prime}\left(w\left(A_{1}, \ldots, A_{n}\right)\right):=\Phi(w)\left(\nu^{\prime} A_{1}, \ldots, \nu^{\prime} A_{n}\right) \circ \rho^{\bar{H}^{\prime}}\left(A_{1}, \ldots, A_{n}\right)$. By induction $\nu^{\prime}$ is a natural transformation. It also commutes with $\rho^{H^{\prime}}$ and $\rho^{\bar{H}^{\prime}}$ appropriately by construction. Hence $\nu^{\prime}$ is a 2-cell in the 2-category of pseudo T-algebras. By construction we see that

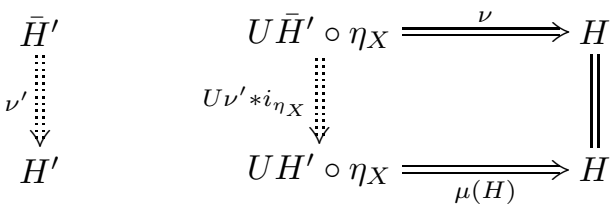

commutes. Such a 2-cell $\nu^{\prime}$ is unique by the requirement that (53) commutes and by the commutivity with $\rho^{\bar{H}^{\prime}}$ and $\rho^{H^{\prime}}$ required of 2-cells $\bar{H}^{\prime} \Rightarrow H^{\prime}$. More precisely, the commutivity of (53) says that $\nu^{\prime} A=\nu A$ for all $A \in \operatorname{Obj} X$ and the appropriate commutivity with $\rho^{\bar{H}^{\prime}}$ and $\rho^{H^{\prime}}$ specifies what $\nu^{\prime}$ does to objects of the form $w\left(A_{1}, \ldots, A_{n}\right)$ for $A_{1}, \ldots, A_{n} \in \operatorname{ObjR}$. If $\nu$ is iso, then so is $\nu^{\prime}$ by the construction and the fact that $\rho^{\bar{H}^{\prime}}$ is iso.

Theorem 9.5. The bi-universal arrows $\eta_{X}: X \rightarrow U F X$ define a strict 2-natural transformation $\eta: 1_{\mathcal{X}} \Rightarrow U \circ F$, where $\mathcal{X}$ is the 2-category of pseudo S-algebras.

Proof: $\quad$ Recall that the counits $\mu$ for the bi-universal arrows $\eta_{X}$ are all trivial as indicated on page 102 in Lemma 9.4 In the proof of Theorem 8.13 on page 95 the bi-universal arrows $\eta_{X}: X \rightarrow U F X$ are made into a pseudo natural transformation by defining $\tau_{f}:=\mu_{X, F Y}\left(\eta_{Y} \circ f\right)$ for $f: X \rightarrow Y$. We see that $\tau_{f}$ is trivial because $\mu_{X, F Y}$ is trivial. Hence $\eta$ is strictly 2 -natural.

Theorem 9.6. Let $S$ and $T$ be theories and $\phi: S \rightarrow T$ a morphism of theories. Then the forgetful 2-functor $U$ associated to $\phi$ from the 2-category of small pseudo $T$-algebras to the 2-category of small pseudo $S$-algebras has a left bi-adjoint denoted $F$. Moreover, this pseudo functor $F$ is actually a strict 2-functor.

Proof: $\quad$ For every pseudo $S$-algebra $X$ there exists a pseudo $T$-algebra $R$ and a bi-universal arrow $\eta_{X}: X \rightarrow U R$ by the Lemma 9.4 This guarantees the existence of a left bi-adjoint by Theorem 8.13

We can prove that $F$ is strict by inspecting its coherence isos constructed in the general theory of Theorem 8.13. Let $\mathcal{X}$ be the 2-category of pseudo $S$-algebras, $\mathcal{A}$ be the 2-category of pseudo $T$-algebras, and let $G:=U: \mathcal{A} \rightarrow \mathcal{X}$ be the forgetful 2 -functor. For any pseudo $S$-algebra $X \in \operatorname{Obj} \mathcal{X}$, we define $F X$ to be the free pseudo $T$-algebra $R$ on the pseudo $S$-algebra $X$ associated to the morphism of theories $\phi: S \rightarrow T$. The co-unit $\mu$ for the bi-universal $\eta_{X}: X \rightarrow U R$ is the identity as was seen in Lemma 9.4 The pseudo functor $U=G$ is actually a strict 2 -functor, so $\delta^{G}$ and $\gamma^{G}$ are identity natural transformations. After inspecting diagram (28) on page 86 we see that $\delta_{*}^{F}$ must be trivial because $\left(\delta_{F X *}^{G}\right)^{-1} * i_{\eta_{X}}$ and $\mu_{X, F X}\left(\eta_{X} \circ 1_{X}\right)=\mu\left(\eta_{X} \circ 1_{X}\right)$ are trivial. Hence $F$ preserves identites.

Similarly, each of the 2-cells in diagram (29) on page 87 is trivial, and therefore their composition is trivial. After inspecting diagram (30) on page 87 we see that $\gamma_{f, g}^{F}$ must also be trivial because both the horizontal top and bottom arrows are trivial. Therefore $F$ preserves compositions. 
Since $F$ preserves compositions and identities, it is a strict 2-functor.

Theorem 9.6 can be sharpened. Let $\mathcal{A}$ denote the 2 -category of pseudo $T$-algebras and let $\mathcal{X}$ denote the 2-category of pseudo $S$-algebras. Then the equivalence of categories $\operatorname{Mor}_{\mathcal{A}}(F X, A) \rightarrow \operatorname{Mor}_{\mathcal{X}}(X, U A)$ implicit in Theorem 9.6] is strictly 2natural in each variable. However, it can be shown that a left 2-adjoint does not exist in specific cases. The equivalence in the other direction $\operatorname{Mor}_{\mathcal{X}}(X, U A) \rightarrow$ $\operatorname{Mor}_{\mathcal{A}}(F X, A)$ in Theorem 9.6] is not strictly 2-natural in each variable. In fact, there is an example where there does not exist an equivalence $\operatorname{Mor}_{\mathcal{X}}(X, U A) \rightarrow$ $\operatorname{Mor}_{\mathcal{A}}(F X, A)$ which is strictly 2-natural in each variable, even after replacing $F$ by another bi-adjoint $F^{\prime}$. Counterexamples will be given after presenting Theorem 9.7 which is a sharper version of Theorem 9.6

Theorem 9.7. Let $S$ and $T$ be theories. Let $U: \mathcal{A} \rightarrow \mathcal{X}$ be the forgetful 2functor associated to a morphism $S \rightarrow T$ of theories. Let $F$ denote the left biadjoint to $U$ introduced in Theorem $\left[9.6\right.$. Then the equivalence of categories $\phi_{X, A}$ : $\operatorname{Mor}_{\mathcal{A}}(F X, A) \rightarrow \operatorname{Mor}_{\mathcal{X}}(X, U A)$ from Theorem 9.6 defined by $\phi_{X, A}(f):=U f \circ \eta_{X}$ is strictly 2 -natural in each variable.

Proof: The universal arrow $\eta_{X}: X \rightarrow U F X$ is the inclusion morphism. The functor $\phi_{X, A}: \operatorname{Mor}_{\mathcal{A}}(F X, A) \rightarrow \operatorname{Mor}_{\mathcal{X}}(X, U A)$ is defined by $\phi_{X, A}(f):=U f \circ \eta_{X}$ as in Lemma 8.9. The functor $\phi_{X, A}$ is an equivalence of categories for all $X \in O b j \mathcal{X}$ and all $A \in O \operatorname{Obj} \mathcal{A}$ because $\eta_{X}$ is a bi-universal arrow. The coherence isos $\tau^{\prime}$ for the pseudo naturality of $\phi_{-, A}$ are defined on page 80 in terms of some trivial 2-cells, $\gamma^{G}$, and $\tilde{\tau}$, where $\tilde{\tau}$ is the coherence iso for $\eta$. But $\gamma^{G}$ is trivial for $G=U$ because $U$ is a strict 2-functor. The coherence iso $\tilde{\tau}$ is also trivial because $\eta$ is a strict 2 -natural transformation. Hence $\tau^{\prime}$ is also trivial and $\phi_{-, A}$ is strictly 2-natural, i.e. $\phi$ is 2 -natural in the first variable.

The coherence isos $\tau$ for $\phi_{X,-}$ are defined on page 84 for morphisms $k: A \rightarrow A^{\prime}$ by $\tau_{A, A^{\prime}}(k): e \mapsto \gamma_{e, k}^{G} * i_{\eta_{X}}$. But $G=U$ is a strict functor and $\gamma^{G}$ is trival, hence $\tau$ is also trivial. Therefore $\phi_{X,-}$ is strictly 2-natural, i.e. $\phi$ is 2 -natural in the second variable. We conclude that $X, A \mapsto \phi_{X, A}$ is strictly 2 -natural in each variable.

Before proving that Theorem 9.6 cannot be further improved to a left 2-adjoint, we need a theorem which states that one can change a morphism of pseudo $T$ algebras in a specific way and still have a morphism of pseudo $T$-algebras.

Theorem 9.8. Let $X, Y$ be pseudo T-algebras and $H: X \rightarrow Y$ a morphism of pseudo T-algebras. Suppose that $J_{0}(x) \in \operatorname{Obj} Y$ and $\alpha_{0}(x): J_{0}(x) \rightarrow H(x)$ is an isomorphism for each $x \in O b j X$. Then there exists a morphism $J: X \rightarrow Y$ of pseudo T-algebras whose object function is $J_{0}$ and there exists an iso 2-cell $\alpha: J \rightarrow$ $H$ of pseudo $T$-algebras such that $\alpha(x)=\alpha_{0}(x)$ for all $x \in O b j X$. Moreover, such $J$ and $\alpha$ are unique.

Proof: $\quad$ For $x \in \operatorname{Obj} X$ define $J(x):=J_{0}(x)$ and $\alpha(x):=\alpha_{0}(x)$. For a morphism $f: x_{1} \rightarrow x_{2}$ of $X$ define $J(f):=\alpha\left(x_{2}\right)^{-1} \circ H(f) \circ \alpha\left(x_{1}\right)$. One easily sees that $J$ is a functor and $\alpha$ is natural transformation from $J$ to the functor underlying $H$.

For $w \in T(n)$ let $\rho_{w}^{H}: H \circ \Phi(w) \Rightarrow \Psi \circ(H, \ldots, H)$ denote the coherence isomorphism for $H$, where $\Phi$ denotes the action of the theory $T$ on $X$ and $\Psi$ denotes the action of the theory $T$ on $Y$. Define a natural isomorphism $\rho_{w}^{J}: J \circ \Phi(w) \Rightarrow$ $\Psi \circ(J, \ldots, J)$ by the following diagram. 


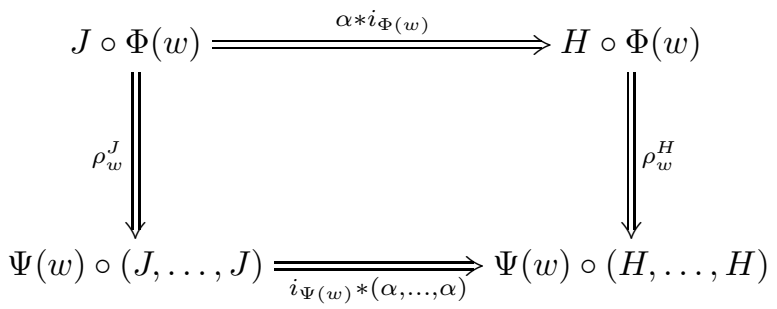

In other words $\rho_{w}^{J}:=i_{\Psi(w)} *\left(\alpha^{-1}, \ldots, \alpha^{-1}\right) \odot \rho_{w}^{H} \odot \alpha * i_{\Phi(w)}$. This is a natural transformation because it consists of horizontal and vertical compositions of natural transformations.

I claim that $\rho_{w}^{J}$ satisfies the coherence diagrams required to make $J$ a morphism of pseudo $T$-algebras. One can prove the commutivity of any $J$ coherence diagram from the commutivity of the analogous $H$ coherence diagram by using the following procedure. First one draws the commutative $H$ coherence diagram and then one circumscribes it with the analogous $J$ coherence diagram. Next one draws the obvios isomorphisms between respective $J$ and $H$ vertices. All of the resulting inner diagrams commute because of the interchange law, because of the definition of $\rho_{w}^{J}$, or because of the diagram for $H$. We present the substitution diagram to clarify the process. Let $f:\{1, \ldots, m\} \rightarrow\{1, \ldots, n\}$ be a function and $w \in T(m)$.

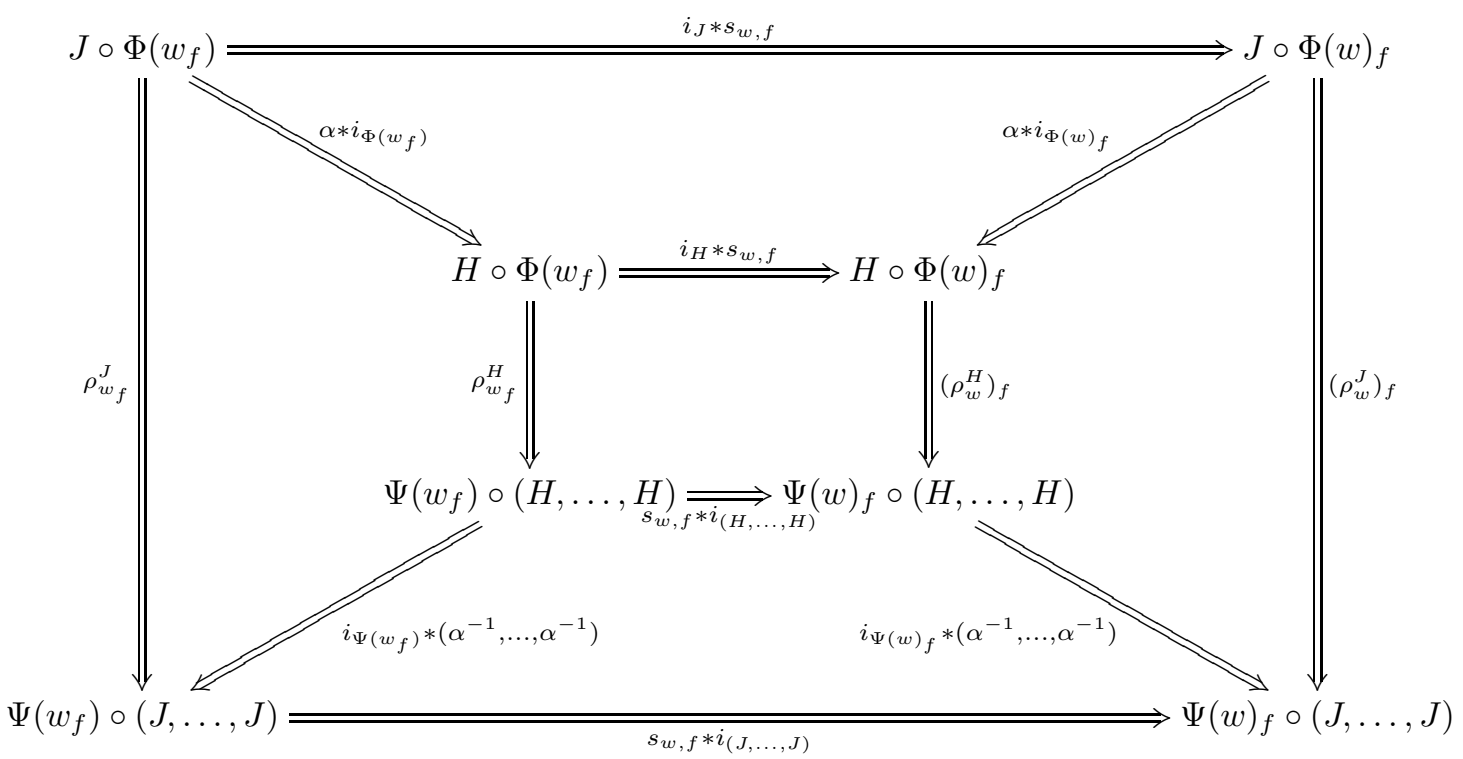

The top and bottom squares commute because of the interchange law. The left and right squares commute because of the definitions of $\rho_{w_{f}}^{J}$ and $\rho_{w}^{J}$. The innermost square commutes because $H$ is a morphism of pseudo $T$-algebras. Hence the outer rectangle commutes and $J$ satisfies the substitution coherence diagram.

The other diagrams can be verified using the same procedure. The only subtlety in this procedure occurs in the right hand vertical composition of the composition axiom. We reproduce the right hand part of the diagram obtained by following the procedure mentioned above. 


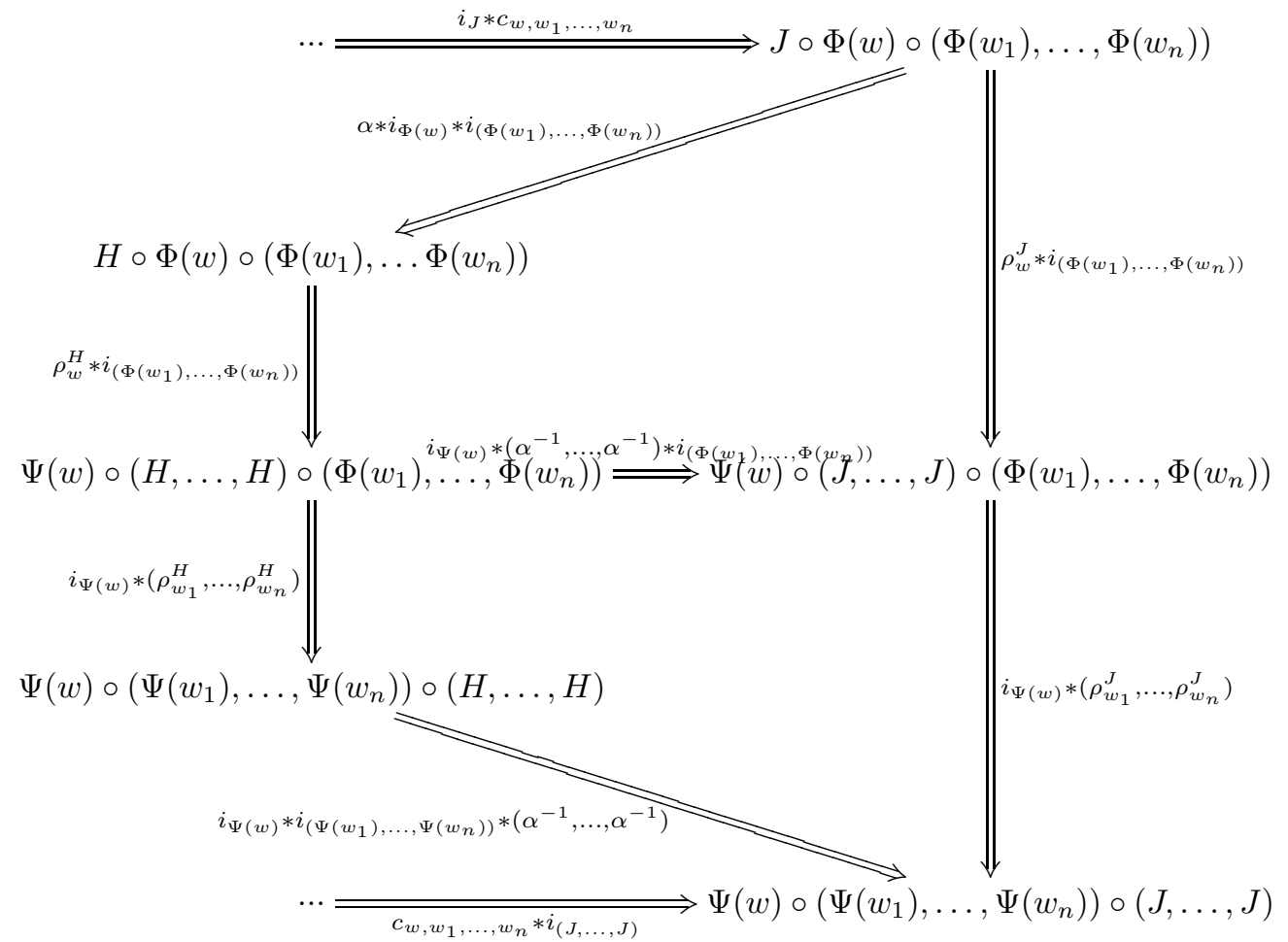

The upper right quadrilateral results from the diagram defining $\rho_{w}^{J}$ by horizontally composing with $i_{\left(\Phi\left(w_{1}\right), \ldots, \Phi\left(w_{n}\right)\right)}$. Then the upper right square commutes by iterated use of the interchange law.

The bottom right quadrilateral results from the defining diagrams of $\rho_{w_{1}}^{J}, \ldots, \rho_{w_{n}}^{J}$ by taking their product, horizontally composing with $i_{\left(\Psi\left(w_{1}\right), \ldots, \Psi\left(w_{n}\right)\right)}=\left(i_{\Psi\left(w_{1}\right)}, \ldots, i_{\Psi\left(w_{n}\right)}\right)$, and finally reversing one of the arrows. The commutivity then follows from the interchange law.

The other parts of the diagram are easily seen to commute, and we conclude that $J$ satisfies the composition coherence.

The commutivity of all of these coherence diagrams implies that $J$ is a morphism of pseudo $T$-algebras. We conclude that $\alpha$ is a 2-cell in the 2-category of pseudo $T$-algebras by looking at its defining diagram.

Suppose $J^{\prime}: X \rightarrow Y$ is a morphism of pseudo $T$-algebras and $\alpha^{\prime}: J^{\prime} \Rightarrow H$ is a 2-cell in the 2-category of pseudo $T$-algebras such that for all $x \in \operatorname{Obj} X$ we have $J^{\prime}(x)=J_{0}(x)$ and $\alpha^{\prime}(x)=\alpha_{0}(x)$. Then for a morphism $f: x_{1} \rightarrow x_{2}$ in $X$ the diagram

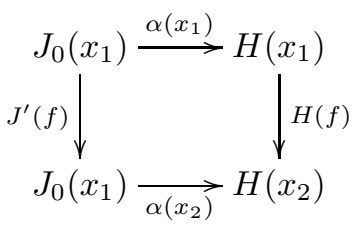


commutes. Hence $J^{\prime}(f)=\alpha\left(x_{2}\right)^{-1} \circ H(f) \circ \alpha\left(x_{1}\right)=J(f)$. For a word $w \in T(n)$, the diagram

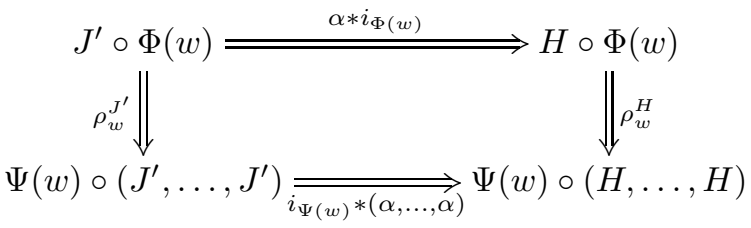

commutes. Hence $\rho_{w}^{J^{\prime}}=i_{\Psi(w)} *\left(\alpha^{-1}, \ldots, \alpha^{-1}\right) \odot \rho_{w}^{H} \odot \alpha * i_{\Phi(w)}=\rho_{w}^{J}$. We conclude $J^{\prime}=J$ as morphisms of pseudo $T$-algebras.

Lemma 9.9. The functor $\psi_{X, A}: \operatorname{Mor}_{\mathcal{X}}(X, U A) \rightarrow \operatorname{Mor}_{\mathcal{A}}(F X, A)$ in Theorem 9.6 is not strictly 2-natural in each variable.

Proof: Suppose $\psi$ is strictly 2-natural. Then for any morphism of pseudo $T$-algebras $J: F X \rightarrow F X$ the following diagram must commute.

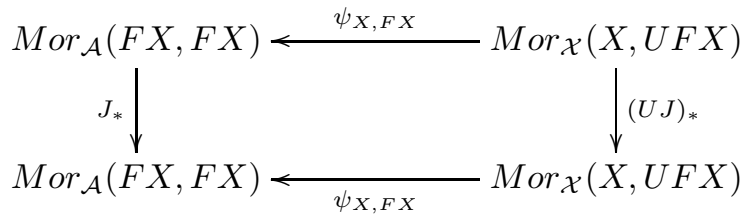

According to page 101 the output $\psi_{X, F X}(H)$ is always a strict morphism of pseudo $T$-algebras for all morphisms $H: X \rightarrow U F X$ of pseudo $S$-algebras. Let $a \in$ $\operatorname{Obj} F X$. Let $w$ be the trivial word in the theory $T$. Then $w\left(\psi_{X, F X}\left(\eta_{X}\right)(a)\right)$ is isomorphic to (but not equal to) $\psi_{X, F X}\left(\eta_{X}\right)(a)$ via a coherence isomorphism. By Theorem 9.8 we can construct from this data a morphism $J: F X \rightarrow F X$ of pseudo $T$-algebras such that $J\left(w\left(\psi_{X, F X}\left(\eta_{X}\right)(a)\right)\right)=\psi_{X, F X}\left(\eta_{X}\right)(a)$ and $J$ is the identity on all other objects. Chasing $\eta_{X}$ along diagram (54) from the top right corner, we see that $\psi_{X, F X}\left(U J \circ \eta_{X}\right)=J \circ \psi_{X, F X}\left(\eta_{X}\right)$ and $J \circ \psi_{X, F X}\left(\eta_{X}\right)$ must be strict because $\psi_{X, F X}\left(U J \circ \eta_{X}\right)$ is. But $J \circ \psi_{X, F X}\left(\eta_{X}\right)$ is not strict because it does not commute with the action of $w$ by the construction of $J$.

In fact, we present an example where there is no pseudo natural transformation $\psi$ as in Lemma 9.9 that is strictly 2-natural in the second variable, even after replacing $F$ by another left bi-adjoint to $U$. The reason is that our morphisms are not required to strictly commute with the action of the theories.

Example 19. Let $S$ be the trivial theory and let $T$ be the theory of commutative monoids. Let $\mathcal{X}$ be the 2-category of pseudo $S$-algebras and let $\mathcal{A}$ be the 2-category of pseudo $T$-algebras. Let $U: \mathcal{A} \rightarrow \mathcal{X}$ be the forgetful 2-functor associated to the trivial map of theories $S \rightarrow T$. Then there does not exist a left bi-adjoint $F^{\prime}: \mathcal{X} \rightarrow$ $\mathcal{A}$ which admits equivalences of categories $\psi_{X, A}^{\prime}: \operatorname{Mor}_{\mathcal{X}}(X, U A) \rightarrow \operatorname{Mor}_{\mathcal{A}}\left(F^{\prime} X, A\right)$ that are strictly 2-natural in the second variable.

Proof: $\quad$ First we prove that our constructed left bi-adjoint $F: \mathcal{X} \rightarrow \mathcal{A}$ does not admit equivalences $\psi_{X, A}^{\prime}$ that are strictly 2 -natural in the second variable. Suppose for each $X \in \operatorname{Obj} \mathcal{X}$ there exist equivalences $\psi_{X, A}^{\prime}: \operatorname{Mor}_{\mathcal{X}}(X, U A) \rightarrow \operatorname{Mor}_{\mathcal{A}}(F X, A)$ 
that are strictly natural in $A$, the second variable. Let $\phi_{X, A}^{\prime}$ be a functor such that $\phi_{X, A}^{\prime} \circ \psi_{X, A}^{\prime}$ and $\psi_{X, A}^{\prime} \circ \phi_{X, A}^{\prime}$ are naturally isomorphic to the respective identities.

Let $X$ be the pseudo $S$-algebra with only one object $*$ and no nontrivial morphisms. Let $A$ be the category of finite sets with a choice of disjoint union. This makes $A$ into a pseudo $T$-algebra.

I claim that there exists a morphism $H: X \rightarrow U A$ of pseudo $S$-algebras such that $\psi_{X, A}^{\prime}(H)(*) \neq \emptyset$. Suppose not. Then for every morphism $H: X \rightarrow U A$, we have $\psi_{X, A}^{\prime}(H)(w(*, \ldots, *)) \cong w(\emptyset, \ldots, \emptyset)=\emptyset$ and thus $\psi_{X, A}^{\prime}(H)$ is constant $\emptyset$. By the equivalence, every morphism $K: F X \rightarrow A$ of pseudo $T$-algebras is isomorphic to $\psi_{X, A}^{\prime} \circ \phi_{X, A}^{\prime}(K)$. This implies that $K$ must also be constant $\emptyset$. But this is a contradition, since there are nontrivial morphisms $F X \rightarrow A$. Thus there exists a morphism $H: X \rightarrow U A$ of pseudo $S$-algebras such that $\psi_{X, A}^{\prime}(H)(*) \neq \emptyset$.

I claim that there exists an object $x \in \operatorname{ObjFX}$ such that $\psi_{X, A}^{\prime}(H)(x) \neq H(*)$. Let $n \in \mathbb{N}$ be large enough that

$$
n \cdot\left|\psi_{X, A}^{\prime}(H)(*)\right|>|H(*)| .
$$

This is possible because $\left|\psi_{X, A}^{\prime}(H)(*)\right| \neq 0$ from above. Let $x=*+(*+(*+\cdots))$ where there are $n$ copies of $*$. Then $\left|\psi_{X, A}^{\prime}(H)(x)\right|=n \cdot\left|\psi_{X, A}^{\prime}(H)(*)\right|$ because $\psi_{X, A}^{\prime}(H)$ is a morphism of pseudo $T$-algebras and isomorphisms in $A$ are bijections of sets. Thus $\psi_{X, A}^{\prime}(H)(x) \neq H(*)$.

Let $J_{0}\left(\psi_{X, A}^{\prime}(H)(x)\right)$ be any set of the same cardinality as $\psi_{X, A}^{\prime}(H)(x)$ but not equal to $\psi_{X, A}^{\prime}(H)(x)$. Let $\alpha_{0}\left(\psi_{X, A}^{\prime}(H)(x)\right): J_{0}\left(\psi_{X, A}^{\prime}(H)(x)\right) \rightarrow \psi_{X, A}^{\prime}(H)(x)$ be a bijection. Let $J_{0}(a)=a$ for all $a \in O b j A$ such that $a \neq \psi_{X, A}^{\prime}(H)(x)$. Then by Theorem 9.8 there exists a morphism $J: A \rightarrow A$ of pseudo $T$-algebras which is the identity except on the object $\psi_{X, A}^{\prime}(H)(x)$. In particular $J(H(*))=H(*)$ because $H(*) \neq \psi_{X, A}^{\prime}(H)(x)$ from above.

The 2-naturality in the second variable implies that

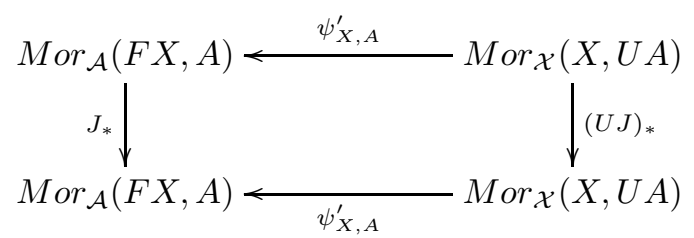

commutes, i.e. $J \circ \psi_{X, A}^{\prime}(H)=\psi_{X, A}^{\prime}(U J \circ H)$. But $U J \circ H=H$ because $J(H(*))=$ $H(*)$. Hence $J \circ \psi_{X, A}^{\prime}(H)=\psi_{X, A}^{\prime}(H)$. Evaluating this on $x$ gives

$$
J\left(\psi_{X, A}^{\prime}(H)(x)\right)=\psi_{X, A}^{\prime}(H)(x)
$$

which contradicts

$$
J\left(\psi_{X, A}^{\prime}(H)(x)\right) \neq \psi_{X, A}^{\prime}(H)(x) .
$$

Thus there cannot exist such a $\psi_{X, A}^{\prime}: \operatorname{Mor}_{\mathcal{X}}(X, U A) \rightarrow \operatorname{Mor}_{\mathcal{A}}(F X, A)$ and the reason is that we allow morphisms which do not strictly commute with the action of the theory.

Let $F^{\prime}: \mathcal{X} \rightarrow \mathcal{A}$ be any left bi-adjoint for $U: \mathcal{A} \rightarrow \mathcal{X}$. Suppose it admits equivalences of categories $\psi_{X, A}^{\prime}: \operatorname{Mor}_{\mathcal{X}}(X, U A) \rightarrow \operatorname{Mor}_{\mathcal{A}}\left(F^{\prime} X, A\right)$ that are strictly 2-natural in the second variable. Since $F$ and $F^{\prime}$ are left bi-adjoints for $U$, there exists for each $X$ a pseudo isomorphism $F X \rightarrow F X^{\prime}$ by the bi-universal arrow argument in Lemma 8.4 and Theorem 8.15] This pseudo isomorphism induces 
an equivalence of categories $\operatorname{Mor}_{\mathcal{A}}\left(F^{\prime} X, A\right) \rightarrow \operatorname{Mor}_{\mathcal{A}}(F X, A)$ which is strictly 2-natural in $A$. Composing this with $\psi_{X, A}^{\prime}$ gives an equivalence of categories $\operatorname{Mor}_{\mathcal{X}}(X, U A) \rightarrow \operatorname{Mor}_{\mathcal{A}}(F X, A)$ which is strictly 2-natural in $A$, the second variable. But it was shown above that such a 2 -natural equivalence cannot exist. Hence we have arrived at a contradiction and we conclude that $F^{\prime}$ does not admit equivalences $\psi_{X, A}^{\prime}: \operatorname{Mor}_{\mathcal{X}}(X, U A) \rightarrow \operatorname{Mor}_{\mathcal{A}}\left(F^{\prime} X, A\right)$ that are strictly 2-natural in the second variable.

We can build on the previous example to show that there does not exist a left 2 -adjoint to the forgetful 2 -functor in that situation.

Example 20. Let $S$ be the trivial theory and let $T$ be the theory of commutative monoids. Let $\mathcal{X}$ be the 2-category of pseudo $S$-algebras and let $\mathcal{A}$ be the 2-category of pseudo $T$-algebras. Let $U: \mathcal{A} \rightarrow \mathcal{X}$ be the forgetful 2-functor associated to the trivial map of theories $S \rightarrow T$. Then there does not exist a left 2-adjoint to $U$, i.e. there does not exist a 2-functor $F^{\prime}: \mathcal{X} \rightarrow \mathcal{A}$ which admits isomorphisms of categories $\phi_{X, A}: \operatorname{Mor}_{\mathcal{A}}\left(F^{\prime} X, A\right) \rightarrow \operatorname{Mor}_{\mathcal{X}}(X, U A)$ that are strictly 2-natural in each variable.

Proof: $\quad$ Suppose such a $\phi$ existed. Let $\psi_{X, A}:=\phi_{X, A}^{-1}$. Then $\psi_{X, A}$ is strictly 2 -natural in the second variable $A$ and is an equivalence of categories. But this is impossible by the previous example.

\section{Weighted Bicolimits of Pseudo T-algebras}

In this section I show that the 2-category of pseudo $T$-algebras has weighted bicolimits. The proof builds on the free pseudo $T$-algebra construction as well as the construction of strong colimits in the 2-category of small categories. The present construction does not capture pseudo colimits because of the equivalence of categories inherent in the construction of the free pseudo $T$-algebra. This equivalence comes from the fact that the morphisms of pseudo $T$-algebras are pseudo morphisms of pseudo $T$-algebras rather than strict ones. After proving that this 2-category admits bitensor products, we conclude that it admits all weighted bicolimits.

Theorem 10.1. The 2-category $\mathcal{C}$ of small pseudo T-algebras admits bicolimits.

Proof: $\quad$ Let $\mathcal{J}$ be a small 1-category and $F: \mathcal{J} \rightarrow \mathcal{C}$ a pseudo functor. In the following construction I use notation similar to the construction of the bi-universal arrow in the section on forgetful 2-functors.

First I define candidates $W \in O b j \mathcal{C}$ and $\pi: F \Rightarrow \Delta_{W}$. Let $T^{\prime}$ denote the free theory on the sequence of sets $T(0), T(1), \ldots$ underlying the theory $T$. Let $A l g^{\prime}$ be the category of small $T^{\prime}$-algebras. Let $\mathrm{Fraph}^{\prime}$ be the category of small directed graphs whose object sets are discrete $T^{\prime}$ algebras. Then there is a forgetful functor $A l g^{\prime} \rightarrow G r a p h^{\prime}$ and it has a left adjoint $V^{\prime}$ by Freyd's adjoint functor theorem.

Let $O b j R_{G^{\prime}}$ be the free (discrete) $T^{\prime}$ algebra on the set $\coprod_{j \in O b j \mathcal{J}} O b j F j$. Let $\operatorname{Mor} R_{G^{\prime}}$ be the collection of the following arrows:

(1) For every $n \in \mathbf{N}$, for all words $w \in T(n), w_{1} \in T\left(m_{1}\right), \ldots, w_{n} \in T\left(m_{n}\right)$, and for all objects $A_{1}^{1}, \ldots, A_{m_{1}}^{1}, A_{1}^{2}, \ldots, A_{m_{2}}^{2}, \ldots, A_{1}^{n}, \ldots, A_{m_{n}}^{n} \in O \operatorname{Obj} R_{G^{\prime}}$ there is an arrow

$$
c_{w, w_{1}, \ldots, w_{n}}\left(A_{1}^{1}, \ldots, A_{m_{n}}^{n}\right): w \circ\left(w_{1}, \ldots, w_{n}\right)\left(A_{1}^{1}, \ldots, A_{m_{n}}^{n}\right) \rightarrow
$$


$w\left(w_{1}\left(A_{1}^{1}, \ldots, A_{m_{1}}^{1}\right), \ldots, w_{n}\left(A_{1}^{n}, \ldots, A_{m_{n}}^{n}\right)\right)$

and an arrow

$c_{w, w_{1}, \ldots, w_{n}}^{-1}\left(A_{1}^{1}, \ldots, A_{m_{n}}^{n}\right): w\left(w_{1}\left(A_{1}^{1}, \ldots, A_{m_{1}}^{1}\right), \ldots, w_{n}\left(A_{1}^{n}, \ldots, A_{m_{n}}^{n}\right)\right) \rightarrow$

$w \circ\left(w_{1}, \ldots, w_{n}\right)\left(A_{1}^{1}, \ldots, A_{m_{n}}^{n}\right)$. Here $w \circ\left(w_{1}, \ldots, w_{n}\right)$ is the composition in

the original theory $T$. The target $w\left(w_{1}\left(A_{1}^{1}, \ldots, A_{m_{1}}^{1}\right), \ldots, w_{n}\left(A_{1}^{n}, \ldots, A_{m_{n}}^{n}\right)\right)$ is the result of composing in the free theory and applying it to the $A$ 's in the free algebra.

(2) For every $A \in \operatorname{Obj}_{G^{\prime}}$ there is an arrow $I_{A}: \operatorname{Id}(A) \rightarrow A$ and an arrow $I_{A}^{-1}: A \rightarrow I d(A)$. Here $I d$ is the unit of the original theory $T$.

(3) For every word $w \in T(m)$, for every function $f:\{1, \ldots, m\} \rightarrow\{1, \ldots, n\}$, and for all objects $A_{1}, \ldots, A_{n} \in \operatorname{Obj}_{G^{\prime}}$ there is an arrow

$s_{w, f}\left(A_{1}, \ldots, A_{n}\right): w_{f}\left(A_{1}, \ldots, A_{n}\right) \rightarrow w\left(A_{f 1}, \ldots, A_{f m}\right)$

and an arrow

$s_{w, f}^{-1}\left(A_{1}, \ldots, A_{n}\right): w\left(A_{f 1}, \ldots, A_{f m}\right) \rightarrow w_{f}\left(A_{1}, \ldots, A_{n}\right)$. The substituted word $w_{f}$ is the substituted word in the original theory $T$. The target $w\left(A_{f 1}, \ldots, A_{f m}\right)$ is the result of substituting in $w$ in the free theory and then evaluating on the $A$ 's.

(4) For every word $w \in T(n), j \in O b j \mathcal{J}$, and objects $A_{1}, \ldots, A_{n}$ of $F j$ there is an arrow $\rho_{w}^{\pi_{j}}\left(A_{1}, \ldots, A_{n}\right): \Phi_{j}(w)\left(A_{1}, \ldots, A_{n}\right) \rightarrow w\left(A_{1}, \ldots, A_{n}\right)$ and an arrow $\left(\rho_{w}^{\pi_{j}}\right)^{-1}\left(A_{1}, \ldots, A_{n}\right): w\left(A_{1}, \ldots, A_{n}\right) \rightarrow \Phi_{j}(w)\left(A_{1}, \ldots, A_{n}\right)$, where $\Phi_{j}$ is the action of $T$ on the pseudo $T$-algebra $F j$.

(5) Include all elements of $\coprod_{j \in \mathcal{J}} \operatorname{Mor} F j$ in $M$ or $R_{G^{\prime}}$.

(6) For every morphism $f: i \rightarrow j$ of $\mathcal{J}$ and every $x \in O b j F i$ we include arrows $h_{(x, f)}$ and $h_{(x, f)}^{-1}$ as in the proof of Theorem 3.1

With these arrows, $R_{G^{\prime}}$ is an object of $G r a p h^{\prime}$. Now we apply the functor $V^{\prime}$ to the directed graph $R_{G^{\prime}}$ to get a category $R^{\prime}$ which is a $T^{\prime}$ algebra.

Let $K$ be the smallest congruence on the category $R^{\prime}$ with the following properties:

(1) All of the relations necessary to make the coherence arrows (including $\rho_{w}^{\pi_{j}}$ ) into natural transformations belong to $K$. For example, if $A, B \in \operatorname{Obj}^{\prime}$ and $f: A \rightarrow B$ is a morphism of $R^{\prime}$, then the relation $I_{A} \circ f=I d(f) \circ I_{B}$ belongs to $K$.

(2) All of the relations necessary to make the coherence arrows (including $\rho_{w}^{\pi_{j}}$ ) into isos are in $K$. For example, for every $A \in O b j R^{\prime}$ the relations $I_{A} \circ I_{A}^{-1}=$ $1_{A}$ and $I_{A}^{-1} \circ I_{A}=1_{A}$ are in $K$.

(3) All of the relations listed in the definition of pseudo algebra above belong to $K$, where the objects range over the objects of $R^{\prime}$.

(4) The original composition relations in each of the categories $F j$ belong to $K$ for all $j \in O b j \mathcal{J}$.

(5) The coherence diagrams necessary to make the inclusion $\pi_{j}: F j \rightarrow R^{\prime}$ into a morphism of pseudo $T$-algebras belong to $K$. Note that these coherences will involve the arrows $\rho_{w}^{\pi_{j}}\left(A_{1}, \ldots, A_{n}\right)$ for $w \in T(n)$.

(6) All of the relations in the proof of Theorem 3.1 are in $K$.

(7) If the relations $f_{1}=g_{1}, \ldots, f_{n}=g_{n}$ are in $K$ and $w \in T^{\prime}(n)$, then the relation $w\left(f_{1}, \ldots, f_{n}\right)=w\left(g_{1}, \ldots g_{n}\right)$ is also in $K$.

Next we $\bmod R^{\prime}$ out by $K$ and we get a pseudo $T$-algebra $R=: W \in O \operatorname{Obj} \mathcal{C}$. 
I define a pseudo natural transformation $\pi: F \Rightarrow \Delta_{W}$ as follows. For $j \in O b j \mathcal{J}$, define $\pi_{j}: F j \rightarrow W$ to be the inclusion functor. The functor $\pi_{j}$ is a morphism of pseudo $T$-algebras because of the relations we modded out by. Define $\tau_{i, j}(f)_{x}$ : $\pi_{i}(x) \rightarrow \pi_{j} \circ a_{f}(x)$ by $\tau_{i, j}(f)_{x}:=h_{(x, f)}$ as in the proof of Theorem of 3.1 Then $x \mapsto \tau_{i, j}(f)_{x}$ is a 2-cell $\pi_{i} \Rightarrow \pi_{j} \circ a_{f}$ in the 2-category of pseudo $T$-algebras because of the relations we modded out by and because of the work in the proof of Theorem 3.1 By an argument similar to Lemma 3.2 we conclude that $\pi: F \rightarrow \Delta_{W}$ is a pseudo natural transformation. The candidate for the bicolimit of $F$ is $W \in O b j \mathcal{C}$ with the pseudo cone $\pi: F \rightarrow \Delta_{W}$. This concludes the definition of the candidate for the bicolimit of $F$.

Let $V \in O b j \mathcal{C}$. Define the functor $\phi: \operatorname{Mor}_{\mathcal{C}}(W, V) \rightarrow P \operatorname{seudoCone}(F, V)$ by $b \mapsto b \circ \pi$ as before. We need to see that $\phi$ is an equivalence of categories.

Lemma 10.2. There is a functor $\psi: \operatorname{PseudoCone}(F, V) \rightarrow \operatorname{Mor}_{\mathcal{C}}(W, V)$.

Proof: $\quad$ First I define $\psi$ on objects. Let $\pi^{\prime}: F \Rightarrow \Delta_{V}$ be a pseudo natural transformation. From $\pi^{\prime}$ we get a map of sets

$$
\coprod_{j \in O b j \mathcal{J}} O b j F j \rightarrow O b j V
$$

which induces a map

$$
d: O b j R_{G^{\prime}} \rightarrow O b j V
$$

of discrete $T^{\prime}$ algebras. Define $d$ on arrows of $R_{G^{\prime}}$ as follows:

- $d g:=\pi_{j}^{\prime} g$ for all $g \in M o r F j$ and all $j \in O b j \mathcal{J}$

- $d h_{(x, f)}:=\tau_{i, j}^{\prime}(f)_{x}$ and $d h_{(x, f)}^{-1}:=\left(\tau_{i, j}^{\prime}(f)_{x}\right)^{-1}$ for $f: i \rightarrow j$ in $\mathcal{J}$ and $x \in \operatorname{ObjFi}$

- $d$ takes a coherence arrow in $R_{G^{\prime}}$ to the analogous coherence iso in $V$

- $d\left(\rho_{w}^{\pi_{j}}\right):=\rho_{w}^{\pi_{j}^{\prime}}$ where $\rho_{w}^{\pi_{j}^{\prime}}$ is the coherence iso of the morphism $\pi_{j}^{\prime}: F j \rightarrow V$ of pseudo $T$-algebras, and similarly $d\left(\left(\rho_{w}^{\pi_{j}}\right)^{-1}\right):=\left(\rho_{w}^{\pi_{j}^{\prime}}\right)^{-1}$.

This defines a morphism $d: R_{G^{\prime}} \rightarrow V$ of the category Graph', where part of the structure of the $T^{\prime}$-algebra $V$ is forgotten. The adjoint $G r a p h^{\prime} \rightarrow A l g^{\prime}$ to the forgetful functor gives us a morphism $R^{\prime} \rightarrow V$, which we also denote by $d$. Furthermore, $d: R^{\prime} \rightarrow V$ preserves the relations we want to impose. Hence $d$ induces a map $b: R \rightarrow V$ on the quotient and $d$ is a morphism of pseudo $T$ algebras. Note that the coherence isos of $b$ are trivial. This is how we define $\psi$ on objects: $\psi\left(\pi^{\prime}\right):=b$.

Let $\sigma, \sigma^{\prime} \in \operatorname{ObjPseudoCone}(F, V)$ and $\Xi: \sigma \rightsquigarrow \sigma^{\prime}$ be a morphism of PseudoCone $(F, V)$. Then define a 2-cell $\psi(\Xi): \psi(\sigma) \Rightarrow \psi\left(\sigma^{\prime}\right)$ by $\psi(\Xi)_{x}:=\Xi_{j}(x)$ for $x \in O b j F j$ and continue the definition inductively by

$$
\psi(\Xi)_{w\left(x_{1}, \ldots, x_{n}\right)}:=\Psi(w)\left(\psi(\Xi)_{x_{1}}, \ldots, \psi(\Xi)_{x_{n}}\right),
$$

where $\Psi$ is the action of $T$ on $V$. Another inductive argument shows that this assignment preserves compositions and identities.

Lemma 10.3. The composite functor $\phi \circ \psi: P \operatorname{seudoCone}(F, V) \rightarrow P$ seudoCone $(F, V)$ is the identity functor.

Proof: This is similar to Lemma 3.5 The only difference here is that one must prove that the coherence iso's for the morphism $\pi_{j}^{\prime}: F j \rightarrow V$ of pseudo $T$-algebras 
are the same as the coherence iso's for $\left(\phi \circ \psi\left(\pi^{\prime}\right)\right)_{j}$. But this is true because the coherence iso's of $\psi\left(\pi^{\prime}\right)$ are trivial.

Lemma 10.4. The composite functor $\psi \circ \phi: \operatorname{Mor}_{\mathcal{C}}(W, V) \rightarrow \operatorname{Mor}_{\mathcal{C}}(W, V)$ is naturally isomorphic to the identity functor.

Proof: I construct a natural isomorphism $\eta: 1_{M o r_{C}(W, V)} \Rightarrow \psi \circ \phi$. Let $b \in$ $\operatorname{ObjMor}_{\mathcal{C}}(W, V)$. We define $\eta_{b}=: \alpha$ inductively. For all $j \in \operatorname{Obj} \mathcal{J}$ and all $x \in$ $O b j F j \subseteq O b j W$ we have $\psi \circ \phi(b)(x)=b(x)$. Define

$$
\alpha_{x}: b(x) \rightarrow \psi \circ \phi(b)(x)
$$

to be the identity for such $x$. For $w \in T(n)$ and $x_{1}, \ldots, x_{n} \in \coprod_{j \in O b j \mathcal{J}} O b j F j$ define

$$
\alpha_{w\left(x_{1}, \ldots, x_{n}\right)}:=\rho_{w}^{b}\left(x_{1}, \ldots, x_{n}\right) .
$$

Now let $x_{1}, \ldots, x_{n} \in \operatorname{Obj} W$ and $w \in T(n)$. Suppose $\alpha_{x_{1}}, \ldots, \alpha_{x_{n}}$ are already defined. Then define

$$
\alpha_{w\left(x_{1}, \ldots, x_{n}\right)}: b\left(w\left(x_{1}, \ldots, x_{n}\right)\right) \rightarrow \psi \circ \phi(b)\left(w\left(x_{1}, \ldots, x_{n}\right)\right)
$$

to be the composition

$$
\begin{gathered}
b\left(w\left(x_{1}, \ldots, x_{n}\right)\right) \\
\downarrow \rho_{w}^{b}\left(x_{1}, \ldots, x_{n}\right) \\
\Psi(w)\left(b x_{1}, \ldots, b x_{n}\right) \\
\downarrow \Psi(w)\left(\alpha_{x_{1}}, \ldots, \alpha_{x_{n}}\right) \\
\Psi(w)\left(\psi \circ \phi(b) x_{1}, \ldots, \psi \circ \phi(b) x_{n}\right) .
\end{gathered}
$$

Then the assignment $x \mapsto \alpha_{x}$ is a 2-cell in the category of pseudo $T$-algebras because it is natural and commutes with the coherence isos of $b$ and $\psi \circ \phi(b)$ by an inductive argument (recall the coherence isos of $\psi \circ \phi(b)$ are trivial). An inductive argument also shows that $b \mapsto \eta_{b}$ is natural.

Lemma 10.5. The functor $\phi: \operatorname{Mor}_{\mathcal{C}}(W, V) \rightarrow P \operatorname{seudoCone}(F, V)$ defined by $b \mapsto$ $b \circ \pi$ is an equivalence of categories.

Proof: This follows immediately from the previous two lemmas.

Lemma 10.6. The object $W \in O b j \mathcal{C}$ and the pseudo cone $\pi: F \Rightarrow \Delta_{W}$ comprise a bicolimit of $F$.

Proof: This follows immediately from the previous lemma.

This completes the proof that the 2-category of small pseudo $T$-algebras admits bicolimits.

Lemma 10.7. The 2-category $\mathcal{C}$ of pseudo T-algebras admits bitensor products.

Proof: $\quad$ Let $J$ be a category and $F$ a pseudo $T$-algebra. First define an object $R_{G^{\prime}}$ of $G r a p h^{\prime}$. Let $O b j R_{G^{\prime}}$ be the free discrete $T^{\prime}$-algebra on the set $O b j J \times O b j F$, where $T^{\prime}$ is the free theory on $T$. Let $M o r R_{G^{\prime}}$ be the collection of the following arrows. 
(1) For every $n \in \mathbf{N}$, for all words $w \in T(n), w_{1} \in T\left(m_{1}\right), \ldots, w_{n} \in T\left(m_{n}\right)$, and for all objects $A_{1}^{1}, \ldots, A_{m_{1}}^{1}, A_{1}^{2}, \ldots, A_{m_{2}}^{2}, \ldots, A_{1}^{n}, \ldots, A_{m_{n}}^{n} \in \operatorname{Obj}_{G^{\prime}}$ there is an arrow

$c_{w, w_{1}, \ldots, w_{n}}\left(A_{1}^{1}, \ldots, A_{m_{n}}^{n}\right): w \circ\left(w_{1}, \ldots, w_{n}\right)\left(A_{1}^{1}, \ldots, A_{m_{n}}^{n}\right) \rightarrow$ $w\left(w_{1}\left(A_{1}^{1}, \ldots, A_{m_{1}}^{1}\right), \ldots, w_{n}\left(A_{1}^{n}, \ldots, A_{m_{n}}^{n}\right)\right)$

and an arrow

$c_{w, w_{1}, \ldots, w_{n}}^{-1}\left(A_{1}^{1}, \ldots, A_{m_{n}}^{n}\right): w\left(w_{1}\left(A_{1}^{1}, \ldots, A_{m_{1}}^{1}\right), \ldots, w_{n}\left(A_{1}^{n}, \ldots, A_{m_{n}}^{n}\right)\right) \rightarrow$ $w \circ\left(w_{1}, \ldots, w_{n}\right)\left(A_{1}^{1}, \ldots, A_{m_{n}}^{n}\right)$. Here $w \circ\left(w_{1}, \ldots, w_{n}\right)$ is the composition in the original theory $T$. The target $w\left(w_{1}\left(A_{1}^{1}, \ldots, A_{m_{1}}^{1}\right), \ldots, w_{n}\left(A_{1}^{n}, \ldots, A_{m_{n}}^{n}\right)\right)$ is the result of composing in the free theory and applying it to the $A$ 's in the free algebra.

(2) For every $A \in O \operatorname{Obj} R_{G^{\prime}}$ there is an arrow $I_{A}: \operatorname{Id}(A) \rightarrow A$ and an arrow $I_{A}^{-1}: A \rightarrow I d(A)$. Here $I d$ is the unit of the original theory $T$.

(3) For every word $w \in T(m)$, for every function $f:\{1, \ldots, m\} \rightarrow\{1, \ldots, n\}$, and for all objects $A_{1}, \ldots, A_{n} \in \operatorname{Obj}_{G^{\prime}}$ there is an arrow $s_{w, f}\left(A_{1}, \ldots, A_{n}\right): w_{f}\left(A_{1}, \ldots, A_{n}\right) \rightarrow w\left(A_{f 1}, \ldots, A_{f m}\right)$ and an arrow $s_{w, f}^{-1}\left(A_{1}, \ldots, A_{n}\right): w\left(A_{f 1}, \ldots, A_{f m}\right) \rightarrow w_{f}\left(A_{1}, \ldots, A_{n}\right)$. The substituted word $w_{f}$ is the substituted word in the original theory $T$. The target $w\left(A_{f 1}, \ldots, A_{f m}\right)$ is the result of substituting in $w$ in the free theory and then evaluating on the $A$ 's.

(4) For every word $w \in T(n), j \in O b j J$, and objects $x_{1}, \ldots, x_{n}$ of $F$ there is an arrow $\rho_{w}^{\pi(j)}\left(\left(j, x_{1}\right), \ldots,\left(j, x_{n}\right)\right):\left(j, \Phi(w)\left(x_{1}, \ldots, x_{n}\right)\right) \rightarrow w\left(\left(j, x_{1}\right), \ldots,\left(j, x_{n}\right)\right)$ and an arrow $\left(\rho_{w}^{\pi(j)}\right)^{-1}: w\left(\left(j, x_{1}\right), \ldots,\left(j, x_{n}\right)\right) \rightarrow\left(j, \Phi(w)\left(x_{1}, \ldots, x_{n}\right)\right)$, where $\Phi$ is the structure map of the pseudo $T$-algebra $F$.

(5) Include all elements of $M o r J \times M o r F$ in $M o r R_{G^{\prime}}$.

With these arrows, $R_{G^{\prime}}$ is an object of $G r a p h^{\prime}$. Now we apply the free $T^{\prime}$-algebra functor to the directed graph $R_{G^{\prime}}$ to get a category $R^{\prime}$ which is a $T^{\prime}$ algebra. Let $K$ be the smallest congruence on the category $R^{\prime}$ with the following properties:

(1) All of the relations necessary to make the coherence arrows (including $\rho_{w}^{\pi(j)}$ ) into natural transformations belong to $K$. For example, if $A, B \in O b j R^{\prime}$ and $f: A \rightarrow B$ is a morphism of $R^{\prime}$, then the relation $I_{A} \circ f=I d(f) \circ I_{B}$ belongs to $K$.

(2) All of the relations necessary to make the coherence arrows (including $\rho_{w}^{\pi(j)}$ ) into isos are in $K$. For example, for every $A \in O b j R^{\prime}$ the relations $I_{A} \circ I_{A}^{-1}=$ $1_{A}$ and $I_{A}^{-1} \circ I_{A}=1_{A}$ are in $K$.

(3) All of the relations listed in the definition of pseudo algebra above belong to $K$, where the objects range over the objects of $R^{\prime}$.

(4) The original composition relations in the category $J \times F$ belong to $K$.

(5) For each $j \in J$, the coherence diagrams necessary to make the inclusion $F \rightarrow R^{\prime}, x \mapsto(j, x)$ into a morphism of pseudo $T$-algebras belong to $K$. Note that these coherences will involve the arrows $\rho_{w}^{\pi(j)}\left(\left(j, x_{1}\right), \ldots,\left(j, x_{n}\right)\right)$ : $\left(j, \Phi(w)\left(x_{1}, \ldots, x_{n}\right)\right) \rightarrow w\left(\left(j, x_{1}\right), \ldots,\left(j, x_{n}\right)\right)$. 
(6) For any $g: j_{1} \rightarrow j_{2}$ in $J$ and $x_{1}, \ldots, x_{n}$ in $F$ we include the relation

$$
\begin{aligned}
& \quad\left(j_{1}, \Phi(w)\left(x_{1}, \ldots, x_{n}\right)\right) \stackrel{\left(g, 1_{\Phi(w)\left(x_{1}, \ldots, x_{n}\right)}\right)}{\longrightarrow}\left(j_{2}, \Phi(w)\left(x_{1}, \ldots, x_{n}\right)\right) \\
& \rho_{w}^{\pi\left(j_{1}\right)}\left(x_{1}, \ldots, x_{n}\right) \downarrow \\
& w\left(\left(j_{1}, x_{1}\right), \ldots,\left(j_{1}, x_{n}\right)\right) \frac{\mid \rho_{w}^{\pi\left(j_{2}\right)}\left(x_{1}, \ldots, x_{n}\right)}{w\left(\left(g, x_{1}\right), \ldots,\left(g, x_{n}\right)\right)} w\left(\left(j_{2}, x_{1}\right), \ldots,\left(j_{2}, x_{n}\right)\right) .
\end{aligned}
$$

(7) If the relations $f_{1}=g_{1}, \ldots, f_{n}=g_{n}$ are in $K$ and $w \in T^{\prime}(n)$, then the relation $w\left(f_{1}, \ldots, f_{n}\right)=w\left(g_{1}, \ldots g_{n}\right)$ is also in $K$.

Next we $\bmod R^{\prime}$ out by $K$ and we get a pseudo $T$-algebra $J * F \in O b j \mathcal{C}$. I define a functor $\pi: J \rightarrow \mathcal{C}(F, J * F)$ by

$$
\begin{aligned}
\pi(j)(x) & :=(j, x) \\
\pi(j)(f) & :=\left(1_{j}, f\right) \\
(\pi(g))_{x} & :=\left(g, 1_{x}\right)
\end{aligned}
$$

for $j \in O b j J, x \in O b j F, f \in M o r F$, and $g \in M o r J$. Then $\pi(j): F \rightarrow J * F$ is a morphism of pseudo $T$-algebras with coherence isos $\rho^{\pi(j)}$ and $\pi(g): \pi\left(j_{1}\right) \rightarrow \pi\left(j_{2}\right)$ is a 2-cell in the 2-category of pseudo $T$-algebras because of the relations. The relations also imply that $\pi$ is a functor.

I claim that $\pi$ induces an equivalence

$$
\begin{aligned}
\mathcal{C}(J * F, C) & \stackrel{\phi}{\longrightarrow} \operatorname{Cat}(J, \mathcal{C}(F, C)) \\
b & \mapsto \mathcal{C}(F, b) \circ \pi \\
\alpha & \mapsto \mathcal{C}(F, \alpha) * i_{\pi}
\end{aligned}
$$

of categories. Define a functor $\psi: \operatorname{Cat}(J, \mathcal{C}(F, C)) \rightarrow \mathcal{C}(J * F, C)$ as follows. For a functor $\sigma: J \rightarrow \mathcal{C}(F, C)$, we have a map of sets

$$
\begin{gathered}
O b j J \times O b j F \rightarrow O b j C \\
(j, x) \mapsto \sigma(j)(x)
\end{gathered}
$$

which induces a map $O b j R_{G^{\prime}} \rightarrow O b j C$ of discrete $T^{\prime}$-algebras satsifying

$$
\begin{gathered}
\psi(\sigma)(j, x):=\sigma(j)(x) \\
\psi(\sigma)\left(w\left(\left(j_{1}, x_{1}\right), \ldots,\left(j_{n}, x_{n}\right)\right)\right):=\Phi^{C}(w)\left(\sigma\left(j_{1}\right)\left(x_{1}\right), \ldots, \sigma\left(j_{n}\right)\left(x_{n}\right)\right)
\end{gathered}
$$

for $(j, x),\left(j_{1}, x_{1}\right), \ldots,\left(j_{n}, x_{n}\right) \in J \times F$. Define $\psi(\sigma)$ on arrows of $R_{G^{\prime}}$ by

$$
\begin{gathered}
\Psi(\sigma)\left(c_{w, w_{1}, \ldots, w_{n}}\left(A_{1}^{1}, \ldots, A_{m_{n}}^{n}\right)\right):=c_{w, w_{1}, \ldots, w_{n}}\left(\psi(\sigma)\left(A_{1}^{1}\right), \ldots, \psi(\sigma)\left(A_{m_{n}}^{n}\right)\right) \\
\psi(\sigma)\left(I_{A}\right):=I_{\psi(\sigma)\left(I_{A}\right)} \\
\psi(\sigma)\left(s_{w, f}\left(A_{1}, \ldots, A_{n}\right)\right):=s_{w, f}\left(\psi(\sigma)\left(A_{1}\right), \ldots, \psi(\sigma)\left(A_{n}\right)\right) \\
\psi(\sigma)(g, f):=\sigma\left(j_{2}\right)(f) \circ \sigma(g)_{x_{1}}=\sigma(g)_{x_{2}} \circ \sigma\left(j_{1}\right)(f)
\end{gathered}
$$

for $A_{\ell}^{k}, A, A_{i} \in O b j R_{G^{\prime}}, f: m \rightarrow n, g: j_{1} \rightarrow j_{2}$ in $J$ and $f: x_{1} \rightarrow x_{2}$ in $F$. We define $\psi(\sigma)$ similarly for $c_{w, w_{1}, \ldots, w_{n}}^{-1}, I_{A}^{-1}, s_{w, f}^{-1}$. Then $\psi(\sigma): R_{G^{\prime}} \rightarrow C$ is a morphism of $G r a p h^{\prime}$, which induces a morphism $R^{\prime} \rightarrow C$ in $A l g^{\prime}$. It preserves the relations and therefore induces a morphism $\psi(\sigma): J * F \rightarrow C$ of pseudo $T$-algebras on the quotient. This is actually a strict morphism of pseudo $T$-algebras. For a natural transformation $\Xi: \sigma \rightarrow \sigma^{\prime}$ define a 2-cell $\psi(\Xi): \psi(\sigma) \Rightarrow \psi\left(\sigma^{\prime}\right)$ inductively by

$$
\psi(\Xi)_{(j, x)}:=\left(\Xi_{j}\right)_{x}
$$


for $(j, x) \in O b j J \times O b j F$ and

$$
\psi(\Xi)_{w\left(A_{1}, \ldots, A_{n}\right)}:=\Phi^{C}(w)\left(\psi(\Xi)_{A_{1}}, \ldots, \psi(\Xi)_{A_{n}}\right)
$$

whenever $\psi(\Xi)_{A_{1}}, \ldots, \psi(\Xi)_{A_{n}}$ are already defined. From these definitions we can conclude that $\psi$ is a functor and $\phi \circ \psi=1_{C a t(J, \mathcal{C}(F, C))}$. For example,

$$
\begin{aligned}
(\phi \circ \psi(\sigma))(j)(x) & =(\psi(\sigma) \circ \pi(j))(x) \\
& =\psi(\sigma)(j, x) \\
& =\sigma(j)(x)
\end{aligned}
$$

and also

$$
\begin{aligned}
\left((\phi \circ \psi(\Xi))_{j}\right)_{x} & =\left(\left(\psi(\Xi) * i_{\pi}\right)_{j}\right)_{x} \\
& =\psi(\Xi)_{\pi(j)(x)} \\
& =\left(\Xi_{j}\right)_{x} .
\end{aligned}
$$

I construct a natural isomorphism $\eta: 1_{\mathcal{C}(J * F, C)} \Rightarrow \psi \circ \phi$. Let $b: J * F \rightarrow C$ be a morphism of pseudo $T$-algebras. We define $\eta_{b}=: \alpha$ inductively. For all $(j, x) \in O b j J \times O b j F$ we have

$$
\begin{aligned}
\psi \circ \phi(b)(j, x) & =\psi(\mathcal{C}(F, b) \circ \pi)(j, x) \\
& =(\mathcal{C}(F, b) \circ \pi)(j)(x) \\
& =(b \circ \pi(j))(x) \\
& =b(j, x) .
\end{aligned}
$$

Define

$$
\alpha_{(j, x)}: b(j, x) \rightarrow \psi \circ \phi(b)(j, x)
$$

to be the identity for $\operatorname{such}(j, x)$. For $w \in T(n)$ and $\left(j_{1}, x_{1}\right), \ldots,\left(j_{n}, x_{n}\right) \in \operatorname{Obj} J \times$ ObjF define

$$
\alpha_{w\left(\left(j_{1}, x_{1}\right), \ldots,\left(j_{n}, x_{n}\right)\right)}:=\rho_{w}^{b}\left(\left(j_{1}, x_{1}\right), \ldots,\left(j_{n}, x_{n}\right)\right) .
$$

For $A_{1}, \ldots, A_{n} \in O b j R_{G^{\prime}}=O b j J * F$ and $w \in T(n)$, define

$$
\alpha_{w\left(A_{1}, \ldots, A_{n}\right)}: b\left(w\left(A_{1}, \ldots, A_{n}\right)\right) \rightarrow \psi \circ \phi(b)\left(w\left(A_{1}, \ldots, A_{n}\right)\right)
$$

to be the composition

$$
\begin{gathered}
b\left(w\left(A_{1}, \ldots, A_{n}\right)\right) \\
\rho_{w}^{b}\left(A_{1}, \ldots, A_{n}\right) \\
\Psi(w)\left(b A_{1}, \ldots, b A_{n}\right) \\
\downarrow \begin{array}{l}
\Psi(w)\left(\alpha_{A_{1}}, \ldots, \alpha_{A_{n}}\right) \\
\Psi(w)\left(\psi \circ \phi(b) A_{1}, \ldots, \psi \circ \phi(b) A_{n}\right) .
\end{array}
\end{gathered}
$$

Then the assignment $x \mapsto \alpha_{x}$ is a 2-cell in the category of pseudo $T$-algebras because it is natural and commutes with the coherence isos of $b$ and $\psi \circ \phi(b)$ by an inductive argument (recall the coherence isos of $\psi \circ \phi(b)$ are trivial). An inductive argument also shows that $b \mapsto \eta_{b}$ is natural.

By Remark 2.3 this implies that $J * F$ is a bitensor product of $J$ and $F$.

Theorem 10.8. The 2-category $\mathcal{C}$ of pseudo T-algebras admits all weighted bicolimits. 
Proof: $\quad$ The 2-category $\mathcal{C}$ admits bicoproducts and bicoequalizers by Theorem 10.1 It admits bitensor products by the previous lemma. Hence by Theorem 2.5 it admits all weighted bicolimits.

\section{STACKS}

In this section we introduce the language of stacks in analogy to sheaves, since stacks generalize sheaves. A stack is a contravariant pseudo functor from a Grothendieck topology to a 2-category which takes Grothendieck covers to bilimits in the sense described below. The target 2-category is required to admit bilimits. We have shown that the 2-category of pseudo algebras over a theory admits bilimits, so we can speak of stacks of pseudo algebras. Some references for stacks are 12, [15], 17], 40], and [52].

Definition 11.1. A basis for a Grothendieck topology on a category $\mathcal{B}$ with pullbacks is a function $K$ which assigns to each object $B$ of $\mathcal{B}$ a collection of families of morphims with codomain $B$ such that

(1) If $g: B^{\prime} \rightarrow B$ is an isomorphism, then $\{g\} \in K(B)$

(2) If $\left\{g_{i}: B_{i} \rightarrow B \mid i \in I\right\} \in K(B)$, then for any morphism $g: D \rightarrow B$ the family of pullbacks of the $g_{i}$ along $g\left\{\pi_{2}: B_{i} \times{ }_{B} D \rightarrow D \mid i \in I\right\}$ is in $K(D)$

(3) If $\left\{g_{i}: B_{i} \rightarrow B \mid i \in I\right\} \in K(B)$ and $\left\{f_{i j}: D_{i j} \rightarrow B_{i} \mid j \in J_{i}\right\} \in K\left(B_{i}\right)$ for all $i$, then the composite family $\left\{g_{i} \circ f_{i j}: B_{i j} \rightarrow B \mid i \in I, j \in J_{i}\right\}$ is in $K(B)$.

The second axiom is called the stability axiom because it says that $K$ is stable under pullbacks. The third axiom is called the transitivity axiom. Often one refers to the basis as well as the category $\mathcal{B}$ as a Grothendieck topology. We follow this convention. Some authors call a Grothendieck topology a Grothendieck site. The elements of $K(B)$ are called Grothendieck covers.

Definition 11.2. Let $\mathcal{B}$ be a Grothendieck topology and $\mathcal{C}$ a concrete category. Then a $\mathcal{C}$-sheaf on $\mathcal{B}$ is a contravariant functor $G: \mathcal{B} \rightarrow \mathcal{C}$ which takes Grothendieck covers to limits, i.e. for any object $B$ of $\mathcal{B}$ and for any Grothendieck cover $\left\{g_{i}\right.$ : $\left.B_{i} \rightarrow B \mid i \in I\right\} \in K(B)$ the following diagram is an equalizer

$$
G(B) \stackrel{e}{\longrightarrow} \prod_{i \in I} G\left(B_{i}\right) \stackrel{p_{1}}{\underset{p_{2}}{\longrightarrow}} \prod_{i, j \in I} G\left(B_{i} \times_{B} B_{j}\right)
$$

where $e(a)=\left\{G\left(g_{i}\right) a\right\}_{i \in I}$ and $p_{1}\left(\left\{a_{k}\right\}_{k \in I}\right)_{i j}=G\left(\pi_{i j}^{1}\right) a_{i}$ and $p_{2}\left(\left\{a_{k}\right\}_{k \in I}\right)_{i j}=$ $G\left(\pi_{i j}^{2}\right) a_{j}$. Here $\pi_{i j}^{1}, \pi_{i j}^{2}$ are the morphisms in the pullback diagrams.

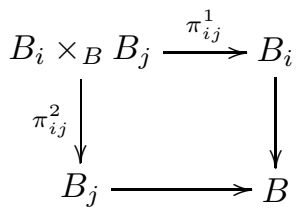

See [38 for a thorough discussion of Grothendieck topologies and sheaves. The diagram above is an equalizer if and only if it is exact. Usually one speaks of a $\mathcal{C}$-sheaf as a sheaf of objects of $\mathcal{C}$. For example, if $\mathcal{C}$ is the category of sets, then we speak of a sheaf of sets. Next we speak of stacks of categories and then generalize to stacks of objects with algebraic structure.

Let $C a t$ denote the 2-category of small categories. Suppose $\mathcal{B}$ is a Grothendieck topology. Let $G: \mathcal{B} \rightarrow C a t$ be a contravariant pseudo functor. Let $B$ be an object 
of $\mathcal{B}$ and $\left\{g_{i}: B_{i} \rightarrow B \mid i \in I\right\} \in K(B)$ a Grothendieck cover. Consider the diagram

$$
\prod_{i \in I} G\left(B_{i}\right) \underset{p_{2}}{\stackrel{p_{1}}{\longrightarrow}} \prod_{i, j \in I} G\left(B_{i} \times_{B} B_{j}\right) \underset{\frac{p_{12}}{-p_{23}}}{\stackrel{p_{23}}{\rightrightarrows}} \prod_{i, j, k \in I} G\left(B_{i} \times{ }_{B} B_{j} \times_{B} B_{k}\right)
$$

where the arrows are defined as

$$
\begin{gathered}
p_{1}\left(\left\{a_{k}\right\}_{k}\right)_{i j}:=G\left(\pi_{i j}^{1}\right) a_{i} \\
p_{2}\left(\left\{a_{k}\right\}_{k}\right)_{i j}:=G\left(\pi_{i j}^{2}\right) a_{j} \\
p_{12}\left(\left\{a_{\ell m}\right\}_{\ell m}\right)_{i j k}:=G\left(\pi_{i j k}^{12}\right) a_{i j} \\
p_{13}\left(\left\{a_{\ell m}\right\}_{\ell m}\right)_{i j k}:=G\left(\pi_{i j k}^{13}\right) a_{i k} \\
p_{23}\left(\left\{a_{\ell m}\right\}_{\ell m}\right)_{i j k}:=G\left(\pi_{i j k}^{23}\right) a_{j k} .
\end{gathered}
$$

Here $\pi_{i j k}^{12}, \pi_{i j k}^{13}, \pi_{i j k}^{23}$ are the morphisms in the triple fiber product as in the following commutative diagram from [52]. Here $B_{i j k}$ is the fibered product $B_{i} \times{ }_{B} B_{j} \times{ }_{B}$ $B_{k}$. The other fibered products are denoted similarly. The unlabelled arrows are $g_{i}, g_{j}, g_{k}$ from the Grothendieck cover.

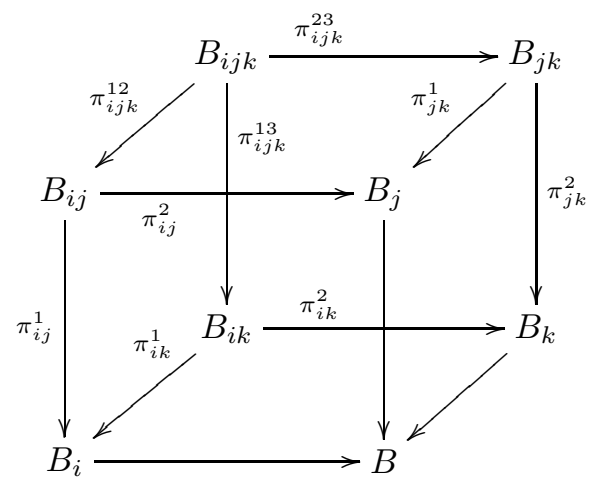

Every face in this diagram is a pullback square. The object $B_{i j k}$ is the limit of the diagram obtained from this one by deleting $B_{i j k}$ and the arrows emanating from it.

Diagram (56) can be interpreted as the image of a pseudo functor $F: \mathcal{J} \rightarrow C a t$ as follows. Let $\mathcal{J}$ be the free 1-category on the directed graph

$$
X \underset{f_{2}}{\stackrel{f_{1}}{\rightleftarrows}} Y \underset{f_{23}}{\stackrel{f_{12}}{=}} Z
$$


modded out by the relations below.
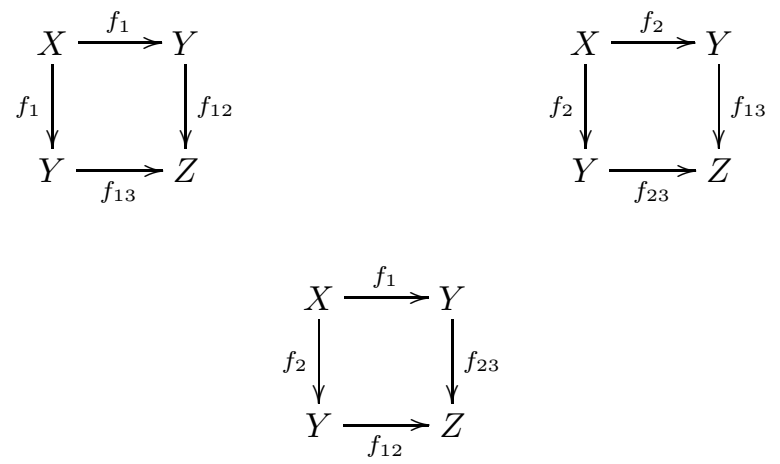

Define a pseudo functor $F: \mathcal{J} \rightarrow C$ at which takes diagram (57) to the diagram (56) and takes identity morphisms to identity morphisms. The pseudo functor $F$ is defined on all possible composites of nontrivial morphisms as:

$$
\begin{aligned}
& F\left(f_{12} \circ f_{1}\right)\left(\left\{a_{\ell}\right\}_{\ell}\right)_{i j k}:=G\left(\pi_{i j}^{1} \circ \pi_{i j k}^{12}\right) a_{i} \\
& F\left(f_{13} \circ f_{2}\right)\left(\left\{a_{\ell}\right\}_{\ell}\right)_{i j k}:=G\left(\pi_{i k}^{2} \circ \pi_{i j k}^{13}\right) a_{k} \\
& F\left(f_{23} \circ f_{1}\right)\left(\left\{a_{\ell}\right\}_{\ell}\right)_{i j k}:=G\left(\pi_{j k}^{1} \circ \pi_{i j k}^{23}\right) a_{j} .
\end{aligned}
$$

The identity coherence isos $\delta^{F}$ for $F$ are equalities because $F$ takes identity morphisms to identity morphisms. The coherence isos $\gamma^{F}$ for composites of non-identity morphisms are defined as tuples of the composition coherence isos for $G$. For example, the coherence iso $\gamma_{f_{1}, f_{12}}^{F}\left\{a_{\ell}\right\}_{\ell}: F\left(f_{12}\right) \circ F\left(f_{1}\right)\left\{a_{\ell}\right\}_{\ell} \rightarrow F\left(f_{12} \circ f_{1}\right)\left\{a_{\ell}\right\}_{\ell}$ is defined as

$$
\left\{\gamma_{\pi_{i j k}^{12}, \pi_{i j}^{1}}^{G} a_{i}\right\}_{i j k}:\left\{G\left(\pi_{i j k}^{12}\right) \circ G\left(\pi_{i j}^{1}\right) a_{i}\right\}_{i j k} \rightarrow\left\{G\left(\pi_{i j}^{1} \circ \pi_{i j k}^{12}\right) a_{i}\right\}_{i j k}
$$

The coherence isos $\gamma^{F}$ for composites involving one or more identity morphisms are defined to be equalities. For example, the coherence iso

$$
\gamma_{1_{X}, f_{1}}^{F}\left\{a_{\ell}\right\}_{\ell}: F\left(f_{1}\right) \circ F\left(1_{X}\right)\left\{a_{\ell}\right\}_{\ell} \rightarrow F\left(f_{1} \circ 1_{X}\right)\left\{a_{\ell}\right\}_{\ell}
$$

is equality. The coherence diagram in the pseudo functor unit axiom for $\delta^{F}$ is satisfied because of this definition. The coherence diagram in the pseudo functor composition axiom for $\gamma^{F}$ is satisfied because of the diagrams for $\gamma^{G}$ and also because of this definition. The coherence isos are also natural because $\mathcal{J}$ has no nontrivial 2-cells. Thus $F: \mathcal{J} \rightarrow$ Cat is a pseudo functor whose image is diagram (56). By a bilimit of diagram (56) we mean a bilimit of this functor $F$.

In the context of stacks there is a canonical candidate for the bilimit of $F$, namely $G(B)$. The candidate for the universal pseudo cone $\pi^{\prime}: \Delta_{G(B)} \Rightarrow F$ is defined on objects as follows.

$$
\begin{gathered}
\pi_{X}^{\prime}: G(B) \rightarrow \prod_{i} G\left(B_{i}\right) \\
\pi_{X}^{\prime}(a):=\left\{G\left(g_{i}\right) a\right\}_{i} \\
\pi_{Y}^{\prime}: G(B) \rightarrow \prod_{i, j} G\left(B_{i} \times_{B} B_{j}\right) \\
\pi_{Y}^{\prime}(a):=\left\{G\left(g_{i} \circ \pi_{i j}^{1}\right) a\right\}_{i j}
\end{gathered}
$$




$$
\begin{gathered}
\pi_{Z}^{\prime}: G(B) \rightarrow \prod_{i, j, k} G\left(B_{i} \times_{B} B_{j} \times_{B} B_{k}\right) \\
\pi_{Z}^{\prime}(a):=\left\{G\left(g_{i} \circ \pi_{i j}^{1} \circ \pi_{i j k}^{12}\right) a\right\}_{i j k}
\end{gathered}
$$

The coherence isos $\tau_{f}^{\prime}: F f \circ \pi_{S f}^{\prime} \Rightarrow \Delta_{G(B)}(f) \circ \pi_{T f}^{\prime}$ for the pseudo cone $\pi^{\prime}$ and nonidentity morphisms $f$ in $\mathcal{J}$ are defined in terms of $\gamma^{G}$. For example, for $f_{1}: X \rightarrow Y$ we have

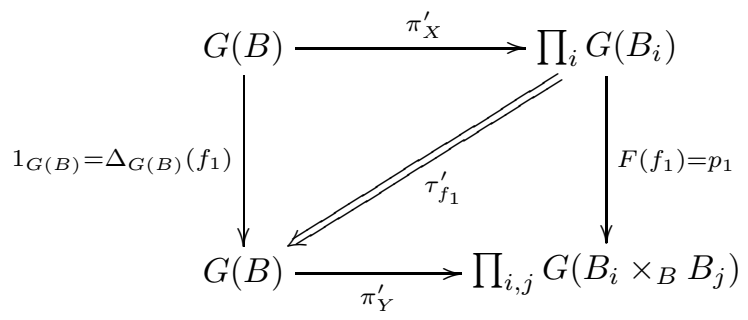

defined by $\tau_{f_{1}}^{\prime} a:=\left\{\gamma_{\pi_{i j}^{1}, g_{i}}^{G} a\right\}_{i j}:\left\{G\left(\pi_{i j}^{1}\right) \circ G\left(g_{i}\right) a\right\}_{i j} \rightarrow\left\{G\left(g_{i} \circ \pi_{i j}^{1}\right) a\right\}_{i j}$ for all objects $a$ of $G(B)$. For the identity morphisms $1_{X}, 1_{Y}$, and $1_{Z}$ of $\mathcal{J}$ we define $\tau_{1_{X}}^{\prime}, \tau_{1_{Y}}^{\prime}$ and $\tau_{1_{Z}}^{\prime}$ to be equalities. The coherence diagram for the unit axiom of pseudo natural transformations is satisfied because of this definition. The composition axiom for $\tau^{\prime}$ and nontrivial morphisms is satisfied because of the composition axiom for $\gamma^{G}$ and because $\gamma^{\Delta_{G(B)}}$ is an equality. The composition axiom for $\tau^{\prime}$ whenever one or more of the morphisms is trivial follows trivially. Thus $\pi^{\prime}: \Delta_{G(B)} \Rightarrow F$ is a pseudo natural transformation with coherence isos $\tau^{\prime}$. After these preliminary remarks, we can finally define stack of categories.

Definition 11.3. Let $C$ at denote the 2-category of small categories. Suppose $\mathcal{B}$ is a Grothendieck topology. A stack of categories is a contravariant pseudo functor $G: \mathcal{B} \rightarrow C$ at which takes Grothendieck covers to bilimits, i.e. for any object $B$ of $\mathcal{B}$ and any Grothendieck cover $\left\{g_{i}: B_{i} \rightarrow B \mid i \in I\right\} \in K(B)$ the diagram

$$
\prod_{i \in I} G\left(B_{i}\right) \underset{p_{2}}{\stackrel{p_{1}}{\rightleftarrows}} \prod_{i, j \in I} G\left(B_{i} \times_{B} B_{j}\right) \underset{p_{23}}{\stackrel{p_{12}}{\rightleftarrows}} \prod_{i, j, k \in I} G\left(B_{i} \times_{B} B_{j} \times_{B} B_{k}\right)
$$

has $G(B)$ as a bilimit with universal pseudo cone $\pi^{\prime}: \Delta_{G(B)} \Rightarrow F$ as defined above.

One common way to define a stack is via descent objects as in [15], 17, 40, or [52].

Definition 11.4. Let $\mathcal{B}$ be a Grothendieck topology and $G: \mathcal{B} \rightarrow$ Cat a contravariant pseudo functor. Suppose that $\left\{B_{i} \rightarrow B\right\}_{i}$ is a Grothendieck cover. Then an object with descent data on $\left\{B_{i} \rightarrow B\right\}_{i}$ consists of an object $\left\{a_{i}\right\}_{i} \in \prod_{i \in I} G\left(B_{i}\right)$ and isomorphisms $\phi_{i j}: G\left(\pi_{i j}^{2}\right) a_{j} \rightarrow G\left(\pi_{i j}^{1}\right) a_{i}$ in $G\left(B_{i} \times{ }_{B} B_{j}\right)$ which satisfy the cocycle condition

$$
G\left(\pi_{i j k}^{13}\right) \phi_{i k}=G\left(\pi_{i j k}^{12}\right) \phi_{i j} \circ G\left(\pi_{i j k}^{23}\right) \phi_{j k}
$$

in $G\left(B_{i} \times{ }_{B} B_{j} \times{ }_{B} B_{k}\right)$ up to the coherence isos of the pseudo functor $G$. See below. A morphism of descent objects $\left\{\xi_{i}\right\}_{i}:\left\{a_{i}\right\}_{i} \rightarrow\left\{a_{i}^{\prime}\right\}_{i}$ is a morphism in $\prod_{i \in I} G\left(B_{i}\right)$ 
such that the diagram

$$
\begin{gathered}
G\left(\pi_{i j}^{2}\right) a_{j} \stackrel{\phi_{i j}}{\longrightarrow} G\left(\pi_{i j}^{1}\right) a_{i} \\
G\left(\pi_{i j}^{2}\right) \xi_{j} \downarrow \\
G\left(\pi_{i j}^{2}\right) a_{j}^{\prime} \underset{\phi_{i j}^{\prime}}{\longrightarrow} G\left(\pi_{i j}^{1}\right) \xi_{i} \\
\longrightarrow
\end{gathered}
$$

commutes in $G\left(B_{i} \times_{B} B_{j}\right)$. These objects and morphisms form the category of descent data on the cover $\left\{B_{i} \rightarrow B\right\}_{i}$. This category is denoted $G\left(\left\{B_{i} \rightarrow B\right\}_{i}\right)$. There is a functor $G(B) \rightarrow G\left(\left\{B_{i} \rightarrow B\right\}_{i}\right)$ defined by $a \mapsto\left\{G\left(g_{i}\right) a\right\}_{i}$ where $g_{i}$ : $B_{i} \rightarrow B$ are the morphisms from the Grothendieck cover. The $\phi_{i j}$ belonging to the image of $a$ under this functor are $\phi_{i j}:=\left(\gamma_{\pi_{i j}^{1}, g_{i}}^{G} a\right)^{-1} \circ\left(\gamma_{\pi_{i j}^{2}, g_{j}}^{G} a\right)$.

The cocycle condition can be stated explicitly as the requirement that the following diagram commutes.

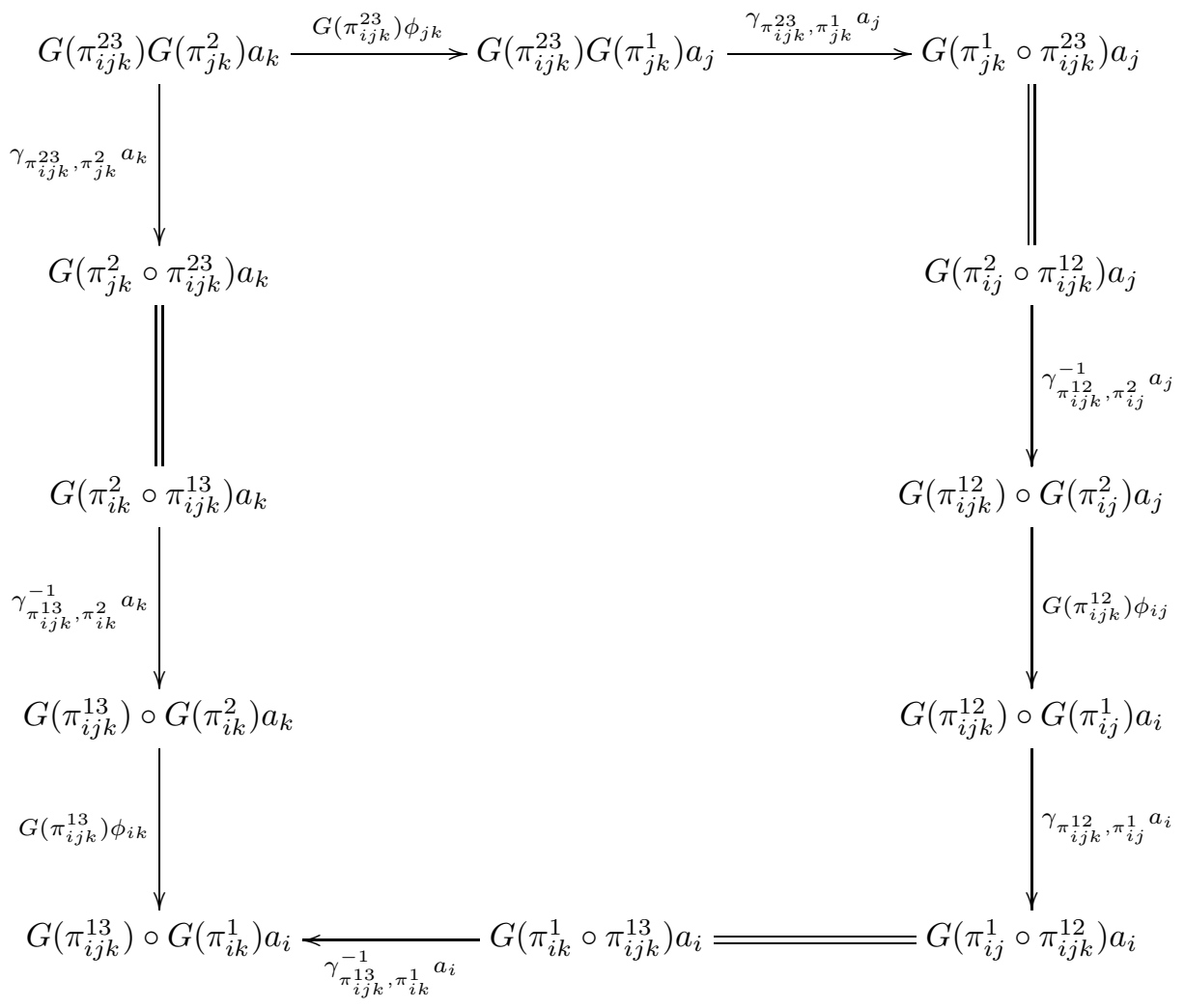

This diagram is another reason why we require our pseudo functors to have coherence arrows that are iso: if $\gamma$ were not invertible, the cocycle condition cannot be stated.

Definition 11.5. Let $C$ at denote the 2-category of small categories. Suppose $\mathcal{B}$ is a Grothendieck topology. A Giraud stack of categories $^{1}$ is a contravariant pseudo

\footnotetext{
${ }^{1}$ This is not standard terminology. We have only introduced it to show that the two definitions are equivalent.
} 
functor $G: \mathcal{B} \rightarrow C$ at such that for any object $B$ of $\mathcal{B}$ and any Grothendieck cover $\left\{B_{i} \rightarrow B\right\}_{i}$ of $B$, the functor $G(B) \rightarrow G\left(\left\{B_{i} \rightarrow B\right\}_{i}\right)$ is an equivalence of categories.

Theorem 11.1. Let $G: \mathcal{B} \rightarrow$ Cat be a pseudo functor from a Grothendieck topology to the 2-category of small categories. Then $G$ is a stack if and only if it is a Giraud stack.

Proof:

From Section 4 we know that the category $L:=P \operatorname{seudoCone}(\mathbf{1}, F)$ is a pseudo limit of $F$. It is described as a subcategory of an appropriate product in Remarks 4.4 and 4.5 in such a way that the pseudo cone $\pi: \Delta_{L} \Rightarrow F$ consists of projections as in Remark 4.6]

I claim that the category $L$ of pseudo cones is equivalent to the category $G\left(\left\{B_{i} \rightarrow\right.\right.$ $\left.B\}_{i}\right)$ of descent data by a functor $H: L \rightarrow G\left(\left\{B_{i} \rightarrow B\right\}_{i}\right)$. Recall from Remark 4.4 that each object of $L$ corresponds to a tuple

$$
\left\{a_{i}\right\}_{i} \times\left\{a_{i j}\right\}_{i j} \times\left\{a_{i j k}\right\}_{i j k} \times\left\{\varepsilon_{f}\right\}_{f}
$$

with objects $\left\{a_{i}\right\}_{i}$ in $\prod_{i} G\left(B_{i}\right),\left\{a_{i j}\right\}_{i j}$ in $\prod_{i j} G\left(B_{i} \times_{B} B_{j}\right)$, and $\left\{a_{i j k}\right\}_{i j k}$ in $\prod_{i j k} G\left(B_{i} \times_{B} B_{j} \times_{B} B_{k}\right)$ as well as morphisms $\varepsilon_{f}$ indexed by morphisms $f$ of $\mathcal{J}$ appropriately. For example, $\varepsilon_{f_{1}}: F\left(f_{1}\right)\left\{a_{i}\right\}_{i} \rightarrow\left\{a_{i j}\right\}_{i j}$ These morphisms satisfy two axioms listed in the Remark. Each morphism of $L$ corresponds to a tuple

$$
\left\{\xi_{i}\right\}_{i} \times\left\{\xi_{i j}\right\}_{i j} \times\left\{\xi_{i j k}\right\}_{i j k}
$$

of morphisms in the product categories above and this tuple commutes with the morphisms $\varepsilon_{f}$ appropriately. Define

$$
\begin{gathered}
H\left(\left\{a_{i}\right\}_{i} \times\left\{a_{i j}\right\}_{i j} \times\left\{a_{i j k}\right\}_{i j k} \times\left\{\varepsilon_{f}\right\}_{f}\right):=\left\{a_{i}\right\}_{i} \\
H\left(\left\{\xi_{i}\right\}_{i} \times\left\{\xi_{i j}\right\}_{i j} \times\left\{\xi_{i j k}\right\}_{i j k}\right):=\left\{\xi_{i}\right\}_{i} .
\end{gathered}
$$

The descent data for $\left\{a_{i}\right\}_{i}$ are defined as the components of $\left\{\phi_{i j}\right\}_{i j}:=\left(\varepsilon_{f_{1}}\right)^{-1} \circ \varepsilon_{f_{2}}$. Morphisms of $L$ map to morphisms of $G\left(\left\{B_{i} \rightarrow B\right\}_{i}\right)$ because the outer diagram of

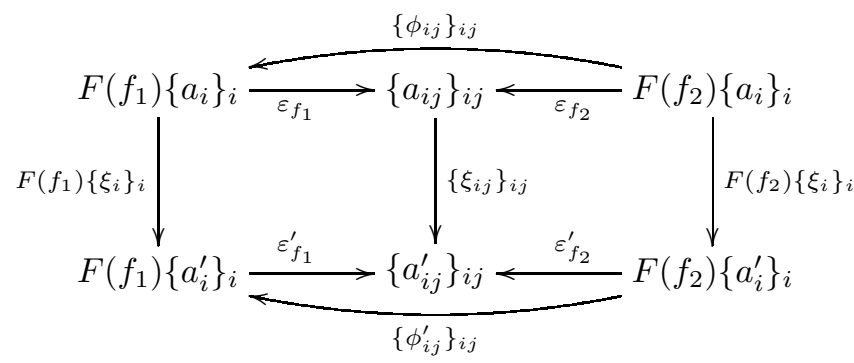


commutes by Remark 4.5 To see that the $\phi_{i j}$ satisfy the cocycle condition, consider the diagram below.

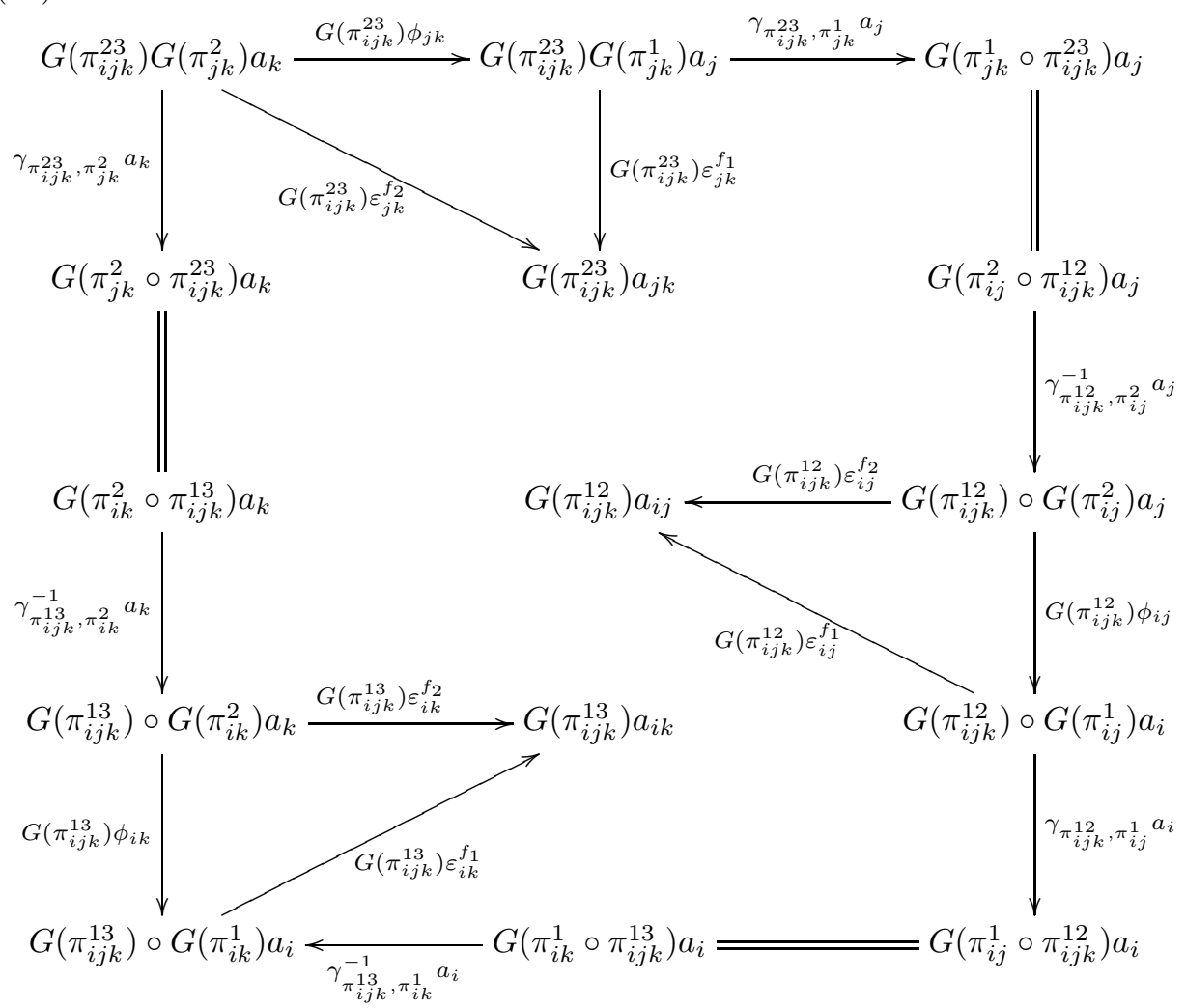

We want to show that the outer rectangle commutes. The small triangles commute by definition of $\phi_{i j}$. Next one draws another vertex $a_{i j k}$ inside the rectangle but outside the triangles. Then one draws the arrows $\varepsilon_{i j k}^{f}$ for all non-identity morphisms $F$ of the category $\mathcal{J}$ with target $Z$. All of these arrows terminate at $a_{i j k}$. Each of the resulting subdiagrams commutes because of the relations in $\mathcal{J}$ or because of the second axiom on the morphisms $\varepsilon_{f}$ in Remark 4.4. Note that we are using the notation $\varepsilon_{f}=\left\{\varepsilon_{i j k}^{f}\right\}_{i j k}$. The outer rectangle commute because all of the subdiagrams commute and everything is iso. Hence the $\phi_{i j}$ 's satisfy the cocycle condition and $H$ maps $L$ into $G\left(\left\{B_{i} \rightarrow B\right\}_{i}\right)$. These assignments obviously define a functor $H$.

The functor $H$ is faithful. Suppose

$$
H\left(\left\{\xi_{i}\right\}_{i} \times\left\{\xi_{i j}\right\}_{i j} \times\left\{\xi_{i j k}\right\}_{i j k}\right)=H\left(\left\{\xi_{i}^{\prime}\right\}_{i} \times\left\{\xi_{i j}^{\prime}\right\}_{i j} \times\left\{\xi_{i j k}^{\prime}\right\}_{i j k}\right) .
$$

Then $\left\{\xi_{i}\right\}_{i}=\left\{\xi_{i}^{\prime}\right\}_{i}$. From this we conclude $\left\{\xi_{i j}\right\}_{i j}=\left\{\xi_{i j}^{\prime}\right\}_{i j}$ by diagram (58). A similar diagram with objects $\left\{a_{i j k}\right\}$ and $\left\{a_{i j k}^{\prime}\right\}$ in the center and arrows $\varepsilon_{f_{12}}, \varepsilon_{f_{23}}$ and $\varepsilon_{f_{12}}^{\prime}, \varepsilon_{f_{23}}^{\prime}$ pointing inward shows that $\left\{\xi_{i j k}\right\}_{i j k}=\left\{\xi_{i j k}^{\prime}\right\}_{i j k}$.

The functor $H$ is also full. Let $\left\{\xi_{i}\right\}_{i}$ be a morphism in the category of descent data. Suppose further that its source and target lie in the image of $H$. Then the outer diagram of diagram (58) commutes and we define $\left\{\xi_{i j}\right\}_{i j}$ to be the unique arrow that makes diagram (58) commute. It exists because the horizontal arrows 
are iso. One can also define $\left\{\xi_{i j k}\right\}_{i j k}$ similarly, although one needs to use diagram (58) several times and the naturality of $\gamma^{G}$ to show that the necessary diagrams in Remark 4.5 commute.

The functor $H$ is also surjective on objects. Suppose $\left\{a_{i}\right\}$ is an object with descent data $\phi_{i j}$. Define $a_{i j}:=G\left(\pi_{i j}^{1}\right) a_{i}$ and $a_{i j k}:=G\left(\pi_{i k}^{1} \circ \pi_{i j k}^{13}\right) a_{i}$. Define $\varepsilon_{i j}^{f_{1}}$ : $G\left(\pi_{i j}^{1}\right) a_{i} \rightarrow a_{i j}$ to be the identity and $\varepsilon_{i j}^{f_{2}}:=\phi_{i j}$. Let $\varepsilon_{i j k}^{f_{13} \circ f_{1}}: G\left(\pi_{i k}^{1} \circ \pi_{i j k}^{13}\right) a_{i} \rightarrow a_{i j k}$ also be the identity. Any $\varepsilon$ indexed by an identity morphism is also trivial. Consider diagram (59) with the additional vertex $a_{i j k}$ and the additional $\varepsilon$ 's mentioned just after diagram (59). Requiring the inner diagrams to commute uniquely defines the other $\varepsilon$ 's which we did not define yet. The commutivity of these smaller diagrams guarantees that the tuple

$$
\left\{a_{i}\right\}_{i} \times\left\{a_{i j}\right\}_{i j} \times\left\{a_{i j k}\right\}_{i j k} \times\left\{\varepsilon_{f}\right\}_{f}
$$

we have just defined is an object of $L$. This object obviously maps under $H$ to $\left\{a_{i}\right\}_{i}$ with the correct descent data.

We conclude $H$ is an equivalence because it is faithfully full and essentially surjective. Hence the category $L$ of pseudo cones is equivalent to the category $G\left(\left\{B_{i} \rightarrow B\right\}_{i}\right)$ of descent data.

There is also a functor $G(B) \rightarrow L$ defined similarly to the functor $G(B) \rightarrow$ $G\left(\left\{B_{i} \rightarrow B\right\}_{i}\right)$ that makes the diagrams

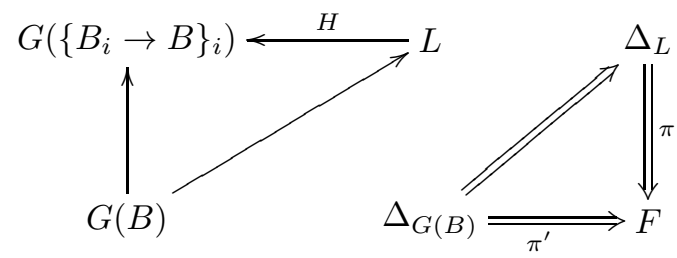

commute. Suppose $G$ is a Giraud stack. Then the left vertical arrow is an equivalence. Hence the functor $G(B) \rightarrow L$ is an equivalence and $\pi^{\prime}$ makes $G(B)$ into a bilimit of $F$ because $L$ is a bilimit of $F$ with pseudo limiting cone $\pi$. Hence $G$ is a stack.

Suppose $G$ is a stack. Then $\pi^{\prime}$ makes $G(B)$ into a bilimit of $F$. Then the functor $G(B) \rightarrow L$ is an equivalence because $L$ is also a bilimit and the right diagram commutes. Hence the functor $G(B) \rightarrow G\left(\left\{B_{i} \rightarrow B\right\}_{i}\right)$ is also an equivalence and $G$ is a Giraud stack.

This completes the proof that the two definitions of stack are equivalent.

Next we define stacks of objects in a 2-category which admits bilimits, such as the 2-category of pseudo algebras over a theory.

Definition 11.6. Let $\mathcal{C}$ be a 2-category whose objects have underlying categories. Suppose $\mathcal{B}$ is a Grothendieck topology and $\mathcal{C}$ admits bilimits. A stack of objects of $\mathcal{C}$ is a contravariant pseudo functor $G: \mathcal{B} \rightarrow \mathcal{C}$ which takes Grothendieck covers to bilimits, i.e. for any object $B$ of $\mathcal{B}$ and any Grothendieck cover $\left\{g_{i}: B_{i} \rightarrow B \mid i \in\right.$ $I\} \in K(B)$ the diagram

$$
\prod_{i \in I} G\left(B_{i}\right) \underset{p_{2}}{\stackrel{p_{1}}{\rightleftarrows}} \prod_{i, j \in I} G\left(B_{i} \times_{B} B_{j}\right) \underset{p_{23}}{\stackrel{p_{12}}{\rightleftharpoons}} \prod_{i, j, k \in I} G\left(B_{i} \times_{B} B_{j} \times_{B} B_{k}\right)
$$


has $G(B)$ as a bilimit with universal pseudo cone $\pi^{\prime}: \Delta_{G(B)} \Rightarrow F$ as defined above.

For example, a stack of pseudo algebras over a theory $T$ is a contravariant pseudo functor from a Grothendieck topology into the 2-category of pseudo $T$-algebas which takes Grothendieck covers to bilimits in the above sense.

\section{2-Theories, Algebras, and Weighted Pseudo Limits}

The algebraic structure of the category of rigged surfaces can be described as a pseudo algebra over a certain 2-theory (see [24, 25], and 26], where however the term lax algebra over a 2-theory is used for what we call a pseudo algebra over a 2-theory). In this section we review the relevant terminology and prove results about limits. Before giving the definition of a 2-theory, we motivate it with an example in the following subsection.

12.1. The 2-Theory End(X) Fibered over the Theory End(I). Let $I$ be a category and $k$ a positive integer. Suppose $X: I^{k} \rightarrow C a t$ is a strict 2 -functor from the category $I^{k}$ to the 2-category Cat of small categories. Here $I^{k}$ is interpreted as a 2-category where the Hom sets are discrete categories. We will now describe the 2-theory $\operatorname{End}(X)$ fibered over the theory End(I), which is a contravariant functor $\operatorname{End}(I) \rightarrow$ Cat satisfying certain properties.

Recall that the theory $\operatorname{End}(I)$ is the category with objects $0=\{*\}, 1=I, 2=$ $I^{2}, 3=\ldots$ and morphisms $\operatorname{Mor}_{\operatorname{End}(I)}(m, n)=$ Functors $\left(I^{m}, I^{n}\right)$. Here $\{*\}$ denotes the terminal object in the category of small categories. As with any theory, the theory $\operatorname{End}(I)$ can be completely described by the sets $\operatorname{End}(I)(n):=\operatorname{Mor}_{\operatorname{End}(I)}(n, 1)$ and a list of axioms. See the section on theories or 24 for details.

From the theory $\operatorname{End}(I)$ we can obtain another category denoted $\operatorname{End}(I)^{k}$, which also turns out to be a theory. It has objects $0=\{*\} \times \cdots \times\{*\}, 1=I \times \cdots \times$ $I, 2=I^{2} \times \cdots \times I^{2}, 3=\ldots$ ( $k$ copies in each product $)$ and it has morphisms $\operatorname{Mor}_{\operatorname{End}(I)^{k}}(m, n):=\operatorname{Mor}_{\operatorname{End}(I)}(m, n)^{\times k}$. For example, $v \in \operatorname{Mor}_{\operatorname{End}(I)^{k}}(m, 1)$ is a functor $v:\left(I^{m}\right)^{k} \rightarrow I^{k}$ that is a $k$-tuple of functors $I^{m} \rightarrow I$. For $n \in \mathbb{N}$ and $1 \leq i \leq n$ let $p r_{i}^{\times k}:\left(I^{n}\right)^{k} \rightarrow I^{k}$ be the morphism $p r_{i}^{\times k} \in \operatorname{Mor}_{\operatorname{End}(I)^{k}}(n, 1)$ whose $k$ components are each the projection functor $\mathrm{pr}_{i}: I^{n} \rightarrow I$ onto the $i$-th coordinate. One can easily check that $n \in \operatorname{Obj} \operatorname{End}(I)^{k}$ is the product in $\operatorname{End}(I)^{k}$ of $n$ copies of 1 with projection morphisms $p r_{1}^{\times k}, \ldots, p r_{n}^{\times k}$. Hence $\operatorname{End}(I)^{k}$ is itself a theory and $\operatorname{Mor}_{\operatorname{End}(I)^{k}}(m, n)$ is in bijective correspondence with $\prod_{i=1}^{n} \operatorname{Mor}_{\operatorname{End}(I)^{k}}(m, 1)$. We identify these two sets via the usual bijection. In other words, for $k$-tuples $w_{1}, \ldots, w_{n} \in \operatorname{Mor}_{\operatorname{End}(I)^{k}}(m, 1)$ we let $\prod_{j=1}^{n} w_{j}$ denote the unique morphism $m \rightarrow n$ of $\operatorname{End}(I)^{k}$ such that

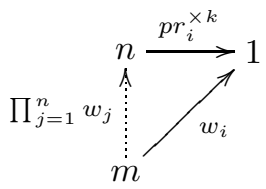

commutes for all $i=1, \ldots, n .^{2}$ Using this convention, we have $w=\prod_{j=1}^{n} p r_{j}^{\times k} \circ w$ for $w \in \operatorname{Mor}_{\operatorname{End}(I)^{k}}(m, n)$.

\footnotetext{
${ }^{2}$ This notation differs from 24 . In 24 the notation $\left(w_{1}, \ldots, w_{n}\right)$ is used instead of the product. I reserve $\left(w_{1}, \ldots, w_{n}\right)$ for something else. The reason for my choice will become clear later.
} 
Since $\operatorname{End}(I)^{k}$ is a theory, it has a substitution and a composition with unit which satisfy certain axioms described in 24] and the section on theories in this paper. If $f:\{1, \ldots, p\} \rightarrow\{1, \ldots, q\}$ is a function and $w \in \operatorname{End}(I)^{k}(p)=\operatorname{Mor}_{\operatorname{End}(I)^{k}}(p, 1)=$ $\operatorname{Mor}_{\operatorname{End}(I)}(p, 1)^{\times k}$, then the substituted word $w_{f}$ is obtained by substituting by $f$ in each of the words in the $k$-components of $w$. The composition is also done componentwise. The unit $1^{\times k}: I \times \cdots \times I \rightarrow I \times \cdots \times I$ is $k$ copies of the unit $1: I \rightarrow I$ in the theory $\operatorname{End}(I)$. These explicit descriptions of substitution, composition, and unit follow from the definitions of the projections in the theory $\operatorname{End}(I)^{k}$ by the work in the section on theories.

We follow the convention introduced earlier to define a morphism $\left(w_{1}, \ldots, w_{n}\right)$. Let $w_{i} \in \operatorname{End}(I)^{k}\left(m_{i}\right)$ for $i=1, \ldots, n$. Let $\iota_{i}:\left\{1, \ldots, m_{i}\right\} \rightarrow\left\{1, \ldots, m_{1}+m_{2}+\right.$ $\left.\cdots+m_{n}\right\}$ be the injective function which takes the domain to the $i$-th block as in the section on theories. Then there exists a unique morphism $\left(w_{1}, \ldots, w_{n}\right)$ such that

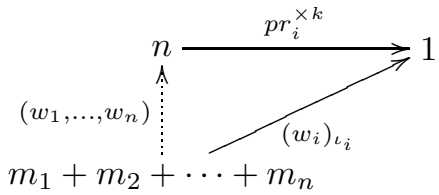

commutes for all $i=1, \ldots, n$. Explicitly, the morphism $\left(w_{1}, \ldots, w_{n}\right)$ is obtained by doing an analogous process in each of the $k$ components.

The strict 2-functor $X: I^{k} \rightarrow C$ at gives rise to a contravariant functor $\operatorname{End}(X)$ : $\operatorname{End}(I) \rightarrow C a t$ as follows. For $m \in \operatorname{Obj} \operatorname{End}(I)$ the category $\operatorname{End}(X)(m)$ has objects $\operatorname{Obj} \operatorname{End}(X)(m)=\coprod_{n \geq 0} \operatorname{Mor}_{E n d(I)^{k}}(m, n)$. For $\prod_{i=1}^{p} v_{i}, \prod_{i=1}^{q} w_{i} \in \operatorname{ObjEnd}(X)(m)$ where $v_{1}, \ldots, v_{p}, w_{1}, \ldots, w_{q} \in \operatorname{Mor}_{\operatorname{End}(I)^{k}}(m, 1)$ we define $\operatorname{Mor}_{\operatorname{End}(X)(m)}\left(\prod_{i=1}^{p} v_{i}, \prod_{i=1}^{q} w_{i}\right)$ to be the collection of natural transformations

$$
\alpha: X \circ v_{1} \circ d^{m} \times \cdots \times X \circ v_{p} \circ d^{m} \Rightarrow X \circ w_{1} \circ d^{m} \times \cdots \times X \circ w_{q} \circ d^{m}
$$

where $d^{m}: I^{m} \rightarrow\left(I^{m}\right)^{k}$ is the diagonal functor. Note that $X \circ v_{1} \circ d^{m} \times \cdots \times$ $X \circ v_{p} \circ d^{m}$ and $X \circ w_{1} \circ d^{m} \times \cdots \times X \circ w_{q} \circ d^{m}$ are functors $I^{m} \rightarrow$ Cat. The composition of morphisms in $\operatorname{End}(X)(m)$ is the vertical composition of natural transformations. With these definitions, $\operatorname{End}(X)(m)$ is a category. We must still define the contravariant functor $\operatorname{End}(X)$ on morphisms and verify that it preserves identities and compositions. For any morphism $u: I^{\ell} \rightarrow I^{m}$ of the theory $\operatorname{End}(I)$, define $u^{\times k}:\left(I^{\ell}\right)^{k} \rightarrow\left(I^{m}\right)^{k}$ to be the functor which is $u$ in each of the $k$ components. Note that $u^{\times k} \circ d^{\ell}=d^{m} \circ u: I^{\ell} \rightarrow\left(I^{m}\right)^{k}$. The functor $\operatorname{End}(X)(u): \operatorname{End}(X)(m) \rightarrow$ $\operatorname{End}(X)(\ell)$ is defined on objects by $\operatorname{End}(X)(u)\left(\prod_{i=1}^{p} v_{i}\right):=\prod_{i=1}^{p} v_{i} \circ u^{\times k}$ and on morphisms $\alpha$ in (60) by $\operatorname{End}(X)(u)(\alpha):=\alpha * i_{u}$ where $*$ denotes the horizontal composition of natural transformations and $i_{u}: u \Rightarrow u$ is the trivial natural transformation. This makes sense because

$$
\begin{aligned}
\left(X \circ v_{1} \circ d^{m} \times \cdots \times X \circ v_{p} \circ d^{m}\right) \circ u & =X \circ v_{1} \circ d^{m} \circ u \times \cdots \times X \circ v_{p} \circ d^{m} \circ u \\
& =X \circ v_{1} \circ u^{\times k} \circ d^{\ell} \times \cdots \times X \circ v_{p} \circ u^{\times k} \circ d^{\ell}
\end{aligned}
$$

and

$$
\alpha * i_{u}: X \circ v_{1} \circ u^{\times k} \circ d^{\ell} \times \cdots \times X \circ v_{p} \circ u^{\times k} \circ d^{\ell} \Rightarrow X \circ w_{1} \circ u^{\times k} \circ d^{\ell} \times \cdots \times X \circ w_{q} \circ u^{\times k} \circ d^{\ell}
$$


really is a morphism

$$
\operatorname{End}(X)(u)\left(\prod_{i=1}^{p} v_{i}\right)=\prod_{i=1}^{p} v_{i} \circ u^{\times k} \rightarrow \prod_{i=1}^{p} w_{i} \circ u^{\times k}=\operatorname{End}(X)(u)\left(\prod_{i=1}^{q} w_{i}\right) .
$$

If $u: I^{\ell} \rightarrow I^{m}$ is the identity functor $I^{m} \rightarrow I^{m}$, then $\operatorname{End}(X)(u): \operatorname{End}(X)(m) \rightarrow$ $\operatorname{End}(X)(m)$ is also the identity functor because $v_{i} \circ u^{\times k}=v_{i}$ for $i=1, \ldots, p$ and $w_{i} \circ u^{\times k}=w_{i}$ for $i=1, \ldots, q$ and also $\alpha * i_{u}=\alpha$. If $I^{j} \stackrel{u_{1}}{\longrightarrow} I^{m} \stackrel{u_{2}}{\longrightarrow} I^{\ell}$ are morphisms in $\operatorname{End}(I)$, then $u_{2}^{\times k} \circ u_{1}^{\times k}=\left(u_{2} \circ u_{1}\right)^{\times k}$ and

$$
\left(\alpha * i_{u_{2}}\right) * i_{u_{1}}=\alpha *\left(i_{u_{2}} * i_{u_{1}}\right)=\alpha * i_{u_{2} \circ u_{1}},
$$

which together imply that

$$
\operatorname{End}(X)\left(u_{2} \circ u_{1}\right)=\operatorname{End}(X)\left(u_{1}\right) \circ \operatorname{End}(X)\left(u_{2}\right) .
$$

Thus $\operatorname{End}(X): \operatorname{End}(I) \rightarrow$ Cat preserves identities and compositions and is a contravariant functor.

The category $\operatorname{End}(X)(m)$ also has certain products, which will be a feature of a general 2-theory. For $v_{1}, \ldots, v_{p} \in \operatorname{Mor}_{\operatorname{End}(I)^{k}}(m, 1)$ and $\prod_{i=1}^{p} v_{i} \in \operatorname{Mor}_{\operatorname{End}(I)^{k}}(m, p) \subseteq$ $\operatorname{Obj} \operatorname{End}(X)(m)$ define projections $p r_{j}: \prod_{i=1}^{p} v_{i} \rightarrow v_{j}$ for $j=1, \ldots, p$ to be the projection natural transformations

$$
X \circ v_{1} \circ d^{m} \times \cdots \times X \circ v_{p} \circ d^{m} \Rightarrow X \circ v_{j} \circ d^{m} .
$$

Then $\prod_{i=1}^{p} v_{i}$ is obviously the product of $v_{1}, \ldots, v_{p}$ in the category $\operatorname{End}(X)(m)$ with these projections. This explains the choice of notation. This product property will also be required of a general 2-theory. We record for later use how these products allow us to define morphisms $\iota^{\prime}$ for every function $\iota:\{1, \ldots, p\} \rightarrow\{1, \ldots, q\}$. Let $w_{1}, \ldots, w_{q} \in \operatorname{Mor}_{\operatorname{End}(I)^{k}}(m, 1) \subseteq \operatorname{ObjEnd}(X)(m)$. Then for a function $\iota$ : $\{1, \ldots, p\} \rightarrow\{1, \ldots, q\}$ there exists a unique morphism $\iota^{\prime}$ such that

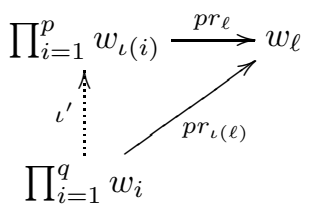

commutes for all $\ell=1, \ldots, p$. The arrows of the natural transformation $\iota^{\prime}: X \circ w_{1} \circ$ $d^{m} \times \cdots \times X \circ w_{q} \circ d^{m} \Rightarrow X \circ w_{\iota(1)} \circ d^{m} \times \cdots \times X \circ w_{\iota(p)} \circ d^{m}$ have the appropriate projections as their components.

The 2-theory $\operatorname{End}(X)$ has several operations on it which any general 2-theory will also have, once we define the notion of 2-theory. To make the description of these operations easier, we follow the notation introduced by P. Hu and I. Kriz in 24]. For objects $w_{1}, \ldots, w_{q}, w \in \operatorname{Mor}_{\operatorname{End}(I)^{k}}(m, 1) \subseteq \operatorname{ObjEnd}(X)(m)$ we set

$$
\operatorname{End}(X)\left(w ; w_{1}, \ldots, w_{q}\right):=\operatorname{Mor}_{\operatorname{End}(X)(m)}\left(\prod_{i=1}^{q} w_{i}, w\right) .
$$

The operations of P. Hu and I. Kriz are collated in the following theorem.

Theorem 12.1. The contravariant functor $\operatorname{End}(X): \operatorname{End}(I) \rightarrow$ Cat has the following operations.

(1) For each $w \in T^{k}(m)$ there exists a unit $1_{w} \in \operatorname{End}(X)(w ; w)$. 
(2) For all $w, w_{i}, w_{i j} \in \operatorname{Mor}_{\operatorname{End}(I)^{k}}(m, 1)$ there is a function

$$
\begin{aligned}
& \gamma: \operatorname{End}(X)\left(w ; w_{1}, \ldots, w_{q}\right) \times \operatorname{End}(X)\left(w_{1} ; w_{11}, \ldots, w_{1 p_{1}}\right) \times \cdots \times \operatorname{End}(X)\left(w_{q} ; w_{q 1}, \ldots, w_{q p_{q}}\right) \\
& \rightarrow \operatorname{End}(X)\left(w ; w_{11}, \ldots, w_{q p_{q}}\right)
\end{aligned}
$$

called $\operatorname{End}(X)$-composition.

(3) Let $w, w_{1}, \ldots, w_{q} \in M_{\text {or }}{\operatorname{End}(I)^{k}}(m, 1)$. For any function $\iota:\{1, \ldots, p\} \rightarrow$ $\{1, \ldots, q\}$ there is a function

$$
()^{\iota}: \operatorname{End}(X)\left(w ; w_{\iota(1)}, \ldots, w_{\iota(p)}\right) \rightarrow \operatorname{End}(X)\left(w ; w_{1}, \ldots, w_{q}\right)
$$

called End $(X)$-functoriality.

(4) Let $w, w_{1}, \ldots, w_{q} \in \operatorname{Mor}_{\operatorname{End}(I)^{k}}(m, 1)$. For any function $f:\{1, \ldots, m\} \rightarrow$ $\{1, \ldots, \ell\}$ there is a function

$$
()_{f}: \operatorname{End}(X)\left(w ; w_{1}, \ldots, w_{q}\right) \rightarrow \operatorname{End}(X)\left(w_{f} ;\left(w_{1}\right)_{f}, \ldots,\left(w_{q}\right)_{f}\right)
$$

where $w_{f}$ means to substitute $f$ in each of the words in the $k$-tuple $w$. This function is called End $(I)$-functoriality. Note that $\operatorname{End}(X)\left(w ; w_{1}, \ldots, w_{q}\right)$ is a Hom set in the category $\operatorname{End}(X)(m)$ while $\operatorname{End}(X)\left(w_{f} ;\left(w_{1}\right)_{f}, \ldots,\left(w_{q}\right)_{f}\right)$ is a Hom set in the category $\operatorname{End}(X)(\ell)$.

(5) For $u_{i} \in \operatorname{End}(I)\left(k_{i}\right), i=1, \ldots, m$, and $v_{i}:=\gamma^{\times k}\left(w_{i} ; u_{1}^{\times k}, \ldots, u_{m}^{\times k}\right)$ and $v:=\gamma^{\times k}\left(w ; u_{1}^{\times k}, \ldots, u_{m}^{\times k}\right)$ there is a function

$$
\left(u_{1}, \ldots, u_{m}\right)^{*}: \operatorname{End}(X)\left(w ; w_{1}, \ldots, w_{q}\right) \rightarrow \operatorname{End}(X)\left(v ; v_{1}, \ldots, v_{q}\right) .
$$

This function is called End(I)-substitution. Here $\gamma^{\times k}$ means to use the composition of the theory End(I) in each of the $k$ components, which coincides with composition in the theory $\operatorname{End}(I)^{k}$. Note that $\operatorname{End}(X)\left(w ; w_{1}, \ldots, w_{q}\right)$ is a Hom set in the category $\operatorname{End}(X)(m)$ while $\operatorname{End}(X)\left(v ; v_{1}, \ldots, v_{q}\right)$ is a Hom set in the category $\operatorname{End}(X)\left(k_{1}+\cdots+k_{m}\right)$.

Proof:

(1) The unit $1_{w}: X \circ w \circ d^{m} \Rightarrow X \circ w \circ d^{m}$ is the identity natural transformation $i_{X \circ w \circ d^{m}}: X \circ w \circ d^{m} \Rightarrow X \circ w \circ d^{m}$.

(2) Let $\alpha: \prod_{i=1}^{q} w_{i} \rightarrow w$ and $\alpha_{i}: \prod_{j=1}^{p_{i}} w_{i j} \rightarrow w_{i}$ for $i=1, \ldots, q$ be morphisms. Let $\iota_{\ell}:\left\{1, \ldots, p_{\ell}\right\} \rightarrow\left\{1, \ldots, p_{1}+p_{2}+\cdots+p_{q}\right\}$ be the injective function which takes the domain to the $\ell$-th block. We take the product $\prod_{i=1}^{q} \prod_{j=1}^{p_{i}} w_{i j}$ to be

$$
\prod_{i=1}^{q} \prod_{j=1}^{p_{i}} w_{i j}=w_{11} \times w_{12} \times \cdots w_{1 p_{1}} \times w_{21} \times \cdots \times w_{2 p_{2}} \times w_{31} \times \cdots \times w_{q p_{q}} .
$$

Then there exists a unique morphism $\left(\alpha_{1}, \ldots, \alpha_{q}\right)$ such that

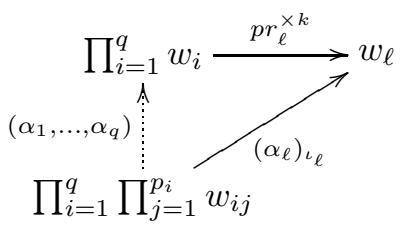

commutes for all $\ell=1, \ldots, q$. This means that

$$
\left(\alpha_{1}, \ldots, \alpha_{q}\right): X \circ w_{11} \circ d^{m} \times \cdots \times X \circ w_{q p_{q}} \circ d^{m} \Rightarrow X \circ w_{1} \circ d^{m} \times X \circ w_{2} \circ d^{m} \times \cdots \times X \circ w_{q} \circ d^{m}
$$


is the natural transformation which is $\alpha_{\ell}$ on $X \circ w_{\ell 1} \circ d^{m} \times \cdots \times X \circ w_{\ell p_{\ell}} \circ d^{m}$. Define

$$
\gamma\left(\alpha ; \alpha_{1}, \ldots, \alpha_{q}\right):=\alpha \circ\left(\alpha_{1}, \ldots \alpha_{q}\right)
$$

where the composition is in the category $\operatorname{End}(X)(m)$.

(3) Let $w_{1}, \ldots, w_{q} \in \operatorname{Mor}_{\operatorname{End}(I)^{k}}(m, 1)$ and $\iota:\{1, \ldots, p\} \rightarrow\{1, \ldots, q\}$ be a function. Let $\iota^{\prime}: \prod_{i=1}^{q} w_{i} \rightarrow \prod_{i=1}^{q} w_{\iota(i)}$ be the morphism defined in diagram (61). Then define $\operatorname{End}(X)$-functoriality $\operatorname{End}(X)\left(w ; w_{\iota(1)}, \ldots, w_{\iota(p)}\right) \rightarrow \operatorname{End}(X)\left(w ; w_{1}, \ldots, w_{q}\right)$ by $\alpha \mapsto \alpha \circ \iota^{\prime}$.

(4) A function $f:\{1, \ldots, m\} \rightarrow\{1, \ldots, \ell\}$ induces a morphism $f^{\prime}: \ell \rightarrow m$ in $\operatorname{End}(I)$ which in turn gives rise to a morphism $\left(f^{\prime}\right)^{\times k}:\left(I^{\ell}\right)^{k} \rightarrow\left(I^{m}\right)^{k}$ in $\operatorname{End}(I)^{k}$. Then $w_{f}=w \circ\left(f^{\prime}\right)^{\times k}$ by definition and the functor $\operatorname{End}(X)\left(f^{\prime}\right)$ : $\operatorname{End}(X)(m) \rightarrow \operatorname{End}(X)(\ell)$ gives us a map of Hom sets

$$
()_{f}: \operatorname{End}(X)\left(w ; w_{1}, \ldots, w_{q}\right) \rightarrow \operatorname{End}(X)\left(w_{f} ;\left(w_{1}\right)_{f}, \ldots,\left(w_{q}\right)_{f}\right) .
$$

(5) Let $\iota_{i}:\left\{1, \ldots, k_{i}\right\} \rightarrow\left\{1, \ldots, k_{1}+k_{2}+\cdots+k_{m}\right\}$ be the injective map which takes the domain to the $i$-th block. Let $\left(u_{1}^{\times k}, \ldots, u_{m}^{\times k}\right)$ denote the unique morphism in $\operatorname{End}(I)^{k}$ such that

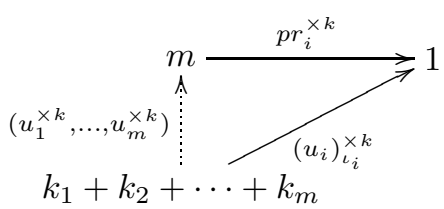

commutes. Then we know from the general theory of theories that $\gamma^{\times k}\left(w ; u_{1}^{\times k}, \ldots, u_{m}^{\times k}\right)=w \circ\left(u_{1}^{\times k}, \ldots, u_{m}^{\times k}\right)$ where the composition "o" is the composition in the category $\operatorname{End}(I)^{k}$. Then $\operatorname{End}(X)\left(u_{1}^{\times k}, \ldots, u_{m}^{\times k}\right)(w)=v$ and the functor $\operatorname{End}(X)\left(u_{1}^{\times k}, \ldots, u_{m}^{\times k}\right)$ gives us the desired map of Hom sets.

These operations on $\operatorname{End}(X)$ satisfy certain axioms.

Theorem 12.2. The operations on the contravariant functor $\operatorname{End}(X): \operatorname{End}(I) \rightarrow$ Cat satisfy the following relations.

(1) $\operatorname{End}(X)$-composition is associative, i.e. $\gamma\left(\alpha ; \gamma\left(\alpha^{1} ; \alpha_{1}^{1}, \ldots, \alpha_{n_{1}}^{1}\right), \gamma\left(\alpha^{2} ; \alpha_{1}^{2}, \ldots, \alpha_{n_{2}}^{2}\right), \ldots, \gamma\left(\alpha^{q} ; \alpha_{1}^{q}, \ldots, \alpha_{n_{q}}^{q}\right)\right)$ is the same as $\gamma\left(\gamma\left(\alpha ; \alpha^{1}, \ldots, \alpha^{q}\right), \alpha_{1}^{1}, \ldots \alpha_{n_{1}}^{1}, \alpha_{1}^{2}, \ldots, \alpha_{n_{2}}^{2}, \ldots, \alpha_{1}^{q}, \ldots, \alpha_{n_{q}}^{q}\right)$.

(2) $\operatorname{End}(X)$-composition is unital, i.e. for $\alpha \in \operatorname{End}(X)\left(w ; w_{1}, \ldots, w_{q}\right)$ we have $\gamma\left(\alpha ; 1_{w_{1}}, \ldots, 1_{w_{q}}\right)=\alpha=\gamma\left(1_{w} ; \alpha\right)$.

(3) $\operatorname{End}(X)$-functoriality is functorial, i.e.

for functions $\{1, \ldots, p\} \stackrel{\iota}{\longrightarrow}\{1, \ldots, q\} \stackrel{\theta}{\longrightarrow}\{1, \ldots, r\}$ the composition

$$
\operatorname{End}(X)\left(w ; w_{\theta \iota(1)}, \ldots, w_{\theta \iota(p)}\right) \stackrel{()^{\iota}}{\longrightarrow} \operatorname{End}(X)\left(w ; w_{\theta(1)}, \ldots, w_{\theta(q)}\right)
$$

$$
\stackrel{()^{\theta}}{\longrightarrow} \operatorname{End}(X)\left(w ; w_{1}, \ldots, w_{r}\right)
$$

is the same as

$$
\operatorname{End}(X)\left(w ; w_{\theta \iota(1)}, \ldots, w_{\theta \iota(p)}\right) \stackrel{()^{\theta \bullet \iota}}{\longrightarrow} \operatorname{End}(X)\left(w_{1}, \ldots, w_{r}\right)
$$


and for the identity $i d:\{1, \ldots, q\} \rightarrow\{1, \ldots, q\}$ the map ()$^{i d}: \operatorname{End}(X)\left(w ; w_{1}, \ldots, w_{q}\right) \rightarrow \operatorname{End}(X)\left(w ; w_{1}, \ldots, w_{q}\right)$ is the identity.

(4) The End $(X)$-compositions $\gamma$ are equivariant with respect to

$\operatorname{End}(X)$-functoriality in the sense that if $\iota:\{1, \ldots, p\} \rightarrow\{1, \ldots, q\}$ is a function, $\alpha \in \operatorname{End}(X)\left(w ; w_{\iota(1)}, \ldots, w_{\iota(p)}\right)$, and

$\alpha_{\ell} \in \operatorname{End}(X)\left(w_{\ell} ; w_{\ell 1}, \ldots, w_{\ell p_{\ell}}\right)$ for $\ell=1, \ldots, q$ then

$$
\gamma\left(\alpha^{\iota} ; \alpha_{1}, \ldots, \alpha_{q}\right)=\gamma\left(\alpha ; \alpha_{\iota(1)}, \ldots, \alpha_{\iota(p)}\right)^{\bar{\iota}},
$$

where $\bar{\iota}:\left\{1,2, \ldots, p_{\iota(1)}+\cdots+p_{\iota(p)}\right\} \rightarrow\left\{1,2, \ldots, p_{1}+\cdots+p_{q}\right\}$ is the function obtained by parsing the sequence $1,2, \ldots, p_{1}+\cdots+p_{q}$ into consecutive blocks $B_{1}, \ldots, B_{q}$ of lengths $p_{1}, \ldots, p_{q}$ and then writing them in the order $B_{\iota(1)}, \ldots, B_{\iota(p)}$ as in the section on theories.

(5) The End $(X)$-compositions $\gamma$ are equivariant with respect to

$\operatorname{End}(X)$-functoriality in the sense that if $\alpha \in \operatorname{End}(X)\left(w ; w_{1}, \ldots, w_{q}\right)$,

$\alpha_{\ell} \in \operatorname{End}(X)\left(w_{\ell} ; w_{\iota_{\ell}(1)}, \ldots, w_{\ell_{\ell}\left(p_{\ell}^{\prime}\right)}\right)$, and $\iota_{\ell}:\left\{1, \ldots, p_{\ell}^{\prime}\right\} \rightarrow\left\{1, \ldots, p_{\ell}\right\}$ are functions for $\ell=1, \ldots, q$ then

$$
\gamma\left(\alpha ;\left(\alpha_{1}\right)^{\iota_{1}}, \ldots,\left(\alpha_{q}\right)^{\iota_{q}}\right)=\gamma\left(\alpha ; \alpha_{1}, \ldots, \alpha_{q}\right)^{\iota_{1}+\cdots \iota_{q}}
$$

where $\iota_{1}+\cdots+\iota_{q}:\left\{1, \ldots, p_{1}^{\prime}+\cdots+p_{q}^{\prime}\right\} \rightarrow\left\{1, \ldots, p_{1}+\cdots+p_{q}\right\}$ is the function obtained by placing $\iota_{1}, \ldots, \iota_{q}$ side by side.

(6) End(I)-functoriality is functorial, i.e.

for functions $\{1, \ldots, n\} \stackrel{f}{\longrightarrow}\{1, \ldots, m\} \stackrel{g}{\longrightarrow}\{1, \ldots, \ell\}$ and words $w, w_{1}, \ldots, w_{q} \in \operatorname{Mor}_{\operatorname{End}(I)^{k}}(n, 1)$ the composition

$$
\begin{gathered}
\operatorname{End}(X)\left(w ; w_{1}, \ldots, w_{q}\right) \stackrel{()_{f}}{\longrightarrow} \operatorname{End}(X)\left(w_{f} ;\left(w_{1}\right)_{f}, \ldots,\left(w_{q}\right)_{f}\right) \\
\stackrel{()_{g}}{\longrightarrow} \operatorname{End}(X)\left(\left(w_{f}\right)_{g} ;\left(\left(w_{1}\right)_{f}\right)_{g}, \ldots,\left(\left(w_{q}\right)_{f}\right)_{g}\right)
\end{gathered}
$$

is the same as

$$
\operatorname{End}(X)\left(w ; w_{1}, \ldots, w_{q}\right) \stackrel{()_{g \circ f}}{\longrightarrow} \operatorname{End}(X)\left(w_{g \circ f} ;\left(w_{1}\right)_{g \circ f}, \ldots,\left(w_{q}\right)_{g \circ f}\right)
$$

and for the identity $i d:\{1, \ldots n\} \rightarrow\{1, \ldots, n\}$ the map ()$_{i d}: \operatorname{End}(X)\left(w ; w_{1}, \ldots, w_{q}\right) \rightarrow \operatorname{End}(X)\left(w ; w_{1}, \ldots, w_{q}\right)$ is the identity.

(7) End(I)-substitution is associative.

Let $w, w_{1}, \ldots, w_{q} \in \operatorname{Mor}_{\operatorname{End}(I)^{k}}(m, 1), t_{i} \in \operatorname{End}(I)\left(k_{i}\right)$ for $i=1, \ldots, m$ and $s_{i j} \in \operatorname{End}(I)\left(k_{i j}\right)$ for $1 \leq i \leq m$ and $1 \leq j \leq k_{i}$. Let $v:=$ $\gamma^{\times k}\left(w ; t_{1}^{\times k}, \ldots, 1_{m}^{\times k}\right), v_{\ell}:=\gamma^{\times k}\left(w_{\ell} ; t_{1}^{\times k}, \ldots, t_{m}^{\times k}\right)$,

$u:=\gamma^{\times k}\left(v ; s_{11}^{\times k}, s_{12}^{\times k}, \ldots, s_{1 k_{1}}^{\times k}, s_{21}^{\times k}, \ldots, s_{31}^{\times k}, \ldots, s_{m 1}^{\times k}, \ldots, s_{m k_{m}}^{\times k}\right)$,

$u_{\ell}:=\gamma^{\times k}\left(v_{\ell} ; s_{11}^{\times k}, s_{12}^{\times k}, \ldots, s_{1 k_{1}}^{\times k}, s_{21}^{\times k}, \ldots, s_{31}^{\times k}, \ldots, s_{m 1}^{\times k}, \ldots, s_{m k_{m}}^{\times k}\right)$ for $\ell=$ $1, \ldots, q$. Then the composition

$$
\begin{gathered}
\left.\operatorname{End}(X)\left(w ; w_{1}, \ldots, w_{q}\right) \stackrel{\left(t_{1}, \ldots, t_{m}\right)^{*}}{\longrightarrow} \operatorname{End}(X)\left(v ; v_{1}, \ldots, v_{q}\right)\right) \\
\stackrel{\left(s_{11}, \ldots, s_{m k_{m}}\right)^{*}}{\longrightarrow} \operatorname{End}(X)\left(u ; u_{1}, \ldots u_{q}\right)
\end{gathered}
$$

is the same as

$$
\operatorname{End}(X)\left(w ; w_{1}, \ldots, w_{q}\right) \stackrel{\left(r_{1}, \ldots, r_{m}\right)^{*}}{\longrightarrow} \operatorname{End}(X)\left(u ; u_{1}, \ldots, u_{q}\right)
$$


where $r_{i}=\gamma^{\times k}\left(t_{i}^{\times k} ; s_{i 1}^{\times k}, s_{i 2}^{\times k}, \ldots, s_{i k_{i}}^{\times k}\right)=\gamma_{E n d(I)}\left(t_{i} ; s_{i 1}, s_{i 2}, \ldots, s_{i k_{i}}\right)^{\times k}$ for $i=1, \ldots, m$. Note that

$u=\gamma^{\times k}\left(w ; \gamma^{\times k}\left(t_{1}^{\times k} ; s_{11}^{\times k}, s_{12}^{\times k}, \ldots, s_{1 k_{1}}^{\times k}\right), \ldots, \gamma^{\times k}\left(t_{m}^{\times k} ; s_{m 1}^{\times k}, s_{m 2}^{\times k}, \ldots, s_{m k_{m}}^{\times k}\right)\right)$.

(8) End(I)-substitution is unital.

For the unit $1 \in \operatorname{End}(I)(1)$ of the theory $\operatorname{End}(I)$ and $w, w_{1}, \ldots, w_{q} \in \operatorname{Mor}_{\operatorname{End}(I)^{k}}(m, 1)$ the function

$$
(1, \ldots, 1)^{*}: \operatorname{End}(X)\left(w ; w_{1}, \ldots, w_{q}\right) \rightarrow \operatorname{End}(X)\left(w ; w_{1}, \ldots, w_{q}\right)
$$

is the identity.

(9) $\operatorname{End}(X)$-composition is End $(I)$-equivariant.

If $f:\{1, \ldots, m\} \rightarrow\{1, \ldots, \ell\}$ is a function, $w, w_{i}, w_{i j} \in \operatorname{Mor}_{\operatorname{End}(I)^{k}}(m, 1)$, $\alpha \in \operatorname{End}(X)\left(w ; w_{1}, \ldots w_{q}\right), \alpha_{j} \in \operatorname{End}(X)\left(w_{j} ; w_{j 1}, \ldots, w_{j p_{j}}\right)$ for $j=1, \ldots, q$, then

$$
\gamma\left(\alpha_{f} ;\left(\alpha_{1}\right)_{f}, \ldots,\left(\alpha_{q}\right)_{f}\right)=\left(\gamma\left(\alpha ; \alpha_{1}, \ldots, \alpha_{q}\right)\right)_{f} .
$$

(10) End $(X)$-functoriality and End(I)-functoriality commute.

For functions $\iota:\{1, \ldots, p\} \rightarrow\{1, \ldots, q\}$ and $f:\{1, \ldots, m\} \rightarrow\{1, \ldots, \ell\}$ and morphism $\alpha \in \operatorname{End}(X)\left(w ; w_{\iota(1)}, \ldots, w_{\iota(p)}\right)$ we have $\left(\alpha^{\iota}\right)_{f}=\left(\alpha_{f}\right)^{\iota}$.

(11) End $(X)$-functoriality and End(I)-substitution commute.

The diagram

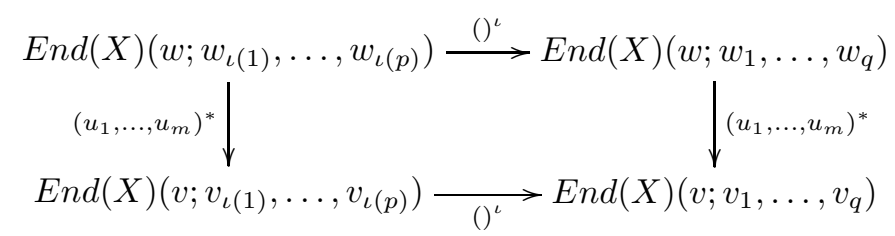

commutes.

(12) End(I)-functoriality and End(I)-substitution commute, in the sense that if $f_{i}:\left\{1, \ldots, k_{i}\right\} \rightarrow\left\{1, \ldots, k_{i}^{\prime}\right\}$ are functions and $u_{i} \in \operatorname{End}(I)\left(k_{i}\right)$ for $i=1, \ldots, m$ and $w, w_{1}, \ldots, w_{q} \in \operatorname{End}(I)^{k}(m)$, then

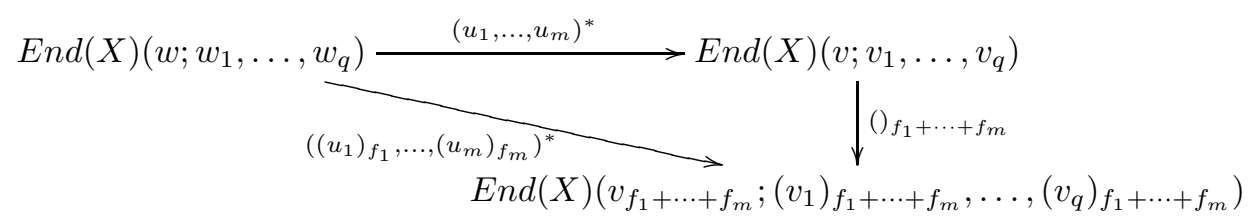

commutes. Note that

$$
\begin{aligned}
\gamma^{\times k}\left(w ;\left(u_{1}\right)_{f_{1}}^{\times k}, \ldots,\left(u_{m}\right)_{f_{m}}^{\times k}\right) & =\gamma^{\times k}\left(w ; u_{1}, \ldots, u_{m}\right)_{f_{1}+\cdots+f_{m}} \\
& =v_{f_{1}+\cdots f_{m}} .
\end{aligned}
$$

(13) End(I)-functoriality and End(I)-substitution commute, in the sense that if $f:\{1, \ldots, m\} \rightarrow\{1, \ldots, \ell\}$ is a function and $u_{i} \in \operatorname{End}(I)\left(k_{i}\right)$ for $i=$ $1, \ldots, \ell$, then the diagram

$$
\begin{gathered}
\operatorname{End}(X)\left(w ; w_{1}, \ldots, w_{q}\right) \stackrel{()_{f}}{\longrightarrow} \operatorname{End}(X)\left(w_{f} ;\left(w_{1}\right)_{f}, \ldots,\left(w_{q}\right)_{f}\right) \\
\left(u_{f 1}, \ldots, u_{f m}\right)^{*} \downarrow \\
\operatorname{End}(X)\left(v ; v_{1}, \ldots, v_{q}\right) \underset{\left(u_{1}, \ldots, u_{\ell}\right)^{*}}{\stackrel{()_{\bar{f}}}{\longrightarrow}} \operatorname{End}(X)\left(v_{\bar{f}} ;\left(v_{1}\right)_{\bar{f}}, \ldots,\left(v_{q}\right)_{\bar{f}}\right)
\end{gathered}
$$


commutes, where $v=\gamma^{\times k}\left(w ; u_{f 1}, \ldots, u_{f m}\right)$ and $v_{\bar{f}}=\gamma^{\times k}\left(w_{f} ; u_{1}, \ldots, u_{\ell}\right)$ etc.

(14) End(I)-substitution and End(I)-composition commute.

Let $w, w_{i}, w_{i j} \in \operatorname{Mor}_{\operatorname{End}(I)^{k}}(m, 1)$ and $u_{i} \in \operatorname{End}(I)\left(k_{i}\right)$ for $i=1, \ldots, m$. Let $\alpha \in \operatorname{End}(X)\left(w ; w_{1}, \ldots, w_{q}\right), \alpha_{\ell} \in \operatorname{End}(X)\left(w_{\ell} ; w_{\ell 1}, \ldots, w_{\ell p_{\ell}}\right)$ for $\ell=$ $1, \ldots, q$ and $\beta:=\left(u_{1}, \ldots, u_{m}\right)^{*} \alpha$ etc. Then

$$
\left(u_{1}, \ldots, u_{m}\right)^{*} \gamma\left(\alpha ; \alpha_{1}, \ldots, \alpha_{q}\right)=\gamma\left(\beta ; \beta_{1}, \ldots, \beta_{q}\right) .
$$

12.2. 2-Theories and Algebras over 2-Theories. A general 2-theory has all of the properties described in the example above. P. Hu and I. Kriz introduce the notion of a 2-theory in 24] as follows.

Definition 12.1. A 2-theory $(\Theta, T)$ fibered over the theory $T$ is a natural number $k$, a theory $T$, and a contravariant functor $\Theta: T \rightarrow$ Cat from the category $T$ to the 2-category $C a t$ of small categories such that

- $\operatorname{Obj} \Theta(m)=\coprod_{n \geq 0} \operatorname{Hom}_{T^{k}}(m, n)$ for all $m \in \mathbb{N}$, where $T^{k}$ is the theory with the same objects as $T$, but with $\operatorname{Hom}_{T^{k}}(m, n)=\operatorname{Hom}_{T}(m, n)^{k}$

- If $w_{1}, \ldots, w_{n} \in \operatorname{Hom}_{T^{k}}(m, 1)$, then the word in $\operatorname{Hom}_{T^{k}}(m, n)$ with which the $n$-tuple is identified is the product in $\Theta(m)$ of $w_{1}, \ldots, w_{n}$

- For $w \in \operatorname{Hom}_{T}(m, n)$ the functor $\Theta(w): \Theta(n) \rightarrow \Theta(m)$ is $\Theta(w)(v)=$ $v \circ w^{\times k}$ on objects $v \in \operatorname{Hom}_{T^{k}}(n, j)$.

For objects $w_{1}, \ldots, w_{n}, w \in \operatorname{Mor}_{T^{k}}(m, 1) \subseteq \operatorname{Obj} \Theta(m)$ we set

$$
\Theta\left(w ; w_{1}, \ldots, w_{n}\right):=\operatorname{Mor}_{\Theta(m)}\left(\prod_{i=1}^{n} w_{i}, w\right) .
$$

The second condition explains the choice of notation $\prod_{i=1}^{n} w_{i}$. Given a 2-theory such as this, it has operations and relations as in Theorem 12.1 Vice-a-versa, given sets $\Theta\left(w ; w_{1}, \ldots, w_{n}\right):=\operatorname{Mor}_{\Theta(m)}\left(\prod_{i=1}^{n} w_{i}, w\right)$ with operations and relations as in Theorem 12.1 we get a 2-theory. We refer to these operations and relations as the operations and relations of a 2-theory. Recall that a pseudo algebra $I$ over the theory $T$ is a category such that for every word $w \in T(n)$ we have a functor $\Phi(w): I \times \cdots \times I \rightarrow I$. Moreover, for every operation of the theory $T$ (identity, composition, substitution) we have a coherence iso and for every relation among the operations we have a coherence diagram. A pseudo $(\Theta, T)$-algebra can be defined analogously.

Definition 12.2. Let $(\Theta, T)$ be a 2-theory. A pseudo $(\Theta, T)$-algebra consists of the following data:

- a small pseudo $T$-algebra $I$ with action $\Phi: T(n) \rightarrow F$ unctors $\left(I^{n}, I\right)$

- a strict 2-functor $X: I^{k} \rightarrow$ Cat

- set maps $\phi: \Theta\left(w ; w_{1}, \ldots, w_{n}\right) \rightarrow \operatorname{End}(X)\left(\Phi(w) ; \Phi\left(w_{1}\right), \ldots, \Phi\left(w_{n}\right)\right)$, where $\Phi(w)$ means to apply $\Phi$ to each component of $w$ to make $I^{k}$ into the product pseudo algebra of $k$ copies of $I$.

- a coherence iso for each operation of a 2-theory.

The only requirement on these data is that the coherence isos satisfy the coherence diagrams which come from the relations of a 2-theory.

A morphism of pseudo $(\Theta, T)$-algebras is similar to a morphism of pseudo $T$ algebras. 
Definition 12.3. Let $X, Y: I^{k} \rightarrow C a t$ be pseudo $(\Theta, T)$-algebras over $I^{k}$. Then a morphism $H: X \rightarrow Y$ is a strict natural transformation $H: X \Rightarrow Y$ with coherence iso modifications $\rho_{\alpha}$ indexed by elements $\alpha \in \Theta\left(w ; w_{1}, \ldots, w_{n}\right)$, where $w, w_{1}, \ldots, w_{n} \in \operatorname{Obj} \Theta(m)$.

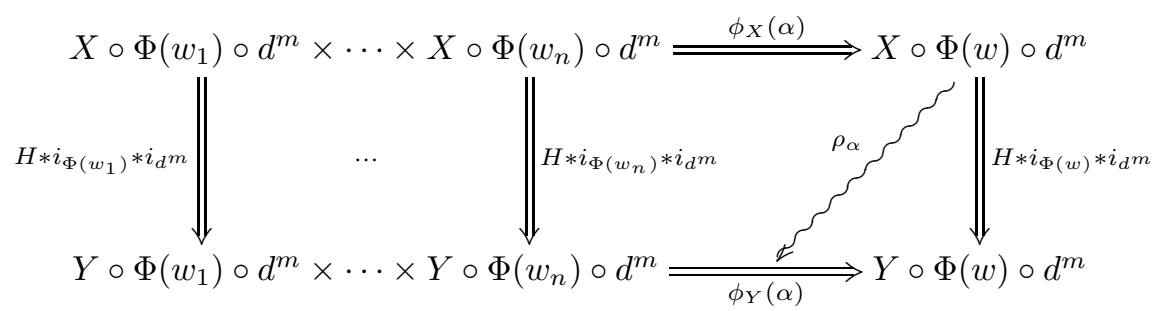

The coherence iso modification $\rho_{\alpha}$ is also required to commute with all coherence iso modifications of the pseudo algebra structure.

The 2-category of pseudo $(\Theta, T)$-algebras over $I^{k}$ must also have 2-cells.

Definition 12.4. Let $G, H: X \rightarrow Y$ be morphisms as above. Then a 2-cell $\sigma: G \Rightarrow H$ is a modification which commutes with the coherence iso modifications $\rho^{G}$ and $\rho^{H}$ appropriately.

Theorem 12.3. The pseudo $(\Theta, T)$-algebras over $I^{k}$ form a 2-category.

Proof: Routine.

12.3. The Algebraic Structure of Rigged Surfaces. The purpose of this subsection is to introduce the category $\mathcal{C}$ of rigged surfaces as an example of a pseudo algebra over a 2-theory fibered over a theory and to describe its stack structure. This approach is introduced in 24] by P. Hu and I. Kriz. In their terminology, a smooth, compact, not necessarily connected, 2-dimensional manifold $x$ with a complex structure is called a rigged surface if each boundary component $k$ comes equipped with a parametrization diffeomorphism $f_{k}: S^{1} \rightarrow k$ which is analytic with respect to the complex structure on $x$, i.e. the diffeomorphism $f_{k}$ extends to a holomorphic map when we go into local coordinates. A boundary component $k$ is called inbound or outbound depending on the orientation of its parametrization $f_{k}$ with respect to the orientation on $k$ induced by the complex structure. The convention is to call the identity parametrization of the boundary of the unit disk inbound. A morphism of rigged surfaces is a smooth map which preserves the complex structure and boundary parametrizatons.

The structure of the category $\mathcal{C}$ of rigged surfaces has the following features, which were studied in 24. For finite sets $a$ and $b$, let $\operatorname{Obj} X_{a, b}$ denote the set of rigged surfaces $x$ equipped with a bijection between the inbound boundary components of $x$ and $a$ as well as a bijection between the outbound boundary components of $x$ and $b$. For $x, y \in X_{a, b}$ let $\operatorname{Mor}_{X_{a, b}}(x, y)$ be the morphisms of rigged surfaces which preserve the bijections with $a$ and $b$. For finite sets $a, b, c$, and $d$ we can take the disjoint union of any two rigged surfaces $x \in \operatorname{Obj} X_{a, b}$ and $y \in \operatorname{Obj} X_{c, d}$ and the result is an element of $\operatorname{Obj} X_{a} \amalg c, b \amalg d$. One can apply this process to morphisms as well, and we get a functor $\amalg: X_{a, b} \times X_{c, d} \rightarrow X_{a \amalg c, b \amalg d}$ called disjoint union. Note that this functor is indexed by the finite sets $a, b, c$, and $d$. For finite sets 
$a, b$, and $c$ we also have a gluing functor $\stackrel{?}{:}: X_{a} \amalg c, b \amalg c \rightarrow X_{a, b}$ which identifies certain inbound boundary components with certain outbound boundary components according to the bijections with $a \coprod c$ and $b \coprod c$ as well as the parametrizations of the relevant boundary components in the manner prescribed by $f(z) \sim f^{\prime}(z)$ for all $z \in S^{1}$. This gluing functor is also indexed by the finite sets $a, b$, and $c$. These disjoint union and gluing functors along with their coherences give the category of rigged surfaces the structure of a pseudo algebra over the 2-theory of commutive monoids with cancellation.

We define the 2-theory $(\Theta, T)$ of commutative monoids with cancellation as follows. Let $T$ be the theory of commutative monoids and let $+: 2 \rightarrow 1$ and $0: 0 \rightarrow 1$ be the usual words in the theory of commutative monoids. Let $k=2$. The 2-theory $\Theta$ is generated by three words: addition + , unit 0 , and cancellation ?. These are described in terms of a general algebra $X$ over $(\Theta, T)$ as follows. Note that + and 0 have two meanings.

$$
\begin{gathered}
+: X_{a, b} \times X_{c, d} \rightarrow X_{a+c, b+d} \\
0 \in X_{0,0} \\
\check{?}: X_{a+c, b+c} \rightarrow X_{a, b}
\end{gathered}
$$

These generating words must satisfy the following axioms.

(1) The word + is commutative.

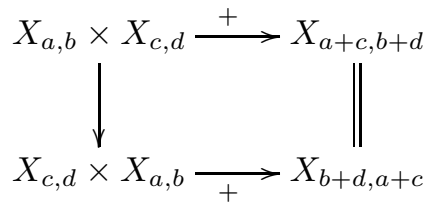

(2) The word + is associative.

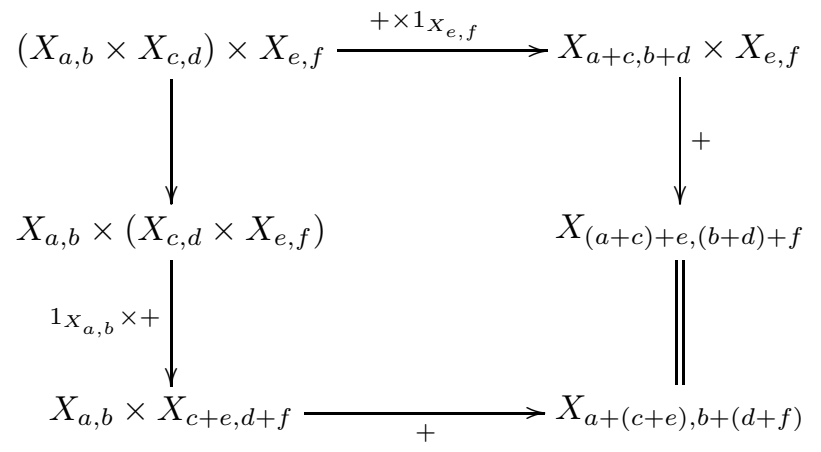

(3) The word + has unit $0 \in X_{0,0}$.

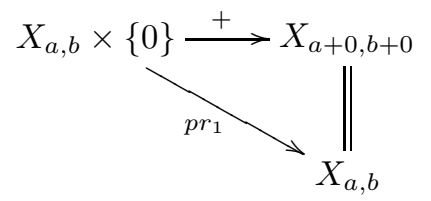


(4) The word ? is transitive.

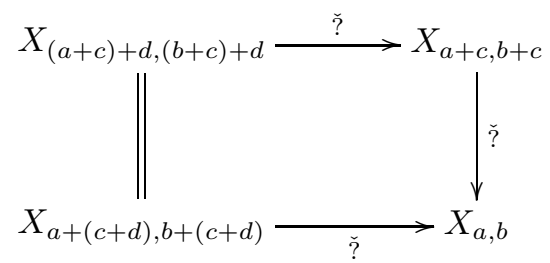

(5) The word ? distributes over the word + .

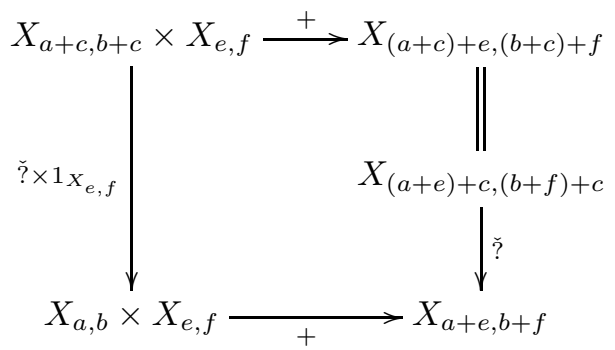

The category of rigged surfaces forms a pseudo algebra over this 2-theory of commutative monoids with cancellation. The pseudo algebra structure is given by assigning a fixed choice of $\amalg$ to + and by assigning gluing of manifolds to ?

In 24] and 25] the algebraic structure of holomorphic families of rigged surfaces is captured by a stack of pseudo algebras over the 2-theory of commutative monoids with cancellation, which is also called a stack of lax commutative monoids with cancellation (SLCMC). I describe this stack now. Let $\mathcal{B}$ be the category of finite dimensional complex manifolds with morphisms holomorphic maps. A collection $\left\{B_{i} \rightarrow B\right\}_{i}$ of (open) holomorphic embeddings are a cover if their images cover $B$. This makes $\mathcal{B}$ into a Grothendieck topology. For any finite dimensional complex manifold $B$ let $I^{B}$ denote the category of covering spaces of $B$ with finite fibers. The category $I^{B}$ is a pseudo commutative monoid. Let $s$ and $t$ be objects of $\mathcal{B}$. Define $X_{s, t}^{B}$ as the category of holomorphic families of rigged surfaces over $B$ with inbound boundary components labelled by the covering space $s$ of $B$ and outbound boundary components labelled by the covering space $t$ of $B$. Such a holomorphic family $x$ is by definition a family of rigged surfaces parameterized by $B$ and a holomorphic map $q: y \rightarrow B$ transverse to every point, where $y$ denotes the family of closed 2-dimensional manifolds obtained from $x$ by sewing disks onto the boundary components of the rigged surfaces of $x$ according to the boundary parameterizations. The holomorphic map $q$ is an extension of the parametrization of $x$. To say that the inbound boundary components of $x$ are labelled by the covering space $s$ means that for each $b \in B$ the rigged surface $x_{b}$, which is the fiber of $\left.q\right|_{x}$ over $b$, is equipped with a bijection between its inbound boundary components and the fiber of $s$ over $b$. The explanation for the covering space $t$ labelling the outbound boundary components is similar. With these definitions as well as disjoint union and gluing, the functor $X^{B}:\left(I^{B}\right)^{2} \rightarrow$ Cat is a pseudo algebra over the 2-theory of commutative monoids with cancellation.

Let $\mathcal{C}$ denote the 2-category of pseudo algebras over the 2-theory of commutative monoids with cancellation. This 2-category admits bilimits and a simpler case of this is proved in the next subsection. Define a contravariant pseudo functor 
$G: \mathcal{B} \rightarrow \mathcal{C}$ by taking a finite dimensional manifold to the pseudo algebra $X^{B}$ over the 2-theory of pseudo commutative monoids with cancellation with underlying pseudo commutative monoid $I^{B}$. Then $G$ takes Grothendieck covers to bilimits because it does so on the underlying categories comprising the pseudo algebras. Hence $G$ is a stack. It is in this sense that the category $\mathcal{C}$ of rigged surfaces forms a stack.

12.4. Weighted Pseudo Limits of Pseudo $(\Theta, T)$-algebras. The 2-category of pseudo $(\Theta, T)$-algebras admits all weighted pseudo limits, just like the 2-category of pseudo $T$-algebras. In the following theorem we prove this for pseudo $(\Theta, T)$ algebras with fixed underlying pseudo $T$-algebra $I^{k}$. The proof can be modified to the general case of pseudo $(\Theta, T)$-algebras with different underlying pseudo $T$ algebras by taking the pseudo limit of the underlying pseudo $T$-algebras as well.

Theorem 12.4. Let $\mathcal{J}$ be a 1-category and $\mathcal{C}$ the 2-category of pseudo $(\Theta, T)$ algebras over $I^{k}$. Let $F: \mathcal{J} \rightarrow \mathcal{C}$ be a pseudo functor. Then $F$ has a pseudo limit $(X, \pi)$ in $\mathcal{C}$, where $\pi: \Delta_{X} \Rightarrow F$ is a universal pseudo cone.

Proof: $\quad$ Let $\gamma$ and $\delta$ be the 2-cells in $\mathcal{C}$ which make $F$ into a pseudo functor. They satisfy the usual coherences. For each $j \in O b j \mathcal{J}$, let $X^{j}: I^{k} \rightarrow C a t$ be the strict 2-functor belonging to the pseudo $(\Theta, T)$-algebra $F j$. Then for each fixed object $i \in O b j I^{k}$ and each object $j \in \mathcal{J}$ we have a category $X_{i}^{j}$. For each morphism $f: j \rightarrow m$ in $\mathcal{J}$, the map $F f: X^{j} \Rightarrow X^{m}$ is a strict 2-natural transformation which gives us a functor $(F f)_{i}: X_{i}^{j} \rightarrow X_{i}^{m}$ for each $i \in \operatorname{ObjI}^{k}$. Thus for fixed $i$ we have a pseudo functor $F_{i}: \mathcal{J} \rightarrow C$ at defined by $j \mapsto X_{i}^{j}$ and $f \mapsto(F f)_{i}$. The coherence isos of $F_{i}$ are the coherence iso modifications of $F$ evaluated at $i$.

Let $X_{i}:=$ PseudoCone $\left(\mathbf{1}, F_{i}\right)$, where $\mathbf{1}$ is the terminal object in the category of small categories. Then it is known from Section 4 that $X_{i}$ is the pseudo limit of $F_{i}$ in Cat. Proceeding analogously on morphisms of $I^{k}$, we obtain a strict 2-functor $X: I^{k} \rightarrow C a t$ defined by $i \mapsto X_{i}$. More precisely, if $h: i_{1} \rightarrow i_{2}$ is a morphism of $I^{k}$ and $\eta \in \operatorname{Obj} X_{i_{1}}$, then $X_{h}(\eta)(j):=X_{h}^{j}(\eta(j))$ for $j \in O b j \mathcal{J}$.

A more conceptual way to view the construction of the strict 2-functor $X: I^{k} \rightarrow$ Cat is the following. For $i \in I^{k}$, let $F_{i}: \mathcal{J} \rightarrow$ Cat be the pseudo functor from above. For a morphism $h: i_{1} \rightarrow i_{2}$ in $I^{k}$, let $F_{h}: F_{i_{1}} \Rightarrow F_{i_{2}}$ be the pseudo natural transformation given by $F_{h}(j):=X_{h}^{j}$. The pseudo natural transformation $F_{h}$ is actually strictly 2-natural because $F f: X^{j} \Rightarrow X^{m}$ is a strict 2-natural transformation for each $f: j \rightarrow m$ in $\mathcal{J}$. Thus $i \mapsto F_{i}$ and $h \mapsto F_{h}$ define a strict functor $I^{k} \rightarrow$ Functors $(\mathcal{J}, C a t)$. Now recall that PseudoCone $(\mathbf{1},-)$ is a covariant functor from Functors $(\mathcal{J}$, Cat $)$ to Cat. The composition

$$
I^{k} \longrightarrow \text { Functors }(\mathcal{J}, \text { Cat }) \stackrel{\text { PseudoCone }(\mathbf{1},-)}{\longrightarrow} \text { Cat }
$$

is $X: I^{k} \rightarrow$ Cat.

I claim that this 2-functor $X: I^{k} \rightarrow$ Cat has the structure of a pseudo $(\Theta, T)$ algebra. The argument is like Lemma 7.2 although the coherences need some care. First I define maps $\phi: \Theta\left(w ; w_{1}, \ldots, w_{n}\right) \rightarrow \operatorname{End}(X)\left(\Phi(w) ; \Phi\left(w_{1}\right), \ldots, \Phi\left(w_{n}\right)\right)$, where $w_{1}, \ldots, w_{n}, w \in \operatorname{Hom}_{T^{k}}(m, 1)$. Let $\alpha \in \Theta\left(w ; w_{1}, \ldots, w_{n}\right)$. We need to define a natural transformation

$$
\phi(\alpha): X \circ \Phi\left(w_{1}\right) \circ d^{m} \times \cdots \times X \circ \Phi\left(w_{n}\right) \circ d^{m} \Rightarrow X \circ \Phi(w) \circ d^{m}
$$


"componentwise," where $d^{m}: I^{m} \rightarrow\left(I^{m}\right)^{k}$ is the diagonal functor. Let $\phi_{j}$ : $\Theta\left(w ; w_{1}, \ldots, w_{n}\right) \rightarrow \operatorname{End}\left(X^{j}\right)\left(\Phi(w) ; \Phi\left(w_{1}\right), \ldots, \Phi\left(w_{n}\right)\right)$ be the maps that make $X^{j}: I^{k} \rightarrow$ Cat into a pseudo $(\Theta, T)$-algebra for each $j \in O b j \mathcal{J}$. Let $i \in I^{m}$. We define a functor

$$
(\phi(\alpha))_{i}: X_{\Phi\left(w_{1}\right) \circ d^{m}(i)} \times \cdots \times X_{\Phi\left(w_{n}\right) \circ d^{m}(i)} \rightarrow X_{\Phi(w) \circ d^{m}(i)}
$$

and show that $i \mapsto(\phi(\alpha))_{i}$ is natural. Recall that objects of $X_{\Phi\left(w_{\ell}\right) \circ d^{m}(i)}=$ PseudoCone $\left(\mathbf{1}, F_{\Phi\left(w_{\ell}\right) \circ d^{m}(i)}\right)$ can be identified with a subset of

$$
\begin{gathered}
\left\{\left(a_{j}\right)_{j} \times\left(\varepsilon_{f}\right)_{f} \in \prod_{j \in O b j \mathcal{J}} \operatorname{Obj}_{\Phi\left(w_{\ell}\right) \circ d^{m}(i)}^{j} \times \prod_{f \in M o r \mathcal{J}} \operatorname{Mor} X_{\Phi\left(w_{\ell}\right) \circ d^{m}(i)}^{T f} \mid\right. \\
\left.\varepsilon_{f}:(F f)_{\Phi\left(w_{\ell}\right) \circ d^{m}(i)}\left(a_{S f}\right) \rightarrow a_{T f} \text { is iso for all } f \in M o r \mathcal{J}\right\}
\end{gathered}
$$

by Remark 4.4 A similar statement holds for morphisms according to 4.5] Let $\eta^{\ell}=\left(a_{j}^{\ell}\right)_{j} \times\left(\varepsilon_{f}^{\ell}\right)_{f} \in O \operatorname{Obj} X_{\Phi\left(w_{\ell}\right) \circ d^{m}(i)}$ and $\left(\xi_{j}^{\ell}\right)_{j} \in \operatorname{Mor} X_{\Phi\left(w_{\ell}\right) \circ d^{m}(i)}$ for $1 \leq \ell \leq n$. Define

and

$$
a_{j}:=\left(\phi_{j}(\alpha)\right)_{i}\left(a_{j}^{1}, \ldots, a_{j}^{n}\right)
$$

Note that

$$
\varepsilon_{f}:=\left(\phi_{T f}(\alpha)\right)_{i}\left(\varepsilon_{f}^{1}, \ldots, \varepsilon_{f}^{n}\right) \circ\left(\rho_{\alpha}^{F f}\right)_{i}\left(a_{S f}^{1}, \ldots, a_{S f}^{n}\right) .
$$

$$
\begin{gathered}
\left(\rho_{\alpha}^{F f}\right)_{i}\left(a_{S f}^{1}, \ldots, a_{S f}^{n}\right):(F f)_{\Phi\left(w_{\ell}\right) \circ d^{m}(i)}\left(\phi_{S f}(\alpha)\right)_{i}\left(a_{S f}^{1}, \ldots, a_{S f}^{n}\right) \rightarrow \\
\quad\left(\phi_{T f}(\alpha)\right)_{i}\left((F f)_{\Phi\left(w_{\ell}\right) \circ d^{m}(i)}\left(a_{S f}^{1}\right), \ldots,(F f)_{\Phi\left(w_{\ell}\right) \circ d^{m}(i)}\left(a_{S f}^{n}\right)\right)
\end{gathered}
$$

and the composition in the definition of $\varepsilon_{f}$ makes sense. Also define

$$
\xi_{j}:=\left(\phi_{j}(\alpha)\right)_{i}\left(\xi_{j}^{1}, \ldots, \xi_{j}^{n}\right) .
$$

Then the action of $\alpha$ on $X$ is defined "componentwise" by

$$
(\phi(\alpha))_{i}\left(\eta^{1}, \ldots, \eta^{n}\right):=\left(a_{j}\right)_{j} \times\left(\varepsilon_{f}\right)
$$

and

$$
(\phi(\alpha))_{i}\left(\left(\xi_{j}^{1}\right)_{j}, \ldots,\left(\xi_{j}^{n}\right)_{j}\right):=\left(\xi_{j}\right)_{j}
$$

By an argument similar to the proof of Lemma 7.2 these images are actually in $X_{\Phi(w) \circ d^{m}(i)}$. Next note that $i \mapsto(\phi(\alpha))_{i}$ is natural because $i \mapsto\left(\phi_{j}(\alpha)\right)_{i}$ is natural for all $j \in O b j \mathcal{J}$, i.e. $i \mapsto(\phi(\alpha))_{i}$ is natural in each "coordinate" and is therefore natural. Hence we have constructed set maps $\phi: \Theta\left(w ; w_{1}, \ldots, w_{n}\right) \rightarrow \operatorname{End}(X)\left(\Phi(w) ; \Phi\left(w_{1}\right), \ldots, \Phi\left(w_{n}\right)\right)$.

We define the coherence iso modifications for $\phi$ to be those modifications which have the coherence iso modifications for $\phi_{j}$ in the $j$-th coordinate. For example, we define the identity modification $I_{w}: 1_{\Phi(w)} \rightsquigarrow \phi\left(1_{w}\right)$ by

$$
I_{w}\left(\left(a_{j}\right)_{j} \times\left(\varepsilon_{f}\right)_{f}\right):=\left(I_{w}^{j}\left(a_{j}\right)\right)_{j}
$$

for $i \in I^{m}$ and $\left(a_{j}\right)_{j} \times\left(\varepsilon_{f}\right)_{f} \in X_{\Phi(w) \circ d^{m}(i)}$. The arrow $I_{w}\left(\left(a_{j}\right)_{j} \times\left(\varepsilon_{f}\right)_{f}\right)$ is an arrow in the category $X_{\Phi(w) \circ d^{m}(i)}$ by an argument like the proof of Lemma 7.2 Similarly, one can show that these assignments are modifications and that the coherence diagrams are satisfied because everything is done componentwise. Hence $X: I^{k} \rightarrow C a t$ has the structure of a pseudo $(\Theta, T)$-algebra.

Next we need a universal pseudo cone $\pi: \Delta_{X} \Rightarrow F$, where $\Delta_{X}: \mathcal{J} \rightarrow \mathcal{C}$ is the constant functor which takes everything to $X$. Define a natural transformation $\pi_{j}$ : $X \Rightarrow X^{j}$ by letting $\pi_{j}(i): X_{i} \Rightarrow X_{i}^{j}$ be the projection. The natural transformation 
$\pi_{j}$ commutes with the $(\Theta, T)$ action, and so $\pi_{j}$ is a morphism of pseudo $(\Theta, T)$ algebras by taking the coherence iso modifications to be trivial. The assignment $j \mapsto \pi_{j}$ is pseudo natural with coherence 2-cell $\tau_{j, m}(f): F f \circ \pi_{j} \Rightarrow \pi_{m}$ for each $f: j \rightarrow m$ in $\mathcal{J}$ as in the 1-theory case. A similar argument to the 1-theory case shows that $\tau_{j, m}(f)$ is a 2 -cell in $\mathcal{C}$. Hence, we have a pseudo natural transformation $\pi: \Delta_{X} \Rightarrow F$. One can prove the universality of $\pi$ by applying the argument in the lemmas leading up to Theorem [7.9] to $X_{i} \rightarrow X_{i}^{j}$ for each fixed $i \in \operatorname{ObjI}^{k}$ and then passing to functors $I^{k} \rightarrow$ Cat. One must of course take the coherence isos into consideration.

We conclude that $(X, \pi)$ is a pseudo limit of the pseudo functor $F: \mathcal{J} \rightarrow \mathcal{C}$.

Theorem 12.5. The 2-category of pseudo $(\Theta, T)$-algebras over $I^{k}$ admits pseudo limits.

Proof: This follows immediately from the previous theorem.

Lemma 12.6. The 2-category $\mathcal{C}$ of pseudo $(\Theta, T)$-algebras admits cotensor products.

Proof: $\quad$ Let $J \in O b j C a t$ and let $F: I^{k} \rightarrow$ Cat be a pseudo $(\Theta, T)$-algebra. Define a strict 2-functor $P: I^{k} \rightarrow C a t$ by $P_{i}:=\left(F_{i}\right)^{J}$, which is the 1-category of 1functors $J \rightarrow F_{i}$. I claim that $P$ has the structure of a pseudo $(\Theta, T)$-algebra. This structure is obtained by doing the operations pointwise. Let $\phi: \Theta\left(w ; w_{1}, \ldots, w_{n}\right) \rightarrow$ $\operatorname{End}(F)\left(\Phi(w) ; \Phi\left(w_{1}\right), \ldots, \Phi\left(w_{n}\right)\right)$ denote the maps which make $F$ into a pseudo $(\Theta, T)$-algebra. Then define

$\phi^{P}: \Theta\left(w ; w_{1}, \ldots, w_{n}\right) \rightarrow \operatorname{End}(P)\left(\Phi(w) ; \Phi\left(w_{1}\right), \ldots, \Phi\left(w_{n}\right)\right)$ by

$$
\phi^{P}(\alpha)_{i}\left(\eta^{1}, \ldots, \eta^{n}\right)(j):=\phi(\alpha)_{i}\left(\eta^{1}(j), \ldots, \eta^{n}(j)\right)
$$

for functors $\eta^{\ell}: J \rightarrow X_{\Phi\left(w_{\ell}\right) \circ d^{m}(i)}$ with $1 \leq \ell \leq n$. Coherence isos can also be defined in this manner. Then the coherence diagrams commute because they commute pointwise. Hence $P$ is a pseudo $(\Theta, T)$-algebra.

A proof similar to the proof of Lemma 7.12 shows that $P$ is the cotensor product of $J$ and $F$. One must apply the argument for $F$ in Lemma 7.12 to each $F_{i}$ for $i \in \operatorname{Obj} I^{k}$.

Theorem 12.7. The 2-category $\mathcal{C}$ of pseudo $(\Theta, T)$-algebras admits all weighted pseudo limits.

Proof: By Theorem [12.5 it admits pseudo limits, and hence it admits pseudo equalizers. The 2 -category $\mathcal{C}$ obviously admits products. By Lemma 12.6 it admits cotensor products. Hence by Theorem 2.4 it admits all weighted pseudo limits.

\section{REFERENCES}

[1] J. Adámek, F.W. Lawvere, and J. Rosický: How Algebraic is Algebra?, Theory Appl. Categ 8 (2001), 253-283

[2] J. Adámek, F.W. Lawvere, and J. Rosický: Continuous Categories Revisited, Theory Appl. Categ 11 (2003), 252-282

[3] J. Adámek, F.W. Lawvere, and J. Rosický: On the Duality Between Varieties and Algebraic Theories, Algebra Universalis 49 (2003), no. 1, 35-49

[4] J. Baez: Categorification, Higher Category Theory, Contemporary Mathematics 230 (1998), AMS 1-36 
[5] J. Baez and J. Dolan: Higher-dimensional Algebra and Topological Quantum Field Theory, Jour. of Math. Phys. 36 (1995), 6073-6105

[6] J. Bénabou: Introduction to Bicategories, in Reports of the Midwest Category Seminar, eds. Bénabou et al, Lecture Notes in Mathematics 37, Springer, 1967, pp 1-77

[7] J. Bénabou: Structures Algébriques dans les Catégories, Cahiers de Topologie et Géometrie Differéntielle, 10 (1968), 1-126

[8] G.J. Bird, G.M. Kelly, A. J. Power, and R. H. Street: Flexible Limits for 2-Categories, J. Pure Appl. Algebra 61 (1989), 1-27

[9] R. Blackwell, G.M. Kelly, and A.J. Power: Two-Dimensional Monad Theory, J. Pure Appl. Algebra 59 (1989), 1-41

[10] F. Borceux and G.M. Kelly: A Notion of Limit for Enriched Categories, Bull. Austral. Math. Soc 12 (1975), 49-72

[11] F. Borceux: Handbook of Categorical Algebra 1, Cambridge University Press, 1994

[12] L. Breen: On The Classification of 2-Gerbes and 2-Stacks, Astèrisque 225, 1994, pp. 1-160

[13] A. Carboni, S. Johnson, R. Street, and D. Verity: Modulated Bicategories, J. Pure Appl. Algebra 94 (1994), 229-282

[14] G. Dunn: Lax Operad Actions and Coherence for Monoidal $n$-Categories, $A_{\infty}$ Rings and Modules, Theory and Applications of Categories 3 (1997), No. 4, 50-84

[15] B. Fantechi: Stacks for Everybody, preprint, http://www.cgtp.duke.edu/ drm/PCMI2001

[16] R. Gordon, A. J. Power, R. Street: Coherence for Tricategories, Memoirs of the American Mathematical Society 117, no. 558, 1995

[17] J. Giraud: Cohomologie non abélienne, Springer Verlag, 1971

[18] J.W. Gray: Formal Category Theory: Adjointness for 2-Categories, Lecture Notes in Mathematics 391, Springer Verlag, Berlin 1974

[19] J.W. Gray: Quasi-Kan Extensions for 2-Categories, Bull. Amer. Math. Soc. 80 (1974), 142147

[20] J.W. Gray: The Existence and Construction of Lax Limits, Cahiers Top. et Geom. Diff. 21 (1980), 277-304

[21] J.W. Gray: Closed Categories, Lax Limits, and Homotopy Limits, J. Pure Appl. Algebra 19 (1980), 127-158

[22] J.W. Gray: The Representation of Limits, Lax Limits, and Homotopy Limits as Sections, Mathematical Applications of Category Theory, Contemporary Mathematics 30 (1984), AMS 63-83

[23] C. Hermida: From Coherent Structures to Universal Properties, J. Pure and Appl. Algebra, 165 (2001), 7-61

[24] P. Hu and I. Kriz: Conformal Field Theory and Elliptic Cohomology, to appear in Advances in Mathematics

[25] P. Hu and I. Kriz: Closed and Open Conformal Field Theories and Their Anomalies, to appear

[26] P. Hu, I. Kriz, A. Voronov: On Kontsevich's Hochschild Cohomology Conjecture, to appear

[27] G.M. Kelly and R. Street: Review of the Elements of 2-Categories, Lecture Notes in Mathematics 420, Springer Verlag, New York 1974

[28] G.M. Kelly: Basic Concepts of Enriched Category Theory, London Mathematical Society Lecture Note Series 64, Cambridge University Press, New York 1982

[29] G.M. Kelly: Elementary Observations on 2-Categorical Limits, Bull. Austral. Math. Soc. 39 (1989), 301-317

[30] G.M. Kelly and A.J. Power: Adjunctions Whose Counits are Coequalizers, and Presentations of Finitary Enriched Monads, J. Pure Appl. Alg. 89 (1993), 163-179

[31] I. Kriz: On Spin and Modularity in Conformal Field Theory, Ann. Sci. École Norm. Sup.(4) 36 (2003), no.1, 57-112

[32] S. Lack: Codescent Objects and Coherence, J. Pure Appl. Alg. 175 (2002) 223-241

[33] S. Lack: On the Monadicity of Finitary Monads, J. Pure Appl. Alg. 140 (1999), 65-73

[34] F.W. Lawvere: Functorial Semantics of Algebraic Theories, Proc. Nat. Acad. Scie. U.S.A. 50 (1963) 869-87

[35] F.W. Lawvere: Some Algebraic Problems in the Context of Functorial Semantics of Algebraic Theories, Reports of the Midwest Category Seminar. II, pp. 41-61, Springer, Berlin 1968

[36] F.W. Lawvere: Ordinal Sums and Equational Doctrines, Seminar on Triples and Categorical Homology Theory, pp 141-155, Springer, Berlin 1969 
[37] J. MacDonald and A. Stone: Soft Adjunction between 2-categories, J. Pure Appl. Alg. 60 (1989), 155-203

[38] S. Mac Lane and Ieke Moerdijk: Sheaves in Geometry and Logic, Springer Verlag, 1992

[39] S. Mac Lane: Categories for the Working Mathematician, Graduate Texts in Mathematics 5, Springer Verlag, 1998

[40] I. Moerdijk: Introduction to the Language of Stacks and Gerbes, arXiv:math.AT/0212266 v1 19 Dec 2002

[41] A.J. Power: A General Coherence Result, J. Pure Appl. Algebra 57 (1989), 165-173

[42] A.J. Power: Coherence for Bicategories with Finite Bilimits I, Categories in Computer Science and Logic, Contemporary Mathematics 92 (1989), AMS 341-347

[43] A.J. Power: Enriched Lawvere Theories, Theory Appl. Categ. 6 (1999), 83-93

[44] C. Schubert, "Topologische Strukturen als laxe Algebren," http://www.math.uni-bremen.de/ dmb/Public/dipl_chr_schubert.pdf

[45] G. Segal: The Definition of Conformal Field Theory, preprint from the 1980's

[46] H. Simmons: The Glueing Construction and Lax Limits, Math. Structures Comput. Sci. 4 (1994), no. 4, 393-431

[47] R.H. Street: Two Constructions on Lax Functors, Cahiers Top. et Geom. Diff. 13, (1972), 217-264

[48] R. H. Street: The Formal Theory of Monads, J. Pure Appl. Algebra 2 (1972), 149-168

[49] R.H. Street: Limits Indexed by Category-valued 2-Functors, J. Pure Appl. Algebra 8 (1976), 149-181

[50] R.H. Street: Fibrations in Bicategories, Cahiers Top. et Geom. Diff. 21 (1980), 111-160

[51] R.H. Street: Correction to "Fibrations in Bicategories", Cahiers Top. et Geom. Diff. 28 (1987), 53-56

[52] A. Vistoli: Notes on Grothendieck Topologies, Fibered Categories, and Descent Theory, Advanced School in Basic Algebraic Geometry July 2003 at abdus salam ictp http://agenda.ictp.trieste.it/agenda/current/fullAgenda.php?email=0 kida=a0255

Department of Mathematics, University of Michigan, Ann Arbor, Mi, 48109

E-mail address: fioret@umich.edu 